\title{
Earth-affecting solar transients: a review of progresses in solar cycle 24
}

Jie Zhang ${ }^{1 *}$ (D), Manuela Temmer ${ }^{2}$, Nat Gopalswamy ${ }^{3}$, Olga Malandraki ${ }^{4}$, Nariaki V. Nitta $^{5}$, Spiros Patsourakos ${ }^{6}$, Fang Shen ${ }^{7}$, Bojan Vršnak ${ }^{8}$, Yuming Wang ${ }^{9}$, David Webb ${ }^{10}$, Mihir I. Desai ${ }^{11,12}$, Karin Dissauer ${ }^{2,13}$, Nina Dresing ${ }^{14,15}$, Mateja Dumbović ${ }^{8}$, Xueshang Feng ${ }^{7}$, Stephan G. Heinemann ${ }^{2,16}$, Monica Laurenza ${ }^{17}$, Noé Lugaz ${ }^{18}$ and Bin Zhuang ${ }^{18}$

\begin{abstract}
This review article summarizes the advancement in the studies of Earth-affecting solar transients in the last decade that encompasses most of solar cycle 24. It is a part of the effort of the International Study of Earth-affecting Solar Transients (ISEST) project, sponsored by the SCOSTEPNarSITI program (2014-2018). The Sun-Earth is an integrated physical system in which the space environment of the Earth sustains continuous influence from mass, magnetic field, and radiation energy output of the Sun in varying timescales from minutes to millennium. This article addresses short timescale events, from minutes to days that directly cause transient disturbances in the Earth's space environment and generate intense adverse effects on advanced technological systems of human society. Such transient events largely fall into the following four types: (1) solar flares, (2) coronal mass ejections (CMEs) including their interplanetary counterparts ICMES, (3) solar energetic particle (SEP) events, and (4) stream interaction regions (SIRs) including corotating interaction regions (CIRs). In the last decade, the unprecedented multi-viewpoint observations of the Sun from space, enabled by STEREO Ahead/Behind spacecraft in combination with a suite of observatories along the Sun-Earth lines, have provided much more accurate and global measurements of the size, speed, propagation direction, and morphology of CMEs in both 3D and over a large volume in the heliosphere. Many CMEs, fast ones, in particular, can be clearly characterized as a two-front (shock front plus ejecta front) and three-part (bright ejecta front, dark cavity, and bright core) structure. Drag-based kinematic models of CMEs are developed to interpret CME propagation in the heliosphere and are applied to predict their arrival times at $1 \mathrm{AU}$ in an efficient manner. Several advanced MHD models have been developed to simulate realistic CME events from the initiation on the Sun until their arrival at 1 AU. Much progress has been made on detailed kinematic and dynamic behaviors of CMEs, including non-radial motion, rotation and deformation of CMES, CME-CME interaction, and stealth CMEs and problematic ICMEs. The knowledge about SEPs has also been significantly improved. An outlook of how to address critical issues related to Earth-affecting solar transients concludes this article.
\end{abstract}

Keywords: Coronal mass ejection, Interplanetary coronal mass ejection, Solar energetic particle, Corotating interaction region, Flare, Corona, Sun, Geomagnetic storms, Space weather

\footnotetext{
* Correspondence: jzhang7@gmu.edu

${ }^{1}$ Department of Physics and Astronomy, George Mason University, 4400

University Dr., MSN 3F3, Fairfax, VA 22030, USA

Full list of author information is available at the end of the article
}

\section{Springer Open}

(c) The Author(s). 2021 Open Access This article is licensed under a Creative Commons Attribution 4.0 International License, which permits use, sharing, adaptation, distribution and reproduction in any medium or format, as long as you give appropriate credit to the original author(s) and the source, provide a link to the Creative Commons licence, and indicate if changes were made. The images or other third party material in this article are included in the article's Creative Commons licence, unless indicated otherwise in a credit line to the material. If material is not included in the article's Creative Commons licence and your intended use is not permitted by statutory regulation or exceeds the permitted use, you will need to obtain permission directly from the copyright holder. To view a copy of this licence, visit http://creativecommons.org/licenses/by/4.0/. 


\section{Introduction}

Earth-affecting solar transients refer to a broad range of energetic and/or eruptive events occurring on the Sun that have direct effects on the space environment near the Earth and cause adverse space weather impact on advanced technological systems of human society. They occur near the Sun on timescales of minutes to hours, and the resulting effects on the Earth can take place in minutes to days. These transient events are commonly categorized into four different types: (1) solar flares, (2) coronal mass ejections (CMEs) and their interplanetary counterparts, Interplanetary CMEs (ICMEs), (3) solar energetic particle (SEP) events, and (4) stream interaction regions (SIRS) including corotating interaction regions (CIRs). These four types of Earth-affecting transient events differ in their observational appearances, physical origin or processes, as well as the geoeffectiveness in their own unique ways (Table 1). Other energetic events on the Sun, such as filament eruptions, coronal dimmings, waves, etc., can be usually treated as an associated phenomenon with solar flares and/or CMEs. In the following, we briefly introduce the definition of these phenomena and their possible geoeffectiveness, along with selected review articles that discuss in depth these phenomena. The detailed review of these phenomena, including theoretical interpretations and numerical modelings, is given in the subsequent sections of this article.

\subsection{Solar flares}

Solar flares are probably the oldest transient phenomenon ever observed on the Sun. They were first discovered as a flash in white light by Carrington (1859) and Hodgson (1859) when observing sunspots. In the modern era, solar flares are observed as sudden enhancement in electromagnetic radiation over a broad range of wavelengths including radio, visible light, EUV, $\mathrm{X}$-rays, and gamma rays. The radiation energy released during a flare is about $10^{28}$ to $10^{32}$ ergs during a timescale of minutes to hours. It is well accepted that magnetic reconnection in a configuration of current sheet is the central mechanism that converts free magnetic energy in the corona into particle acceleration and plasma heating, producing a solar flare. Shibata and Magara (2011) provided a review on solar flares with a focus on the theoretical magnetohydrodynamic process. Fletcher et al. (2011) made a review on solar flares from an observational point of view. Hudson (2011) discussed flares from the perspective of their global properties. Note that it is now well known that the process of flares is strongly coupled with that of CMEs (Harrison 1995; Zhang et al. 2001; Temmer et al. 2008). Therefore, any further discussion on the origin of flares will be included in the discussions on the origin of CMEs, which will be extensively reviewed in this article. Very often, the term solar eruptions is used to refer to transient and largescale energy release on the Sun, and a solar eruption contains both flare and CME, along with other associated phenomena, such as coronal dimmings and global coronal waves, etc.

While the total electromagnetic radiation, or irradiance from the Sun, is nearly a constant with an amplitude of approximately $0.1 \%$ over the 11 -year solar cycle that affects the long-term climate of the Earth (Lean 1991), the EUV and X-ray irradiances during solar flares can increase by many folds to orders of magnitude. One space weather effect of solar flares is from EUV radiation in particular the Lyman-alpha radiation at $121.6 \mathrm{~nm}$ wavelength absorbed in the Earth's upper atmosphere causing its instantaneous heating and expansion, which results in a sudden drag and lowering of low-orbiting

Table 1 Four types of Earth-affecting solar transients and their key physical processes and geoeffectiveness

\begin{tabular}{|c|c|c|c|}
\hline $\begin{array}{l}\text { Earth- } \\
\text { affecting } \\
\text { solar } \\
\text { transients }\end{array}$ & Key physical processes & $\begin{array}{l}\text { Effects on near-earth space } \\
\text { environment }\end{array}$ & Effects on technological system and life \\
\hline Solar flares & $\begin{array}{l}\text { Magnetic reconnection; ideal MHD instability; } \\
\text { particle acceleration; plasma heating }\end{array}$ & $\begin{array}{l}\text { Disturbances in the ionosphere; } \\
\text { heating and expansion of upper } \\
\text { atmosphere }\end{array}$ & $\begin{array}{l}\text { High-frequency radio communication; satellite } \\
\text { drag (Earth climate from long-term variation } \\
\text { of solar irradiance) }\end{array}$ \\
\hline $\begin{array}{l}\text { CMEs and } \\
\text { ICMEs }\end{array}$ & $\begin{array}{l}\text { Ideal MHD instability; flux rope formation; shock } \\
\text { formation; particle acceleration; aerodynamic drag; } \\
\text { CME-CME interaction; magnetic reconnection }\end{array}$ & $\begin{array}{l}\text { Geomagnetic storms; substorms; } \\
\text { disturbances in the ionosphere; } \\
\text { ionosphere scintillations; radiation } \\
\text { belt storms }\end{array}$ & $\begin{array}{l}\text { GPS systems and navigation; satellite } \\
\text { communication; high-frequency radio com- } \\
\text { munication; electric power transmission; satel- } \\
\text { lite degradation and failure (single event } \\
\text { upset; dielectric material charging and dischar- } \\
\text { ging; surface charging); radiation hazards to } \\
\text { astronauts; radiation hazards to aircraft crew } \\
\text { and passengers }\end{array}$ \\
\hline SEPS & $\begin{array}{l}\text { Particle acceleration; injection; propagation; } \\
\text { turbulence }\end{array}$ & Particle radiation storms & $\begin{array}{l}\text { Satellite degradation and failure; high- } \\
\text { frequency radio communication; radiation } \\
\text { hazards to astronauts; atmospheric chemistry }\end{array}$ \\
\hline SIRs/CIRs & Stream interaction; particle acceleration & Substorms; geomagnetic storms & Similar to CMEs to a lesser extent \\
\hline
\end{tabular}


satellites (Schwenn 2006). Enhanced X-ray emissions from solar flares can penetrate to the bottom of the ionosphere and create an enhancement in the electron content, which may affect high-frequency radio communication.

\subsection{CMEs and ICMEs}

Since the discovery of solar flares by Carrington in 1859, it had long been conceived that there was a cause and effect relation between solar flares and geomagnetic activities on the Earth. Only starting from the 1980s, it became clear that the only type of solar transients that has a clear cause-effect relation to geomagnetic activity lies in CMEs, not in flares (e.g., Schwenn 1983; Sheeley Jr et al. 1985; Gosling 1993; Reames 1999; Zhang et al. 2007). It is now well accepted that CMEs are the solar transients that have the most profound effect on the space environment and inflict the most adverse space weather effect (Gopalswamy 2016).

CMEs are transient and energetic expulsion of mass and magnetic flux on a large scale from the low corona into interplanetary space. While the basic configuration of shock-driven magnetic structures from the Sun has been proposed to explain geomagnetic storm sudden commencement (Gold 1962) and various types of non-thermal solar radio bursts (Fokker 1963), CMEs were first directly imaged in white light from space by the OSO-7 coronagraph in the early 1970s (Tousey 1973). The speed of CMEs in the outer corona ranges from $\sim 100 \mathrm{~km} / \mathrm{s}$ to $\sim 3000 \mathrm{~km} / \mathrm{s}$ at $\max -$ imum with an average speed from 300 to $500 \mathrm{~km} / \mathrm{s}$ depending on the phase of the solar cycle (Yashiro et al. 2004). Mass of CMEs is mostly in the range from $10^{13}$ to $10^{16} \mathrm{~g}$ with a peak value at $3.4 \times 10^{14} \mathrm{~g}$, and their kinetic energy is mostly between $10^{27}$ to $10^{32}$ ergs with a peak value at $8.5 \times 10^{29}$ ergs (Vourlidas et al. 2010). CMEs reach their peak speed in the time range from minutes to hours with a median value at $\sim 54$ min (Zhang and Dere 2006).

The following is a list of review articles on CMEs, or more generally in solar eruptions, in the last decade. Schrijver (2009) reviewed the drivers of major solar flares and eruptions with a focus on flux emergence and its interaction with the ambient field. Chen (2011) provided a comprehensive overview of theoretical models and their observational basis of CMEs. The review of Webb and Howard (2012) focused the observational aspects of CMEs. Schmieder et al. (2015) further reviewed on observational perspectives of flareCME models. Gopalswamy et al. (2016) reviewed major discoveries on CMEs observed by spaceborne coronagraphs that show the growing significance of CMEs as the primary source of severe space weather. More recently, reviews were made focusing on magnetic structures of solar eruptions, from the perspective of magnetic flux rope (Cheng et al. 2017) and modeling of magnetic field (Guo et al. 2017). Chen (2017) made a review on the physics of erupting solar flux ropes in the aspects of both theory and observation. The origin, early evolution and predictability of solar eruptions were recently reviewed by Green et al. (2018).

The recent review of Patsourakos et al. (2020) discusses the formation and the nature of the pre-eruptive magnetic configuration of CMEs. We would also like to point out the following earlier reviews on CME models (Forbes 2000; Klimchuk 2001; Lin et al. 2003). Most recently, Lamy et al. (2019) made an extensive review on statistical properties of CMEs covering two complete solar cycles 23 and 24. Gopalswamy et al. (2020b) also reviewed how CME properties varied with the solar cycle, taking advantage of the availability of uniform and extensive observations made over two complete solar cycles.

Following the ejection from the corona, a CME largely maintains its magnetic configuration or topology that is well organized by a twisted magnetic flux rope, thus is able to continuously propagate outward through the heliosphere to a large distance, interacting with the ambient solar wind and impacting planets along its path. Its counterpart in the heliosphere is called interplanetary CME (ICME). Howard and Tappin (2009) reviewed the theory of ICMEs observed in the heliosphere. Rouillard (2011) provided a short review relating white light CMEs near the Sun and in situ ICMEs. Zhao and Dryer (2014) summarized the status of $\mathrm{CME} /$ shock arrival time prediction to that date. The physical processes of CME/ICME evolution are reviewed in Manchester et al. (2017). Lugaz et al.'s (2017) review focused on the interaction of successive CMEs. Shen et al. (2017) also reviewed on CME interaction with a focus on analyzing the physical nature of the interaction. More recently, Vourlidas et al. (2019) made an overview of predicting the geoeffectiveness properties of CMEs, including current status, open issues, and a path forward. The review by Kilpua et al. (2019) focused on the forecasting of magnetic structure and orientation of CMEs. The most recent review on ICMEs was by Luhmann et al. (2020).

Besides the ejecta or magnetic flux rope component, a fast CME is capable of driving a wider shock ahead and forming a thick sheath region between the eject front and the shock front. While the shock is the main source of solar energetic particles (SEPs), the sheath, like the magnetic flux rope, is also an important transient structure for causing geomagnetic storms. The properties and importance of shock, sheath regions, as well as CME ejecta, are viewed in (Kilpua et al. 2017; 2017a). 
ICMEs passing the Earth can significantly distort and energize the Earth's magnetosphere and generate a cascade of effects in the different layers of the Earth's space environment, including in the magnetosphere, radiation belt, ionosphere and upper atmosphere, and even in the lithosphere. These are collectively defined as space weather. The term space weather refers to conditions on the Sun and in the solar wind, magnetosphere, ionosphere, and thermosphere that can influence the performance and reliability of space-borne and ground-based technological systems and that can affect human life and health (Schwenn 2006). The terrestrial perspective and impacts of space weather are summarized in Pulkkinen (2007). Space weather effects in the Earth's radiation belts were recently reviewed in Baker et al. (2018). CMEs can cause extensive ionospheric anomalies (e.g., Wang et al. 2016a, b, c, d), and disturbances in the atmosphere-ionosphere coupling system (Yiğit et al. 2016). The cradle to grave process of some extreme space weather events is outlined in Riley et al. (2018a). Bocchialini et al. (2018) studied a large number of storm sudden commencement (SSCs), traced their origin to solar sources, identified their effects in the interplanetary medium, and investigated the corresponding response of the terrestrial ionized and neutral environment.

The impacts due to severe space weather storms caused by CMEs/ICMEs on technological systems are profound (Lanzerotti 2017). These impacts include hazards to astronauts, satellite degradation, and failure through single event upset. dielectric material charging and discharging and surface charging, error and failure in GPS navigation, effects on satellite communication, effects on high-frequency communication, effects on power grids and aviation, etc. The potential catastrophic societal effect of the May 1967 great storm is revisited by Knipp et al. (2016). The economic impact of space weather is reviewed and analyzed in Eastwood et al. (2017).

\subsection{SEPs}

Solar energetic particle events are enhancements of electrons, protons and heavy ion fluxes observed in the heliosphere related to both solar flares and CMEs. SEP events present energy spectra that span more than six orders of magnitude, from a few $\mathrm{keV}$ superthermal to $\mathrm{GeV}$ relativistic energies.

High energy particles from the Sun were first observed as a sudden increase in intensity in ground-based ion chambers and neutron monitors during large solar flares (Forbush 1946). Such ground-level enhancements (GLEs) consist of the strongest set of SEPs events that are mostly detected from space. For half a century following its discovery, it was generally assumed that energetic particles originated from solar flares, i.e., the point-like source in time and space. However, in the 1990s, it became clear that there are two types of SEPs: impulsive type and gradual type, whose source is of impulsive flares on the Sun and of large-scale long-lasting shocks driven by CMEs, respectively (Reames 1999). The gradual SEPs typically last for several days, while the impulsive events only last for a few hours. It is now believed that CME-driven coronal and interplanetary shocks are the most prolific producers of SEPs that pose radiation hazards for our environment and our assets on Earth and in space. Particle enhancements accompanying CME-driven interplanetary shocks that are passing near the Earth are known as energetic storm particles or ESP events, because they are often associated with "Sudden Storm Commencements."

Besides the seminal review paper that summarized the paradigm shift on the origin of SEPs in (Reames 1999), a series of review papers were also published in the last decade (Reames 2013, 2015, 2018, 2020). Reames (2013) provided a comprehensive account on the two sources of solar energetic particles, which highlighted the early evidence from fast-drifting type III and slow-drifting type II solar radio emissions (Wild et al. 1963). Reames (2015) focused on element abundances and source plasma temperatures of SEPs. Reames (2018) extended the topics including abundances, ionization states, temperatures and FIP (First Ionization Potential) in SEPs. Most recently, Reames (2020) categorized SEPs into four basic populations and discussed the four distinct pathways, to account for the mixture of SEPs from pure impulsive and pure gradual events. Desai and Giacalone (2016) provided a comprehensive review on large gradual SEP events to date. Klein and Dalla (2017) also made a review on the acceleration and propagation of SEPs. More recently, a set of 10 review papers on SEPs are collected in a published book (Malandraki and Crosby 2018b), which built upon the 2-year HESPERIA (High Energy Solar Particle Events Forecasting and Analysis) project of the EU HORIZON 2020 program.

Like flares and CMEs, it is apparent that SEPs pose a threat to modern technology strongly relying on spacecraft and are a serious radiation hazard to humans in space (Jiggens et al. 2014; Malandraki and Crosby 2018a). High energy charged particles have been found to have damaging impacts on various components of spacecraft, including instruments, electronic components, solar arrays etc. SEPs also effect signal propagation between Earth and satellites due to Polar Cap Absorption (PCA) which results from intense ionization of the D-layer of the polar ionosphere. In the instances when SEP events reach aviation latitudes, they are also a concern for human health as the radiation dose received can increase. This applies specifically to high latitude flights and polar routes for commercial aviation. It can be a risk for frequent flyers and for aircrew. SEP 
forecasting is relied upon to mitigate against the effects SEP events.

\section{$1.4 \mathrm{SIRs} / \mathrm{CIRs}$}

The solar wind reveals long-term, and most often periodic, variations in terms of high speed solar wind streams that may lead to geomagnetic storms. The solar wind also transports short-term disturbances, such as CMEs. Investigating the solar wind is therefore of crucial interest for Space Weather forecasting. The interplay between fast and slow solar wind causes stream interaction regions (SIRs). SIRs are related to coronal holes $(\mathrm{CHs})$, long-lived regions on the Sun with predominantly open magnetic field. Due to the quasi-stationary location of low-latitude $\mathrm{CHs}$, the interaction of high and slow speed solar wind streams results in a compression of plasma and magnetic field that occurs at certain distance from the Sun. As the Sun rotates, recurring SIRs are referred to as corotating interaction regions (CIRs).

Recent reviews on solar wind high speed streams are given by Cranmer, Gibson, and Riley et al. (2017); Living review by Richardson (2018) on SIRs and corona and solar wind by Cranmer and Winebarger (2019). Cranmer et al. (2017) gives an overview of the community's recent progress and understanding of two major problems associated with HSSs: the coronal heating and the acceleration of the fast and slow solar wind. They discuss recent observational, theoretical, model and forecasting techniques with a positive forecast to the future. The review by Richardson (2018) focuses entirely on the interaction of slow and fast solar wind leading to the formation of SIRs and discusses the acceleration processes of energetic particles in stream-stream interaction regions, modulation of the galactic cosmic ray count, resulting geomagnetic disturbances as well as MHD modeling results. The very recent review by Cranmer and Winebarger (2019) gives detailed insight into highresolution observations of the solar corona as well as 3D numerical simulations which hint towards small-scale entangled, twisted, and braided magnetic fields. These processes, which may lead to reconnection, are, despite their limitations, thought to be a main source of heating the solar corona.

The geoeffectiveness and Space Weather impact of SIRs/CIRs can be observed as variations in the Earth's magnetosphere, ionosphere, and even neutral density in the thermosphere. The solar wind delivers significant energy that causes various Space Weather phenomena. CIR-related storms are more hazardous to space-based assets, particularly at geosynchronous orbit compared to CMEs, because CIRs are of longer duration and have hotter plasma sheets causing a stronger spacecraft charging (Borovsky and Denton
2006). Details on the geoeffectiveness of SIRs/CIRs can be found in recent reviews and statistical papers (Kilpua et al. 2017; Vršnak et al. 2017; Yermolaev et al. 2018).

\subsection{ISEST project}

This review article is part of the collective effort made by the International Study of Earth-affecting Solar Transients (ISEST) project, which is one of the four research projects of the Variability of the Sun and Its Terrestrial Impact (VarSITI)) program, sponsored by the Scientific Committee on Solar-Terrestrial Physics (SCOSTEP) for the period of 2014 - 2018. The VarSITI program is summarized in a companion article (Shiokawa and Katya 2020 to be added). The stated overarching goal of the ISEST project is to understand the origin, propagation, and evolution of solar transients through the space between the Sun and the Earth, and develop the prediction capability of space weather. Toward this goal, the ISEST project has organized four dedicated workshops in three different geographic locations across the globe: $17-20$ June 2013 in Hvar, Croatia, 26 - 30 October 2015 in Mexico City, Mexico, 18 - 22 September 2017 in Jeju, South Korea, and 24 - 28 September 2018 again in Hvar, Croatia. The ISEST project maintains a standing website for hosting event catalogs, data, and presentations, and offers a forum for discussion at http://solar. gmu.edu/heliophysics/index.php/Main_Page.

The ISEST project has resulted in a Topic Issue in the journal of Solar Physics with a collection of 32 articles (Zhang et al. 2018a); this collection is then converted to a published book (Zhang et al. 2018b). A similar but earlier project in the CAWSES-II era, Climate and Weather of the Sun-Earth System of SCOSTEP (2010-2014), is the project of "Short-term variability of the Sun-Earth system"; the summary of the activity from 2010-2014 is in Gopalswamy et al. (2015c).

The implementation of the ISEST project is centered around several working groups, which are (1) data, (2) theory, (3) simulation, (4) campaign study, (5) SEP, (6) Bs challenge, and (7) MiniMax24 campaign. The sections of this article largely contain the contribution of these seven working groups, respectively. We organize the articles as follows. Section 2 is on observational progress on CMEs and ICMEs. Section 3 is on theoretical progress on CMEs and ICMEs, while Section 4 summarizes the progress in simulation studies of CMEs and ICMEs. Campaign-style studies are reviewed in Section 5. SEP studies are reviewed in Section 6. Section 7 reviews stream interaction regions. Forecasting CMEs are reviewed in Section 8. Section 9 summarizes the activity of MiniMax24 campaign. The conclusion and outlook are in Section 10. 


\section{Progress in observations of CMEs/ICMEs 2.1 Introduction}

The capacity of observing and studying the solarterrestrial system has increased dramatically during solar cycle 24. We can observe and track solar eruptions nearly continuously in time and space from Sun to Earth, thanks to a large set of sensitive remote-sensing and in situ instruments onboard a fleet of spacecraft. These include the Solar Terrestrial Relations Observatory Ahead/Behind (STEREO A/B) (twin spacecraft launched in 2006; drifting along the Earth orbit) (Kaiser et al. 2008), the Solar Dynamics Observatory (SDO) (geosynchronous orbit; launched in 2010) (Pesnell et al. 2012), the Solar and Heliospheric Observatory (SOHO) (L1 point; launched in 1995) (Domingo et al. 1995), Hinode mission (polar orbit of Earth; launched in 2006) (Kosugi et al. 2007), the Advance Composition Explorer (ACE) (L1 point; launched in 1997) (Smith et al. 1998), Wind spacecraft (L1 point; launched in 1994) (Ogilvie et al. 1995), and other space-based spacecraft and groundbased observatories.

In particular, the SECCHI (Sun Earth Connection Coronal and Heliospheric Investigation) suite onboard STER EO comprised five imaging telescopes, which together observe the solar corona from the solar disk to beyond 1 AU; these telescopes are EUVI (Extreme Ultraviolet Imager: 1-1.7 Rs), COR-1 (Coronagraph 1: 1.5-4 Rs), COR-2 (Coronagraph 2: 2.5-15 Rs), HI-1 (Heliospheric Imager 1: $15-84 \mathrm{Rs}$, or $4-24^{\circ}$ in elongation angle) and HI-2 (Heliospheric Imager 2: 66-318 Rs, or $19-89^{\circ}$ in elongation angle ) (Howard et al. 2008). Two recent missions dedicated to solar and heliospheric physics are the Parker Solar Probe (PSP) (varying elliptical orbit around the Sun with perihelia < 10 Rs; launched in 2018) (Fox et al. 2016) and the Solar Orbiter (highly elliptical and inclined orbit around the Sun; launched in 2020) (Müller et al. 2020). These missions will provide observations of the unexplored territories of the Sun-heliosphere system, but results from these two spacecraft will not be included in this review.

The global and long-lasting nature of CMEs makes the so-called Sun-Earth connection truly meaningful. Erupting from the low corona of the Sun and propagating into the outer corona and interplanetary space, a typical fast CME largely contains two volumetric components that are persistent in time and space: the magnetic ejecta component and the shock sheath component. Each of the two volumetric components has its own unique front: the ejecta front and the shock front, respectively. This is evident in both remote-sensing imaging observations in white light as well as from in situ one-point time-series sampling when the CME passes through the spacecraft, as illustrated in Fig. 1. The ejecta contains the erupted magnetic field and plasma originating in the low corona, while the sheath region contains the magnetic field and plasma corresponding to the ambient solar wind that is disturbed and compressed by the forward shock (or forward compressing waves for slower CMEs). Through its propagation from the Sun to Earth, a CME ejecta is believed to maintain its curved flux-rope shape in a quasi-self-similar manner and keep its two legs remaining rooted on the surface of the Sun for days and even longer.

In light of the fact that the behavior of CMEs is dominated by different kinematics and dynamics at different distances within the vast space between the Sun and Earth, we loosely divide the whole Sun-Earth domain into three sub-domains: (1) in the corona where CME evolution is dominated by its internal magnetic force; this is also the region imaged by coronagraphic instruments, (2) farther from the Sun in the interplanetary space where CME evolution is mostly dominated by the aerodynamic drag force, i.e., momentum transfer between the CME and the ambient solar wind flow; practically, this is the area observed by Heliospheric Imagers onboard STEREO, and (3) near the Earth (or other locations near $1 \mathrm{AU}$ ) where most in situ sampling data are taken. These in situ data provide detailed diagnostics of plasma, magnetic, and abundance properties of $\mathrm{CME}$ ejecta and driven shock, albeit limited at one particular point in space or a particular sampling line for a traveling ICME. Remote-sensing of the CME-driven shock has been enabled by tracking type II radio bursts with the radio instruments on board the Wind and STEREO missions. A CME in these three sub-domains can be conveniently called as CME in the traditional sense, an ICME in the interplanetary space and in situ ICME, respectively. Note that there is certainly no boundary or barrier between the aforementioned corona and interplanetary space, which can be anywhere between 4 Rs and 30 Rs. For the sake of simplicity only, one could arbitrarily adopt a value of 20 Rs (roughly coinciding with the Alfvenic critical point) to separate domains 1 and 2.

In this Section, we review the basic morphology and geometry (Section 2.2) as well as kinematic behavior of CMEs (Section 2.3) in the corona and in the interplanetary space. The properties of source regions in the low corona where CMEs originate are reviewed in Section 2.4. Section 2.5 reviews statistical properties and solar cycle variation of CMEs and ICMEs. A summary is given in Section 2.6

\subsection{CME morphology, geometry and their evolution 2.2.1 Basic morphology of CMEs}

One of the fundamental properties of CMEs is its morphology near the Sun, as obtained from the direct interpretation of outer corona images made by whitelight coronagraphs. Prior to the $\mathrm{SOHO}$ era, the 


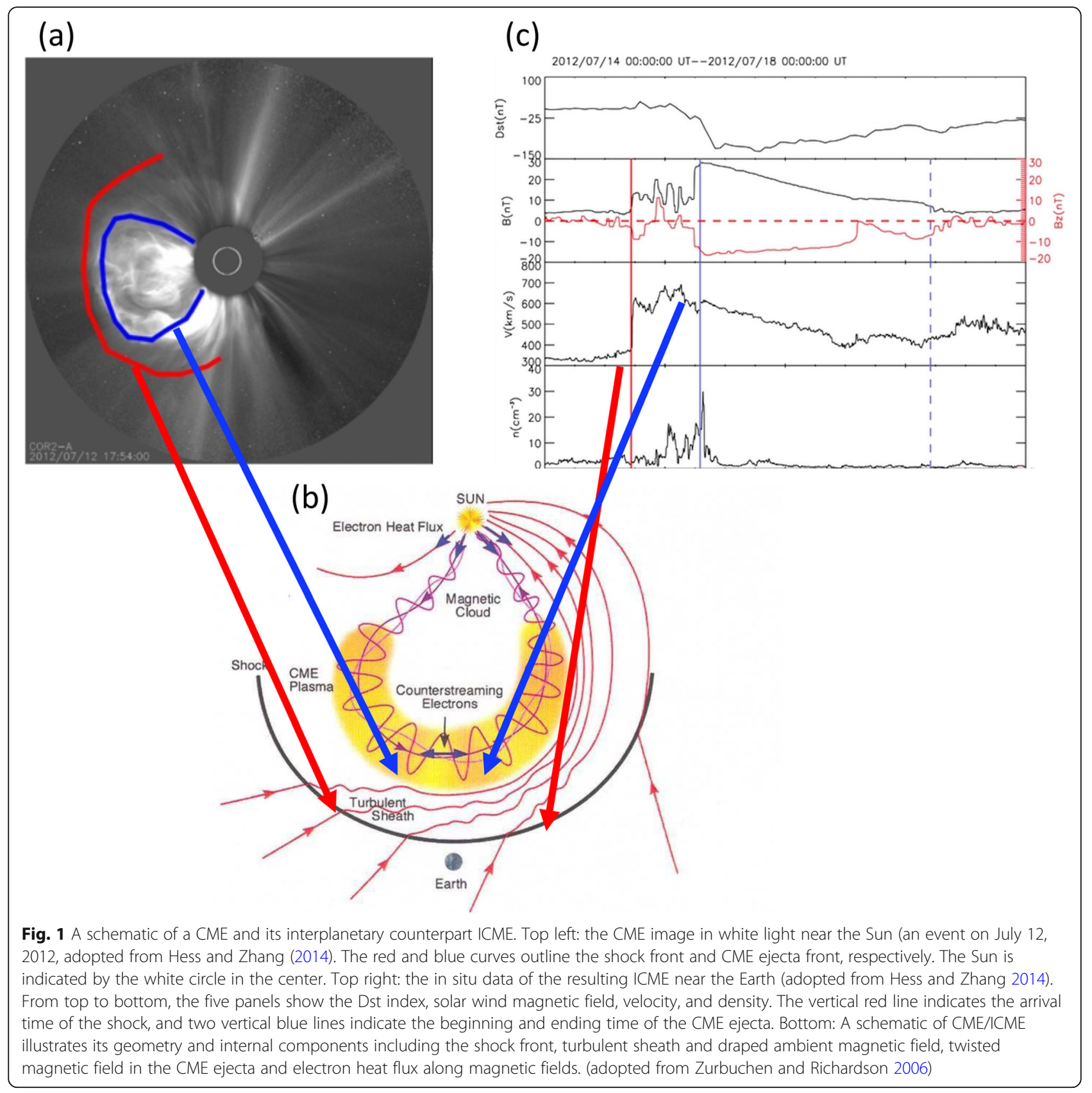

morphology of CMEs had been characterized by the so-called three-part structure: a bright frontal shell, followed by a relatively dark cavity surrounding a bright core (Illing and Hundhausen 1986). The expected shock fronts missing in this traditional structure were later routinely identified, thanks to the improved sensitivity of coronagraphs on the SOHO and STEREO spacecraft. The shock front appears as an outline or boundary of a weakly brightened region that contains displaced or kinked coronal streamers and rays (Sheeley et al. 2000; Wood and Howard 2009; Ontiveros and Vourlidas 2009; Hess and Zhang
2014; Liu et al. 2017a; Gopalswamy et al. 2009a, 2009b; Gopalswamy and Yashiro 2011). A shock fronts is expected to form when the speed of the CME ejecta in the frame of the ambient solar wind is faster than the fast mode wave speed. Figure 2 shows one example of the identification and geometrical fitting of the fronts of the ejecta (green wireframe) and the shock (red wireframe). Thus, the overall morphology of a typically large and fast CME can be characterized by two fronts: a large fuzzy shock/wave front followed by a bright loop-like ejecta front which can be interpreted as the plasma pileup at the boundary of 


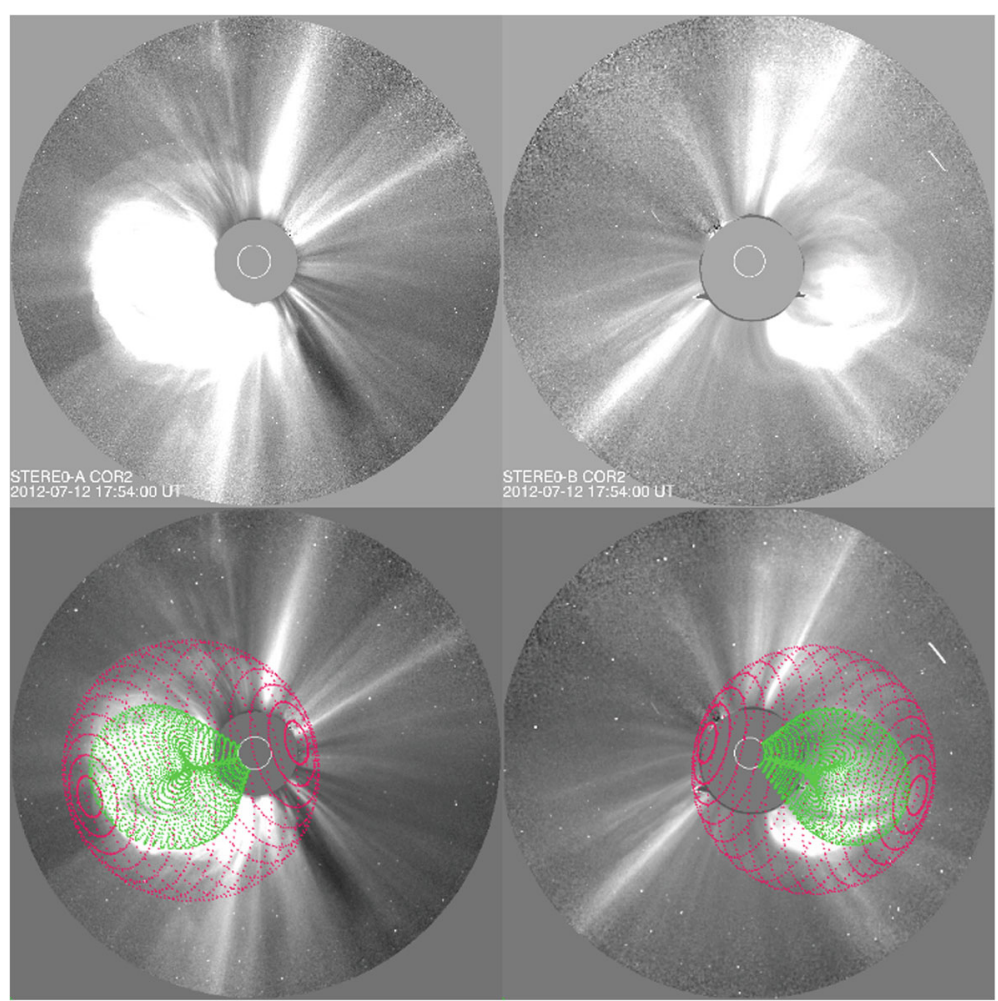

Fig. 2 Forward model fitting of CME ejecta front and CME-driven shock front of July 12, 2012 event based on STEREO-A COR2 (left) and STEREOB COR (right) images, along with (bottom) and without the raytrace mesh. The green mesh shows the GCS fitting to the eject front, whereas the red mesh shows the spheroid fitting to the shock front (adapted from Hess and Zhang 2014)

the expanding magnetic flux rope, irrespective of whether a three-part structure can be identified following the looplike front (Vourlidas et al. 2013).

To quantitatively capture the 3D morphology (i.e., shape and size) and geometry (i.e., location and orientation) of a CME ejecta, the graduated cylindrical shell (GCS) model has been widely used (Thernisien et al. 2006; Thernisien et al. 2009; Thernisien 2011). This 3D geometric model, meant to reproduce fluxrope-like CMEs, consists of a tubular section forming the main body of the flux rope attached to two cones that correspond to the "legs" of the flux rope that are connected to the surface of the Sun. In this model, the bright frontal shell of the ejecta corresponds to the surface of the flux rope, and the cavity as the body of the flux rope, being consonant with the common view (e.g., Chen et al. 1997a; Cremades et al. 2006). This model contains a central axis that threads through the center of the curved tube and the conical legs. The shell surface of the flux rope exhibits rotational symmetry around the central axis at each cross section perpendicular to the axis. This axis also defines the geometric plane of the flux rope. The GCS model, as the one implemented in Solar Software
(IDL), has the following six free parameters, (1) propagation longitude, (2) propagation latitude, (3) tilt angle of the curved central axis (the plane of the flux rope), (4) height of the leading edge of the front, (5) half angle between the axes of the legs and (6) aspect ratio between the radius of the circular cross section of the tubular shell and the distance to the outer edge of the shell from the Sun center; for details of the geometry and the parameters, refer to Fig. 1 in Thernisien et al. (2009). The first three parameters define the geometry, while the later three parameters define the morphology or sizes of the CME. As will be discussed below, CME geometry changes significantly close to the Sun, but is assumed to remains largely constant in the interplanetary space. On the contrary, CME morphology remains self-similar in the corona, but distorts significantly in the interplanetary space.

To capture both the ejecta and shock fronts of CMEs, Kwon et al. (2014) developed a compound model, in which the shock front is modeled as an ellipsoid, which can be spherical or ellipsoidal depending on events as well as on the evolution stage of the event of study; the ejecta front simply follows the GCS model. The ellipsoid model of the shock front also has six free 
parameters that define the geometry and morphology in 3D. Using this model, Kwon et al. (2014) demonstrated that the footprints of expanding shock waves seen in the outer corona correspond well to the EUV wave front observed on the solar disk in the early development of CMEs. Similar results of reconciling CME-driven shock and EUV coronal waves are obtained in other studies (Cheng et al. 2012; Veronig et al. 2018), revealing the global behavior of CMEdriven shocks.

The reconstruction of shock fronts in 3D also reveals the global properties of halo CMEs. It had been widely believed that the halo appearance of a CME is caused by the geometric projection effect, i.e., a CME moves along the Sun-Earth line and project in all directions on the plane of the sky surrounding the occulter. However, Kwon et al. (2015) found that $66 \%$ of halo CMEs from 2010 to 2012 are seen as halos in all three spacecraft, SOHO, STEREO-A and STREO-B when they are in quadrature configuration. They concluded that the halo structure largely represents the shock/wave that propagates in all directions, with a lesser dependence on the projection effect of the CME ejecta that has a limited size. Shen et al. (2013a, b, c) also found that very fast $(>900 \mathrm{~km} / \mathrm{s})$ full-halo CMEs originating far from the vicinity of solar disk center have a small projection effect. This global reach of CME-driven shock, even having a component propagating in the opposite direction of the CME ejecta, helps explain that some SEP events have a wide range of helio-longitude distribution, even allowing particle intensity increase at poorly connected spacecraft (Lario et al. 2014; Liu et al. 2017a).

While the physical properties of CME-driven shocks were refined in the last decade, as discussed above, the physical nature of the core of CMEs has been recently questioned in several studies (Howard et al. 2017; Song et al. 2017; Veronig et al. 2018; Song et al. 2019). It has long been believed that the bright core inside the CME cavity originates from entrained erupting filament/prominence material which has a high density. However, through investigating source region of CMEs on the solar disk and tracking eruptions continuously into the coronagraph FOV from multiple viewpoints in space, unambiguous observational evidence shows that many "classical" threepart CMEs do not contain an erupting filament/prominence (Howard et al. 2017; Song et al. 2017). Howard et al. (2017) suggested that the core could be the result of a mathematical caustic produced by the geometric projection of a twisted/writhed flux rope, implying the same flux rope produces both the cavity and core; they also suggested another possible cause that could arise spontaneously from the eruption of a flux rope. Through investigating the well-observed highly structured CME on
2017 September 10, Veronig et al. (2018) argued that the bright core rises from the hot plasma generated through magnetic reconnection but adds onto the rim of the rising flux rope, implying that the core is the flux rope.

Song et al. (2019) suggested that the core might correspond to the entirety of the flux rope in the early phase, but expand continuously and fill-in the entire cavity at a later time. The physical nature of the observed CME core and cavity remains to be an open question.

\subsubsection{CME morphological evolution in the corona: self- similar expansion}

How does the morphology of CMEs evolve in the corona (as well as in the interplanetary space; see next subsection)? This issue is far from settled. One simple question is whether such evolution is self-similar or not. Since it is a structured $3 \mathrm{D}$ entity, a CME evolves along three principal directions in a 3D space. Thus, to properly answer this question, one has to define which direction the self-similarity refers to. For the clarity of discussion hereafter, we define three principal directions in the frame of the flux rope with the apex of the central axis of the flux rope at the origin: toroidal direction $(\mathrm{T})$, poloidal direction $(\mathrm{P})$ and radial direction $(\mathrm{R})$. The radial direction is the vector line connecting the Sun center toward the apex of the central axis, the toroidal direction, which is on the plane of the flux rope, is along the direction of the central axis at the apex, while the poloidal direction is perpendicular to the plane of the flux rope. Both toroidal and poloidal directions are perpendicular to the radial direction. If the tilt angle of the flux rope is zero, the toroidal direction will be exactly along the heliographic longitude, while the poloidal direction will be along the heliographic latitude. The linear sizes of the flux ropes can be characterized by $L_{T}, L_{P}$ and $L_{R}$, respectively. Similarly, one can define three aspect ratios: $\kappa_{\mathrm{T}}=\mathrm{L}_{\mathrm{T}} / \mathrm{d}_{\mathrm{A}}, \kappa_{\mathrm{P}}=\mathrm{L}_{\mathrm{P}} / \mathrm{d}_{\mathrm{A}}, \kappa_{\mathrm{R}}=\mathrm{L}_{\mathrm{R}} / \mathrm{d}_{\mathrm{A}}$, respectively, where $\mathrm{d}_{\mathrm{A}}$ is the distance of the apex of the flux rope central axis. A constant aspect ratio along one particular direction defines the self-similar evolution in that direction.

Since the advent of multi-viewpoint observations of CMEs, the aforementioned GCS model is widely used to determine CME morphology in 3D for a large number of CMEs (e.g., Poomvises et al. 2010; Kilpua et al. 2012; Colaninno et al. 2013; Subramanian et al. 2014; Hess and Zhang 2014; Veronig et al. 2018; Chi et al. 2018b). These studies found good agreement between GCSgenerated flux rope shells and the observed CME appearances. One particular interesting result, relevant to the morphology, is that the constant aspect ratio and angular width can be adopted for a particular CME observed in different times, implying a self-similar evolution of the morphology in all three principle directions. 
Note that the aspect ratio in the GCS model is the same as $\kappa_{P}$ and $\kappa_{R}$ defined above, and $\kappa_{P}=\kappa_{R}$ since the GCS flux rope has a circular cross section perpendicular to the central axis. The angular width in the GCS model is equivalent to $\kappa_{\mathrm{T}}$ defined above. In other words, CME angular widths along both toroidal and poloidal directions remain constant as it evolves, and in the meantime, the CME expands radially at the same rate as along the two lateral directions, maintaining a circular cross section, or constant $\kappa_{R}$. However, the constant aspect ratio along the radial direction will not be true in the interplanetary space as discussed in the next subsection.

A more robust examination of self-similarity can be carried out by comparing expansion speed and bulk speed of CMEs, and a constant ratio with time indicates the self-similarity. Through a statistical study of 475 CMEs from 2007 -2014 that are geometrically well structured and whose geometric centroid and boundary can be well determined from single-viewpoint images (Vourlidas et al. 2017; Balmaceda et al. 2018), Balmaceda et al. (2020) found that (1) the relationship between lateral expansion and radial expansion speeds is linear and does not change with height, and (2) the ratio of the bulk propagation speed to the lateral expansion speed is a function of the angular width that follows the description of self-similar evolution. They also found that most CMEs achieve a self-similar evolution above 4 Rs, which is especially applicable to impulsively accelerated events.

However, in the inner corona (e.g., < 4 Rs), CMEs would not evolve in a self-similar manner and experience the so-called over-expansion, i.e., the aspect ratio increases with height and consequently the angular width also increases with height (Patsourakos et al. 2010; Balmaceda et al. 2020; Cremades et al. 2020). Studying a sizeable number of CMEs that could be tracked from their inception in the EUV low corona to the outer corona from multiple viewpoints, Cremades et al. (2020) found that CME angular widths, along both toroidal and poloidal directions, increase considerably with height below $\sim 3$ Rs, and the growth rate along the toroidal direction is higher than that along the poloidal direction. They also found that the ratio of the two expansion speeds is nearly constant after $\sim 4$ Rs, implying that CMEs there reach a state of self-similar expansion.

\subsubsection{CME geometric change in the corona: deflection and rotation}

On the top of morphological expansion of CMEs discussed above, the geometry of CMEs evolves in a manner that deviates from the simplest behavior of straight radial motion near the Sun: (1) deflection or non-radial motion, (2) rotation resulting in the change of the tilt angle of the CME. Both deviations pose a challenge for predicting hits or misses for Earth-directed CMEs and eventually whether a given CME would be geoeffective (Kay et al. 2017).

CME deflection has long been noticed (MacQueen et al. 1986; Gopalswamy et al. 2003; Cremades and Bothmer 2004). The deflection found in these observations was restricted along the latitude only, since they were made from single viewpoint observations from spacecraft along the Sun-Earth line. The deflection has a tendency that changes the direction of motion of CMEs from high latitude toward the low latitude equator during the solar minimum. This tendency implies that the deflection during solar minimum is related to the large-scale magnetic field from polar coronal holes (Gopalswamy et al. 2003; Cremades and Bothmer 2004). However, during the solar maximum, the directions of deflection can be complex, i.e., toward both higher and lower latitudes from the original position angle of CMEs.

Multiple viewpoint observations from STEREO provide much improved diagnostics of CME deflections, including the time evolution of deflections along both latitudinal and longitudinal directions. (Gopalswamy et al. 2003) found that a slow CME during solar minimum was deflected toward a lower latitude region by $\sim 30^{\circ}$, and demonstrated that such a deflection is caused by a non-uniform distribution of the background magnetic field, and the CME tended to propagate to the region with lower magnetic-energy density. A follow-up study on a larger sample of events further confirmed that the background magnetic field quantitatively described by the magnetic energy density control the deflection of CMEs along both longitude and latitude (Gui et al. 2011). Kilpua et al. (2009) showed that a CME originating in a high latitude crown prominence was guided by polar coronal hole fields to the equator and produced a clear ICME in the near-ecliptic solar wind at in situ. Such a scenario of large latitude deflection (e.g., $>30^{\circ}$ ) of a high-latitude CME moving toward the equator and intercepting the Earth was also reported in Byrne et al. (2010). Besides being influenced by coronal holes, Liewer et al. (2015) attributed the rapid initial asymmetric expansion or deflection of some CMEs in the inner corona $(<1.5 \mathrm{Rs}$ in EUVI FOV) to the magnetic pressure of active regions fields in the immediate vicinity of the eruption.

CME deflections in longitude were also recognized and studied, but to a lesser extent than in latitude. One of the earlier clues came from the fact that there was an east-west asymmetry of solar source regions of geoeffective CMEs, i.e., more geoeffective CMEs originated from 
the western hemisphere than from the eastern hemisphere (Zhang et al. 2003; Wang et al. 2004), thus favoring an interpretation of longitudinal deflection (Wang et al. 2004). Another line of evidence is related to the finding of "driverless" shocks found at 1 AU whose solar sources were near the solar disk center, indicating that the CME ejecta were deflected away from the Sun-Earth line (Gopalswamy et al. 2009b).

Direct measurements of longitudinal deflection only became possible with the advent of STEREO (Isavnin et al. 2013, 2014; Möstl et al. 2015; Mays et al. 2015). Based on a sample of 14 events, Isavnin et al. (2014) showed that most longitudinal and latitudinal deflections happened within $30 \mathrm{Rs}$, and a large part of the latitudinal deflection occurred within a few Rs. Möstl et al. (2015) studied a particularly interesting case of the January 7, 2014, CME, which originated near the disk center but was deflected toward the west by $\sim 37^{\circ}$ in longitude. Thus, this major CME (projected speed of $\sim 2400 \mathrm{~km} / \mathrm{s}$ and associated with an X1.2 flare) almost entirely missed the Earth and causing a false alarm of prediction by various space weather prediction centers. They also found that such a large longitudinal direction was attained very close to the Sun $(<2.1 \mathrm{Rs})$, likely caused in this particular case by the channeling of nearby active region magnetic fields rather than coronal holes. Such a surprising geomagnetic non-event from a major disk center eruption highlights the importance of knowing the true directionality of CMEs for space weather prediction (Mays et al. 2015).

Another very relevant geometric evolution of CMEs is the rotation, or the change of the tilt angle of the entrained magnetic flux rope. The tilt angle is zero if the toroidal axis of CMEs lies on the equatorial plane of the Sun, and $90^{\circ}$ if perpendicular to the equatorial plane. The tilt angle is critically important in deciding how much of the southward magnetic field will encounter the Earth for a given impacting CME, thus determining its expected intensity of geoeffectiveness (e.g., Bothmer and Schwenn 1998). Prior to the STEREO era, the evidence of rotation resided on the observations of erupting filaments in EUV coronal images (Ji et al. 2003; Zhou et al. 2006; Green et al. 2007). Using the orientation of the elongation of halo CMEs from single viewpoint LASCO observations as a proxy and assuming that the orientation of the post-eruption arcade in the source region is the CME orientation at the beginning of the eruption, Yurchyshyn et al. (2009) found that most CMEs appeared to rotate by $10^{\circ}$, but up to $30-50^{\circ}$ in some events.

Multiple viewpoint STEREO observations provide direct measurements of CME rotations in the coronagraphic field of view (Vourlidas et al. 2011; Isavnin et al.
2013, 2014; Liu et al. 2018d; Chen et al. 2019). Using the GCS model to define and track the 3D geometry of a slow CME on 2010 June 16, Vourlidas et al. (2011) found that the CME had an initial tilt of about $30^{\circ}$ at $2-$ 3 Rs, very similar to the orientation of the neutral line on the surface source, but later rotated by about $60^{\circ}$ when the CME traveled from 2 to 15 Rs. Liu et al. (2018d) studied a CME on 2015 December 16 and found that the tilt from the GCS model rotated by almost $95^{\circ}$ compared with the orientation on the source region; the same CME was also deflected by $45^{\circ}$ in longitude and $35^{\circ}$ in latitude. Such an extremely large rotation of the main structural axis was also found in an erupting filament based on STEREO observations in Song et al. (2018), who reported a counter-clockwise rotation of about $135^{\circ}$ of the filament in $\sim 26 \mathrm{~min}$ and then reversed to the clockwise rotation of $45^{\circ}$ in about $15 \mathrm{~min}$. Based on a statistical study of geometry of CMEs, Isavnin et al. (2014) noted that the rotation largely occurred below 5 Rs, but continued in the outer corona and the interplanetary space.

\subsubsection{Geometry of ICMEs in the interplanetary space}

As discussed above, in the inner corona $(\sim<4 \mathrm{Rs})$, a CME usually undergoes a super-expansion or non-selfsimilar increase of sizes in comparison with its distance from the Sun, and also experiences most geometric changes as defined by radial deflection and rotation of tilt angles. In the outer corona (e.g., from $\sim 4$ Rs to 20 Rs), on the other hand, a CME usually undergoes a selfsimilar expansion in all three principle directions and some relatively smaller changes in propagation direction and tilt angle. What about morphological and geometric evolution of CMEs in the interplanetary space, i.e., from $\sim 20$ Rs to $1 \mathrm{AU}$ ? Much progress has been made in the last one and half decades, thanks to the Heliospheric Imager (HI) (Eyles et al. 2009) onboard STEREO A/B. Nevertheless, the knowledge that has been gathered is largely limited, as discussed below.

Studies of using HI images from a single spacecraft usually assume that CMEs have a constant propagation direction and speed in HI FOVs. Having expanded into a huge volume thus becoming extremely faint, CMEs in $\mathrm{HI}$ images have a much lower signal-to-noise ratio than in coronagraphic images. Instead of forward-fitting CME appearances using a 3D geometric model such as the GCS model, HI studies often make use of timeelongation maps, or so-called J-maps (Sheeley et al. 1999), which are stack plots of slices taken along a given position angle (often along the ecliptic plane) from consecutive images of a single STEREO spacecraft (Lugaz et al. 2009; Davies et al. 2012). The slice provides a direct measure of elongation angles from the inner to the outer edges of HI FOV (Fig. 3). Such time-elongation 
Geometrical modeling for CME on 2012 July 12-14

(a)
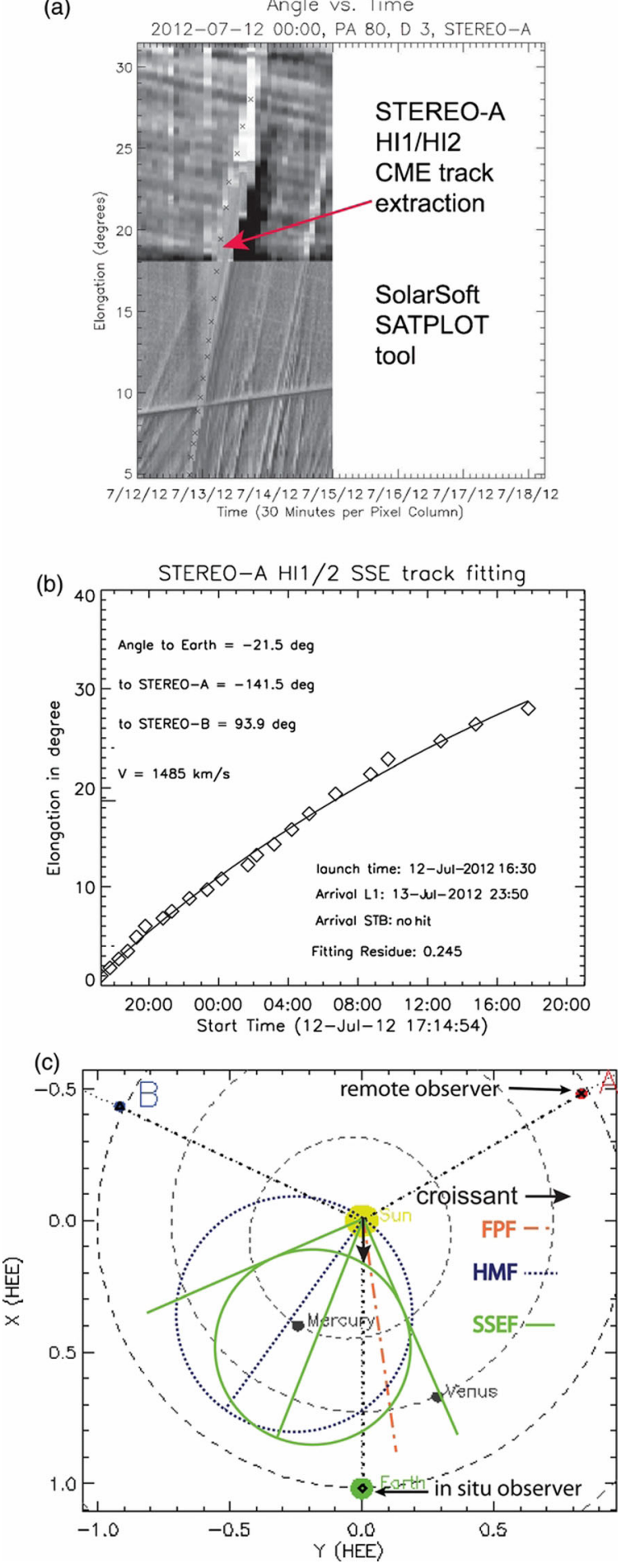

Fig. 3 An example of measuring CME (July 12-14, 2012 CME) leading fronts using slice-stacking plot or J-map and geometric models. a The density track of the CME viable in a J-map from STER EO-A. $\mathbf{b}$ Fits of the extracted CME track with the SSEF (Self-Similar Expansion Fitting) model. c The resulting geometry of the event, with propagation directions derived from FP (dot-dashed red line, $0^{\circ}$ full width), SSEF (solid green line, $90^{\circ}$ full width), and HM (dotted blue line, $180^{\circ}$ full width) geometric assumption of the CME. Adopted from Möstl et al. (2014)

maps show enhanced tracks of the leading edges of a CME, but at the expense of its 3D geometry such as aspect ratios and tilt angles. The time-elongation curve of the tracked feature in the map is then used to determine the propagation longitude and speed of the feature along the selected latitude/slice. This assumption of constant propagation longitude and speed, imposed by this timeelongation map method from a single spacecraft, is not unrealistic, since it is known that most changes have occurred near the Sun in coronagraphic FOVs.

The orbital configuration of STEREO A/B is such that the degree to which the same CMEs are imaged by the HI cameras on both spacecraft critically depends on the mission phase (Harrison et al. 2018). The percentage of such so-called coincident events by HI-1 ranges between $40 \%$ and $90 \%$. Note that the percentage of coincident events by COR2 is about $80 \%$ in total (Vourlidas et al. 2017). For coincident events of HI observations, one can apply a geometric triangulation technique on the timeelongation data from two spacecraft to extract instantaneous propagation longitude and distance at each time of the observation, thus allowing the time variation of propagation longitude and velocity of CMEs (Liu et al. 2010; Lugaz 2010; Davies et al. 2013). Using stereoscopic time-elongation methods and theoretical arguments, several studied suggested possible non-radial motions of CMEs over a large distance in the heliosphere (Lugaz et al. 2010a; Wang et al. 2014; Isavnin et al. 2014).

Nevertheless, caution is needed as the time-elongation methods do not provide a converging result on the propagation longitude when different geometric assumption of CMEs are made (Liu et al. 2013; Davies et al. 2013) and different spacecraft are used (Barnes et al. 2020). Note that a set of commonly used timeelongation methods have been developed, which differ in the assumption of CME geometry on the plane containing the selected slice and the observing spacecraft: as a point or compact source (the fixed- $\phi$, or FP method) (Sheeley et al. 1999; Rouillard et al. 2008), as a circle with the feature at the tangent front and the bottom attached to Sun-center (harmonic mean, or HM method) (Lugaz 2010), or as a generalized circle of certain half angle $\lambda$ (generalized self-expansion, or SEE method) (Davies et al. 2012); the FP and HM geometries form the 
limiting cases with $\lambda$ equals $0^{\circ}$ and $90^{\circ}$ respectively, while $\lambda$ can be chosen between $0^{\circ}$ and $90^{\circ}$ in the SEE method (Fig. 3). Davies et al. (2013) found that the derived CME longitude is a function depending on the choice of $\lambda$, and the disparity in longitudes can be significant between the two limiting cases. In a statistical study of 273 coincident events, Barnes et al. (2020) noted that the longitude derived from single-spacecraft are in fairly poor agreement with each other, and moreover, neither agree well with the results from stereoscopic analysis. Such systematic disparity may indicate the incorrectness of the underlying assumption, i.e., the assumed circular front of CMEs may deviate significantly from the actual morphology, which will be discussed below.

\subsubsection{Morphology of ICMEs in the interplanetary space}

In contrast to the largely self-similar expansion pattern of CMEs in the corona, CMEs may undergo significant deformation in the interplanetary space, thanks to the enhanced effect of structured solar wind flows on CMEs (Odstrčil and Pizzo 1999a; Riley and Crooker 2004). It is understood that, in the regime of high plasma beta where plasma pressure dominates magnetic pressure, the magnetic structure of CMEs will be strongly modulated by the pattern of plasma flow. In the interplanetary space, the solar wind plasma flows along the radial direction but in a spherically diverging geometry. Consequently, such a flow pattern introduces the following kinematic effects on the structure of CMEs: (1) selfsimilar expansion along lateral directions, or directions on the spherical surface (2) no expansion at all along the radial direction that is perpendicular to the spherical surface, leading to the thinning or pancaking of the overall CME morphology (Riley and Crooker 2004). In the following, we discuss the self-similarity of CME evolution along lateral and radial directions respectively.

Observations in HI FOVs show that CMEs maintain a nearly constant angular width, indicating a self-similar expansion or constant aspect ratios along lateral directions with respect to the distance of CMEs (Wood et al. 2009; Wood et al. 2017). Note that the two principal lateral directions for a flux rope CME are along the toroidal and poloidal directions, respectively. To reproduce the observed two-dimensional loop of CME leading fronts in a flexible way, Wood et al. (2009) adopted a geometric shape described by a quasi-Gaussian equation in polar coordinates with a variable power index $\alpha$ regulating the shape of the loop; the shape is a perfect Gaussian for $\alpha=2$, while higher values of $\alpha$ result in loops with flatter tops. Using a statistical survey study of 48 events, Wood et al. (2017) noted that self-similar expansion is a decent, albeit not perfect, approximation for CMEs expanding into the interplanetary space. Such self-similar expansion along lateral directions should have continued from coronagraphic FOVs into HI FOVs.

Nevertheless, the self-similar evolution breaks down for the dimension along the radial direction. The cross section of the CME flux rope can be initially well described by a circle, as in the highly successful GCS models. Into the interplanetary space, the circular shape may evolve into a highly flattened and distorted shape, which has been described as a convex-outward pancake shape (Riley and Crooker 2004), elliptical shape (Savani et al. 2011), or even a concave shape (Savani et al. 2010). As an example of one extreme case, (Savani et al. 2010) clearly showed the observation that a circular-shaped CME in the coronagraphic FOV evolved into a concaved structure in the HI FOV, and suggested that the kinematic effect of a bimodal speed solar wind caused such distortion. Therefore, the shape of the leading front of a CME can deviate significantly from a circular shape, and caution needs to be taken when a circular-shape assumption is assumed in modeling ICMEs.

Note that, besides the studies based on coronal and heliospheric imaging observations mentioned above, the geometry and morphology of CMEs can also be inferred from in situ observations. There is a vast amount of work of fitting in situ data to infer the structure of shocks and magnetic flux ropes, and such studies are partially reviewed in Section 3.5. In the next sub-section, we provide a review on the studies of kinematic properties of CMEs and ICMEs, which are mostly based on the time tracking of the leading fronts of the CME, instead of the 3D extension of the structure.

\subsection{Kinematics of CMEs and ICMEs}

Our knowledge about the whole kinematic evolution of CMEs from the Sun to the Earth has improved significantly in the last decade, largely thanks to the wideangle observations of STEREO. Rising from locations above magnetic polarity inversion lines near the surface of the Sun, CMEs accelerate and reach speeds in the outer corona with a wide range of values from tens of $\mathrm{km} / \mathrm{s}$ up to $\sim 4000 \mathrm{~km} / \mathrm{s}$. The subsequent evolution of CMEs depends on their initial speeds in the outer corona relative to the speed of ambient solar wind: faster CMEs decelerate, while slower CMEs accelerate. As CMEs propagate further into the interplanetary space, their speeds tend to equalize with that of the solar wind due to the effect of aerodynamic drag (or more precisely, magnetohydrodynamic drag). For a large fraction of CMEs, the balance in speed and pressure is not established at the distance of $1 \mathrm{AU}$. The speed of ICMEs at 1 AU ranges from $\sim 300 \mathrm{~km} / \mathrm{s}$ to $\sim 1000 \mathrm{~km} / \mathrm{s}$, meaning that it can be much faster than the ambient solar wind at $1 \mathrm{AU}$. In the following, we provide a review on the Sun-to-Earth kinematic evolution, including the phases 
of evolution, peak velocity, terminal velocity, cessation distance and others. The topic on the prediction of CME Time of Arrival at 1 AU will be given in Section 8.2.

Based on tens of thousands of CMEs observed, it is found that CME speed (i.e., average projected speed measured in LASCO FOV) has a very broad distribution ranging from $\sim 10 \mathrm{~km} / \mathrm{s}$ to $\sim 3000 \mathrm{~km} / \mathrm{s}$ (Yashiro et al. 2004; Robbrecht et al. 2009a; Olmedo and Zhang 2010; Webb and Howard 2012; Lamy et al. 2019). The average speed of all CMEs in the various observed periods is about $300 \mathrm{~km} / \mathrm{s}$ during the solar minimum and about $500 \mathrm{~km} / \mathrm{s}$ during the solar maximum. Further, halo CMEs, which are the ones likely hitting the Earth, have an average speed of about $950 \mathrm{~km} / \mathrm{s}$, or about twice of that of all CMEs. Slow CMEs are quite common, as about half of CMEs in the LASCO FOV are slower or near the speed of the ambient solar wind. On the other hand, fast CMEs are equally common. Nevertheless, extremely fast CMEs, i.e., $>1500 \mathrm{~km} / \mathrm{s}$, are rather rare, occupying $~ 0.5 \%$ of all CMEs (Wang and Zhang 2007). The highest CME speed on the record is $\sim 4400 \mathrm{~km} / \mathrm{s}$ (Gopalswamy et al. 2018a, b, c, d, e).

Recently, Barnes et al. (2019) made a statistical study of CME kinematics in the STEREO HI-1 FOV and compared with that in the LASCO FOV. They found that the velocity distributions are similar in both areas: a sharp peak at the low end of the distribution and a long tail of high-speed CMEs. The yearly mean speeds in HI-1 FOV are consistently higher than that in LASCO; however, the two types of speeds are very similar after projecting $\mathrm{HI}$ speeds onto the plane of the sky. In the $\mathrm{HI}$ FOV, the range of CME speeds is from $\sim 100 \mathrm{~km} / \mathrm{s}$ to $\sim 2000 \mathrm{~km} / \mathrm{s}$. It is noticed that there are very few CMEs with speeds less than $200 \mathrm{~km} / \mathrm{s}$ in the HI FOV, which is of a distinct contrast with that of LASCO CMEs. This difference is certainly not surprising, as slow CMEs in the corona are picked up by the drag of ambient solar wind.

A large number of studies on individual events have provided detailed kinematic evolution of CMEs from corona and far into the inner heliosphere (Wood and Howard 2009; Poomvises et al. 2010; Liu et al. 2010; Colaninno et al. 2013; Hess and Zhang 2014; Liu et al. 2016; Wang et al. 2016a, b, c, d; Wood et al. 2017). The observed speed profiles of three typical CMEs, which are of slow, intermediate and fast initial speeds respectively, are shown in Fig. 4 (adopted from Liu et al. 2016). Clearly, faster CMEs decelerate and slower CMEs accelerate, as also shown in earlier studies (Sheeley et al. 1999; Gopalswamy et al. 2000). One of the interesting results from observational studies is that there appears the existence of a cessation distance, at which a CME reaches its terminal velocity; after this distance, the CME moves at a nearly constant speed, or too small to be measured by existing imaging instruments. Note that we are cautious on the usage of the term "terminal speed," as CME speeds will continue to change, albeit in a relatively small rate (e.g., $<1 \mathrm{~m} / \mathrm{s}^{2}$ ). Poomvises et al. (2010) showed that this cessation distance was at about $50 \mathrm{Rs}$ for several events including very fast ones. Using a kinematic model that divides the CME evolution into 24 phases of constant acceleration and constant velocity (Wood and Howard 2009), a recent statistical study by Wood et al. (2017) showed that the cessation distance ranged from $\sim 10$ Rs to $\sim 100$ Rs, and the terminal velocity ranged from $\sim 300 \mathrm{~km} / \mathrm{s}$ to $\sim 1200 \mathrm{~km} / \mathrm{s}$. Similar result was found in an earlier study based on Type II radio observations (Reiner et al. 2007).

CMEs reach their peak velocity at varying heights from the Sun. In general, fast CMEs reach their peak velocity at a low height, thanks to strong and impulsive acceleration, while slow CMEs reach their peak velocity at a relatively high height (Zhang and Dere 2006; Bein et al. 2011, 2012; Wood et al. 2017). Based on a statistical study of 95 events, Bein et al. (2011) found that the heights of peak velocity distribute from a very low height of 1.17 Rs (from disk center) to $\sim 10.5$ Rs (close to the border height of STEREO COR2 used in this study). A continued study by Bein et al. (2012) found that CMEs associated with flares, in comparison with CMEs associated with filaments, have on average significantly higher peak acceleration and lower height of peak velocities. Wood et al. (2017) found that the average peak velocity height was $\sim 3.2$ Rs for fast CMEs that were associated with flares, $\sim 13.9$ Rs for intermediate velocity CME associated with erupting filaments, and $\sim 29.4$ Rs for slow CMEs that were not associated with any apparent surface source regions.

The full Sun-to-Earth evolution of CMEs can be largely divided into four phases, each of which depends how the velocity varies and what forces drive the velocity change. Near the surface and in the corona, the full kinematic evolution of a CME can be characterized by three distinct phases: (1) a slow rise phase, or initiation phase, (2) a fast acceleration phase, or main phase, (3) a propagation phase with no or small variation of velocity (Zhang et al. 2001); this third phase is called residual acceleration phase in Zhang and Dere (2006). During the first two phases, a CME should be mainly driven by the Lorentz force. However, following the main acceleration, the Lorentz force may become significantly weaker, and the aerodynamic drag force sets in and become important. During this third phase, a CME likely experiences a combined effect of both Lorentz force and aerodynamic drag force, leading to the observed residual acceleration which can be either positive or negative (Zhang and Dere 2006). Further moving out, the Lorentz force eventually diminishes and the aerodynamic drag force will 


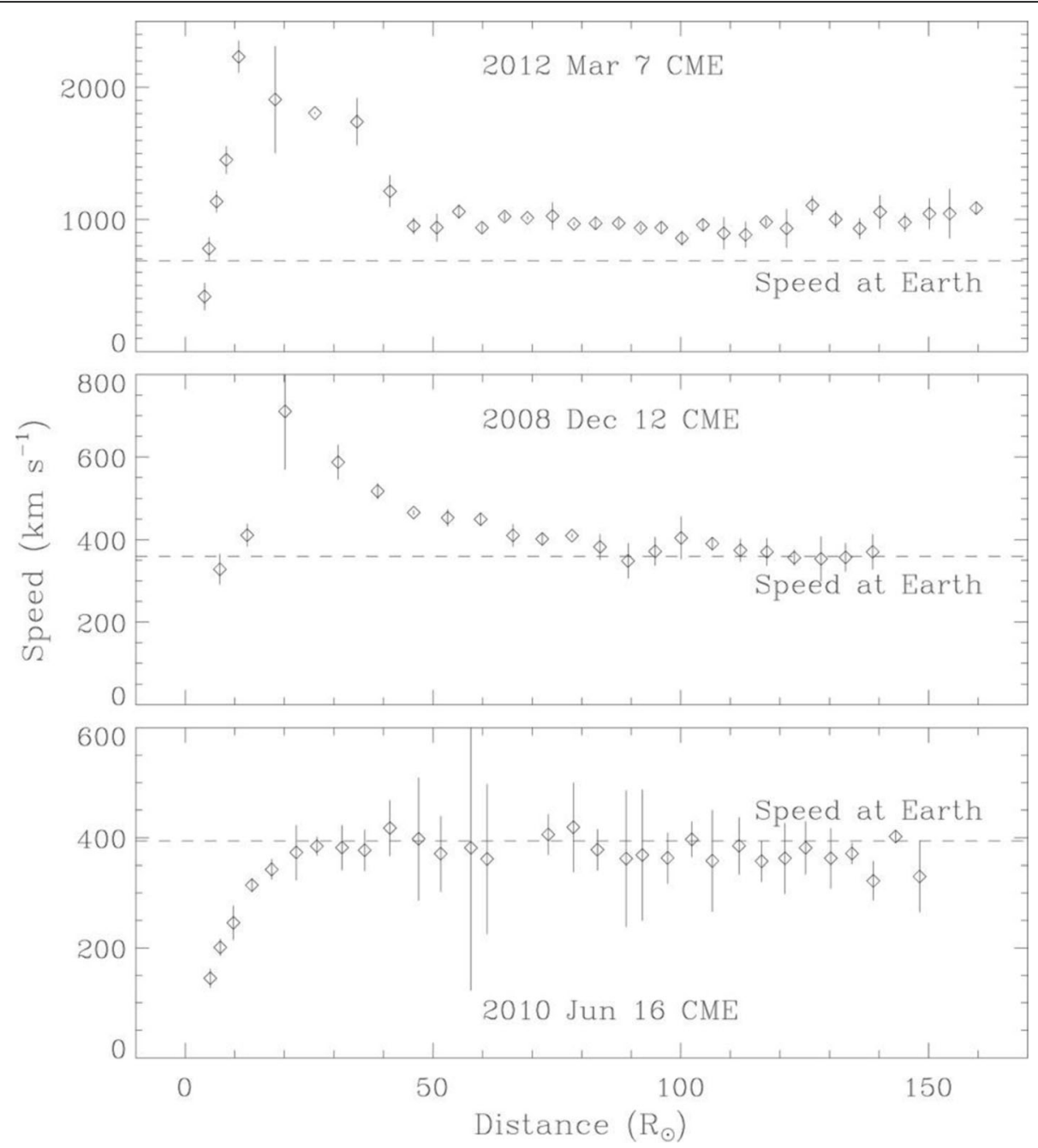

Fig. 4 Sun to Earth velocity profiles of a typical fast CME (upper), a typical intermediate-speed one (middle), and a typical slow one (lower). The horizontal dashed line indicates the observed speed at the Earth. Adopted from Liu et al. (2016)

dominate; this phase can be considered the 4th phase of the full evolution, or the drag phase. When only the aerodynamic drag force is considered, the kinematic evolution of a CME can be modeled in a relatively straightforward way (Cargill 2004; Vršnak et al. 2013). As the aerodynamic drag force is proportional to the square of the difference between the CME velocity and the ambient solar wind velocity, the CME velocity will asymptotically approach the velocity of the ambient solar wind. In other words, a faster CME decelerates and a slower CME accelerates, and the acceleration rate is not a constant but asymptotically approaches zero. For a slow CME, the full evolution may be reduced to only two phases, a gradual acceleration out to about 20-30 Rs, followed by a nearly constant speed near the solar wind level (Liu et al. 2016). A detailed review on theories of CMEs propagation is given in Section 3.

\subsection{Coronal sources of solar eruptions}

The initiation and early evolution of CMEs cannot be observed using traditional coronagraphic observations, due to the blockage of the eruption region by the occulting disk. Therefore, various associated phenomena in $\mathrm{H} \alpha$, extreme-ultraviolet (EUV), X-rays, and microwaves on solar disk are linked to specific properties of the eruption and used to infer the origin of CMEs (Gopalswamy et al. 1999; Hudson and Cliver 2001; Harra 2009; Webb and Howard 2012). Over the course of the eruption, the associated activities can be a combination of filament eruptions, solar flares, large-scale coronal EIT waves, post-eruptive arcades, and coronal dimmings. For example, the CME onset is often accompanied with the eruption of filaments/prominences that later form the inner bright core of CMEs observed in coronagraphic data (Gopalswamy et al. 2003; Parenti 2014). The relationship between eruptive prominences and 
CMEs was investigated in several statistical studies (Munro et al. 1979; Webb and Hundhausen 1987; Hori and Culhane 2002; Gopalswamy et al. 2003), where an association rate of up to $90 \%$ was found.

Low coronal observations also revealed the close relationship between solar flares and CMEs

(Schmieder et al. 2015; Vršnak 2016). Strong and powerful flares tend to be associated with fast and massive CMEs (Moon et al. 2002; Burkepile et al. 2004; Vršnak et al. 2005; Bein et al. 2012), which results in a 90\% correspondence for flares above X-class (Yashiro et al. 2006). However, there exist flares without CMEs (i.e., confined flares (e.g., Pallavicini et al. 1977; Wang and Zhang 2007; Sun et al. 2015) and vice versa, CMEs without flares (e.g., stealth CMEs) (Robbrecht et al. 2009b; Ma et al. 2010; Howard and Harrison 2013; D'Huys et al. 2014). A recent study by Nitta and Mulligan (2017) showed that stealth CMEs can result in significant geoeffective disturbances at $1 \mathrm{AU}$, highlighting their importance in space weather research.

If CMEs and flares occur together, they are interpreted to be different parts of the same magnetically driven event (Harrison 1995; Priest and Forbes 2002; Webb and Howard 2012; Green et al. 2018).

Over the past years, it was shown that CMEs and flares are closely related in time; i.e., the SXR peak and the main acceleration phase of the CME are nearly synchronized (Zhang et al. 2001; Neupert et al. 2001; Shanmugaraju et al. 2003; Maričić et al. 2004; Vršnak et al. 2004; Zhang et al. 2004; Zhang and Dere 2006; Cheng et al. 2020). The main acceleration phase of the CME is correlated with the time evolution of the flare-related hard X-ray burst (Temmer et al. 2008; Gou et al. 2020) and a close relationship between their onset times was found in statistical studies (Maričić et al. 2007; Bein et al. 2012). Further evidence for a close flare/CME relationship is provided by the strong correlation between characteristic CME parameters, such as the velocity, the acceleration, and its kinetic energy with the SXR peak flux, indicating the flare strength, or the integrated flux of the associated flare (Vršnak et al. 2005; Maričić et al. 2007; Yashiro and Gopalswamy 2009).

Since the flare energy release rate is closely related to the magnetic reconnection rate (Miklenic et al. 2009), a feedback relationship between the CME and its associated flare is established (Zhang et al. 2001; Vršnak 2008; Temmer et al. 2010; Welsch 2018). Increasing reconnection rates enhance CME acceleration, and vice versa, enhanced acceleration provides more efficient reconnection. Studies showed the correlation between CME velocities and the total reconnection flux supporting this interpretation (Qiu and Yurchyshyn 2005; Miklenic et al. 2009; Tschernitz et al. 2018; Gopalswamy et al. 2018a, b, c, d; Pal et al. 2018). The most recent study by Zhu et al. (2020) even directly proves this interpretation observationally by reporting on a strong correlation between the reconnection rates, estimated by flare ribbons and CME accelerations $(c>0.7)$. Interestingly, they also report on a positive correlation between the maximum speed of CMEs and the total reconnection flux but only for fast CMEs $(v>600 \mathrm{~km} / \mathrm{s})$. For slow CMEs with weak reconnection, other physical processes may play a more important role during acceleration than magnetic reconnection.

The initial lateral expansion of the CME also drives fast-mode magneto-sonic waves observed as large-scale perturbations of enhanced EUV emission, so-called EIT waves (Thompson et al. 1999; Patsourakos and Vourlidas 2009; Long et al. 2016). Their speeds typically range from 200 to $400 \mathrm{~km} / \mathrm{s}$ (Klassen et al. 2000; Thompson and Myers 2009; Muhr et al. 2014), but also EIT waves with speeds up to $1000 \mathrm{~km} / \mathrm{s}$ have been reported (Nitta et al. 2013; Seaton and Darnel 2018). Statistical studies revealed that fast and wide CMEs are in general accompanied with well-observed EIT waves often associated with shocks and therefore also related with type-II radio bursts (Biesecker et al. 2002; Cliver et al. 2005; Nitta et al. 2013, 2014; Muhr et al. 2014; Warmuth 2015). Combining type II radio burst observations with EUV waves observed by SOHO and STEREO, Gopalswamy et al. (2013b) found that the EUV waves are shocks forming very close to the Sun - as low as 0.2 Rs above the solar surface. For the physical mechanisms leading to the shock wave formation and coronal and chromospheric response, see, e.g., Vršnak et al. (2016) and references therein.

After the CME has erupted, bright post-eruptive arcades or post-flare loops appear in soft X-ray and EUV (Kahler 1977; McAllister and Hundhausen 1996; Tripathi et al. 2004) as a consequence of magnetic reconnection processes (Kopp and Pneuman 1976). Tripathi et al. (2004) statistically analyzed post-eruptive arcades using data from $\mathrm{SOHO} / \mathrm{EIT}$. They found that the majority of post-eruptive arcades (92\%) were associated with CMEs identified in SOHO/LASCO.

Due to the expansion of the CME volume and evacuation of plasma during the eruption, regions of decreased emission in soft X-rays and EUV are formed, so-called coronal dimmings (Hudson et al. 1996; Thompson et al. 2000; Harra and Sterling 2001; Vanninathan et al. 2018). As they represent the lower footprint of CMEs in the low corona, their properties are closely related to the initial properties of the observed CME later on. For instance, several studies tried to relate the mass loss within coronal dimming regions to the CME mass measured from coronagraphic observations (Harrison and Lyons 2000; Zhukov and Auchère 2004; Aschwanden et al. 2009; López et al. 2019). 
Recently performed statistical studies confirm the close connection between coronal dimmings and CMEs and found that the dimming area, its total magnetic flux, and its brightness are strongly correlated with the CME mass (Dissauer et al. 2018, 2019; Sindhuja and Gopalswamy 2020). Dimming parameters, describing its dynamics, such as the area growth rate, brightness change rate, and magnetic flux change rate, are tightly related to the CME speed. This is in agreement with the results of Mason et al. (2016) who studied coronal dimmings extracted from full-disk irradiance light curves of SDO/ EVE (EUV Variability Experiment).

A number of studies also successfully compared magnetic flux rope properties, such as the magnetic flux, the chirality, and its helicity sign determined from posteruptive arcades, flare ribbons, and coronal dimmings measured close to the Sun with magnetic cloud properties at 1 AU (Qiu et al. 2007; Yurchyshyn 2008; Hu et al. 2014; Marubashi et al. 2015; Gopalswamy et al. 2017b; Palmerio et al. 2017, 2018; James et al. 2017; Aparna and Martens 2020). The total amount of magnetic flux ejected during an eruption is estimated by the total reconnection flux in the wake of the CME or sometimes also by the magnetic flux involved in coronal dimming regions, which form the footprint of CMEs in the low corona (Mandrini et al. 2005; Attrill et al. 2006; Qiu et al. 2007; $\mathrm{Hu}$ et al. 2014). Especially the total reconnection flux strongly correlates with the magnetic flux of magnetic clouds (Qiu et al. 2007; Hu et al. 2014).

The helicity sign and the total amount of helicity of magnetic clouds at $1 \mathrm{AU}$ seem to be strongly controlled by the location and properties of the solar source region (Cho et al. 2013; Hu et al. 2014; Marubashi et al. 2015). CMEs erupting in the southern (northern) hemisphere tend to have a positive (negative) helicity sign (hemispheric helicity rule, e.g., Pevtsov et al. 2003). Recently, Aparna and Martens (2020) investigated the directionality (chirality) of 86 CMEs-ICME pairs by comparing the orientation of their flux rope axes close to the Sun with the direction of the interplanetary magnetic field near Earth at L1. An agreement between the northward/ southward orientation of $\mathrm{Bz}$ between ICMEs and their CME source regions was found in $85 \%$ of the cases, which is comparable to earlier results by Palmerio et al. (2018) and Yurchyshyn (2008), which found agreement for $55 \%$ and $77 \%$ of their cases.

In recent years, several studies also focused on Sun-toEarth analysis of CMEs by linking the low coronal behavior and properties of the eruption with its observed in situ signature (Möstl et al. 2015; Patsourakos et al. 2016; D'Huys et al. 2017; Temmer et al. 2017a). A number of studies also compared magnetic flux rope properties, such as the magnetic flux, the chirality, and its helicity sign determined from post-eruptive arcades, flare ribbons, and coronal dimmings measured close to the Sun with magnetic cloud properties at 1 AU (Qiu et al. 2007; Gopalswamy et al. 2017b; Palmerio et al. 2017; James et al. 2017). Scolini et al. (2019b) used proxies of magnetic flux estimates determined from post-flare arcades (Gopalswamy et al. 2017b), flare ribbons (Kazachenko et al. 2017; Tschernitz et al. 2018) as well as coronal dimmings (Dissauer et al. 2018), as initial input for the global heliospheric EUHFORIA model, to study the geoeffectiveness of the famous 2017 September events. Good agreement with the observed Dst profile was found for simulations using the optimized input and including CME-CME interactions.

\subsection{Solar cycle variations of CMEs and ICMEs}

Solar cycle 24 is known to be weaker than previous several solar cycles, which is the focus of many studies during the VarSITI program. A weak solar cycle 24 is understood to be due to the weak polar magnetic field in the preceding solar minimum according to the Babcock Leighton Mechanism of the solar cycle (see e.g., Petrovay 2010). A weak cycle implies mild space weather that helps satellites in Earth orbit live longer. A weak cycle also means less total solar irradiance reaching Earth (e.g., Krivova and Solanki 2008). Here, we focus on the effect of a weak solar cycle on solar wind magnetic structures originating from the Sun and their space weather consequences.

Both solar source and impact of CMEs showed significant variations in cycle 24. The overall rate of CMEs increased in solar cycle 24 relative to cycle 23 , although the rate of fast and wide CMEs decreased. Accordingly, the phenomena that are linked to fast and wide (FW) CMEs appeared subdued in cycle 24. The rate of occurrence of CMEs is known to be correlated with the sunspot number (SSN) for a long time. However, the slope of the regression line is significantly different in cycle 24 . The relation between CME width and speed is also different in cycle 24: for a given speed, cycle-24 CMEs are significantly wider. CMEs are the main source of severe space weather. Weakened solar activity is reflected in the weak heliospheric state in terms of magnetic field strength, temperature, density, speed, and consequently the total pressure. The backreaction of the weakened heliosphere had led to the changed properties of CMEs and hence affected the space weather consequences. Marked reductions are observed in the number of intense (Dst $\leq-100 \mathrm{nT}$ ) geomagnetic storms and highenergy $(\geq 500 \mathrm{MeV})$ solar energetic particle (SEP) events. The number of halo CMEs in cycle 24 did not decrease significantly. In fact, the number of halo CMEs normalized to the sunspot number is larger in cycle 24. One would have expected enhanced geomagnetic activity in cycle 24 because of the higher abundance of halo CMEs, 
but it did not occur. In this section, we summarize some of the key observational results that describe the compound effect of the weak solar activity and heliospheric backreaction on CME properties.

\subsubsection{Solar activity and eruption properties}

Figure 5 shows the solar-cycle variation of CMEs and flares compared to SSN cycles 23 and 24 updated from (Gopalswamy et al. 2020a; Gopalswamy et al. 2020b). We can readily infer the following: (i) the daily rate of the general population of CMEs (width $\geq 30^{\circ}$ ) and that of the soft $\mathrm{X}$-ray flares (size $\geq \mathrm{C} 1.0$ ) did not decline in cycle 24, (ii) the FW CME rate declines significantly in cycle 24 (as opposed to the general population), (iii) the CME daily rates have a different relationship with SSN in the two cycles, (iv) the variation in the number of FW CMEs is similar to that of major soft X-ray flares ( $\mathrm{M}$ - and X-class flares), and (v) the CME rate increases more rapidly as the activity increased, indicated by the steeper slope in the cycle- 23 CME rate-SSN scatter plot. The reduction in FW CMEs is significant because they are the ones that are relevant for space weather consequences (geomagnetic storms and SEP events).

Petrie (2015) studied SOHO/LASCO CMEs with angular widths $>30^{\circ}$ listed in the manual (CDAW) and automatic (CACTus and SEEDS) catalogs. He found that the CME rate relative to the sunspot number began an upward divergence with respect to the SSN in 2004 after the polar field reversal, while the interplanetary magnetic field decreased by $\sim 30 \%$ around the same time (see Fig. 3a). These results are consistent with the enhanced halo CME detections due to the increased CME expansion in a heliosphere with diminished total pressure (Gopalswamy et al. 2015a). Petrie (2015) also showed that the increased CME rate in cycle 24 is not due to the
LASCO cadence change that occurred in August 2010 (Wang and Colaninno 2014; Hess and Colaninno 2017). The cadence change was also found to be not important for halo CME rates (Gopalswamy et al. 2015a). Michalek et al. (2019) showed that the higher rate of the narrow CMEs can be attributed to the global magnetic structure in cycle 24 coupled with the reduced total pressure in the heliosphere, in agreement with Petrie (2015).

\subsubsection{Phenomena associated with energetic CMEs}

Figure 6 shows the solar cycle variation of the numbers of halo CMEs, intense geomagnetic storms, large SEP events, and decameter-hectometric (DH) type II bursts. All the numbers are summed over Carrington rotation periods. The properties of halo CMEs have a good overlap with FW CMEs, although some halos are wide, but not fast. Halo CMEs, when front-sided, are important because they can affect space weather. Intense geomagnetic storms are mostly due to energetic CMEs heading toward Earth containing southward magnetic field components either in the ejecta part or in the shock sheath. On the other hand, the shock at the leading edge of CMEs accelerates electrons and ions. The accelerated electrons produce type II radio bursts that are a good indicator of shock-driving CMEs near the Sun and in the interplanetary medium. Accelerated particles traveling along interplanetary field lines are detected as SEP events. We see that the number of events in each case generally follows the solar cycle with more events occurring during solar maxima. A notable exception is the number of intense geomagnetic storms in cycle 24 that remained flat.

2.5.2.1 Halo CMEs in solar cycle 24 As reported in (Gopalswamy et al. 2015a; Gopalswamy et al. 2020b; Gopalswamy et al. 2020), the halo CME rate in cycle 24
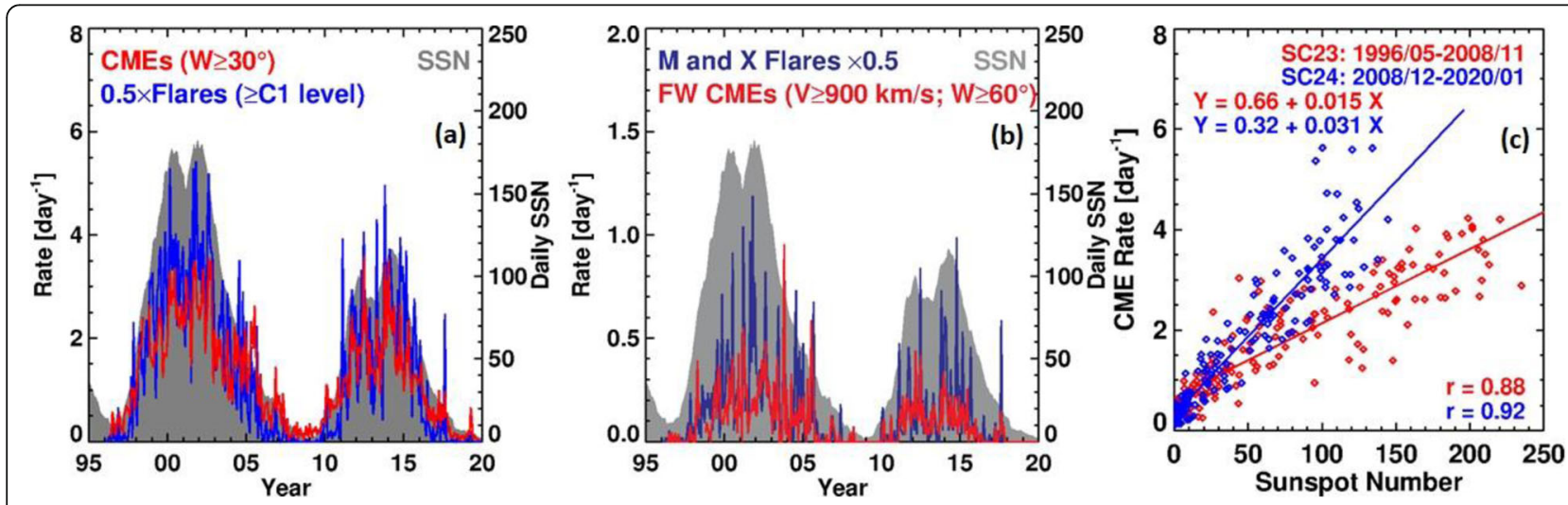

Fig. 5 Solar cycle variation of eruptive phenomena (flares and CMEs): a daily CME rate for the general population (width $\geq 30^{\circ}$ ) in red and the number of soft X-ray flares with size $\geq C 1.0$ in blue, $\mathbf{b}$ fast and wide CMEs (speed $\geq 900 \mathrm{~km} / \mathrm{s}$; width $\geq 60^{\circ}$ ) in red and major ( $\mathrm{M}$ - and X-class) soft X-ray flares in blue, and c scatter plots between the sunspot number (SSN, V2.0) and the daily CME rate (general population) for cycle 23 (red) and 24 (blue). In (a) and (b), SSN is shown in gray background. The CME and flare rates are averaged over the Carrington rotation (CR) periods. The flare rates are multiplied by 0.5 to fit the scale. Updated from Gopalswamy et al. (2020a); Gopalswamy et al. (2020b) 

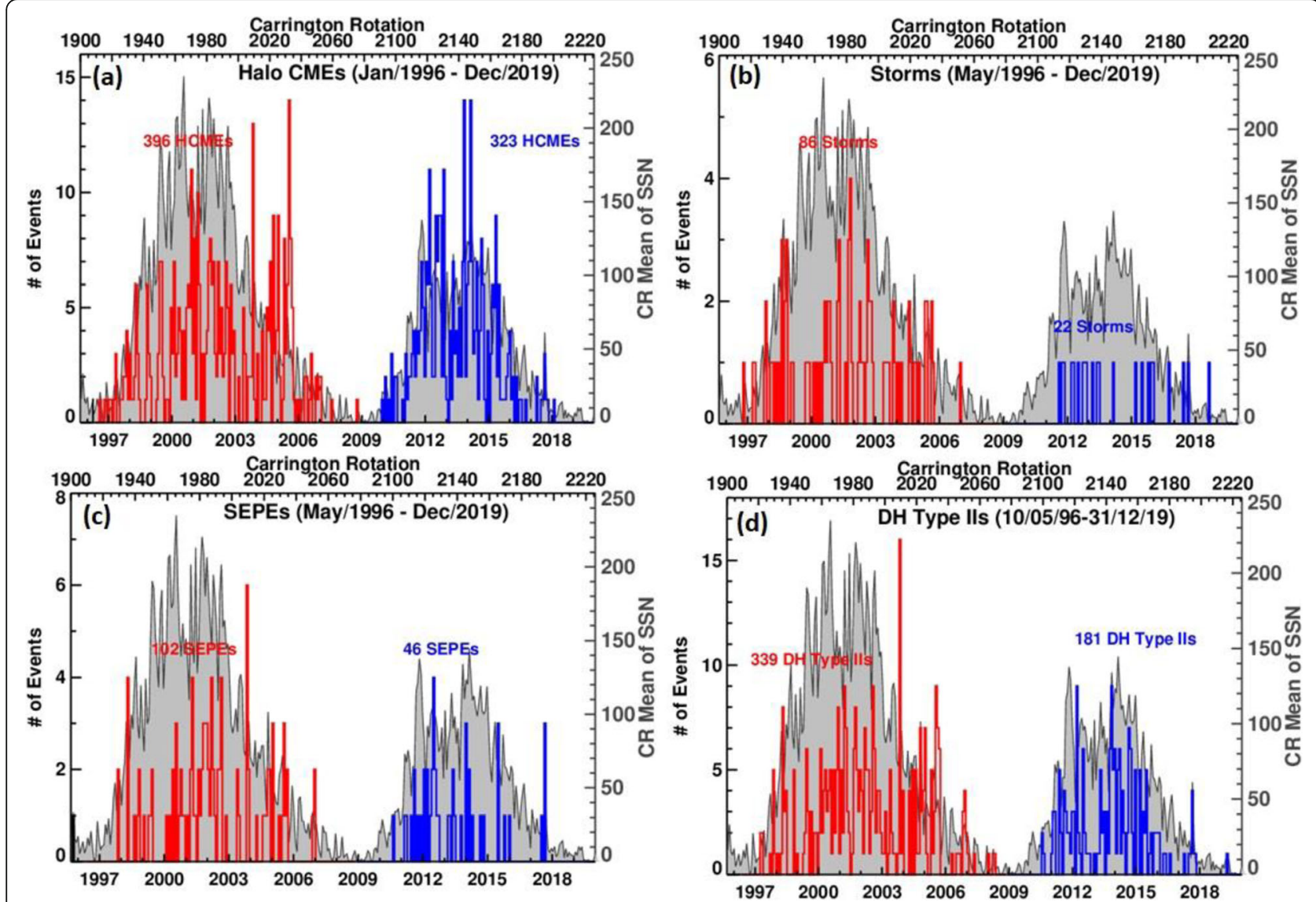

Fig. 6 Solar cycle variation of the key CME-related phenomena during cycles 23 and 24 (from 1996 to the end of 2019): a SOHO/LASCO halo CME number from https://cdaw.gsfc.nasa.gov/CME_list/halo/halo.html. b the number of large geomagnetic storms (Dst $\leq-100 \mathrm{nT}$ ), $\mathbf{c}$ the number of large solar energetic particle events (SEPEs) detected in the $>10 \mathrm{MeV}$ GOES channel with intensity exceeding $10 \mathrm{pfu}$, and $\mathbf{d}$ the number of decameter-hectometric $(\mathrm{DH})$ type II radio bursts from Wind/WAVES. For comparison with the solar cycle, the sunspot number averaged over Carrington rotation (CR) periods is also shown. Updated from Gopalswamy et al. (2015a, b, c)

did not decline commensurate with the SSN. There are roughly the same number of halos in the two cycles. When normalized to SSN, cycle 24 has 30\% more halos (per SSN). As with the general population of CMEs, the halo CMEs are slower in cycle 24. Furthermore, CMEs in cycle 24 become halos at shorter heliocentric distances than in cycle 23. These authors attributed the peculiar behavior of halo CMEs to the diminished heliospheric total pressure in cycle 24 that made relatively slower CMEs and those originating at larger central meridian distances become halos. Solar wind parameters measured at Sun-Earth L1 confirm that most of the parameters have lower values in cycle 24. In particular, the decline in the heliospheric field strength resulted in the diminished total pressure and the Alfven speed (Gopalswamy et al. 2020a).

2.5.2.2 Interplanetary CMEs in solar cycle 24 The reduction in the number of FW CMEs in cycle 24 is expected to be reflected in the number of interplanetary
CMEs (ICMEs) observed at $1 \mathrm{AU}$ because the latter are known to be associated with energetic CMEs. The annual number of ICMEs in Fig. 7 shows clear solar-cycle variation, similar to other phenomena. The total number of ICMEs declined from 307 in cycle 23 to 208 in cycle 24 , representing a reduction of $32 \%$. The decline is smaller than that in SSN. One possible explanation is that ICMEs also originate from non-spot regions where quiescent filaments erupt and the associated CMEs become ICMEs. When normalized to the cycle-averaged SSN in each cycle (81 in cycle 23 dropping to 49 in cycle 24 ), we see a $12 \%$ increase in the number of ICMEs. If we separate the ICMEs into magnetic clouds (MCs) and non-cloud ejecta (EJ), we see a similar trend, but the decline in EJ (37\%) is steeper than in MCs (25\%). When normalized to SSN, we see an increase of $4 \%$ and $25 \%$ for EJs and MCs, respectively. This behavior was noted previously for MCs detected during the rise and maximum phases of cycles 23 and 24 (Gopalswamy et al. 2015a, b, c). The fraction of MCs is also slightly higher 

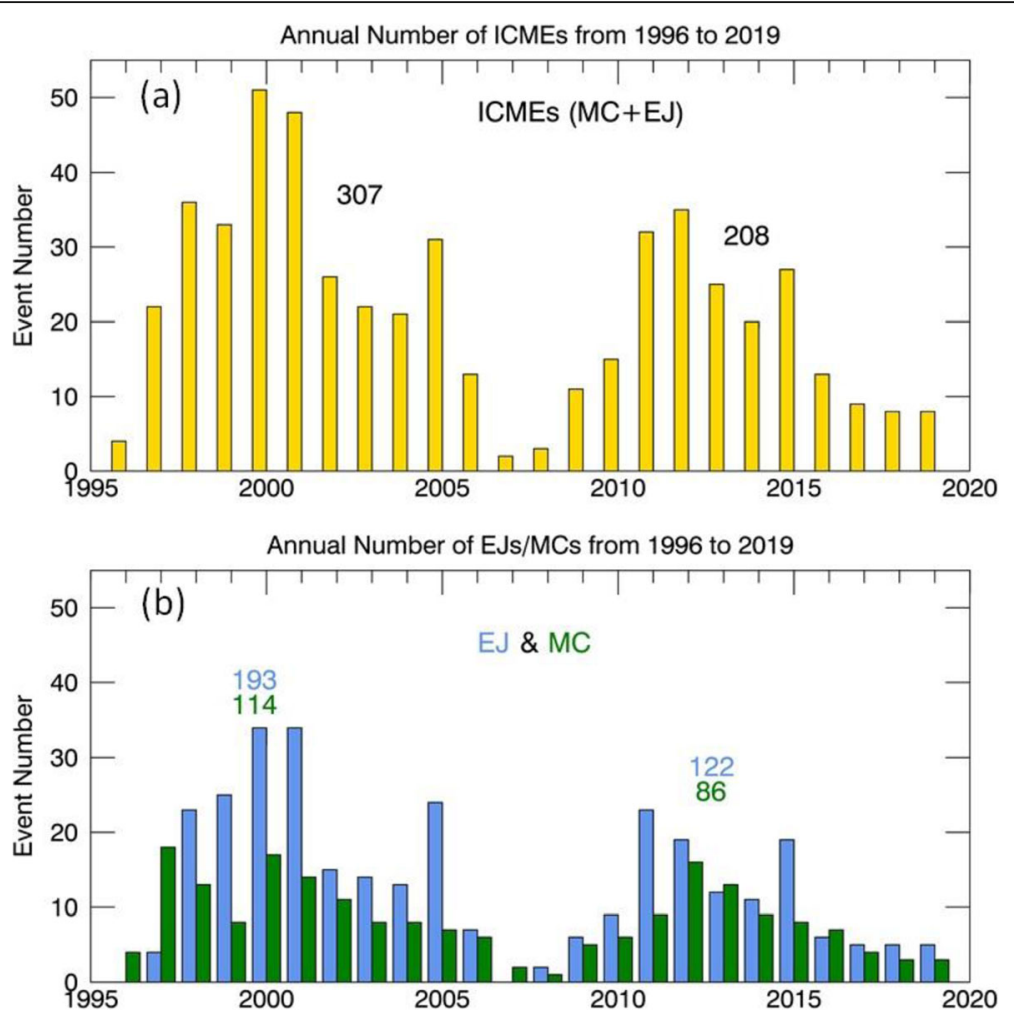

Fig. 7 a Solar cycle variation of the annual number of ICMEs (magnetic clouds—MCs and non-cloud ejecta—EJs) observed near Earth as compiled in http://www.srl.caltech.edu/ACE/ASC/DATA/level3/icmetable2.htm. The total number of ICMEs in cycles 23 (307) and 24 (208) are noted on the plot. $\mathbf{b}$ Annual number of EJs and MCs shown separately. The number of EJs and MCs is noted on the plot

in cycle $24: 37 \%$ (114 out of 397 ICMEs) vs. $41 \%$ (86 out of 208 ICMEs) in cycle 23.

\subsubsection{Stream interaction regions}

While CMEs cause most of the intense geomagnetic storms, stream interaction regions (SIRs) and especially co-rotating interaction regions (CIR) can cause relatively weaker but more frequent geomagnetic storms. The solar sources of SIRs and CIRs also have been shown to be different in cycles 23 and 24. Nakagawa et al. (2019) studied the temporal and spatial variations of the lowlatitude coronal hole $(\mathrm{CH})$ area related to high-speed solar (HSS) wind during solar cycles 23 (1996-2008) and 24 (2009-2016). They found that (i) the CHs in solar cycle 24 appeared over a wider latitude range than in solar cycle 23 , and (ii) the maximum values of the $\mathrm{CH}$ area and the solar wind speed in solar cycle 24 were smaller than those in solar cycle 23. Jian et al. (2019) compared the annual occurrence rates of SIRs and CIRs using Wind/ACE and STEREO in situ observations from 1995 to 2016 as displayed in Fig. 8. They found a higher occurrence rate of SIRs in cycle 24, which they attribute to the presence of persistent equatorial coronal holes as well as weaker CMEs in that cycle. The fraction of CIRs is higher in the declining minimum phase of each cycle.
Grandin et al. (2019) developed a catalog of 588 HSS and SIR events that occurred during the interval 1995 to 2017. Their list is largely in agreement with the list of SIRs identified manually by Jian et al. (2019).

\subsubsection{Geoeffectiveness}

A severe reduction in the geoeffectiveness of CMEs in cycle 24 as measured by the number of intense (Dst $\leq-$ $100 \mathrm{nT}$ ) geomagnetic storms has been reported (Gopalswamy et al. 2014a, b; Gopalswamy et al. 2015a, b, c) in the rise to the maximum phases. In the updated version covering two full cycles shown in Fig. 4b, we see that there are 86 intense storms in cycle 23 compared to just 22 in cycle 24 . These include 11 CIR storms in cycle 23 and 2 in cycle 24 (an 82\% reduction). The first intense geomagnetic storm occurred during the maximum phase of cycle 24 on 2013 June 1 due to a low-latitude coronal hole that was at the central meridian on 2013 May 30 (Gopalswamy et al. 2015c). The second storm occurred on 2015 October 6 due to a coronal hole that was present at the central meridian on 2015 October 5 (Watari 2018). The number of intense storms due to CMEs declined by 73\% from 75 to 20 . Both these reductions can be attributed to the dilution of the CME and CIR magnetic content. 


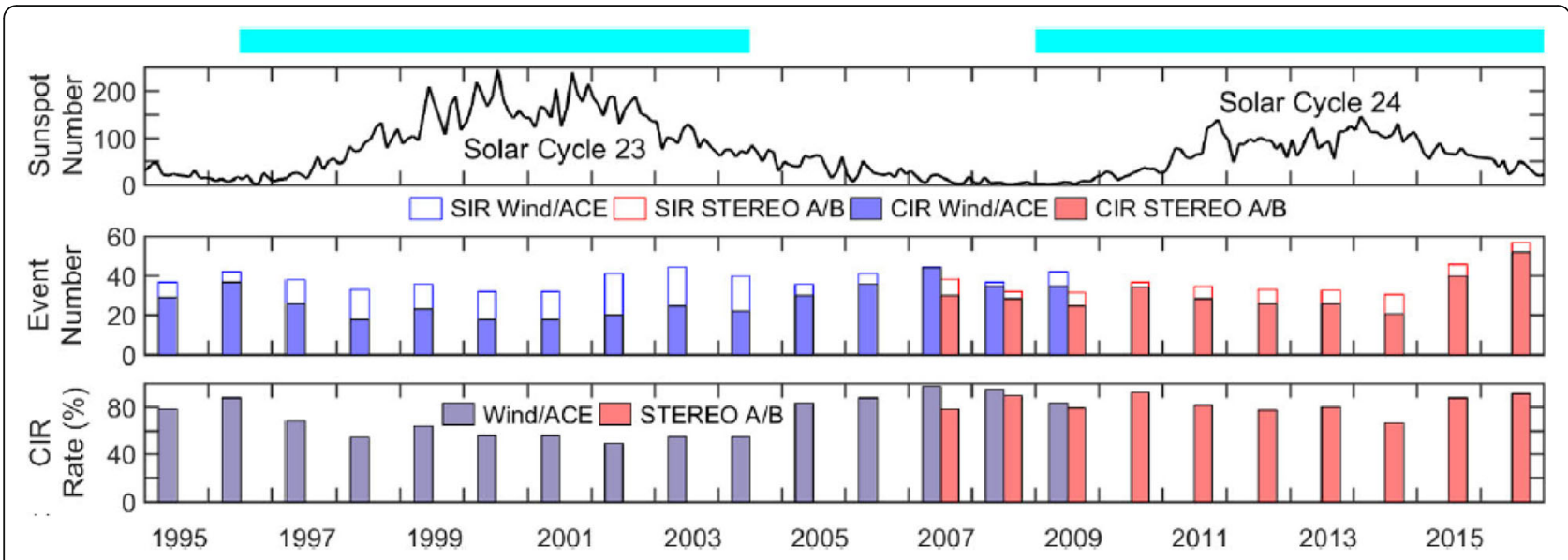

Fig. 8 Monthly sunspot number (top), number of SIR/CIR (middle), and CIR rate (bottom) in solar cycles 23 and 24. Corresponding epochs in cycles 23 and 24 are indicated by the cyan bars at the top (adapted from Jian et al. 2019)

Considering MCs that occurred during the first 6 years in each of cycles 23 and 24, it was found that the MCassociated storms were weaker in cycle 24: the average value of Dst increased from - 66 to $-55 \mathrm{nT}$. Scolini et al. (2018b) investigated the geoeffectiveness of halo CMEs in cycles 23 and 24 . They found that during the first 85 months of Cycle 23 the geoeffectiveness rate of the disk-center full-halo CMEs was $58 \%$ compared to $35 \%$ in cycle 24 . The average minimum value of the Dst index was - $146 \mathrm{nT}$ in cycle 23 compared to $-97 \mathrm{nT}$ in cycle 24 . These results are consistent with the reduced geoeffectiveness of cycle-24 MCs (Gopalswamy et al. 2015a, b, c). Hess and Zhang (2017) studied 70 Earthaffecting ICMEs in Solar Cycle 24 and found the lack of events resulting in extreme geomagnetic storms.

Selvakumaran et al. (2016) considered the moderate and intense geomagnetic storms that occurred during the first 77 months of solar cycles 23 and 24 . While they confirmed an $80 \%$ reduction in the occurrence of intense storms, they found that the number of moderate storms decreased by only $\sim 40 \%$. When moderate storms from CIRs are considered separately, these authors found that the reduction in the number of geomagnetic storms is more drastic (see also Grandin et al. 2019). In cycle 23, there were $43 \mathrm{CIR}$ storms during the first 77 months of cycle 23 compared to 15 in cycle 24 , amounting to a reduction of $63 \%$. This is about the same when the CIR storms are separated into moderate and intense storms. Chi et al. (2018a) studied the geoeffectiveness of SIRs from 1995 to 2016 and found that about 52\% of the SIRs caused geomagnetic storms with Dst $\leq-30 \mathrm{nT}$, but only 3\% of them caused intense geomagnetic storms (Dst $\leq-100 \mathrm{nT}$ ). They also reported that the possibility of SIR-ICME interaction structures causing geomagnetic storms is significantly higher than that of isolated SIRs or isolated ICMEs.

\subsubsection{Solar cycle variation of large SEP events}

As of December 2019, there were 46 large SEP events (> $10 \mathrm{MeV}$ proton intensity $\geq 10 \mathrm{pfu}$ ) in cycle 24 compared to 102 in cycle 23 (see Fig. 4c). This is a $55 \%$ reduction, more than the reduction in the SSN. Considering the period up to the middle of the maximum phase, the reduction reported previously was smaller ( 26\%, Gopalswamy et al. 2015c), most likely due to the lower Alfven speed in the rising phase of cycle 24. If we consider the highest energy particle events, viz., the ground level enhancement (GLE) events, the reduction is very drastic: there were 16 GLE events in cycle 23 compared to just 2 in cycle 24 , amounting to a reduction of $88 \%$. All large SEP events are associated with DH Type II bursts because the same CME-driven shock accelerates electrons (producing type II bursts) and ions (observed in space as SEP events). CMEs associated with SEP events and DH type II bursts are typically fast and wide (Gopalswamy et al. 2019). Not all DH type II bursts are associated with SEP events because of connectivity issues, high particle background, and $<10$ pfu events. The number of DH type II bursts decreased from 339 in cycle 23 to 181 in cycle 24 (see Fig. 6d), indicating a decline by $47 \%$, very similar to the reduction in FW CMEs (50\%) because most of the type II bursts are due to FW CMEs.

\subsubsection{Summary of solar cycle variation}

Table 2 summarizes the Earth-affecting events in solar cycles 23 and 24 in comparison with the SSN. In addition to SSN, we have shown major soft X-ray flares, regular CMEs, fast and wide CMEs, halo CMEs, and ICMEs followed by the heliospheric consequences (DH type II bursts, large SEP events, and major geomagnetic storms). The decline in solar activity in cycle 24 is 
Table 2 Earth-affecting events in solar cycles 23 and 24 in comparison with the SSN

\begin{tabular}{|c|c|c|c|c|}
\hline Property & Cycle 23 & Cycle 24 & Change & Change/SSN \\
\hline SSN & 81 & 49 & $-39 \%$ & $0 \%$ \\
\hline Major flares (M\&X) & 1584 & 809 & $-49 \%$ & $-16 \%$ \\
\hline X-class flares & 128 & 49 & $-62 \%$ & $-37 \%$ \\
\hline M-class flares & 1456 & 760 & $-48 \%$ & $-14 \%$ \\
\hline All CMEs (width $\left.\geq 30^{\circ}\right)^{\mathrm{a}}$ & $8429^{c}$ & 8470 & $+1 \%$ & $+66 \%$ \\
\hline Fast \& wide $\mathrm{CMEs}^{\mathrm{a}}$ & $501^{c}$ & 253 & $-50 \%$ & $-17 \%$ \\
\hline Halo CMEs ${ }^{a}$ & $409^{c}$ & 323 & $-21 \%$ & $+30 \%$ \\
\hline ICMEs ${ }^{b}$ & 307 & 208 & $-32 \%$ & $+12 \%$ \\
\hline Magnetic clouds (MC) & 114 & 86 & $-25 \%$ & $+25 \%$ \\
\hline Ejecta (EJ) & 193 & 122 & $-37 \%$ & $+4 \%$ \\
\hline DH Type II bursts & 339 & 181 & $-47 \%$ & $-12 \%$ \\
\hline Large SEP events & 102 & 46 & $-55 \%$ & $-26 \%$ \\
\hline GLE events & 16 & 2 & $-88 \%$ & $-79 \%$ \\
\hline Magnetic storms (Dst $<-100 \mathrm{nT}$ ) & 86 & 22 & $-74 \%$ & $-58 \%$ \\
\hline CIR storms & 11 & 2 & $-82 \%$ & $-70 \%$ \\
\hline CME storms & 75 & 20 & $-73 \%$ & $-56 \%$ \\
\hline
\end{tabular}

${ }^{a}$ From the search engine in the CDAW catalog (http://www.Imsal.com/solarsoft/www_getcme_list.html)

${ }^{\mathrm{b}}$ From http://www.srl.caltech.edu/ACE/ASC/DATA/level3/icmetable2.htm

Includes 223 Width $\geq 30^{\circ} \mathrm{CMEs}, 16 \mathrm{FW}$ CMEs, and 13 halo CMEs estimated to have occurred during the 4-month SOHO/LASCO data gap

represented by the 39\% drop in the cycle-averaged SSN. Most events declined more than the SSN did in cycle 24, except for ICMEs (declined by 32\%), halo CMEs (declined by $21 \%$ ), and the general population $\left(\mathrm{W} \geq 30^{\circ}\right.$ ) of CMEs (increased by $1 \%$ ). When the numbers are normalized to SSN, these three types of events show a clear increase in cycle 24. Fast and wide CMEs declined in cycle 24 by $\sim 47 \%$, slightly more than the SSN did. When normalized to SSN, the number of FW CMEs per SSN declined only by $17 \%$ in cycle 24 , somewhat similar to the decline in major soft X-ray flares and DH type II bursts. The general population of CMEs with width $>30^{\circ}$ clearly showed no decrease in number; when normalized to SSN, the average number of CMEs per SSN increased by $66 \%$. The two phenomena that showed the deepest decline are the major geomagnetic storms (74\%) and GLE events (88\%). Both these events are related to the heliospheric magnetic field strength, which significantly declined in cycle 24 . The reduced heliospheric magnetic field results in the weaker heliospheric pressure leading to the anomalous expansion of CMEs and the attendant magnetic dilution in CMEs. In the case of SEP events, the particle acceleration efficiency depends on the strength of the heliospheric magnetic field, hence a reduction in the latter results in less efficient acceleration, and hence, particles do not attain high energies (Gopalswamy et al. 2014a, b). The reduction in the heliospheric magnetic field is also likely to be responsible for weaker CIR storms: the decreased MHD compression of the weaker field does not increase it to high levels.

\subsection{Summary}

To conclude this section, we provide a list of catalogs of Earth-affecting transient events that have been compiled and maintained online by many workers in the past (Table 3). The catalogs include lists of observed solar flares, CMEs, ICMEs in the inner heliosphere, ICMEs at in situ, interplanetary shocks, ICMEs with solar sources, SIRs, and SEPs. These event catalogs are useful resources for facilitating research for the wide community.

\section{Progress in theories of CMEs and ICMEs}

\subsection{Introduction}

It is generally accepted that CMEs are driven by the free magnetic energy stored in the non-potential magnetic fields. There is a general consensus that the erupted structure is twisted, where the most common magnetic structure employed in modeling is a flux rope, i.e., a cylindrical plasma structure with a magnetic field draped around the central axis (Lepping et al. 1990). The eruption of the twisted magnetic structure is interrelated with the magnetic reconnection of the surrounding coronal magnetic field, releasing both thermal and nonthermal energy and producing a number of effects (Priest and Forbes 2002). The magnetic dips of the flux rope can support cool plasma, in which case also an eruptive filament can be observed. The whole process is 
Table 3 Catalogs of Earth-affecting solar transients, including flares, CMEs, ICMEs-IH (inner heliosphere), ICMEs-IS (in situ), shocks, SIRs and SEPs

\begin{tabular}{|c|c|c|c|}
\hline Type & Acronym & Description and link & Reference(s) \\
\hline Flares & - & $\begin{array}{l}\text { Solarsoft latest events on solar flares } \\
\text { https://www.Imsal.com/solarsoft/latest_events/ }\end{array}$ & - \\
\hline CMEs & CDAW & $\begin{array}{l}\text { SOHO CME catalog } \\
\text { https://cdaw.gsfc.nasa.gov/CME_list/ }\end{array}$ & (Yashiro et al. 2004) \\
\hline CMEs & SEEDS & $\begin{array}{l}\text { SOHO and STEREO CME catalogs based on automated method. } \\
\text { http://spaceweather.gmu.edu/seeds/ }\end{array}$ & (Olmedo et al. 2008) \\
\hline CMEs & CACTUS & $\begin{array}{l}\text { SOHO and STEREO CME catalogs based on automated method } \\
\text { http://sidc.oma.be/cactus/ }\end{array}$ & (Robbrecht and Berghmans 2004) \\
\hline CMEs & ARTEMIS & $\begin{array}{l}\text { SOHO CME catalog based on automated method } \\
\text { http://cesam.lam.fr/lascomission/ARTEMIS/index.html }\end{array}$ & (Boursier et al. 2005) \\
\hline CMEs & CORIMP & $\begin{array}{l}\text { SOHO CME catalog based on automated method } \\
\text { http://alshamess.ffa.hawaii.edu/CORIMP/ }\end{array}$ & (Byrne et al. 2012) \\
\hline CMEs & - & $\begin{array}{l}\text { STEREO COR1 catalog, including CMEs and other events } \\
\text { https://cor1.gsfc.nasa.gov/catalog/ }\end{array}$ & - \\
\hline CMEs & MVCC & $\begin{array}{l}\text { STEREO Dual-viewpoint CME catalog } \\
\text { http://solar.jhuapl.edu/Data-Products/COR-CME-Catalog.php }\end{array}$ & (Angelos Vourlidas et al. 2017) \\
\hline CMEs & KINCAT & $\begin{array}{l}\text { STEREO COR2 CMEs (2007-2013) with GCS model results } \\
\text { http://www.affects-fp7.eu/cme-database/index.php }\end{array}$ & (Bosman et al. 2012) \\
\hline ICMEs-IH & HELCATS & $\begin{array}{l}\text { STEREO HI event catalogs including HICAT, HIJoinCAT, HIGeoCAT } \\
\text { http://www.helcats-fp7.eu/ }\end{array}$ & (Harrison et al. 2018) \\
\hline ICMES-IS & - & $\begin{array}{l}\text { ACE ICMEs since } 1996 \text { complied by Richardson \& Cane } \\
\text { http://www.srl.caltech.edu/ACE/ASC/DATA/level3/icmetable2.htm }\end{array}$ & (Richardson and Cane 2010) \\
\hline ICMEs-IS & - & $\begin{array}{l}\text { WIND ICME catalog (1995-2015) } \\
\text { https://wind.nasa.gov/ICME_catalog/ICME_catalog_viewer.php }\end{array}$ & (Nieves-Chinchilla et al. 2018a, b) \\
\hline ICMES-IS & - & $\begin{array}{l}\text { WIND Magnetic Cloud list (1995-2006) } \\
\text { https://wind.nasa.gov/mfi/mag_cloud_pub1.html }\end{array}$ & (Lepping and Wu 2007) \\
\hline ICMES-IS & - & $\begin{array}{l}\text { WIND ICME catalog (1995-2015) } \\
\text { http://space.ustc.edu.cn/dreams/wind_icmes/ }\end{array}$ & (Chi et al. 2016) \\
\hline ICMES-IS & - & $\begin{array}{l}\text { ICMEs and other large scale structures in solar wind } \\
\mathrm{ftp} / / \text { wwww.iki.rssi.ru/pub/omni/ }\end{array}$ & (Yermolaev et al. 2009) \\
\hline Shocks & - & $\begin{array}{l}\text { CfA Interplanetary Shock Database } \\
\text { https://www.cfa.harvard.edu/shocks/ }\end{array}$ & - \\
\hline Shocks & - & $\begin{array}{l}\text { Heliospheric shock database at the University of Helsinki } \\
\text { http://ipshocks.fi/ }\end{array}$ & (Kilpua et al. 2015) \\
\hline ICMEs-CMES & - & $\begin{array}{l}\text { ICMEs and their solar sources in solar cycle } 24 \text { from GMU } \\
\text { http://solar.gmu.edu/heliophysics/index.php/GMU_CME/ICME_List }\end{array}$ & (Hess and Zhang 2017) \\
\hline Coronal Holes & - & $\begin{array}{l}\text { Coronal holes during SDO era list } \\
\text { https://cdsarc.unistra.fr/viz-bin/cat/J/other/SoPh/294.144 }\end{array}$ & (Heinemann et al. 2019b) \\
\hline SIRs & - & $\begin{array}{l}\text { STEREO SIR list } \\
\text { http://www-ssc.igpp.ucla.edu/forms/stereo/stereo_level_3.html }\end{array}$ & (Jian et al. 2019) \\
\hline SEPS & - & $\begin{array}{l}\text { Solar Proton Events from SWPC } \\
\text { https://umbra.nascom.nasa.gov/SEP/ }\end{array}$ & - \\
\hline SEPS & - & $\begin{array}{l}>25 \mathrm{MeV} \text { Proton Events Observed by the High Energy Telescopes on } \\
\text { the STEREO A and B Spacecraft and/or at Earth }\end{array}$ & (Richardson et al. 2014) \\
\hline SEPS & - & Catalog of 55-80 MeV solar proton events extending through solar cycles 23 and 24 & (Paassilta et al. 2017) \\
\hline SEPS & - & $\begin{array}{l}\text { STEREO/SEPT Solar Energetic Electron Event List } \\
\text { http://www2.physik.uni-kiel.de/stereo/downloads/sept_electron_events.pdf }\end{array}$ & (Nina Dresing et al. 2020) \\
\hline
\end{tabular}

known as "the standard flare-CME model" and is sketched in the left panel of Fig. 9. In "the standard magnetic cloud model," the erupting flux rope propagates away from the Sun, expanding at the same time, but stays attached to the Sun, i.e., remains a closed structure, as shown in the right panel of Fig. 9.

In order to understand the full picture of the CMEs, i.e., the magnetic structure of CMEs and processes 


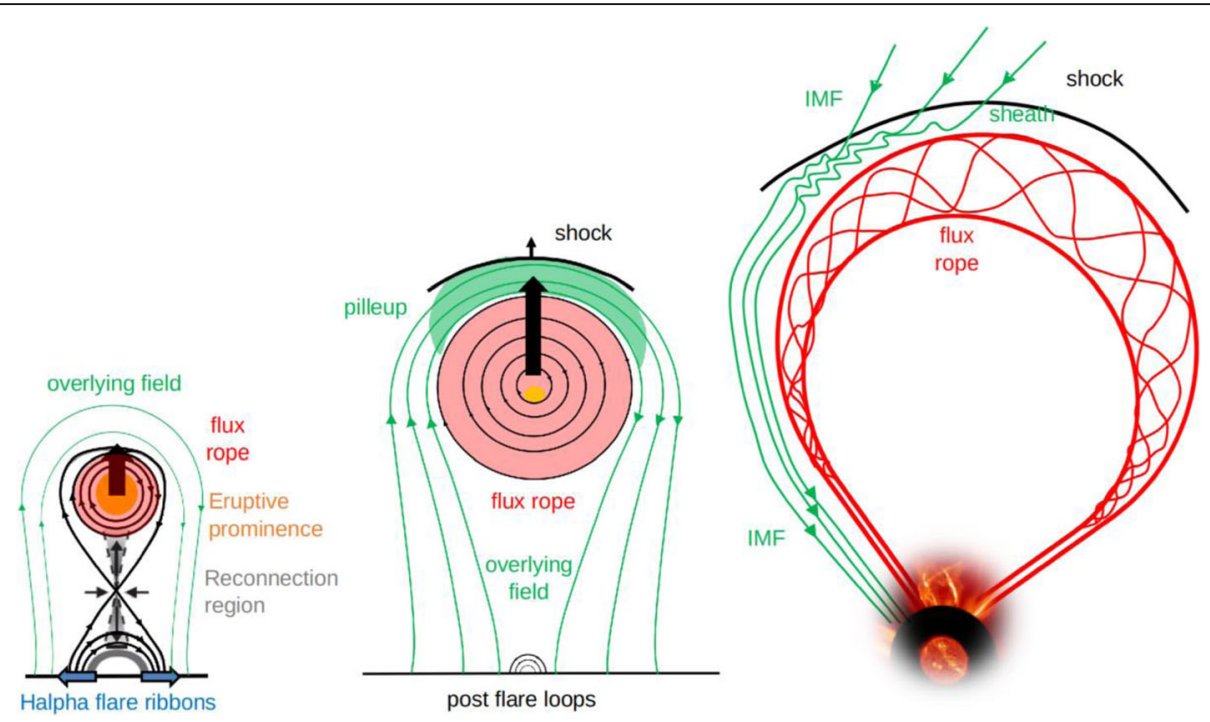

Fig. 9 Cartoon describing three different stages of CME evolution based on "the standard CME-flare model" and "the standard magnetic cloud model": the onset (left), post-eruption phase (middle), and interplanetary propagation (right)

involved in its initiation, evolution and propagation, we need theories to explain the observed properties of CMEs. Both numerical and analytical models need to be employed to reach deeper understanding of the origin and evolution of CMEs and provide a theoretical basis to the CME/ICME observations. The numerical modeling will be addressed in Section 4, while this section is devoted to analytical models and divided into subsections related to CME initiation, propagation, forward modeling, fitting to in situ measurements and finally interactions with other magnetic structures.

\subsection{Mechanisms, processes, and forces governing the CME take-off}

Comprehensive theoretical work on solar eruptive events started in the 1970s and early 1980s of the past century (Green et al. 2018). Many models were focused on the flux-rope configurations and their instabilities (see references in Chen 1989; Vršnak 1990), identifying Lorentz force as the main driving element of the eruption, and emphasizing the importance of the amount of the magnetic field twist. For example, using the analytical approach, Chen (1989) and Vršnak (1990), independently considered in their semi-toroidal flux-rope models a number of effects that were before taken only partially or were neglected. The flux-rope models progressively developed, becoming more sophisticated. A particular important step was the analytical model presented by Titov and Demoulin (1999), which took into account relevant effects and provides an idealized, but quite realistic configuration of a semi-toroidal flux rope embedded in a coronal arcade. This model was later on included in a number of analytical studies, or numerical simulations of the loss of equilibrium in general, and in particular, in studies of kink and torus instabilities (Török and Kliem 2003, 2005; Török et al. 2004; Török et al. 2010; Kliem and Török 2006; Kliem et al. 2014).

More recently in the past decade, which includes also the VarSITI/ISEST era, there was significant progress in physical understanding of solar eruptions. A number of papers were published considering the pre-eruptive storage of free energy and helicity, processes that cause the evolution of the system towards an unstable state, criteria defining stable/unstable configurations, initiation of the eruption, the dynamics of the eruption itself, as well as the analysis of the effects of the eruption in the ambient corona (see recent reviews by Aulanier 2013; Schmieder et al. 2015; Green et al. 2018; Patsourakos et al. 2020). Although it was clearly demonstrated that the active region magnetic complexity and its dynamics, free-energy, and helicity content are essential parameters, a number of open questions appeared. All of them can be summarized in the fact that we still do not understand why active regions that are quite similar according to the mentioned basic characteristics sometimes produce an eruption and sometimes not. Obviously, some key parameters are still missing, and consequently, this makes the eruption forecasting still highly unreliable.

The aforementioned standard CME-flare model was realized and investigated in detail in 3D using a zero- $\beta$ MHD simulation of an initially potential bipolar field, which evolves by means of line-tied shearing motions in the photosphere (Aulanier et al. 2010; Aulanier et al. 2012). This 3D standard model has been exploited to investigate the properties of $3 \mathrm{D}$ slip-running reconnection 
in eruption regions (Janvier et al. 2013a, b), evolution of localized currents in flare ribbons (Janvier et al. 2014; Barczynski et al. 2020), vortex and sink flows related to coronal implosion (Zuccarello et al. 2017), and downward Lorentz force in the Sun's photosphere during eruptions (Barczynski et al. 2019). (2018) made a review on the electric currents in the pre-eruption state and in the course of the eruption of solar magnetic structures.

Apart from general theoretical, numerical, or observational considerations regarding the mentioned preeruption configurations, a number of papers were published being focused more on specific processes that lead to the eruption than to general properties of active regions. For example, Török et al. (2013) analyzed in detail the consequences of rotational motions at the footpoints of a flux rope in one well-observed event. The analysis provided a very detailed physical interpretation of characteristics of the pre-eruptive evolution of the magnetic configuration, the initiation of the eruption, the dynamics of the eruption, as well as the evolution of an ambient magnetic system. On the other hand, Vršnak (2019) considered the rotating flux-rope leg process in more general terms and showed that except in quite extreme cases, such as considered by Török et al. (2013), the twisting motion does not allow for poloidal flux injection sufficient to explain the observed speeds of the slow rise of the flux rope in the gradual pre-eruptive stage. On the other hand, it was shown that the emerging flux process is viable to cause rising speed close to that observed in the pre-eruptive phase. Let us note that both processes eventually lead to a loss of equilibrium of the system if persistent long enough (Green et al. 2018).

In the last decade, special attention was paid to the role of the reconnection below the erupting flux rope in the dynamics of the eruption, primarily based on a number of observational results directly relating the eruption-related flare energy release and the eruption acceleration. This type of reconnection has three very important consequences. First, it reduces the tension of the overlying field, which tries to prevent the eruption and, in some cases, can cause a failed eruption. Second, it supplies the flux rope with a fresh poloidal flux that has a twofold effect. One is a direct enhancement of the upward-directed component of the hoop-force. The other is weakening of the self-inductive effect of the expanding structure that should lead to fast electriccurrent decrease, and thus a weakening of the Lorentz force. In this respect, it is worth mentioning the paper by Vršnak et al. (2016), where it was clearly demonstrated that the peak acceleration of the flux rope is dependent on the reconnection rate and the peak velocity is proportional to the total reconnected flux. Moreover, it was shown that the flux-rope acceleration time profile, as well as the velocity time profile, are closely synchronized with the time evolution of the reconnection rate. These theoretical results are able to fully explain the effects observed in the CME-flare relationship, as discussed in Section 2.4.

\subsection{CME propagation from corona through interplanetary space}

The kinematical evolution of CMEs can be divided into three phases: (1) slow rising phase during the CME initiation; (2) impulsive or main acceleration phase; (3) interplanetary propagation phase (Zhang and Dere 2006; Temmer 2016). There are three competing forces governing CME kinematical evolution, the gravitational force, Lorentz force and the MHD drag.

The Lorentz force mainly provided by the magnetic field in the corona is introduced in Section 3.2, while the MHD drag can be well represented by the aerodynamic drag equation and is believed to be dominant in the interplanetary propagation phase (Cargill 2004; Vršnak and Žic 2007). The latter is supported by observations showing deceleration of fast CMEs and acceleration of slow CMEs (Sheeley et al. 1999; Gopalswamy et al. 2020; Sachdeva et al. 2015). This concept was previously introduced into a simple Empirical Shock Arrival (ESA) model (Gopalswamy et al. 2001a, b), Expansion Speed Model (ESM) (Schwenn et al. 2005), and more recently in an Effective Acceleration Model (EAM) (Paouris and Mavromichalaki 2017), an analytical observation-driven model by (Liu et al. 2017a) and an empirically driven piston shock model by (Corona-Romero et al. 2017). Based on the concept of CME propagation being governed by MHD drag, an analytical drag-based model (DBM) was introduced by (Vršnak et al. 2013). DBM was found to very successfully describe heliospheric propagation of CMEs (Vršnak et al. 2014; Hess and Zhang 2014), therefore it was expanded to different geometries in recent years: most notably using a 2DCone geometry (Žic et al. 2015) and 2D ellipse front (Möstl et al. 2015). Note that the interplanetary acceleration obtained by Gopalswamy et al. (2001a, b) is proportional to CME speed and hence related to the Stokes drag, as opposed to the aerodynamic drag proportional to the square of the CME speed.

Observations by STEREO-HI instrument lent support to empirical models. The elongation conversion models were previously typically combined with fitting algorithms to determine CME arrival, assuming constant speed, as discussed in Section 2.2.4. However, the constant speed assumption was recently substituted with DBM and combined with a newly developed Ellipse Conversion method for the HI observations into the ElEvoHI model (Rollett et al. 2016). 
CMEs typically propagate radially, although deviations were found, namely rotations (Lynch et al. 2009; Vourlidas et al. 2011) and deflections (Wang et al. 2004; Lugaz et al. 2010b; Isavnin et al. 2014). Deflections by coronal holes have been previously modeled empirically, where a deflection from the original direction by a certain angle was quantified by the so-called coronal hole influence parameter (CHIP), assuming that CME continues to propagate radially (Gopalswamy et al., 2009; Mohamed et al. 2012). Interplanetary deflections were regarded previously as well, in a kinematic model assuming interplanetary spiral magnetic field and the background solar wind. As a result, CMEs faster than background solar wind are deflected to the east, whereas CMEs slower than background solar wind are deflected to the west (Wang et al. 2004, 2006). Recently, both deflections and rotations were included in a $3 \mathrm{D}$ CME propagation model, where the CME flux rope is represented with a rigid, un-deformable torus and deflections and rotations are calculated using the magnetic tension and magnetic pressure gradients calculated from magnetometer input (Kay et al. 2013, 2020b; Kay and Opher 2015).

Analytical models are easy to run and are not time consuming and therefore ideal for ensemble modeling (for details on ensemble modeling see Section 4). Therefore, it is not surprising that several recently developed analytical models have their ensemble version (see Table 4). Finally, recently a novel approach was adapted in semi-empirical CME propagation modeling, machine learning (Sudar et al. 2016; Liu et al. 2018d), which in the future might prove to be a powerful predictive tool.

\subsection{Forward modeling of CMEs}

Recently, there has been quite a development in the forward modeling of the magnetic structures using physicsbased empirical models. The forward models typically assume a specific morphology of a magnetic structure, i.e., flux rope, and evolve it, assuming specific propagation and expansion. For instance, Wood et al. (2017) use the croissant-like morphology described by Wood and Howard (2009), apply self-similar expansion and propagate it to Earth using either the Harmonic mean or Fixed Phi method as a kinematic model. With this method, it is possible to obtain a global shape of the structure, as well as its local size and orientation.

A step further is to include a specific flux rope magnetic field topology. Möstl et al. (2018) in their 3Dimensional COronal Rope Ejection (3DCORE) model used a tapered torus geometry and a Gold-Hoyle magnetic field topology (Gold and Hoyle 1960) and evolve it using DBM and self-similar expansion. They constrain the magnetic field values using measurements at Mercury. In their Flux Rope from Eruption Data (FRED) method, Gopalswamy et al. (2018c) evolve self-similarly a croissant shaped flux rope obtained by the Graduated Cylindrical Shell (GCS) model and assume a Lundquisttype magnetic field topology (Lundquist 1951) constrained using reconnection flux computed from the area under post-eruption arcades (Gopalswamy et al. 2017b; Pal et al. 2018; Sarkar et al. 2020). It should be noted that a similar approach was studied previously by Savani et al. (2015) and Temmer et al. (2017), where several options were regarded to constrain the magnetic field (reconnection flux computed from $\mathrm{H}$-alpha ribbons, dimming flux, and non-linear force-free modeling). Kay et al. $(2017,2020 \mathrm{~b})$ also use a Lundquist-type magnetic structure along with the shape and size represented with a rigid, un-deformable torus, which is the output of their ForeCAT model. Their ForeCAT In situ Data Observer (FIDO) model primarily assumes self-similar expansion and constant time-shift; however, with the addition of some free parameters, they are able to also simulate distortion, i.e., pancaking. They also extensively study the sensitivity of the model using ensembles (Kay and Gopalswamy 2018), although in its current form the magnetic structure is free-fitted to the in situ measurements. Recently, the FIDO model is improved by incorporating the forward model of the CME-driven shock and sheath, as FIDO-SIT (Sheath Induced by Transient) (Kay et al. 2020a, b). Flux rope in the 3D (FRi3D) model by Isavnin (2016) uses a croissant-like shape, but allows it to deform while expanding due to front flattening, pancaking, and skewing. The assumed magnetic structure is of Gold-Hoyle type and the whole FR is free-fitted to the in situ measurements. Finally, probably the most extensive forward modeling study was performed by Patsourakos et al. (2016), who used GCS croissant for the CME shape, propagated it using DBM, and then applied several different combinations for estimating the magnetic structure topology (including non-force free magnetic structures by Hidalgo et al. 2000 and Cid et al. 2002), as well as the initial helicity and expansion.

Although most of the forward modeling procedures are focused on reproducing the magnetic structure at a certain heliospheric distance, other applications might be noteworthy as well. A similar procedure was recently adopted in the Forbush decrease model (ForbMod) by (Dumbović et al. 2018a, b), who expanded the GCS croissant using empirical power-law relations for size and magnetic field, assuming constant-speed propagation and homogeneous magnetic field in order to derive galactic cosmic ray counts. The evolution of the internal properties (e.g., the plasma temperature, density, velocity, and heating) of CMEs is also one of the important research aspects, but it is limited to a certain position or a certain time by using remote sensing and in situ observations (e.g., Wang et al. 2005; Liu et al. 2006; Bemporad 
Table 4 List of recent analytical and semi-empirical models of CMEs/ICMEs

\begin{tabular}{|c|c|c|c|}
\hline Type & Acronym & Short description & Reference \\
\hline$P$ & EAM & Empirical; shock propagation & Paouris and Mavromichalaki (2017) \\
\hline$P$ & - & Empirical; shock propagation & Liu et al. (2017b) \\
\hline$P$ & - & Empirical; shock propagation & Corona-Romero et al. (2017) \\
\hline$P$ & ElEvo & DBM+2D ellipse; shock propagation & Möstl et al. (2015) \\
\hline$P$ & $\begin{array}{l}\text { DBM/ } \\
\text { DBEM }\end{array}$ & $\mathrm{DBM}+2 \mathrm{D}$ Cone; CME propagation & $\begin{array}{l}\text { (Žic et al. 2015) } \\
\text { (Dumbović et al. 2018a, b) (ensemble) }\end{array}$ \\
\hline$P$ & PDBM & 1D DBM; probabilistic; CME propagation & (Napoletano et al. 2018) (ensemble) \\
\hline$P$ & ElEvoHI & $\mathrm{DBM}+\mathrm{EIEvo}+\mathrm{HI}$ fitting; shock propagation & $\begin{array}{l}\text { Rollett et al. } 2016 \text { (Amerstorfer et al. 2018) } \\
\text { (ensemble) }\end{array}$ \\
\hline $\mathrm{P}$ & ForeCAT & CME propagation; deflection; rotation & $\begin{array}{l}\text { (Kay et al. 2013) } \\
\text { (Kay and Opher 2015) }\end{array}$ \\
\hline$P$ & - & Machine learning; CME propagation & (Sudar et al. 2016) \\
\hline$P$ & $\begin{array}{l}\text { CAT- } \\
\text { PUMA }\end{array}$ & Machine learning, shock propagation & Liu et al. 2018a \\
\hline$P$ & - & Oblique collision in 3D + constrain conservation of momentum & $\begin{array}{l}\text { Mishra et al. (2016) } \\
\text { Mishra et al. (2017) }\end{array}$ \\
\hline EC & - & triangulation & Liu et al. (2017a) \\
\hline FM & - & $\begin{array}{l}\text { Croissant + self-similar expansion + HM/fixed Phi } \\
\geq \text { size, orientation }\end{array}$ & Wood et al. (2017) \\
\hline FM & 3DCORE & Torus + self-similar expansion + DBM + Gold-Hoyle $\geq$ magnetic structure & Möstl et al. (2018) \\
\hline FM & FRED & GCS + self-similar expansion + Lundquist $\geq$ magnetic structure & Gopalswamy et al. (2017c) \\
\hline FM & FIDO & Torus + self-similar expansion + Lundquist $\geq$ magnetic structure & $\begin{array}{l}\text { Kay et al. 2017; Kay and Gopalswamy (2018) } \\
\text { (ensemble) }\end{array}$ \\
\hline FM & FIDO-SIT & FIDO + forward model of shock and sheath & Kay et al. (2020a) \\
\hline FM & FRi3D & Deformable croissant + Gold-Hoyle $\geq$ magnetic structure & Isavnin (2016) \\
\hline FM & - & $\begin{array}{l}\text { GCS + power-law expansion + DBM +(non) force free FR } \geq \text { magnetic } \\
\text { structure }\end{array}$ & (Patsourakos et al. 2016) \\
\hline FM & ForbMod & GCS + power-law expansion $\geq$ Forbush decrease & (Dumbović et al. 2018a, b) \\
\hline FM & FRIS & CME internal properties & Mishra and Wang 2018; Mishra et al. (2020) \\
\hline FIT & - & Force free + circular-cylindrical + velocity modified; & $\begin{array}{l}\text { Wang et al. (2015) } \\
\text { Wang et al. (2016d) }\end{array}$ \\
\hline FIT & - & Force free + circular-cylindrical $+\mathbf{B}$ modified & Lepping et al. (2018) \\
\hline FIT & - & Force free + circular-cylindrical + boundary pitch-angle treatments & Nishimura et al. (2019) \\
\hline FIT & - & Force free; circular-cylindrical + varying prescribed twist & Vandas and Romashets (2019) \\
\hline FIT & - & $\begin{array}{l}\text { Non-force free; torus + non-uniform cross-section; plasma pressure + proton } \\
\text { current density }\end{array}$ & $\begin{array}{l}\text { Hidalgo and Nieves-Chinchilla 2012; Hidalgo } \\
\text { (2014a, b) } \\
\text { Hidalgo (2016) }\end{array}$ \\
\hline FIT & - & Non-force free; force distribution + circular-cylindrical; elliptic-cylindrical & $\begin{array}{l}\text { Nieves-Chinchilla et al. 2016; Nieves-Chinchilla et al. } \\
(2018 a, b)\end{array}$ \\
\hline GS & - & GS reconstruction + toroidal geometry & Hu et al. (2017) \\
\hline
\end{tabular}

and Mancuso 2010; Susino et al. 2013; Susino and Bemporad 2016). In a novel approach, GCS is used to calculate the CME volume and density to derive the evolution of the magnetic ejecta and sheath density from Sun to Earth (M. Temmer et al. 2021). To continuously figure out the internal state of an individual CME during its heliospheric propagation, a self-similar flux rope internal state (FRIS) model was proposed by Wang, et al. (2009), providing the variations of the polytropic index of the
CME plasma, the average Lorentz force and the thermal pressure force inside a CME with heliocentric distance. Recently, Mishra and Wang (2018) improved the FRIS model, by constraining it with the observed propagation and expansion behavior of a CME and deriving a few additional parameters (absorbed heat, entropy, heating rate, and entropy changing rate). This model was then implemented to a slow (Mishra and Wang 2018) and fast (Mishra et al. 2020) CME respectively, which showed 
that during the propagation (1) the expansion was driven by the thermal force inside the CME but prevented by the Lorentz force, (2) the slow CME released heat before it reached an adiabatic state and then absorbed heat, and (3) the fast CME was in the heat-releasing state throughout its journey.

\subsection{In situ fitting of ICMEs}

ICMEs can be identified by a number of typical properties that differ from those of the ambient solar wind (Gosling 1990; Wimmer-Schweingruber et al. 2006; Zurbuchen and Richardson 2006; Rouillard 2011; Kilpua et al. 2017). Among ICMEs there is a subset, called magnetic clouds (MCs), which were first identified by Burlaga et al. (1981), and then studied widely in the past decades. MCs exhibit a smooth rotation of the magnetic field direction through a large angle, an enhanced magnetic field strength, and a low proton temperature (Burlaga et al. 1981). MCs play an important role in understanding the evolution of CMEs in the heliosphere and are one of the main drives of space weather events (Wilson 1987; Gopalswamy et al. 2008; Gopalswamy et al. 2015a, b, c; Tsurutani et al. 1988; Huttunen et al. 2002; Wu and Lepping 2002; Cane and Richardson 2003; Zhang et al. 2007; Hidalgo 2011; Li et al. 2018).

MCs are believed to have a magnetic flux rope structure with two ends rooting on the Sun (Burlaga et al. 1981; Larson et al. 1997; Janvier et al. 2013a, b). So far, the observations about MCs could only rely on the in situ data along the MC pass path. To reconstruct the global configuration of MCs in 2D or 3D space, a variety of techniques has been developed. Based on the idea of force-free $(\nabla \times \mathbf{B}=\alpha \mathbf{B})$ magnetic configuration of MCs (Goldstein 1983), the symmetric-cylindrical models, with linear (Burlaga 1988; Lepping et al. 1990; Lepping 2003) and non-linear (Farrugia et al. 1999) force-free fields, were proposed. Other models were also developed by adopting different assumptions, e.g., (1) the expanding model (Farrugia et al. 1993; Vandas et al. 2006), (2) the models with distorted cross section (Romashets and Vandas 2003; Vandas et al. 2006; Démoulin and Dasso 2009), and (3) the torus model (Romashets and Vandas 2003; Marubashi and Lepping 2007). Based on the observations of pressure gradients inside MCs, some nonforce-free models were proposed and then improved (Hidalgo 2002; Mulligan and Russell 2001; Hidalgo et al. 2002; Cid et al. 2002; Hidalgo 2003).

Nowadays, with our deeper understanding about MCs, several new models have emerged, which are still based on the well-developed description of (non-)force-free magnetic configuration, but can provide extra information about MCs.

\subsubsection{Force-free magnetic field}

The assumptions of the cross section and the symmetry along the $\mathrm{MC}$ axis are two keys in the models. Keeping the cylindrical symmetry and the circular cross section, Wang et al., (Wang et al. 2015; Wang et al. 2016a, b, c, d) developed the velocity-modified force-free flux rope models, which can provide the MC kinetic information by incorporating the linear propagating motion away from the Sun, the expanding, and the poloidal motion of the plasma inside MCs with respect to the MC axis. Recently, Lepping et al. (2018) improved their previous model (Lepping et al. 1990) by modifying the magnetic field magnitude based on a B-modification scheme presented by Lepping et al. (2017). This model improves the fitted B-profile, but is applicable for use with data originating only at/near $1 \mathrm{AU}$. The models of linear force-free field with different boundary pitch-angle treatments and of non-linear force-free field with varying prescribed twist were derived by Nishimura et al. and Vandas and Romashets, respectively. Discarding the assumption of locally straight MC axis, the non-cylindrical models were developed. For example, Owens et al. (2012) developed a curved flux rope (CFR) model, assuming a circular cross section but bending the axial field in a similar manner to a Parker spiral magnetic field. This allows the radius of curvature of the axis and the cross-sectional extent of the MC to vary along the length of the axis. To model irregularities in MCs, Romashets and Vandas (2013) added a local irregularity in the form of a compact toroid into a cylindrical linear force-free magnetic structure. Furthermore, MCs in toroidal geometry can also be constructed by the constantalpha force-free magnetic field with elliptical cross sections (Vandas and Romashets 2017a) or uniform-twist force-free field with circular cross sections (Vandas and Romashets 2017b).

\subsubsection{Non-force-free magnetic field}

Inherited from Hidalgo (2003), a much more complicated model was improved in series (Hidalgo and NievesChinchilla 2012; Hidalgo 2013). Hidalgo and NievesChinchilla described MC topology with torus geometry and a non-uniform cross section. They established an intrinsic coordinate system for that topology, and then analytically solved the Maxwell equations in terms of it. The model was tested by applying it to the observations of multiple spacecraft by Hidalgo (2013). The model was further improved with inclusion of the plasma pressure (Hidalgo) and the proton current density (Hidalgo). Extending the concept of Hidalgo et al. (2002), NievesChinchilla et al. (2016) presented a circular-cylindrical flux-rope model by introducing a general form for the 
radial dependence of the current density, which can give the information on the force distribution inside MCs. The circular cross section was later improved to an ellipse (new elliptic-cylindrical flux rope model, Nieves-Chinchilla et al. 2018b), which, for the first time, allows us to completely describe MCs by nonorthogonal geometry.

Different from the above introduced techniques, the Grad-Shafranov (GS) reconstruction technique (Hu and Sonnerup 2001, 2002) doesn't presume a specific magnetic structure or cross section of MCs. It assumes that an asymmetric-cylindrical $\mathrm{MC}$ is in an approximately magnetostatic equilibrium with an invariant direction, and uses the GS equation to recover the magnetic field as well as the plasma pressure. With this technique, the boundaries of the $\mathrm{MC}$ need not first be identified in the data. Recently, trying to approach a more real flux rope topology, the GS reconstruction of MCs in toroidal geometry with rotational symmetry was developed $\mathrm{Hu}$ 2017; Hu et al. 2017).

The development of all the introduced techniques provides invaluable tools for extracting information about the properties of MCs and leads to our understanding of the underlying physics of MCs. However, it was shown that assessing their accuracy based on in situ data is challenging. Different comparisons of the MC properties have been performed between: (1) MHD simulations and fitting techniques (Riley et al. 2004; Vandas et al. 2010; Al-Haddad et al. 2011, 2019) and (2) different fitting models (Al-Haddad et al. 2013; Démoulin et al. 2013; Hu et al. 2013; Vandas et al. 2015; Vandas and Romashets 2015; Lepping et al.; Nishimura et al. 2019). While these studies largely support the applicability of examined techniques and methods to gain insight into the $\mathrm{MC}$ structure, they also reveal their limitations as well as reliability issues, which should be tackled in the future.

\subsection{CME-CME interactions}

Erupting from the Sun, coronal mass ejections (CMEs) will interact with different structures. The interaction between a CME and the magnetic fields in the corona and interplanetary space, and the solar wind govern the propagation and evolution of the CME itself (see Section 3.3 and 3.4). Magnetic reconnection between CMEs and ambient solar wind can lead to the peeling-off of the magnetic field lines and the erosion of the axial magnetic flux from the CMEs (Dasso et al. 2006; Gosling 2012; Ruffenach et al. 2012, 2015; Manchester et al. 2014; Lavraud et al. 2014; Wang et al. 2018). Furthermore, CMEs were found to be interacting with other CMEs by many observations (e.g., Gopalswamy et al. 2001a; Lugaz et al. 2012; Shen et al. 2012; Harrison et al. 2012; Liu et al. 2012; Liu et al. 2014; Oliveros et al. 2012; Möstl et al. 2012; Temmer et al. 2012, 2014; Webb et al.
2013; Shanmugaraju et al. 2014; Colaninno and Vourlidas 2015; Mishra et al. 2014, 2015, 2017), involving complicated physical processes, resulting in the changes of CME properties, forming complex structures, and playing an important role in leading to large solar energetic particle events (see Section 6) and intense geomagnetic storms. CME-CME interaction can result in the changes in CME speed, propagation direction (Xiong et al. 2009; Lugaz et al. 2012; Shen et al. 2012; Mishra et al.), radial extent as well as internal magnetic strength (Schmidt and Cargill 2004; Xiong et al. 2006; Lugaz et al. 2005, 2012, 2013). The speed change, or more physical, the natures of the collision of one CME by another, were widely studied, namely, inelastic (Lugaz et al. 2012; Maričić et al. 2004; Mishra et al. 2015) vs. elastic (Mishra et al. 2014, 2015) vs. super-elastic collision (Shen et al. 2012; Shen et al. 2013a, b, c; Colaninno and Vourlidas 2015). Note that the term of collision used here refers to that the main bodies of two CMEs are touching.

In determining the nature of collisions, most of the earlier studies have used a simplistic approach that CMEs are propagating exactly in the same direction, i.e., 1D head-on collision (with momentum conservation constraint, Mishra and Srivastava 2014). However, a relatively more precise analysis should be performed in 3D space. For the first time, Shen et al. (2012) studied an oblique collision of CMEs in 3D using imaging observations (Fig. 10a) and took several uncertainties into consideration, but did not constrain the conservation of momentum instead of indirectly evaluating it by analyzing the effect of the solar wind on the acceleration of the first CME. Recently, Mishra et al. addressed previous limitation and proposed a method for the 3D oblique collision (Fig. 10b), in which the post-collision directions and speeds are theoretically measured with different preset restitution coefficients, together satisfying the momentum conservation law, and the best-matched parameters with the observations are extracted. Based on this, Mishra et al. (2017) calculated the uncertainties in determining the nature of collisions quantitively, with different CME observed parameters considered, which then emphasized the possibility of a large uncertainty (see one example in Fig. 10c). Furthermore, Shen et al. (2017) presented four definitions of different types of collisions, i.e., a classical Newtonian definition, an energy definition, Poissons definition, and Stronges definition, helping with a deeper understanding of the determination of collisions in theory. They focused on the first two used in observational and numerical studies, and found out that these two definitions are not equivalent to each other when the colliding objects are expanding or contracting with a changing rate.

Including collision, CME-CME interaction could be involved in four forms: (1) the two-CME driven shock 


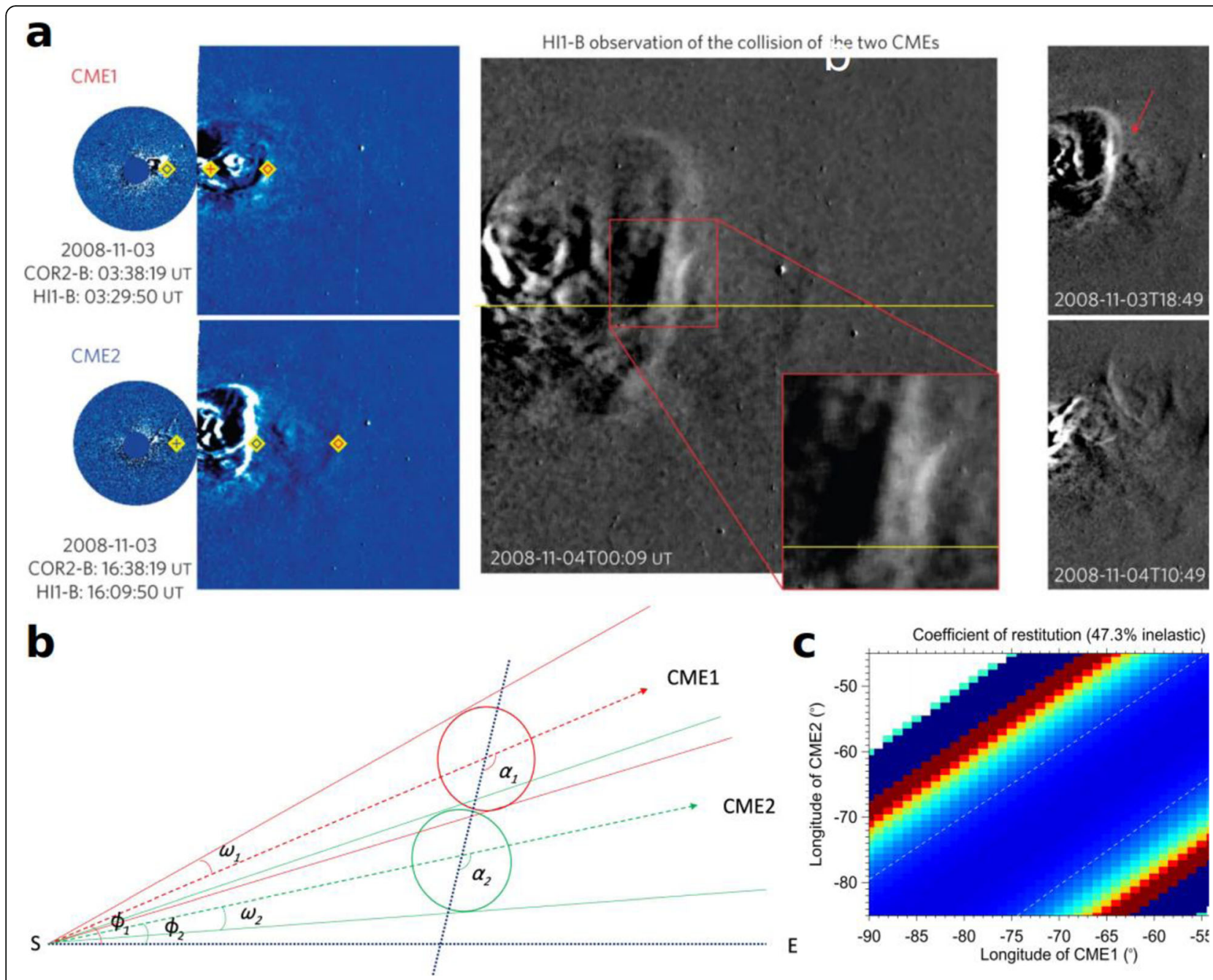

Fig. 10 a The super-elastic collision of two interacting CMEs observed in STEREO/HI (Shen et al. 2012). b Oblique collision of two CMEs assumed as spherical bubbles (Mishra et al.,). c The variation of the restitution coefficient with the uncertainties of pre-collision longitudes of CMEs considered (Mishra et al.,)

waves interact without the ejecta interacting, (2) one shock wave interacts with preceding magnetic ejecta, (3) the direct interaction between two ejecta, and (4) the reconnection between successive magnetic ejecta. The last three may result in a variety of complex structures at 1 AU: from 1) partial ongoing interaction of the preceding ejecta with a shock wave inside (Lugaz et al. 2015a, b; Wang 2003; Liu et al. 2018c; Wang et al. 2018; Xu et al. 2019), to full interaction of 2) the multiple magnetic cloud (MC) events (Wang et al. 2002; Wang 2003; Shen et al. 2011c; Lugaz et al. 2013), 3) a complex ejecta or compound stream (Burlaga et al. 2003), and 4) long-duration events (Dasso et al. 2009; Lugaz et al. 2013), leading to a hard understanding of the undisturbed conditions by in situ observations. Wang et al. (2018) proposed a recovery model based on Rankine-Hugoniot jump conditions to recover the shocked structure back to the uncompressed state in the first type of the interacted structure, which was later used to estimate the related interacting effects in causing geoeffectiveness (Shen et al. 2018a; Xu et al. 2019). The resulting structure from CME-CME interaction was discussed in detail in the review of Lugaz et al. (2017).

\subsection{Summary of recent analytical and semi-empirical models}

Table 4 shows the analytical and semi-empirical models of CMEs/ICMEs introduced above, which were proposed roughly during the VarSITI period (ca. 2014-2018). Models are listed according to their type: $\mathrm{P}=$ propagation, $\mathrm{EC}=$ elongation conversion, $\mathrm{FM}=$ forward modeling, FIT = in situ fitting, GS = Grad-Shafranov reconstruction. 


\section{Progress in numerical modeling of CMEs/ICMEs}

Magnetohydrodynamic (MHD) simulations have proved to be one of the most important tools to study the evolution of a coronal mass ejection (CME) in both corona and interplanetary space, and the modeled results can be used to analyze the initiation and propagation characteristics observed by ground-based and space-based instruments. Lugaz and Roussev (2011) gave a detailed review and discussion on the efforts to use numerical simulations of ICMEs to investigate the magnetic topology, density structure, energetics, and kinematics of ICMEs in the interplanetary space. In the book of Feng (2020), the author has provided a recent in-depth review of the field focusing primarily on the current status of MHD modeling for space weather with a thorough collection at the time of writing the book. Here, the review is devoted to recent progress of time-dependent MHD space weather modeling with the focus on such topics: the ambient solar wind, CME initiation and CME propagation, CME-solar-wind interaction, and CME-CME interaction, especially on the work performed in the second half of the 2010s.

\subsection{Modeling the background solar wind}

Numerical simulations have shown the importance of an accurate modeling of the background medium in which the disturbances propagate (Odstrčil and Pizzo 1999a, 1999b; Chané et al. 2005; Shen et al. 2007). In the past decade, due to the vast improvement in computational resources, the usage of 3D MHD models for reconstructing the solar corona and interplanetary solar wind has become almost routine. Moreover, based on much improved observations, it is possible to produce more realistic simulations, e.g., by the inclusion of the observational data through the boundary conditions or through data assimilation. Hayashi (2012) presented a treatment of observation-based time-dependent boundary conditions for the inner boundary sphere in the 3D MHD simulations of the global co-rotating solar wind structures. In order to adjust the model to the timevarying magnetic field on the bottom boundary, developed the model of the confined differential potential field (CDPF) to prescribe the bottom boundary condition, In addition, a modified version of this model was adopted as the module of the time-dependent 3D MHD simulation at the Joint Science Operation Center (JSOC) of SDO (Hayashi et al. 2015). The module could routinely generate $3 \mathrm{D}$ data of the time-relaxed minimum-energy state of the solar corona using the fulldisk magnetogram data from HMI/SDO.

In parallel, data assimilation has been included in the WSA model through ADAPT (Hickmann et al. 2015), which allows photospheric simulations to agree better with available observations from magnetograms. This model has been coupled to the 3D MHD LFM-Helio (Merkin et al. 2016a) to perform time-dependent simulations of the background solar wind. These simulations are able to reproduce more accurate details of smallscale of the heliospheric current sheet and corotating interaction regions.

Recently, by using magnetogram synoptic map images from GONG and theoretical/empirical models such as the PFSS model and WSA model, Shen et al. (2018a, b) applied a new boundary treatment to the 3D MHD simulation of solar wind and established the 3D IN (INterplanetary)-TVD-MHD model. The boundary conditions depend on five tunable parameters when simulating the solar wind for different phases of the solar cycle, and the simulated solar wind parameters are in good agreement with the observations most of the time. However, we know that models tend to fail when solar activity increases. The comparison of their modeled results with the in situ data throughout 2007 is shown in Fig. 11, which demonstrates that the simulation retrieves most of the high-speed streams (HSSs), and the duration time and the magnitude of the HSSs are largely consistent with those of the observations. Later, Yang and Shen (2019) presented a new method to construct the global distribution of solar wind parameters at the source surface using multiple observations and the ANN (Artificial Neural Network) technique, which could be used to provide a more realistic boundary condition for 3D MHD solar wind modeling.

By using CORHEL (CORona-HELiosphere), Linker et al. (2016) further developed a time-dependent study of the solar wind empirically driven by magnetic maps at a daily cadence using ADAPT. Their simulation showed both classic features of stream structure in the interplanetary medium often seen in steady-state models and evolutionary features unable to be captured in a steady-state approach. Their model results also compared reasonably well with 1 AU OMNI observations. As a rather mature space weather model, CORHEL is a coupled suite of models for simulating the solar corona and solar wind in 3D space, which provides three solar coronal models at present, including the Magnetohydrodynamic Algorithm outside a Sphere (MAS) polytropic model, MAS thermodynamic model, and the potential field source surface and Wang-Sheeley-Arge (PFSS-WSA) model. The heliospheric models involved in CORHEL are ENLIL (Odstrcil et al. 2004), the MAS-Heliosphere (MAS-H) models (Lionello et al. 2013), and the LFM-Helio (Merkin et al. 2016b).

Based on the SWMF (Space Weather Modeling Framework), van der Holst et al. (2014) developed the Alfvén wave solar model named AWSoM, which is a $3 \mathrm{D}$ MHD model that considers the anisotropy of ion temperature in the solar corona and the inner 

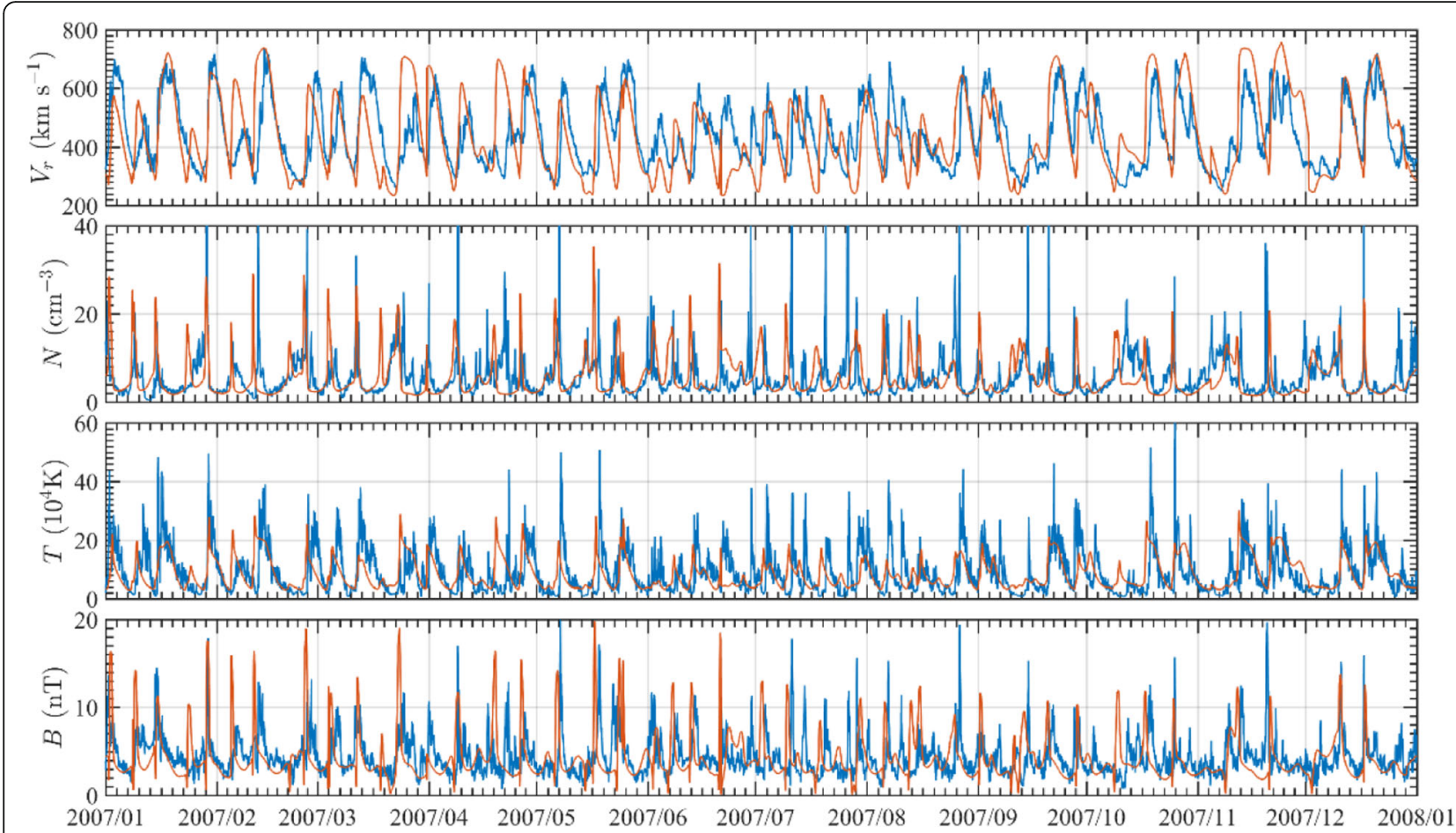

Fig. 11 Modeled (red lines) and observed (blue lines) time profiles of solar wind parameters at 1.0 au through all of 2007. From the top to the bottom, the panels show the speed $V_{r}$, number density $N$, temperature $T$, and total magnetic field strength $B$ (From Shen et al. 2018b). Besides the improvement in the treatment of the inner boundary condition, there have been significant improvements in other aspects of background solar wind simulations in recent years. Some of these are detailed below

heliosphere. In Meng et al. (2015), the AWSoM model has been applied to simulate the steady solar wind from the solar corona to 1 AU of CRs 2107 and 2123. Figure 12 a shows the simulated proton pressure anisotropy ratio and the simulated solar wind speed in the $Y=$ 0 and $Z=0$ planes for CR2107. The simulation results also show a reasonable agreement with in situ observations at $1 \mathrm{AU}$, as shown in Fig. 12b. Together with a coronal charge state evolution model, the Michigan Ionization Code (MIC, see Landi et al. 2012), Oran et al. (2015) employed the AWSoM model to calculate the elemental charge state evolution along the modeled open magnetic field lines. The charge state evolution model was initiated with the electron density, temperature, and speed simulated by the AWSoM wind model, and provided the first charge state calculation covering all latitudes in a realistic magnetic field. Evans et al. (2012) self-consistently coupled the Alfvén wave energy transport with the MHD equations. In this solar wind model, they introduced an additional dissipation mechanism: surface Alfvén wave (SAW) damping, which was weak in the polar regions, and strong in subpolar latitudes and the boundaries of open and closed magnetic fields. Their simulated results showed that SAW damping could reproduce regions of enhanced temperature at the boundaries of open and closed magnetic fields seen in both tomographic reconstructions in the low corona and Ulysses data in the heliosphere. Sokolov et al. (2013) presented a combined global model of the solar corona, the low corona, the transition region, and the top of the chromosphere. Their model used MHD Alfvén wave turbulence as the only momentum and energy source to heat the coronal plasma and drive the solar wind with different turbulence dissipation efficiencies in coronal holes and closed field regions. Recent developments include further validations of the AWSoM model (Gombosi et al. 2018; Sachdeva et al. 2019). It is known that the SWMF couples the models of Lower Corona (LC), Solar Corona (SC), Inner Heliosphere (IH), and other integrated components (Tóth et al. 2012). The models of SC, IH, and several other components are modeled by the BATS-R-US code. In the SC model, van der Holst et al. (2010) solved the two-temperature MHD equations with Alfvén wave heating and heat conduction on either Cartesian or spherical grid in a frame corotating with the Sun.

Feng et al. (2010) employed the SIP-CESE MHD model within a six-component overset grid for solar wind simulation. They numerically investigated the large-scale structures of interplanetary solar wind and the evolution of the heliospheric magnetic field. Feng et al. (2012) carried out the numerical studies for the solar 
(a)
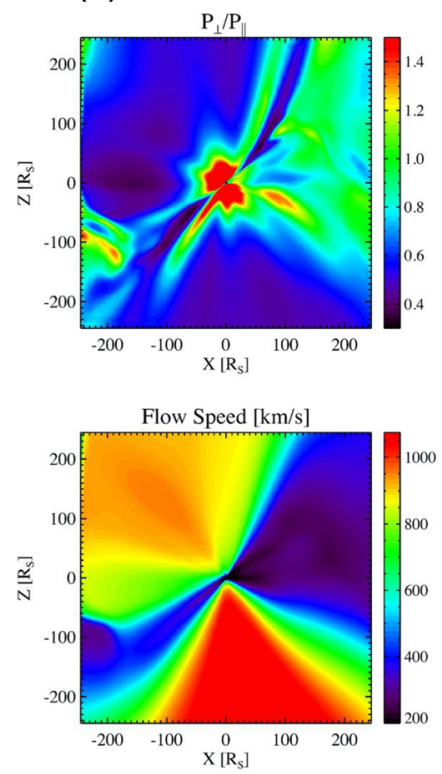
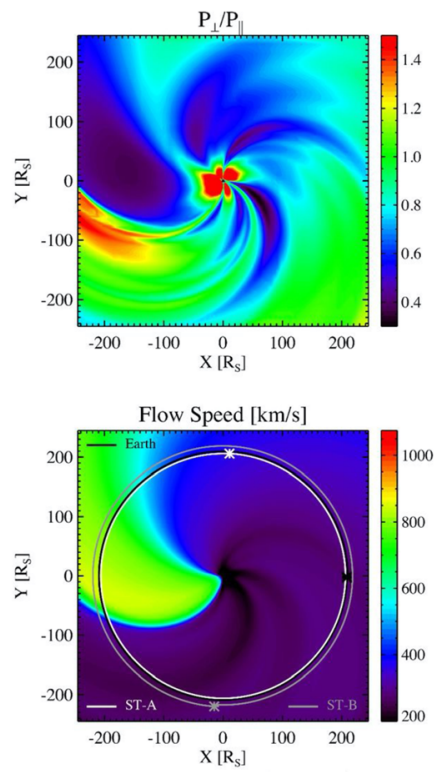

(b)
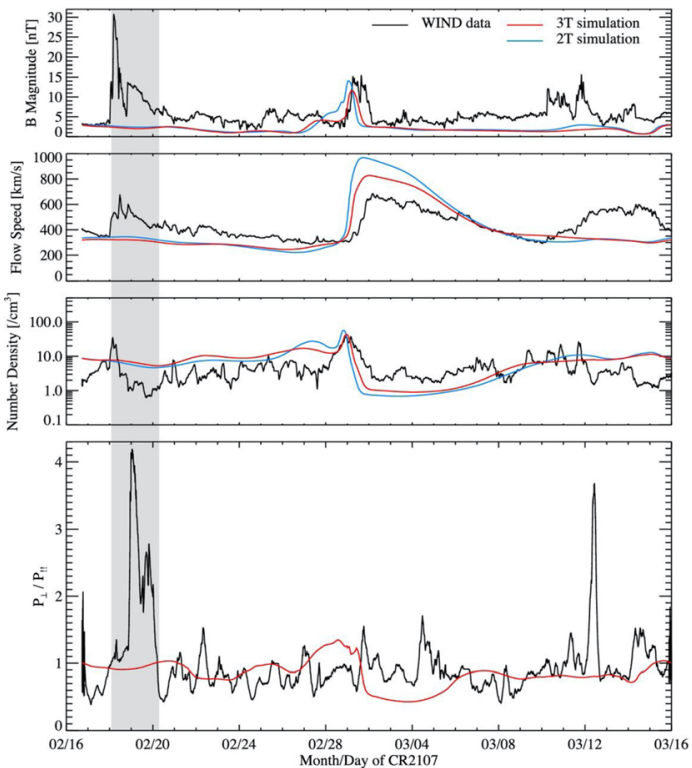

Fig. 12 a The simulated proton pressure anisotropy ratio $p_{\perp} / p_{-}$and the simulated solar wind speed in the $Y=0$ and $Z=0$ planes for $C R 2107$ by the AWSoM model, and the plot on the right bottom panel also shows the trajectories of the Earth, STEREO-A and B satellites projected to the $Z$ = 0 plane; $\mathbf{b}$ The simulated solar wind properties along the WIND orbit and the WIND data during CR2107 (From Meng et al. 2015)

wind background of different solar-activity phases by using the SIP-AMR-CESE MHD model, and their modeled results could reproduce many features near the Sun and in interplanetary space, e.g., the changing trends of the solar-wind parameters for the selected CRs, and the IMF polarities and their changes. Furthermore, Feng et al. (2015) investigated the solar wind evolution between the solar surface to the Earth's orbit from 1 July to 11 August 2008 with the SIP-AMR-CESE MHD model driven by the consecutive synoptic maps from GONG. Similarly, Li and Feng (2018) simulated the evolution of solar wind from the solar surface to the Earth's orbit during the year 2008, and evaluated simulated results quantitatively by comparison with in situ measurements.

Merkin et al. (2011) adapted the Lyon-FedderMobarry (LFM) model for the inner heliosphere, which was referred to as the LFM-helio model. They simulated the solar wind and heliospheric magnetic field between 0.1 and $2 \mathrm{AU}$ to study the disruption of a heliospheric current sheet fold during CR 1892 in the decline phase of solar cycle 22. (Merkin et al. 2016a) also presented a simulation study exploring heliospheric consequences of time-dependent changes at the Sun during a 2-month period in the beginning of the year 2008, in which they used the Air Force Data Assimilate Photospheric Flux Transport (ADAPT) model to obtain daily updated photospheric magnetograms and drive the WSA model of the corona. The results of the WSA model were used as a time-dependent boundary condition for the LFMhelio model. They compared the simulation results with ACE, STEREO-A and B near 1 AU, and MESSENGER spacecraft orbiting between 0.35 and $0.6 \mathrm{AU}$ and their simulations showed that time-dependent simulations could reproduce the gross-scale structure of the heliosphere.

The CRONOS model was employed to solve the equations of ideal MHD in a one-fluid model and to obtain realistic modeling solar wind conditions from $0.1 \mathrm{AU}$ to 2 AU (Wiengarten et al. 2013, 2014). Additionally, Wiengarten et al. (2015) incorporated turbulence transport into the Reynolds-averaged MHD equations in the framework of the CRONOS, which was used to investigate the effects on the turbulence evolution for transient events from $0.1 \mathrm{AU}$ to $1 \mathrm{AU}$ by injecting a CME from the inner boundary.

Shiota et al. (2014) developed the SUSANOO model (also see Shiota and Kataoka 2016), and they used it to simulate the ambient solar wind structure from 25 to 425 Rs covering 3 years (2007-2009). Their numerical results were in reasonable agreement with in situ measurements at Venus and Mars by Venus Express and Mars Express, respectively (Shiota et al. 2014).

The "European heliospheric forecasting information asset" (EUHFORIA) is another space weather forecasting model focus on the inner heliosphere, which is capable to provide MHD modeling of the ambient solar wind 
and the CME eruptions from 0.1 AU to $2 \mathrm{AU}$ (Moschou et al. 2017; Pomoell and Poedts 2018). EUHFORIA consists of an empirical coronal model and an MHD heliosphere model. The coronal model provides plasma and magnetic field parameters at $R_{b}=0.1 \mathrm{AU}$, which was then used as a boundary condition to drive the heliospheric model. The performance of the solar wind model was recently performed in the study of Hinterreiter et al. (2019).

Usmanov et al. (2018) presented a fully 3D MHD model of the solar corona and solar wind by coupling Reynolds-averaged solar wind equations with transport equations for turbulence energy, cross helicity, and correlation scale, from the coronal base to 5 AU. Their simulation results showed that the model could reproduce most of the solar wind parameters compared with Ulysses data during its first and third fast latitude transits.

Piantschitsch et al. (2017) developed a 2.5D MHD code to simulate the corona wave propagation and its interaction with a low-density region, such as the coronal hole $(\mathrm{CH})$. By using this new code, they also made a comprehensive analysis on the dependence on different Alfvén speeds inside the $\mathrm{CH}$ and initial amplitude of the incoming wave (Piantschitsch et al. 2018a, b). Their results depicted that the density value inside the $\mathrm{CH}$ influenced the phase speed and the amplitude values of density and magnetic field for all different secondary waves and there existed a correlation between the initial amplitude of the incoming wave and the amplitudes of the secondary waves as well as the peak values of the stationary features.

\subsection{Modeling CME initiation and propagation}

Jacobs and Poedts (2011) gave a detailed review about the state-of-the-art models for CME simulation before 2011. Here, we focus on the progress on the CME initiation, propagation, CME-CME interaction, and CMEsolar wind interaction, mainly after 2011, especially from years 2014 to 2019. The review by Manchester et al. (2017) includes a section about simulations and additional information about the main physical processes occurring during CME evolution in the inner heliosphere.

\subsubsection{Modeling CME initialization}

Most of the existing CME models are candidates for mimicking the morphology near the Sun, with the purpose of reproducing the plasma parameters comparable with $1 \mathrm{AU}$ observations, with the stated goal for many of them to move towards real-time space weather forecasting simulations. Presently, significant progress has been made towards improving the performance of the existing CME initialization models.
4.2.1.1 Cone model The cone model ((Fisher and Munro 1984; Zhao et al. 2002; Zhao et al. 2016a; Xie et al. 2004) is one of the popular CME initiation models because of its simplicity and relatively good match with CME arrival time observations. In most implementations of the model, the CME does not possess an internal magnetic field, but the input size, speed, and location are determined from coronal observations, typically from coronagraphs. In addition, due to its geometry and lack of internal magnetic field, the initiation does not include parameters related to CME orientation. By using cone model as CME initialization, Odstrcil et al. (2005) applied the 3D MHD simulation to the 12 May 1997 interplanetary event to analyze possible interactions of the ICME propagating in various steady-state and evolving configurations of the background solar wind.

Taktakishvili et al. (2011) reported the simulated results of selected well-observed halo CME events using a combined model of the WSA/ENLIL and the cone models. Their simulation results demonstrated that the combination of numerical models with the observations from coronagraph as input could give reasonably good results for the CMEs' arrival times for the selected set of "geoeffective" CME events. Bain et al. (2016) also combined the WSA-ENLIL and cone models to discuss shock connectivity in the August 2010 and July 2012 events. Dewey et al. (2015) integrated the cone model into the WSA-ENLIL model to study the CME-related solar wind perturbations on the Mercury system. Their simulation results demonstrated that the modeled results could be compared with the observations by the spacecraft of MErcury Surface, Space ENvironment, GEochemistry and Ranging (MESSENGER) during the period from March 2011 to December 2012.

Pomoell and Poedts (2018) integrated the cone model into the EUHRORIA model to simulate the CME events in the inner heliosphere during 17-29 July 2015. Also by combining the cone model and the EUHRORIA model, Scolini et al. (2018a, b) tested the effect of different CME shapes on the simulation results, and their simulation results showed that all the parameters specifying the CME shape in the model significantly affect simulation results at $1 \mathrm{AU}$ as well as the predicted CME geoeffectiveness.

4.2.1.2 Flux rope models Flux rope models have been shown to self-consistently reproduce many observed properties of CMEs, including the three-part density structure (Manchester et al. 2017). Contrary to the cone models, they include an internal magnetic field and may therefore reproduce not only the arrival time but also the magnetic field components when a CME impacts Earth. Due to their more complex nature, additional parameters are required to initialize such models, 
including the internal magnetic field strength or flux and the orientation of the flux rope. These models were first implemented in 3D MHD simulations by Roussev et al. (2003).

Lionello et al. (2013) improved the MAS-ENLIL model by inserting an out-of-equilibrium flux rope in the coronal model within 7 Rs as CME initiation model, and they simulated the propagation of an interplanetary CME (ICME) from 18 Rs to 1.1 AU. Their simulation results showed that the improved model could follow the propagation of the CME accurately. By using the MAS/ MAS- $\mathrm{H}$ model combined the modified Titov-Démoulin (TDm) model (Titov et al. 2014), Török et al. (2018) inserted a magnetically stable flux rope to generate a CME close to the observed properties of the 2000 July 14 "Bastille Day" eruption. The properties of the CME as it propagates were studied based on MHD simulations of solar eruptions from near the Sun to the Earth. Figure 13 a shows the initial flux-rope field lines, (b) depicts the field lines of the flux-rope core at $t=164.10$, shortly after eruption onset, and (c) and (d) demonstrates the interplanetary magnetic field and ICME flux rope at $t=256$, shortly before it reached $1 \mathrm{AU}$.

Using the Titov-Démoulin (TD) flux-rope model to initiate the CME, Jin et al. (2013) simulated a fast CME erupted from active region NOAA AR 11164 during CR2107. Simulations of this CME event were conducted with 1T (one-temperature) and 2T (two-temperature: coupled electron and proton) MHD models. The authors compared the propagation of this fast CME and the thermodynamics of CME-driven shocks in both the $1 \mathrm{~T}$ and $2 \mathrm{~T}$ CME simulations, and their results demonstrated the importance of the electron heat conduction in conjunction with proton shock heating in order to produce the physically correct CME structures and CME-driven shocks.

Based on the solar wind background constructed by the AWSoM SC model (van der Holst et al. 2014), Jin et al. (2016) presented a numerical simulation on the CME which occurred at 00:04 UT on 15 February 2011, which was initiated by using the analytical Gibson-Low (GL) flux rope model (Gibson and Low 1998) with different parameters. Their simulation results showed that a CME's impact on the surrounding solar wind structures would be influenced by the magnetic strength of these structures, their distance to the source region, and the interaction between the CME with the large-scale magnetic field. Jin et al. (2017a) developed a new data-driven tool called Eruptive Event Generator Gibson-Low (EEGGL) to automatically determine the GL flux rope parameters using synoptic magnetogram data from GONG and CME speed derived from the observations of SOHO/LASCO. By combining the EEGGL model and the AWSoM solar wind model, Jin et al. (2017a)

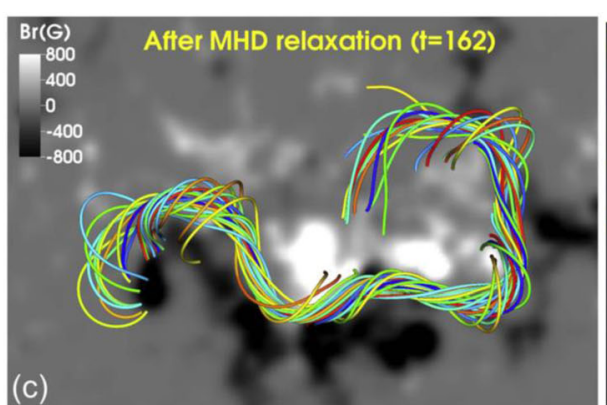

(a)

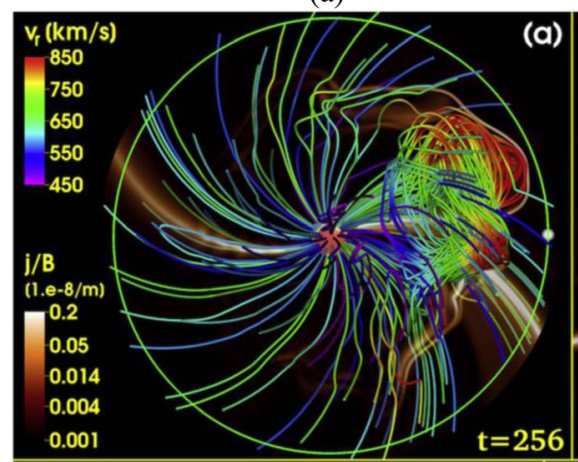

(c)

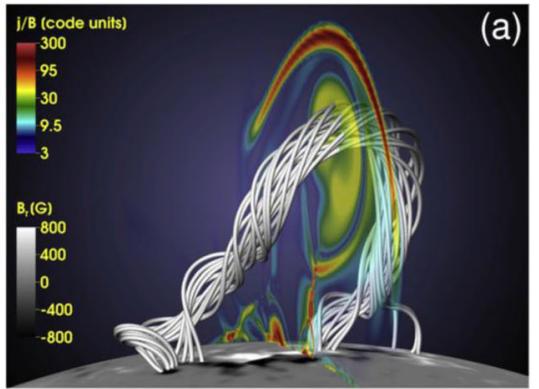

(b)

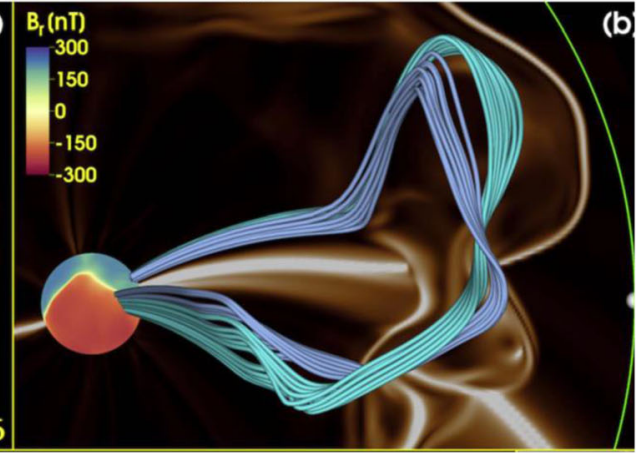

(d)

Fig. 13 a Initial flux-rope field lines with zero- $\beta$ relaxation; $\mathbf{b}$ field lines of the flux-rope core at $t=164.10$; $\mathbf{c}$ Interplanetary magnetic field and ICME flux rope at $t=256$; $\mathbf{d}$ close-up view on (c), showing two flux bundles at the core of the flux rope (From Török et al. 2018) 
conducted a comprehensive study of CME propagation on 7 March 2011 by performing a simulation from the chromosphere to $1 \mathrm{AU}$. Their simulated results could reproduce many of the observed features both near the Sun and in the heliosphere. Figure 14 a depicts the initial GL flux rope configuration for 7 March 2011 CME event with the central plane showing the radial velocity, and Fig. 14 b compares EUV waves in the simulation and in the SDO/AIA observation.

The Versatile Advection Code (VAC) is a general tool for solving MHD and hydrodynamical problems with astrophysical applications. A variety of numerical schemes are available for users to solve hyperbolic differential equations, including, e.g., TVD-Roe, TVDLF, and flux correction of transport (FCT) method. By using the VAC model, Jacobs and Poedts (2012) solved the MHD equations with the inner boundary of the domain locating at the low solar corona. They investigated the effect of new flux emergence on a magnetic system that possessed a 3D topology favorable for the breakout scenario, which was suitable for the 'breakout' CME scenario to work. Keppens et al. (2012) implemented a block-based AMR on the parallel VAC model using the Message Passing Interface library (MPI-AMRVAC), which has been used to provide interplanetary space weather forecasting models with relative accurate time-dependent boundary conditions of erupting magnetic flux ropes in the upper solar corona. Pagano et al. (2015) performed a

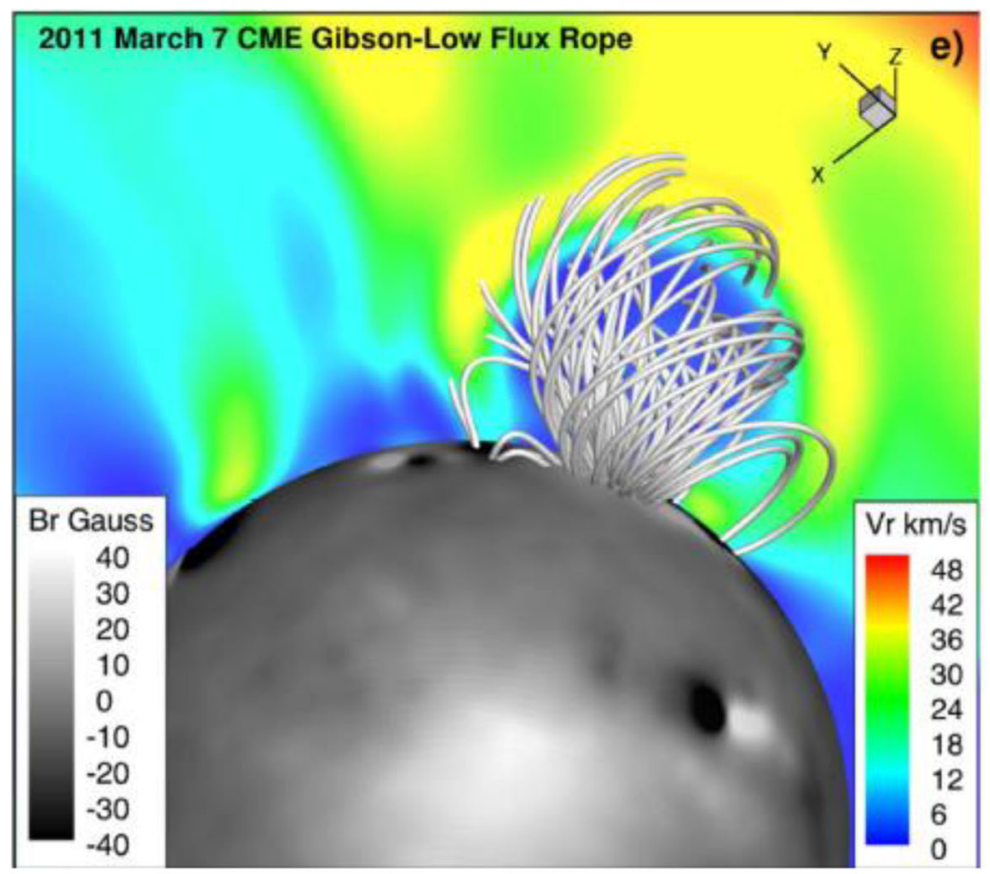

(a)

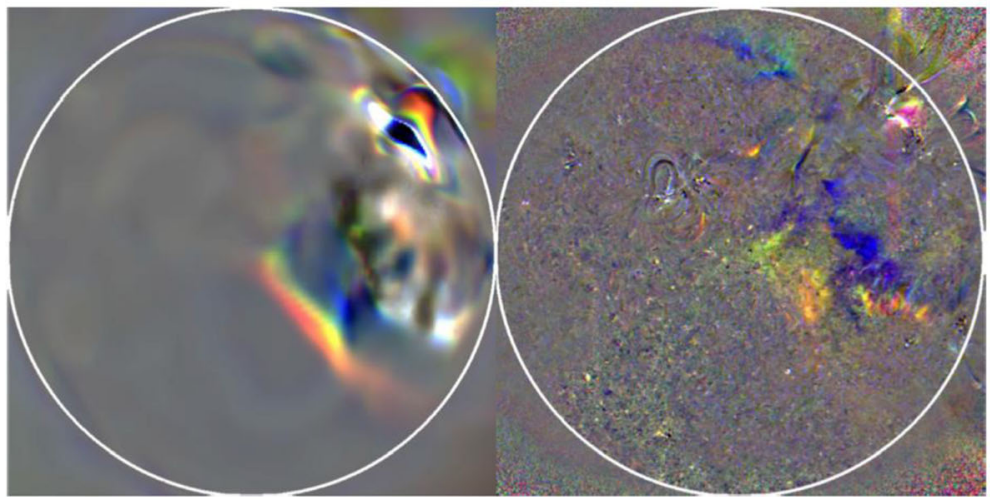

(b)

Fig. 14 a Initial GL flux rope configuration for 7 March 2011 CME; b EUV waves in the simulation (left) and in the SDO/AIA observation (right) (From Jin et al. 2017a) 
3D MHD simulation of a flux rope ejection where a CME was produced by using the MPI-AMRVAC. Their results showed that the polarization ratio technique could reproduce the position of the center of mass along the line of sight with a relative high accuracy and studied the propagation of the CME in the real 3D direction.

Singh et al. $(2019,2020)$ modified the Gibson-Low flux rope by constraining the poloidal and toroidal fluxes of the initial flux rope using eruption data such as total reconnected flux (Gopalswamy et al. 2018c) and flux in the core dimming regions (Webb et al. 2000; Dissauer et al. 2018; Kay and Gopalswamy 2018). The modified spheromak has the option to control the helicity sign of flux ropes, which can be derived from line-of-sight magnetograms. Singh et al. (2020) simulated the 2012 July $12 \mathrm{CME}$ and showed that they can reproduce the properties of the CME in the coronagraph FOV. They created a solar wind background from 1.03 Rs to 30 Rs using solar synoptic magnetograms. Then the flux rope model is inserted into the domain, allowing it to erupt as a CME due to pressure imbalance. They used the Multi-Scale Fluid Kinetic Simulation Suite (MS-FLUKSS, Yalim et al. 2017), which is a highly parallelized code suitable for MHD treatment of plasma and fluid.

Wu et al. (2016c) presented a 3D MHD simulation based on an observed eruptive twisted flux rope deduced from solar vector magnetograms. They combined a datadriven flux rope model for the CME initiation and a global coronal-heliosphere evolution model to track the propagation of the CME. They selected the CME event on 6 September 2011 to test this model, and their simulation results suggested that the flux rope evolution model produced the physical properties of a CME, and the morphology resembled the observations made by STEREO/COR1.

By using the 3D IN-TVD-MHD model (Shen et al. 2018b), Liu et al. (2019a) established a CME flux rope model based on the graduated cylindrical shell (GCS) model and applied it into the numerical simulation on the propagation and deflection of the fast CMEs in the interplanetary space from $0.1 \mathrm{AU}$ to $1 \mathrm{AU}$.

\subsubsection{Spherical plasmoid/magnetized plasma blob} Besides the cone model and the flux rope models, the spherical plasmoid model and the magnetized plasma blob are also popular CME initialization models used in recent years (Shen et al. 2011a; Shen et al. 2011b; Zhou et al. 2012; Shen et al. 2012a; Zhou and Feng 2013; Shen et al. 2014; Zhou et al. 2014; Kataoka et al. 2009; Shiota and Kataoka 2016; Chané et al. 2006). Similar to the flux rope model, they incorporate an internal magnetic field and require the associated parameters. Initial 3D MHD simulations were performed by Groth et al. (2000) and Manchester et al. (2004).
By using the 3D SIP-CESE MHD model with the spherical plasmoid mimicking CME initiation model, the time-dependent propagation of the Sun-Earth connection CME events, such as 4 November 1997, 12 May 1997, and 2010 April 3 CME events were investigated (Zhou et al. 2012; Zhou and Feng 2013; Zhou et al. 2014). And their simulated results provided a relatively satisfactory comparison with the Wind spacecraft observations.

By using the 3D COIN-TVD MHD model with the magnetized plasma blob as CME initialization model, Shen et al. (2011b) and Shen et al. (2014) simulated the time-dependent propagation of single CME events, and the interaction of two CMEs events, such as 4 April 2000 and 12 July 2012 CME events, and 28 March 2001 CME-CME interaction event. Their simulation could reproduce relatively well the real 3D nature of the CME in morphology and their evolution from the Sun to the Earth.

A spheromak-type magnetic flux rope was also taken as the magnetic field structure of the initial CME model by Kataoka et al. (2009) and Shiota and Kataoka (2016), to simulate the propagation of the CME by using the SUSANOO model.

Recently, a spheromak model was included in EUFHORIA (Verbeke et al. 2019a) and shown to work in term of comparison with in situ measurements for specific case studies for Sun-to-Earth propagation of CMEs (Scolini et al. 2019c; Palmerio et al. 2019).

4.2.1.4 Reconstructions of coronal magnetic fields In order to extrapolate the coronal magnetic field from photospheric vector magnetograms based on the nonlinear force-free method, Jiang et al. (2011) and Jiang and Feng (2012) Jiang and Feng (2012) exploited the CESEMHD model to solve the zero-beta MHD equations with a fictitious frictional force and make reconstructions of coronal magnetic field, which was called as CESE-MHD nonlinear force-free field (CESE-MHD-NLFFF) model (also see (Jiang et al. 2013a, ; Jiang et al. 2014; Jiang and Feng 2014). By using the CESE-MHD-NLFFF model combing the vector magnetograms observations, a series of simulations were carried out to investigate, among others, the 3D magnetic field of NOAA AR 11117 on 25 October 2010, formation and eruption of the active region sigmoid in AR 11283, a large-scale pre-flare current sheet in NOAA AR 11967, and the evolving magnetic topology for an X9.3 eruptive flare from geoeffective AR 12673 that occurred on September 6, 2017 (Jiang et al. 2012; Jiang et al. 2013b; Jiang et al. 2016, 2017, 2018). Their simulations could qualitatively reproduce the basic structures of the 3D magnetic field; the current sheet in the corona as well as providing insight into the magnetic mechanism of solar flares; the spatial location, the temporal separation of the observed flare ribbons, as well as 
the dynamic boundary of the flux rope's feet by mapping footpoints of the newly reconnected field lines. Figure 15 presents the comparison of the modeled magnetic field with the observed features of the solar corona prior to the flare field lines (Jiang et al. 2018). Several other studies also reconstructed the 3D coronal magnetic field of AR 12673 and addressed the possible magnetic processes responsible for the X9.3 eruptive flares (Inoue et al. 2018; Liu et al. 2018b; Yan et al. 2018; Mitra et al. 2018; Hou et al. 2018).

By using MPI-AMRVAC MHD code to the reduced MHD equations with only the density, velocity, and the magnetic field, and without the gradient of gas pressure and gravity, and the energy equation, Guo et al. (2019) developed a data-driven MHD model with the zero- $\beta$ approximation. The initial condition is provided by a nonlinear force-free field derived from the magnetofrictional method based on vector magnetic field observation from SDO. Their MHD simulation was carried out for AR 11123 observed on 11 November 2010 and could reproduce the eruption process of the magnetic flux rope.

Other works that have more realistic magnetic field evolution models into the CME model include Price et al. (2019), Pomoell et al. (2019), Hayashi et al. (2018) and Hayashi et al. (2019) among others.
4.2.1.5 Other CME models The HAFv.2+3D MHD model has been used to study a variety of solar eruptive events, such as the interplanetary evolution of the observed geoeffective CME during 1-4 August 2010 (Wu et al. 2011), and the effects of the coronal hole on CME/ shock morphology in the inner heliosphere with 7 March 2011 solar events (Wood et al. 2012). Liou et al. (2014) employed the model to investigate the propagation of the extremely fast backside CME event on 23 July 2012 and the modeled results were in agreement with the in situ measurement from STEREO-A. Specially, Wu et al. (2017) investigated the CME encountered by the Wind spacecraft on 9 September 2011 in detail and verified the association of the short-duration $(\sim 35 \mathrm{~min}) \mathrm{ex}-$ tremely dense pulse (with a peak of $\sim 94 \mathrm{~cm}^{-3}$ ) with the heliospheric plasma sheet compressed by the interplanetary shock.

By injecting a CME from the bottom boundary, Wiengarten et al. (2015) incorporated turbulence transport into the CRONOS model and investigated the effects on the turbulence evolution for transient events from 0.1 AU to $1 \mathrm{AU}$. Their study found that the CME-associated shock increased the turbulence levels and inhibited the cross helicity. They also indicated that researches on the large-scale structures associated with CMEs did not need to consider the turbulence transport effects due to the

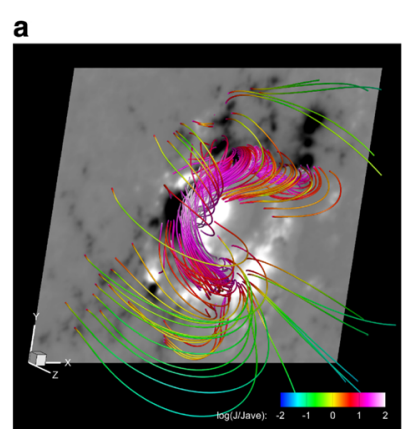

d

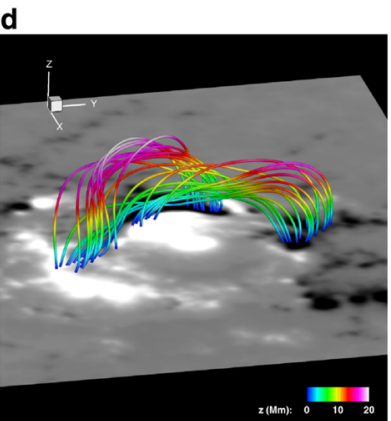

c

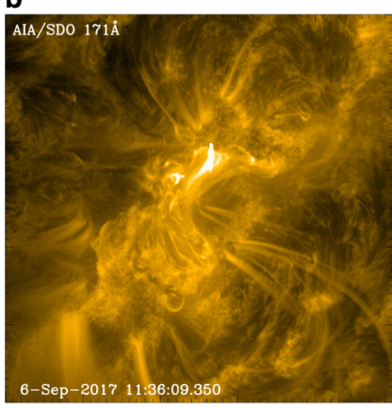

e
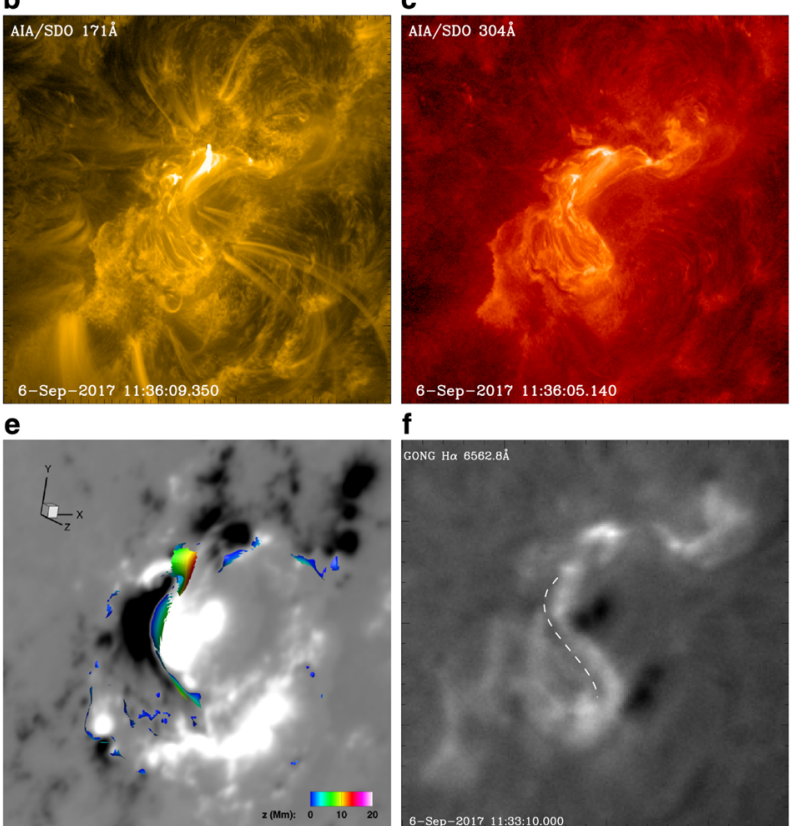

f

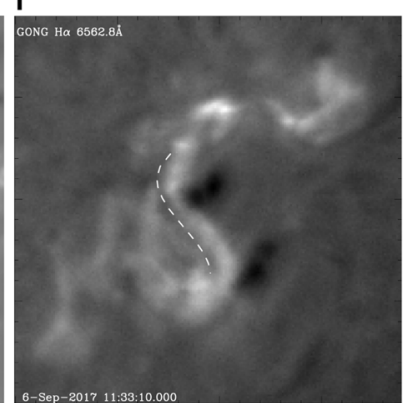

Fig. 15 Comparison of the reconstructed magnetic field with the observed features of the solar corona prior to the flare. a SDO view of sampled magnetic field lines of the CESE-MHD-NLFFF reconstruction. b and c SDO/AIA $171 \AA$ and $304 \AA$ images of the pre-flare corona. d The low-lying magnetic field lines in the core region. The field lines are color-coded by the value of height z. e Locations of dips in the magnetic field lines; the color indicates the value of height z. $\mathbf{f}$ GONG Ha image of the active region. The dashed curve denotes the location of a long filament (From Jiang et al. 2018) 
absence of strong back-reaction of the turbulence on the large-scale structures.

\subsubsection{Modeling the interaction between CMEs and solar wind structure (CIR, HCS)}

Previous numerical studies have shown that both the corotating interaction region (CIR) and heliospheric current sheet (HCS) structures of the background solar wind could play a substantial role in the propagation of CMEs and their geoeffectiveness (Odstrčil et al. 1996; Odstrcil et al. 2004). Therefore, the MHD simulation on the interaction between CMEs and the solar wind structure (e.g., CIR, HCS) is one of the important aspects in the CME simulations and has achieved a lot of progress in recent years.

By using the 3D SIP-CESE MHD model, Zhou and Feng (2017) simulated the propagation characteristics of CMEs launched at different positions in a realistic structured ambient solar wind. By using the HAFv.2+ 3DMHD model, a time series of synoptic photospheric magnetic maps, and the recording of CMEs from STER EO/COR2, Wu et al. (2016c) simulated the Sun-to-Earth propagation of multiple CMEs and their associated shocks in September 2011. Their simulation found that the evolution of the CME-driven shock and its interaction with the HCS and the non-uniform solar wind could explain time-intensity profile of the high-energy $(>10 \mathrm{MeV})$ solar energetic particles (SEPs), and the sector boundary acted as an obstacle to the propagation of SEPs. Further, Wu et al. (2016c) employed the model to study 12 CMEs and their associated shocks in September 2011. The results demonstrated that the background solar wind speed was an important controlling parameter in the propagation of interplanetary shocks and CMEs.

Using 2.5D version of VAC MHD model, Zuccarello et al. (2012) and (Bemporad et al. 2012) numerically studied the role of streamers in the deflection of CMEs or multiple CMEs. Their results showed that the CME deflected toward the current sheet of the larger northern helmet streamer due to an imbalance in the magnetic pressure and tension forces and finally gets into the streamer. As pointed out by (Zuccarello et al. 2012), during solar minima, even CMEs originating from high latitude could be easily deflected toward the HCS, eventually resulting in geoeffective events, and that this latitudinal migration depended on both the strength of the large-scale coronal magnetic field and the magnetic flux of the erupting filament.

Zhuang et al. (2019) simulated the deflection of CMEs with different speeds in the interplanetary space using a 2.5D MHD simulation. Their simulation confirmed the existence of the CME deflection in the interplanetary space, which was related to the difference between the
CME speed and the solar wind speed. They found that a CME, which traveled slower or faster than the solar wind medium, would be deflected to the west or east; and the greater the difference was, the larger the deflection angle would be. Liu et al. (2019b) simulated the propagation and deflection of the fast CMEs interacting with CIR in the interplanetary space by using the 3D IN-TVD-MHD model and the CME flux rope model based on the GCS reconstruction. Their simulation results showed that when the fast CME hit the CIR on its west side, it would deflect eastward, and the deflection angle would increase compared with the situation without CIR.

\subsection{Modeling CME-CME interactions}

Observational and numerical studies have shown that the kinematic characteristics of two or more CMEs may change significantly after the CMEs interaction. The CME-CME interaction is always associated with complex phenomena, including magnetic reconnection, momentum exchange, energy transfer, the propagation of a fast magnetosonic shock through magnetic ejecta, and changes in the CME expansion, and so on (Lugaz et al. 2017; Shen et al. 2017). Numerical modeling, which always yields the observed complexity, has been proven to be a useful tool to understand and determine the dynamical evolutionary processes of the CME-CME interaction.

Webb et al. (2013) tracked the propagation of multiple CMEs of late July to early August 2010 in the inner heliosphere by comparing the results from the ENLIL model, 3D reconstruction techniques based on a kinematic solar wind model, and in situ results from multiple spacecraft. By using WSA-ENLIL+Cone model based on coronagraph image observations, Werner et al. (2019) modeled the multiple CME interaction event on 6-9 September 2017. The predicted arrival time of the first interplanetary shock was drastically improved, while the background solar wind preconditioned by the passage of the first interplanetary shock likely caused the last CME to experience insignificant deceleration and led to the early arrival of the second interplanetary shock.

Using the COIN-TVD MHD model, Shen et al. (2011c) and Shen et al. (2012) simulated the interaction of two CMEs in interplanetary space, analyzed variations of different forces during the interaction, and found that the momentum exchange during the collision of two CMEs was very important for the deceleration and acceleration of the CMEs. The 3D COIN-TVD model was also used to study the superelastic collisions of CMEs in the heliosphere (Shen et al. 2013a). Results showed that the collision led to extra kinetic energy gain by $3-4 \%$ of the initial kinetic energy of the two CMEs, which suggested that 
the collision of CMEs could be superelastic. Shen et al. (2016) furthered the dependence of CMEs' collision type on the ratio of the CME's kinetic energy to the CME's total energy.

By employing the SUSANDOO model, Shiota and Kataoka (2016) reproduced the propagation and interaction process of multiple CMEs associated with the highly complex active region NOAA AR 10486 from $30 \mathrm{Rs}$ to $430 \mathrm{Rs}$ in October to November 2003. Their simulation results could successfully provide reasonably good results for velocity and the profile of southward magnetic field component of the Halloween Event on 29 October 2003. The simulation also indicated that the propagation of the following CME was significantly affected by the trails of the preceding CMEs. Lugaz et al. (2013) used the SWMF to study the influence of the relative orientation of the two interacting CMEs on their interaction and the resulting structure. In addition to the well-studied multiple-MC event, they described other potential structures, further compared with actual CME measurements in Lugaz and Farrugia (2014).

The September 2017 series of events, which resulted in one of the largest geomagnetic storms of solar cycle 24 , has been investigated by means of numerical simulations, focusing on the CME-CME interaction by a number of groups. Scolini et al. (2019a, b, c) used EUHFORIA to study how complex interactions between multiple interacting CMEs on their way to Earth may result in an intensification of the geo-effectiveness potential of such multiple-CME events. Werner et al. (2019) used the ENLIL model to investigate how the succession of CMEs in early September 2017 resulted in one of the largest geo-effective periods of solar cycle 24 . They specifically focused on the importance of pre-conditioning of previous, non-interacting CMEs on the propagation of the following fast CMEs, confirming past work, both based on simulations (Lugaz et al. 2005; Tóth et al. 2007) and measurements Liu et al. (2019a).

\subsection{Conclusions and future prospects}

In the past five years, the main developments in the investigation of CME propagation and the background solar wind by means of numerical simulation have been as follows.

There has been a significant increase in the number of 3D MHD codes that have been successfully used to simulate the Sun-to-Earth propagation of CMEs as well as the background solar wind; this has been the case most notably in Europe with EUHFORIA, Japan with SUSANOO, and China with IN-TVD MHD. In addition, a number of existing MHD codes have been adapted to investigate the heliospheric propagation of CMEs, including LFM into LFM-Helio and MAS into MAS-Helio.
Heliospheric codes (starting typically at $0.1 \mathrm{AU}$ ) have been used with spheromak and/or flux rope CMEs, which bridges the gap between computationally intensive Sun-to-Earth simulations and heliospheric simulations with cone models. These types of simulations may be used to investigate the magnetic field configuration inside CMEs as well as their arrival time and are more physically consistent when investigating CME-CME interaction than simulations where the CMEs do not have internal magnetic fields.

In parallel, there has been an effort to make the CME initialization quicker and easier to perform in coronal codes using out-of-equilibrium flux ropes, especially with EEGL in the SWMF and within the MAS code. This paves the way for future, real-time Sun-to-Earth simulations with magnetized CMEs initiated based on magnetograms, EUV images, and early coronagraphic images. It is well known that major changes in the CME properties, including its speed and orientation, may occur below $0.1 \mathrm{AU}$ where heliospheric models are initiated. At this time, it is however unclear whether simulations with magnetized CMEs initiated at $0.1 \mathrm{AU}$ using multi-viewpoints coronagraphic measurements (as described in point 2) will perform worse than simulations with magnetized CMEs initiated at the solar surface in term of space weather forecasting capabilities. The number of Sun-to-Earth simulations of CMEs initiated at the solar surface with a realistic model is still relatively low, even though there has been effort in presenting the results near $1 \mathrm{AU}$ of more complex initiation mechanisms, as done for example in Török et al. (2018).

The solar wind background plays an important role in constraining CME propagation, in particular, in predicting the arrival times of CMEs. There has been significant new physics included in the solar wind models, including more advanced thermodynamics treatment, the inclusion of Alfvén waves and the new treatment of the inner boundary. However, there has not been significant work quantifying how these new additions affect the CME propagation and the resulting structure near $1 \mathrm{AU}$.

Lastly, there has been progress towards coupling timedependent magnetic field models with coronal models and heliospheric models. This is already the case for the background coronal and interplanetary magnetic field with ADAPT coupled to a number of MHD models, which has been shown to result in more accurately modeled heliospheric current sheets. Initiating CMEs by means of magnetofrictional or other self-consistent models based on solar observations or flux emergence may lead the way for a better physical understanding of CMEs and is probably the only way space weather forecasting could provide information before the launch of a CME. We expect further improvements towards this coupling in the next few years. 


\section{Campaign study of Sun-Earth connection events 5.1 Introduction}

The task of ISEST Working Group 4 (Campaign Events) was to integrate theory, simulations, and observations to better understand the chain of cause-effect activity from the Sun to Earth for carefully selected events. ISEST provided "textbook," or well-understood, Sun to Earth cases to the community, but WG 4 also examined more controversial events, such as stealth CMEs and problem ICMEs, to enhance our understanding. This includes analyzing the difficulties in linking CMEs to ICMEs, which are usually observed only in situ.

WG 4 classified the studied events into three general categories: (1) Possible "textbook" cases in which the complete chain of a well-observed event is relatively well understood from its solar source, through its heliospheric propagation, to its geo-effects. These cases involve forecasts that are successful in a general way. (2) Cases in which there were problems understanding the complete chain, but which we think we now understand. Thus, something was missing in the chain of a wellobserved event but, in retrospect after analysis, we now understand why. These cases usually involve forecasts that failed because they were not geoeffective, or were otherwise not accurate. (3) Finally, there are problem cases in which the chain is not complete and we still do not understand why. In the next two sub-sections, we briefly summarize the results for each type of the events, which are discussed in detail in Webb and Nitta (2017) (hereafter WN17) and Nitta and Mulligan (2017) (hereafter NM17). WN17 studied six cases during the rise of Solar Cycle 24 that highlight forecasting problems. The six events were chosen to illustrate some key problems in understanding the chain from solar cause to geoeffect. NM17 studied stealth, or problem CMEs that have no clear Low Coronal Signatures (LCS).

Table 5 is a summary of the 14 campaign events that were discussed and analyzed by WG 4 . These studies have resulted in many presentations and papers in the literature. The first column group gives the Event Number and the range of dates from the solar source to any geo-effect. The second column group summarizes the source activity, the third the geo-response, followed by the storm peak Dst, if any, and the peak Kp and G indices (see below). Finally, at the right is given our estimate of the degree of forecast success. The first six events were chosen as VarSITI-wide Campaign Study Events because they had certain space weather effects of interest to one or more of the other three VarSITI projects. A focus of the WG 4 studies was to understand the Sunto-Earth cause-effect chain for five of these 6 campaign events. The other 8 of the 14 events were chosen because of particular aspects of interest to ISEST WG 4 that help elucidate the Sun-to-Earth chain.
Only the first 11 events were included at the time of writing of the WN17 paper. The 5 events studied by WN17 are highlighted in red. Three of the events, as well as others, were described by NM17 and are marked in purple. The October 2012 problem event was discussed in both papers.

This Section is organized as follows. The next subsection provides a summary of the Campaign events WG 4 studied during the rise of Solar Cycle 24 that highlight forecasting problems. Subsection 5.3 summarizes the results from the Nitta and Mulligan study of stealth CMEs. The results are discussed in subsection 5.4.

\subsection{Understanding problem forecasts}

WN17's six events were selected to illustrate the range of problems that can occur in understanding the complete chain of activity from its source region(s) at the Sun, its propagation through the heliosphere, to its effects at Earth. Likely source CMEs were identified in all six cases, but related solar surface activity ranged from uncertain or weak to X-class flares. The geoeffects ranged from no effects to severe effects, such as the two Sun-Earth events in 2015 that caused "superstorms." For each event, they noted the official NOAA forecast that was issued after the solar source eruption but before its arrival at Earth, and whether the forecast was successful or was problematic in some important manner. Summaries of these forecasts are available under Reports of Solar and Geophysical Activity (RSGA) through the Space Weather Prediction Center (SWPC) site: ftp://ftp. swpc.noaa.gov/pub/warehouse/.

Event \#1, 12-14 July 2012, was considered a classic textbook event, in that we observed the complete chain of a well-observed Sun-to-Earth event, from its solar source, through heliospheric propagation, to its geoeffects (Figs. 1 and 16). The propagation kinematics, flux rope eruption, and MHD modeling for this event were well studied by WG 4 members (Gopalswamy et al. 2013a; Hess and Zhang 2014; Shen et al. 2014; Möstl et al. 2014; Cheng et al. 2014; Hu et al. 2016; Marubashi et al. 2017) and others. On 12 July 2012, an eruptive X1.4 flare occurred in AR 11520 (S17 W08) with an X-ray peak 16:45 UT. Later during its rotation, this same active region produced several strong flares and CMEs. One was the 23-24 July CME (\#8), aimed at the STER EO-A and one of the fastest, most energetic CME ever observed (e.g., Baker et al. 2013; Liu et al. 2014). On 14 July the CME arrived at L1 with a shock observed by the Wind spacecraft at 17:38 UT, followed by the shock sheath and a 2-day long magnetic cloud. This ICME drove a moderate, long-lived geomagnetic storm with peak Dst $=-127 \mathrm{nT}$ on 15 July and with a duration of several days. The NOAA forecast was mostly successful 
Table 5 ISEST/MiniMax WG 4 Campaign Events

\section{ISEST / MiniMax WG 4 Event List}

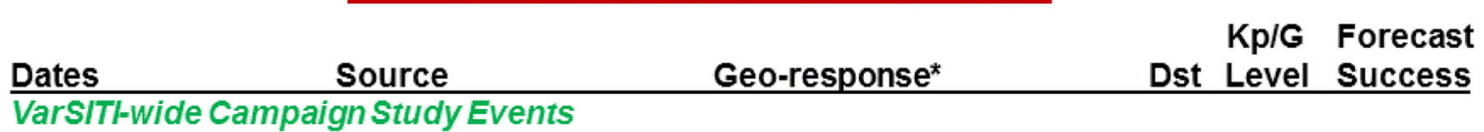
1) 2012 July $12-14$
2) 2012 Oct. $4-8$
3) 2013 March $15-17$
4) 2013 June 1
5) 2015 March $15-17$
6) 2015 June $22-24$

$\mathrm{X} 1$ flare, wave, fast CME

Shock, MC, Strong storm

$-127 \quad 7 / G 3$

Under-predicted

CME; weak surface signs.

Shock, MC, HSS, Moderate stm

$-105 \quad 6+/ G 2$

Slow CME on 27 May?

CHinfluence?

Shock, MC? SEP, Strong storm

$-1326+/ G 2$

Under-predicted

C9;C2 fl, wave, EF, fast CME Shock, sheath, MC, Severe storm -222 8+/G4

2 M-fls, waves, fast halo CMEs Shock, sheath, MC, SEP,

\section{Failed-not pred.}

Under-predicted

\section{OtherISEST/MiniMax Study Events}

7) 2012 March $7-9$
8) 2012 July $23-24$
9) 2014 January 6
10) 2014 January $7-9$

11) 2014 Sept. $10-13$

12) 2015 January $3-7$

13) 2016 October $8-12$

14) 2017 Sept $4-10$

\begin{abstract}
X5 flare, wave, fast CME 2 flares? Wave, EFs CME $<2000 \mathrm{~km} / \mathrm{s}$, over WL $\mathrm{X} 1 \mathrm{fl}$, wave, fast asym halo
\end{abstract}

X2 flare, wave, sym halo Slow CME

Slow CME

Act. series; 3 S-E evts.
Severe storm

$-2048+/ G 4$

\begin{tabular}{|c|c|c|c|}
\hline Shock, MC, Strong storm & -131 & 8/G4 & \\
\hline Extreme ST-A event; "Stron & $\mathrm{rm}^{\prime \prime}(\mathrm{C}$ & Carr.-ty & $\cdots$ \\
\hline GLE at Earth & No & --- & \\
\hline Shock, SEP. No storm- $\mathrm{CH}$ deflecti & tion; & & \\
\hline AR channeling? & No & $\leq 3$ & \\
\hline Shock, MC, Moderate storm & -88 & $7 / G 3$ & Over-predicted \\
\hline Brief ICME, MC, HSS, Mod. stm & -99 & $6+/ G 2$ & \\
\hline Shock, MC, HSS, Moderate stm & -104 & $6+/ G 2$ & \\
\hline hocks, MCs, Strong storm(s), FD & -124 & $8 / G 4$ & \\
\hline
\end{tabular}

Shock, MC, Strong storm $\quad-131 \quad 8 / \mathrm{G} 4$

"

Shock, SEP. No storm- $\mathrm{CH}$ deflection AR channeling?

Mostly successful

$\mathrm{CME}=$ coronal mass ejection; $\mathrm{AR}=$ active region; $\mathrm{EF}=$ erupting filament; $\mathrm{CH}=$ coronal hole; $\mathrm{MC}=$ magnetic cloud; $\mathrm{SEP}=$ solar energetic particle event; $\mathrm{CIR}=$ corotating interaction region; GLE = ground-level event; HSS = high speed stream

xx) Events featured in Webb \& Nitta (2017)

xx) Problem events featured in Nitta \& Mulligan (2017)

for this event, but the storm was only moderate level so slightly under-predicted.

The 21-24 June 2015 case, \#6, was also possibly a textbook event, but it was a compound in situ event at 1 AU resulting from a series of four shocks arriving over a 3-day span, and one likely ICME on 23-24 June. The third shock and ICME were likely produced by a symmetric halo CME on 21 June. Southward field in multiple shock sheaths and the ICME drove a powerful multi-step geomagnetic storm reaching $\mathrm{Kp}=8+$, G4 and

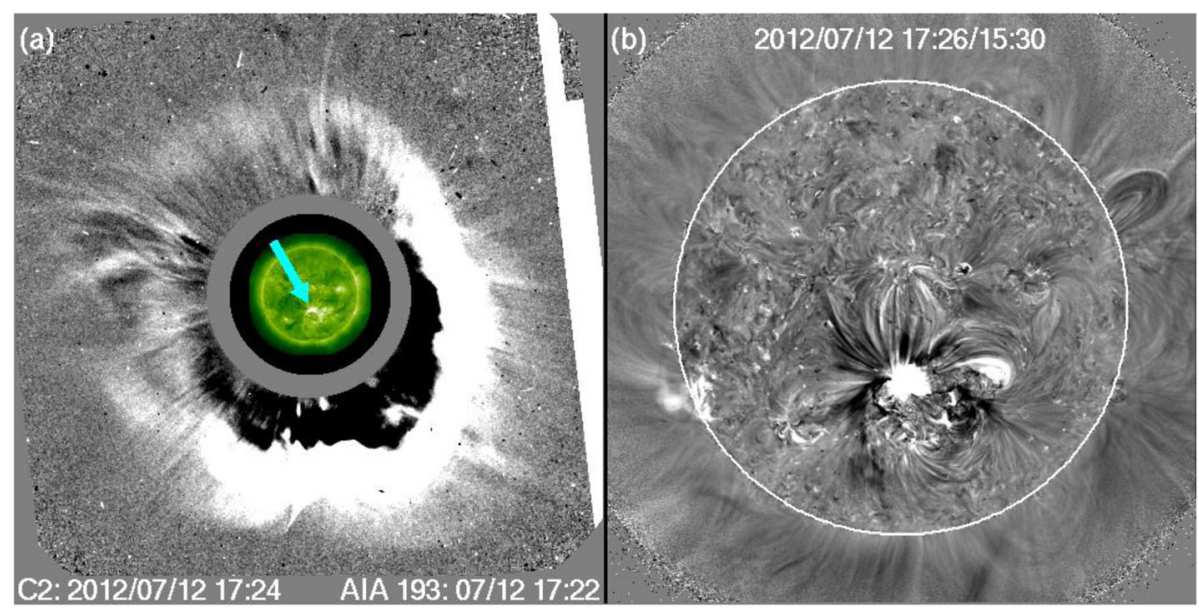

Fig. 16 Images showing the solar source region of Event \#1 on 12 July 2012. Left: SOHO/LASCO running difference image showing the full halo CME on 12 July, 17:24 UT. Superimposed within the occulting disk area is a near-simultaneous SDO/AIA $193 \AA$ image with an arrow pointing to the near-disk center flare and dimming in AR 11520. This image is from the SOHO/LASCO CDAW CME catalog. Right: Enlarged AIA base difference image of the source region 
Dst $=-204 \mathrm{nT}$ on 22-23 June. The NOAA forecast of a severe storm was accurate, but that level was reached a day later than predicted. This severe level was reached because there were multiple shocks and sheaths, strong southward MC fields, and high speed solar wind that acted to compress and enhance the wind structures. Publications of this event include the following (Ying D. Liu et al. 2015; Manoharan et al. 2016; Lugaz et al. 2016; Marubashi et al. 2017; Gopalswamy et al. 2018c).

The 15-18 March 2015 case, \#5, was initially a problem event, but we now understand why. A slow $(350 \mathrm{~km}$ $\mathrm{s}^{-1}$ ) CME occurred to the south-southwest late on 14 March likely associated with a small C2.6 flare from AR 12297 at $\mathrm{S} 21^{\circ} \mathrm{W} 20^{\circ}$ with a small filament eruption. Then on 15 March, $\sim$ 01:36 UT, a fast $\left(1120 \mathrm{~km} \mathrm{~s}^{-1}\right)$, asymmetric halo CME associated with a C9.1 flare erupted from the same active region but was brightest over the west limb. In situ L1 observations showed a strong shock at Wind on 17 March at 04:01 UT, followed by an extended sheath then an ICME and a MC later on 17 March. Behind the cloud were a corotating interaction region (CIR) and its high-speed stream (HSS) that likely enhanced the solar wind parameters. This most severe storm of Solar Cycle 24 was very much under-predicted, in terms of both its magnitude and early time of arrival. Thus, this was a problem forecast. The CMEs may or may not have interacted near the Sun. There were two detailed papers by Liu et al. (2015) and Wang et al. (2004) arguing either side of that dispute. It is likely that during transport to Earth there was interaction with a CIR and deflection toward Earth. Other papers analyzing the $\mathrm{MC} /$ flux rope at $1 \mathrm{AU}$ include (Marubashi et al. 2016; Marubashi et al. 2017; Wu et al. 2016a).

Although not one of WN17's primary study events, Event \#10, 7-9 January 2014, was at the time considered a problem event, but is now understood. It was a problem because a large storm was predicted but none occurred! Unlike the March 2015 case in which the CME was deflected toward Earth, in this case, the source flare and EUV wave were Sun-centered but the CME was offset to southwest, possibly deflected by a $\mathrm{CH}$ and/or channeled by strong AR magnetic fields, and thus missed the Earth (Gopalswamy et al. 2014a, b; Möstl et al. 2015; Wang et al. 2015; Mays et al. 2015a).

The 10-12 September 2014 case, \#11, was initially a problem event, but we now understand why. On 10 September 2014, an X1.6 flare erupted in AR 12158, associated with a fast $\left(1400 \mathrm{~km} \mathrm{~s}^{-1}\right)$ symmetric halo CME. The event was centered on the disk and had a large dimming region and a rapidly expanding coronal wave. This event seemed like a textbook example, with a major storm predicted, followed by a strong shock and longduration MC hitting Earth on 12-13 September.
However, the storm was minor because the sheath and $\mathrm{MC}$ magnetic fields were northward $\left(+B_{Z}\right)$, so the storm was over-predicted.

WG 4 members tried to use polarity inversion line data to predict the expected flux rope orientation at 1 $\mathrm{AU}$, but no consensus was reached. It was found that the flux rope fit better to a later, smaller flare in the same AR (Marubashi et al. 2017; Cho et al. 2017). Thus, the effects at L1/Earth were likely due to the interaction of the large and small eruptions; see Webb and Nitta (2017) for details. An et al. (2019) used observations of the large event in a 3D MHD simulation to compare with a set of the ICME parameters at 1 AU. However, they did not consider any interaction of the solar events or forecasting problems per se.

The 4-9 October 2012 case, \#2, was initially a problem event but we now understand why. The source CME and resulting ICME that drove a small, two-step geostorm $(\mathrm{Kp}=6+, \mathrm{G} 2)$ were identified, but the storm was slightly under-predicted. There were weak and multiple surface signatures and the CME was initially very slow, leading to uncertainties in the arrival time. Marubashi et al. (2017) studied the ICME for this event and fit a portion of it as a flux rope.

Finally, the 27 May-1 June 2013 case \#4, was a problem event, which we still do not fully understand. During this entire period, NOAA/SWPC did not forecast any important geoactivity. But on 1 June, there was a brief but strong storm (G3) that reached $\mathrm{Kp}=7$ and Dst $=-119 \mathrm{nT}$. A possible source was a slow CME on 27 May, but the associated surface features were unclear. There was also likely influence from a large coronal hole that led to interaction with a CIR or HSS at Earth (Gopalswamy et al. 2015a, b, c), with a likely embedded ICME and flux rope (Marubashi et al. 2017; Nitta and Mulligan 2017). The ultimate cause of the strong storm remains unclear.

Events \#12 and 13 in the WG 4 table are discussed as stealth CMEs in the next section. The last "event," \#14, was actually a series of major flare ( $\mathrm{M}$ and $\mathrm{X}$-class)CME events from 4-10 September 2017 resulting in shocks, MCs, and a Forbush decrease at 1 AU. X flares occurred on 6, 7, and 10 September. One notable feature of this series of events was the timing of the $1 \mathrm{AU}$ arrival of the shock from the second CME (associated with a X9.3 flare) that was nearly simultaneous with when the magnetic field of the ICME from the earlier CME (associated with a M5.5 flare) turned southward. This apparently enhanced the net geoeffectiveness of these individual events, but it is presently very challenging to forecast the timings of the successive phenomena, let alone individually. This event period occurred too late in the ISEST interval to be extensively studied by WG 4, but WG 4 members contributed to several papers 
mostly analyzing the $\mathrm{X}$ flares and CMEs at the Sun. Recently, some WG4 members (Schmieder et al. 2020) studied the geoeffectiveness of a set of X-class flares in 2002. Most had near-limb solar sources but half also had fast, halo CMEs. They conclude that these were "problem" events in that the usual solar proxies were not sufficient to forecast the rather weak geoeffects.

\subsection{Study of stealth CMEs; those without clear low coronal signatures}

A related study of the Campaign group was of the origin of CMEs that were not accompanied by obvious low coronal signatures (LCSs), but produced appreciable geoeffects at $1 \mathrm{AU}$. These CMEs characteristically start slowly. In several examples, extreme ultraviolet (EUV) images taken by the Solar Dynamics Observatory (SDO)/Atmospheric Imaging Assembly (AIA) revealed coronal dimmings and post-eruption arcades using difference images with sufficiently long temporal separations, which are commensurate with the slow initial development of the CME. Images from SECCHI EUVI and COR coronagraphs provided limb views of Earthbound CMEs. Combined with $\mathrm{SOHO}$ observations, these helped limit the time interval in which the CME forms and undergoes initial acceleration. For other CMEs, we found similar dimming, although with lower confidence of its link to the CME. We note that even these unclear events can result in unambiguous magnetic cloud/flux rope signatures in in situ data at 1 AU. In addition, there was a tendency for the CME source regions to be located near coronal holes. i.e., open field regions. This may have implications for both the initiation of a stealth CME in the corona and its outcome in the heliosphere.

The fact that some events without obvious LCSs produce appreciable geostorms was one of the motivations of the work by NM17, who not only discussed Event \#2 in detail but also described Events \#12 and \#13 and also included Event \#4 in their event list. It is notable that four of the 14 events dealt with by WG 4 were in the category of geomagnetic storms without obvious LCSs. Stealth CMEs are of great scientific interest because they may represent a different class of eruptions than normal CMEs, whose LCSs are unambiguous. Howard and Harrison (2013), however, cautioned that stealth CMEs may be due largely to observational effects (such as limited sensitivity and temperature coverage). Indeed it has been shown that initially unseen LCSs may be revealed after image enhancement or processing (Alzate and Morgan 2017; Nitta and Mulligan 2017). Therefore, it may be more appropriate to use the adjective "stealthy" when the LCSs of the CME are not clearly identified. Regardless of whether they are fundamentally different from normal CMEs or simply represent the low-energy end of a continuous spectrum of events triggered in similar ways (Lynch et al. 2016), stealthy events pose challenges to space weather prediction.

In Event \#2, NM17 found a post-eruption arcade (PEA) sandwiched by coronal dimming regions in AIA images around the time of the first appearance of the CME in LASCO C2 images, using difference image cubes with long ( several hours) temporal separations (Fig. 17). It was found that the PEA and dimming regions delineated a polarity inversion line that looked like a filament channel without a filament. Apart from the base difference images that needed compensation of solar rotation as a result of the long temporal separations, another important point was to use COR-1 data that allowed the determination of the CME lift-off time by observing the eruption from the side. Using AIA base difference images and COR-1 data for several stealth events, NM17 identified similar patterns of a PEA with dimming regions on either side. Figure 18 shows the ICME with MC and fitted flux rope at $1 \mathrm{AU}$.

However, Events \#12 and \#13 were more problematic. In Event \#12, a partial halo CME was linked to the ICME responsible for the strong (Dst $=99 \mathrm{nT}$ ) geostorm. This CME was both very slow and diffuse. Even though one dimming region was clear not only in base difference images but also in intensity images in AIA's $193 \AA$ and $211 \AA$ channels as an augmentation of the south polar coronal hole, its mapping to the CME was not straightforward. Assuming that the dimming regions represent the legs of the erupting flux rope responsible for the CME, we would expect two dimming regions in opposite magnetic polarities. However, no second dimming region was found. Moreover, multiple regions became brighter in base difference images, and it is not possible to determine which ones may represent PEAs. This event also lacked STEREO observations, making it difficult to know the time of the CME liftoff.

Event \#13 involved a full halo CME, which was again slow and diffuse. Without STEREO observations, this could have easily been taken as a backside event because there were no clear changes in the low corona around the time of the CME. NM17 showed two marginal dimming regions in AIA difference images taken $14 \mathrm{~h}$ apart, but did not attach high confidence to them. Event \#4 is controversial as to whether the strong (Dst $=119 \mathrm{nT}$ ) geomagnetic storm was purely CIR-related or enhanced by a small ICME embedded in the solar wind. The most likely CME in the time range in question was observed to head northward, giving an impression that it was not Earth-directed, but it could have deflected equatorward as indicated in STEREO HI data. Marubashi et al. (2017) showed a flux rope fitting, possibly supporting the latter possibility. NM17 located a 


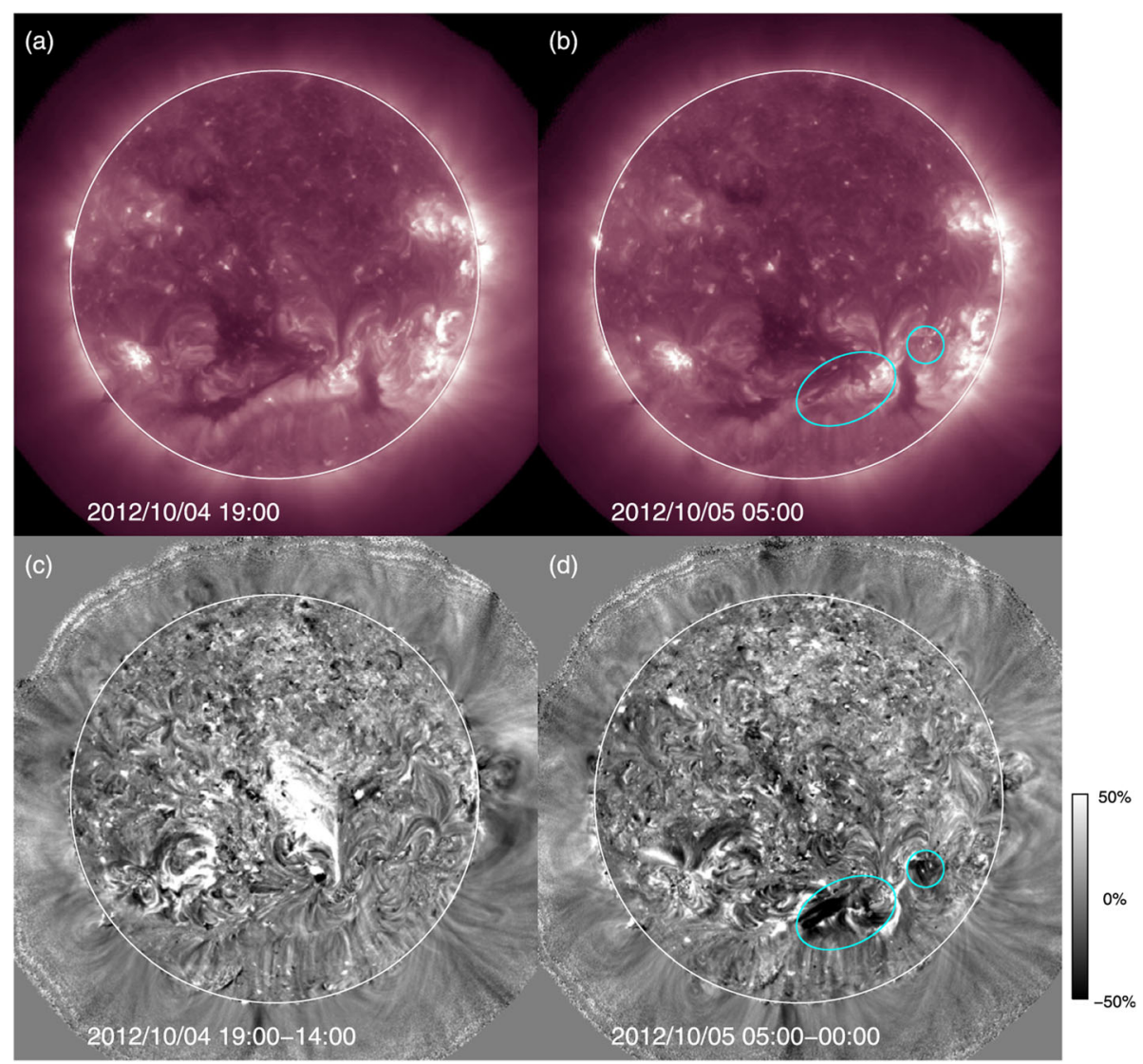

Fig. 17 AIA $211 \AA$ intensity and percent difference images in the upper and lower panels, respectively, taken before and during the CME on 5 October 2012. Differences are made relative to an image $5 \mathrm{~h}$ earlier. The dimming regions found in difference images are encircled in cyan. From Nitta and Mulligan (2017)

dimming region next to the coronal hole as was the case for Event \#12.

\subsection{Discussion}

The goal of ISEST WG 4 was to integrate observations, theory, and simulations to understand the chain of causeeffect dynamics from the Sun to Earth for a few carefully selected (Campaign) events. This should help us develop and/or improve the prediction capability for the arrival of these transient and their potential impacts at Earth.

WG 4 also examined controversial events, such as stealth or "silent" CMEs and problem ICMEs to enhance our understanding. One focus of WG 4 was on why do forecasts fail and how can we improve our predictions. This included analyzing the complications in linking CMEs to ICMEs, usually observed only in situ at 1 AU. Our July 2012 (\#1) and June 2015 (\#6) cases were considered "textbook," but the forecasts were not fully accurate. The June 2015 case involved a compound event that likely enhanced the level to a severe storm. The next three cases, March 2015, \#5, September 2014, $\# 1$, and October 2012, \#2, were all considered problem events that we now understand. In March 2015 two CMEs possibly interacted near the Sun and were deflected by a CIR. In September 2014 the storm was much over-predicted because the shock sheath and MC fields were almost entirely northward $\left(+B_{Z}\right)$. For October 2012 a CME was identified but the surface signatures were multiple and weak leading to uncertainties in the arrival time. Finally, the last case in May-June 2013 (\#4) was a problem event that we still do not fully understand. No storm was forecast but a brief, strong storm occurred. The surface activities associated with a slow CME were unclear as was the cause of the southward field $\left(-B_{Z}\right)$ at 1 AU.

As in several of our cases, we note that about $20 \%$ of important geomagnetic storms have identified ICMEs but no compelling solar signatures. Likewise, Earthaffecting CMEs are sometimes "stealthily" launched without clear LCSs. In our stealth CME study, we demonstrated the need to compare AIA images with long temporal separations to find weak LCSs, especially coronal dimmings and PEAs, in stealthy eruptions or slow CMEs. In addition, STEREO COR data provided the 


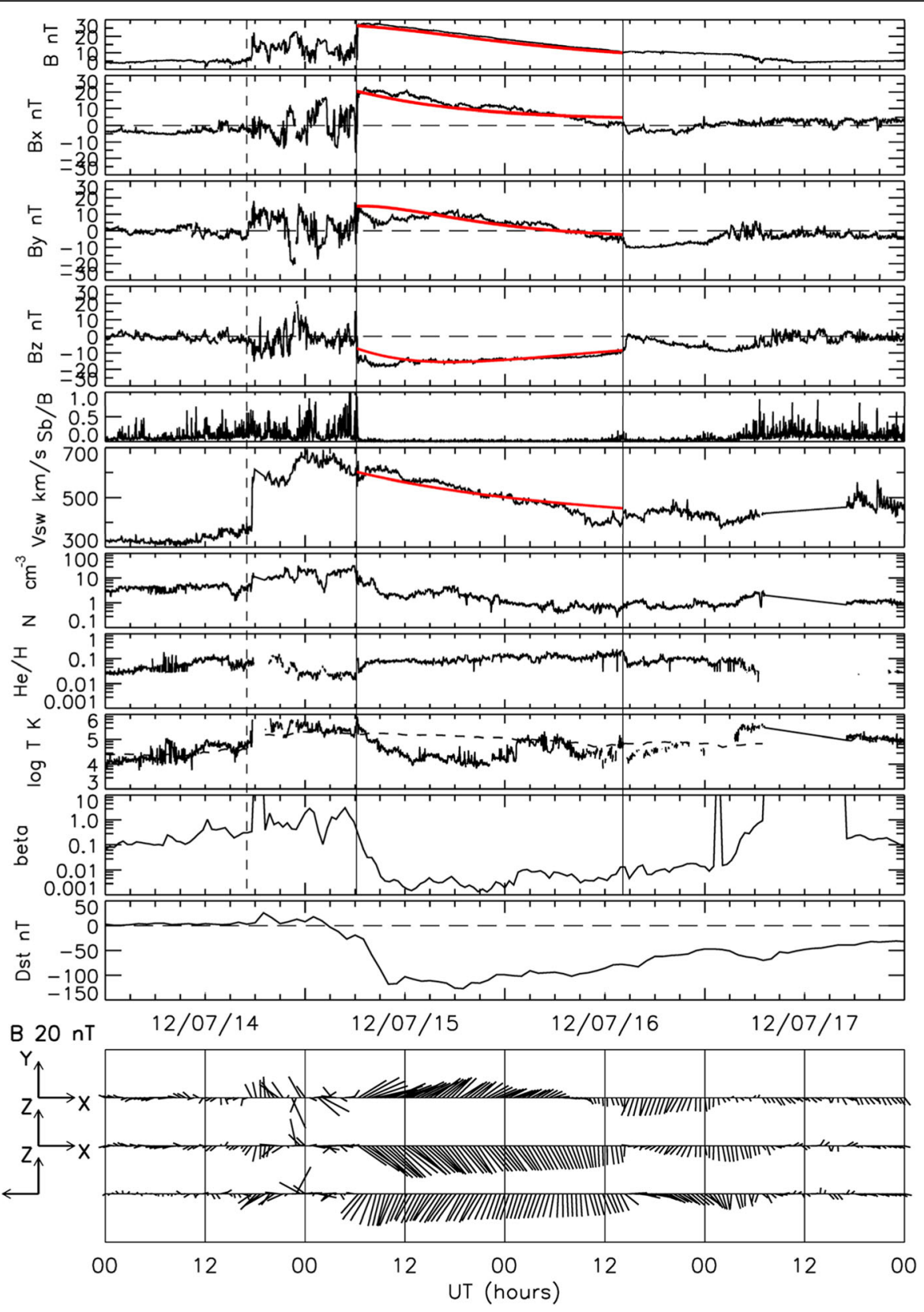

Fig. 18 Solar wind data from Wind and the Dst variation for three days, 8-10 October 2012. From top to bottom are plotted the IMF intensity (B), $X-, Y-, Z$-components in GSE coordinates $\left(B_{x,}, B_{y}, B_{z}\right)$, the degree of field fluctuations defined by the standard deviation divided by averaged intensity obtained from higher time resolution data $(\mathrm{Sb} / B)$, solar wind speed $\left(V_{\text {sw }}\right)$, proton number density $(N)$, number density ratio of He++ to $\mathrm{H}+(\mathrm{He} / \mathrm{H})$, proton temperature, plasma $\beta$, and the Dst index. The vertical dashed line indicates the shock arrival time (on 8 October at 04:12 UT) and the two vertical lines indicate the flux rope (from 8 October at 17:20 UT to 9 October at 18:30 UT). The red curves show the model values obtained from the fitting with a toroidal flux rope model. Adapted from Marubashi et al. (2017)

time range to examine AIA data that matches CME formation and acceleration. We found a tendency for the CME source regions to be located near coronal holes, or open field regions.

Finally, about $10 \%$ of intense storms are due to the compression of fields and plasma by CIRs and their HSSs (Zhang et al. 2007). CIRs played a role in two of our cases. The shock sheath region can also be very important for driving storms as was the case in at least two of our events. A problem is that the sheath fields consist of swept-up coronal and heliospheric material which are hard to predict in advance. Thus, studies of sheath regions are an important, but not poorly understood aspect of space weather forecasting. 


\section{Solar energetic particle events 6.1 Introduction}

Solar Energetic Particles (SEPs) from suprathermal (few $\mathrm{keV}$ ) up to relativistic (few $\mathrm{GeV}$ ) energies constitute an important contributor to the characterization of the space environment. They are emitted from the Sun in association with solar flares and Coronal Mass Ejection (CME)-driven shock waves. SEP radiation storms may have durations from a period of hours to days or even weeks and have a large range of energy spectrum profiles. These events pose a threat to modern technology strongly relying on spacecraft and are a serious radiation hazard to humans in space, and additionally of concern for avionics and commercial aviation in extreme circumstances (Malandraki and Crosby 2018a, b). This section is divided into subsections, devoted to the progress of SEP research from both the observational as well as the theoretical and modeling perspective.

\subsection{State of SEP observations and theory until 2014}

By the end of the 1990s, a two-class paradigm (see Fig. 19) for SEP events was generally accepted (Reames 1999, 2013; Desai and Giacalone 2016). In this paradigm, the gradual events occurred as a result of diffusive acceleration at CME-driven coronal and interplanetary (IP) shocks, while the impulsive events were attributed to acceleration during magnetic reconnection in solar flares. The gradual or CME-related events typically lasted several days and had larger fluences, while the impulsive or flare-related events lasted a few hours and had smaller fluences. Impulsive events were typically observed when the observer was magnetically connected to the flare site, while ions accelerated at the expanding large-scale CME-driven shocks can populate magnetic field lines over a significantly broad range of longitudes (Cane et al. 1988). The distinction between impulsive and gradual SEP events was further justified on the basis of the energetic particle composition and radio observations (Cane et al. 1986). For instance, the flare-related impulsive SEP events were electron-rich and associated with type III radio bursts. These events also had ${ }^{3} \mathrm{He} /{ }^{4} \mathrm{He}$ ratios enhanced between factors of $\sim 10^{3}-10^{4}$, Fe/O ratios enhanced by up to a factor of 10 over the corresponding $\mathrm{SW}$ values and had Fe with ionization states up to $\sim 20$. In contrast, the gradual events were proton-rich, had average $\mathrm{Fe} / \mathrm{O}$ ratios of $\sim 0.1$ with $\mathrm{Fe}$ ionization states of $\sim 14$, had no measurable enhancements in the ${ }^{3} \mathrm{He} /{ }^{4} \mathrm{He}$ ratio, and were associated with type II bursts (Reames 1999; Cliver 2000).

Based on these early measurements, most researchers accepted the notion that CME-shock associated large gradual SEP and ESP events result from the diffusive shock acceleration (DSA) of thermal solar wind (Lee 1983; Tan et al. 1989), although others pointed out that the suprathermal tail of the solar wind may be the source (Gosling et al. 1981; Tsurutani and Lin 1985; Tan et al. 1989). Indeed, based on the correlation between the particle intensities, abundances, and energy spectra during ESP events and pre-event ion populations, Tsurutani and Lin (1985) and Tan et al. (1989) had suggested that the concomitant solar flares might provide the suprathermal seed particles accelerated at the IP shocks.

Since the mid-1990s, instruments with greater sensitivity and resolution on board Wind (Russell et al. 1995) and ACE (Stone et al. 1998) have provided major observational advances in terms of measuring the solar wind ion composition and its variations (Von Steiger et al. 2000) and comparing them with the energy-dependence and event-to-event variability of the ionic charge state, and elemental and isotopic composition in ESP and SEP events over a broad energy range (Oetliker et al. 1997; Mazur et al. 1999; Möbius et al. 1999; Cohen et al. 2005; Desai et al. 2006; Klecker et al. 2007). These new observations have made it possible to re-examine questions about the origin of the seed populations and improve understanding of how SEPs are accelerated and transported to $1 \mathrm{AU}$. The following subsections highlight major advances and insights into the origin, acceleration, and propagation of SEPs that have resulted from two decades of research. In particular, we present the state of knowledge of SEP studies by the end of 2014 and discuss open questions that are yet to be fully resolved.

\subsubsection{SEP origin}

Observations of extremely rare elements and rare tracer ions like impulsive SEP-associated ${ }^{3} \mathrm{He}$ and interstellar pickup $\mathrm{He}+$ ions in SEP and ESP events have provided compelling evidence that CME-driven shocks accelerate material preferentially out of a suprathermal "seed" population that comprises contributions from the heated solar wind, coronal material, and remnants of solar transient events (Mason et al. 1999; Gloeckler 2003; Desai et al. 2006; Mason et al. 2004; Allegrini et al. 2008; Dayeh et al. 2009). Other studies have shown that the abundances of heavy ions accelerated in SEP and ESP events are not well organized by any physical quantity such as the ion's Q/M ratio or its First Ionization Potential (FIP) when compared with the corresponding SW abundances (Mewaldt et al. 2002; Desai et al. 2003, 2006; Kahler et al. 2009). Further, Mewaldt et al. (2012a, b, c) found that the suprathermal Fe densities at $1 \mathrm{AU}$ are generally significantly greater one day before the occurrence of these large SEP events compared to all other days, perhaps indicating that the presence of high-density suprathermal $\mathrm{Fe}$ is necessary for SEP events with large Fe fluences to occur. Finally, Desai et al. (2003) found that the IP shock abundances were well correlated with the average abundances measured at the same energy $(\sim 1 \mathrm{MeV} /$ nucleon $)$ 

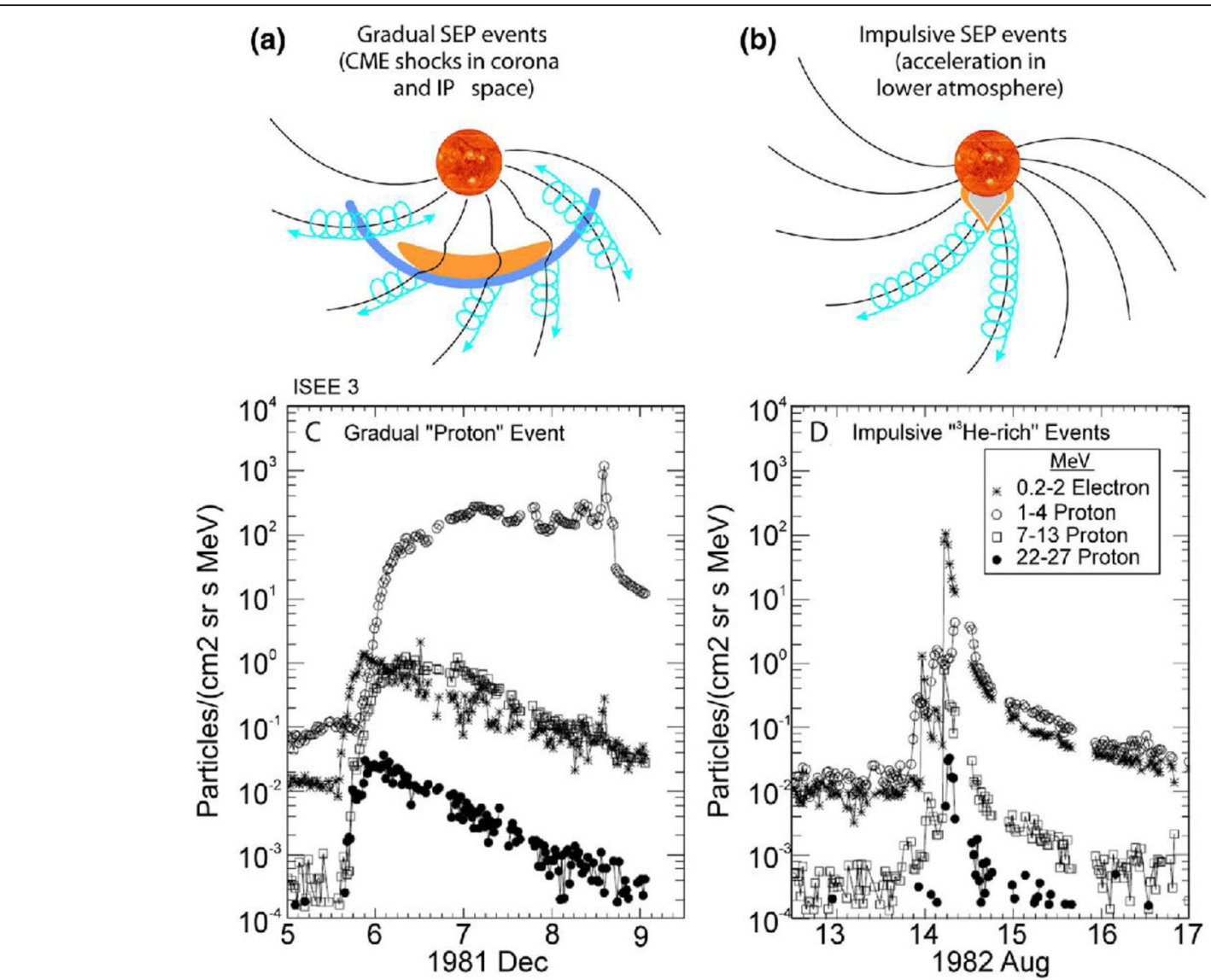

Fig. 19 The two-class paradigm for SEP events in which (a) gradual SEP events are produced as a result of diffusive acceleration by large-scale CME-driven coronal and interplanetary (IP) shock waves. The accelerated SEPs populate Interplanetary Magnetic Field (IMF) lines over a wide range of longitudes. (b) Impulsive SEP events are attributed to acceleration during magnetic reconnection in solar flares and observed when the observer is magnetically connected to the flare site. Intensity-time profiles of electrons and protons in (c) gradual and (d) impulsive SEP events. (Reproduced from Desai and Giacalone 2016, after Reames 1999)

in the interplanetary medium prior to the arrival of the IP shocks. In particular, elements with higher M/Q ratios are systematically depleted, which is consistent with shock acceleration models wherein ions with higher M/ $Q$ ratios are accelerated less efficiently than those with lower M/Q values (Lee 2005). Collectively, these results indicate that the material accelerated in large SEP events is quite distinct from that measured in the solar wind. Therefore, the SEP heavy ions are unlikely to originate from the bulk solar wind, but rather from a suprathermal tail that comprises ions from multiple sources, including ${ }^{3} \mathrm{He}$ and Fe-enriched material accelerated in flares and suprathermal material accelerated at previous CME shocks (Mason et al. 1999; Desai et al. 2006; Mewaldt et al. 2012a; Mewaldt et al. 2012b).

\subsubsection{SEP acceleration}

DSA comprises two main mechanisms, namely, shockdrift mechanism at quasi-perpendicular shocks (Decker 1981), and first-order Fermi mechanism at quasi-parallel shocks (Lee 1983). DSA theory successfully predicts some SEP observations, but fails to prevail as a universal theory in explaining most SEP events, partly because of external drivers that simultaneously affect the observed properties at $1 \mathrm{AU}$, including species-dependent escape from the IP shock, ambient turbulence, and shock finite size and geometry. For instance, SEP studies have shown that the differential energy spectra of $\mathrm{H}$-Fe nuclei in large SEP events exhibit a distinct form of a broken (i.e., double) power-law (hereafter PL) with a characteristic break-energy (hereafter $\mathbf{E}_{\mathbf{o}}$ ) (Tylka et al. 2005). In contrast, DSA theory predicts a single power-law. The location of $\mathbf{E}_{\mathbf{o}}$ was found to typically decrease for the heavier ion species as a power-law function of ion's charge-tomass ratio (Zank et al. 2000; Tylka et al. 2010; Mewaldt et al. 2012a). This systematic Q/M dependence occurs because the energy spectra roll-over or break at the same value of the diffusion coefficient for different species, which depends on ion rigidity or the $\mathrm{Q} / \mathrm{M}$ ratio (Tylka et al. 2000; Cohen et al. 2005; Mewaldt et al. 
2005). Li et al. 2009a) generalized an SEP acceleration model by including varying levels of turbulence near shocks of different obliquity and predicted that $\alpha$ could range between $\sim 0.2$ for weaker scattering near quasiperpendicular shocks and $\sim 2$ for stronger scattering near quasi-parallel shocks. Finally, Alfvén waves generated by energetic protons streaming upstream of ICME shocks could trap particles locally near the shock (Lario et al. 2005). This indicates that particle scattering and trapping near the shock, in some cases, could be dominated by a dynamic wave spectrum rather than a more universal background Kolmogorov-like wave spectrum (Tylka et al. 2005; Ng et al. 2003).

\subsubsection{SEP transport}

Effects of interplanetary transport on the temporal evolution of the heavy ion abundances and spectra are also believed to play a critical role in determining SEP observations at 1 AU. Tylka et al. (1999) and Ng et al. 1999, modeled the energy spectra and systematic temporal evolution of the elemental abundances of $\sim 5-10 \mathrm{MeV} /$ nucleon $\mathrm{He}, \mathrm{C}, \mathrm{O}, \mathrm{Ne}, \mathrm{Si}$, and $\mathrm{Fe}$ ions in two large SEP events in terms of rigidity-dependent trapping and scattering by Alfvén waves generated by streaming energetic protons accelerated at CME-driven shocks. These observations were successfully modeled using self-consistent numerical calculations of wave generation or amplification by shock-accelerated protons escaping or streaming away from the near-Sun CME shock (Lee 2005; Ng et al. 2003; Ng et al. 2012). Such self-excited Alfvén waves can scatter and trap particles near the shock and increase its acceleration efficiency. This, in turn, throttles the proton intensities near $\sim$ few $\mathrm{MeV} /$ nucleon resulting in energydependent upper bounds or plateaus, known as streaming limits (Reames 1990). Independently, Mason et al. (2006) pointed out that the dramatic variations in the temporal behavior of $\mathrm{Fe} / \mathrm{O}$ ratio at all energies between $\sim 0.1$ and $60 \mathrm{MeV} /$ nucleon vanish in $>70 \%$ of the prompt western hemisphere SEP events if the Fe intensities are compared to $\mathrm{O}$ intensities at $\sim$ twice the Fe kinetic energy-per-nucleon. To explore the physical process involved, Mason et al. (2012) modeled the rise phases in large SEP events and showed that the temporal evolution of $\mathrm{Fe} / \mathrm{O}$ can be reasonably fitted by a state-of-the-art model where the differences in the transport of Fe versus $\mathrm{O}$ are due to the slope of the turbulence spectrum of the IMF. Another effect of turbulence and waves which scatter SEPs can be a significant amount of transport perpendicular to the average magnetic field leading to wider angular particle spreads than the corresponding extent of their acceleration region (Dresing et al. 2012; Dröge et al. 2014; Dröge et al. 2016). However, the very widespread SEP events observed with the STEREO mission and close to Earth spacecraft (Gómez-Herrero et al.
2015; Lario et al. 2014, 2016) challenged state-of-the-art transport models based on the longitudinal distribution of electron anisotropies; Dresing et al. (2014) suggested that there exist different types of widespread events, on one hand, related to efficient perpendicular diffusion in the IP medium and, on the other hand, caused by an extended injection region close to the Sun.

In summary, observations from ACE, Wind, and STER EO during the 1995-2014 epoch have shown that large gradual SEP events are governed by a confluence of multiple processes and effects by the time they are observed at 1 AU. These include: (1) origin and variability of the suprathermal seed populations (Mason et al. 1999; Mason et al. 2005; Desai et al. 2003, 2004, 2006; Mewaldt et al. 2012c); (2) the efficiency with which populations from different sources and with distinct distribution functions are injected into the shock acceleration mechanisms; (3) factors that control the efficiency with which particles are accelerated (e.g., CME speed and kinetic energy, shock strength and obliquity (Kahler 2001; Kahler and Vourlidas 2013; Tylka et al. 2005; Mewaldt et al. 2008); (4) the presence or absence of multiple, interacting CMEs (Gopalswamy et al. 2004; Li et al. 2012) (5) the type, level, and characteristics of the waves and turbulence present near the shock and in the interplanetary medium (Tylka et al. 1999; Tylka et al. 2005; $\mathrm{Ng}$ et al. 1999; Cohen et al. 2003; Li et al. 2009a, b) and (6) the charge-to-mass $(\mathrm{Q} / \mathrm{M})$-dependence of scattering and transport through the turbulent interplanetary medium (G. M. Mason et al. 2006; Tylka et al. 2013). The relative roles of these effects continue to be topics of hot scientific debates, and unraveling their influence remains a major focus of SEP research. In addition, several studies claim a direct flare acceleration of the high-energy $(>25 \mathrm{MeV})$ proton component in large SEP events to augment that produced by coronal/ interplanetary shock waves driven by CMEs (Cane and Richardson 2003; Cane et al. 2006; Klein and Posner 2005; Aschwanden 2012).

In the following sections (6.3 and 6.4), we will highlight the progress made during the 2014-2019 timeframe and point out the key areas in which critical observations in the inner heliosphere from Parker Solar Probe and Solar Orbiter will advance our understanding of the physics of SEP events. These efforts are essential for developing models that can reliably forecast and mitigate radiation risks from extreme SEPs and are essential for deep space exploration.

\subsection{Progress in SEP observations during the VarSITI era (2015-2019) \\ 6.3.1 SEP origin}

Among the more unusual solar phenomena are the long-duration gamma-ray flares (LDGRFs). The prime 
characteristic of these events is delayed and prolonged $\gamma$-ray $(>100 \mathrm{MeV})$ emission after the impulsive phase (Ryan 2000). Recently, the Fermi Large Area Telescope (LAT) observed dozens of LDGRFs, with the most intense and longest duration example the 2012 March 7 event, for which $>100 \mathrm{MeV}$ emission was observed for nearly $20 \mathrm{hr}$ (Ajello et al. 2014). Share et al. (2018) characterized and cataloged 30 solar eruptive events observed by Fermi/LAT from 2008-2016, referring to this emission as "late-phase gamma-ray emission" (LPGRE). These authors produced and presented "light-bucket" time profiles for all the events, obtaining an estimate of the $>100 \mathrm{MeV} \gamma$-ray flux from within about $10^{\circ}$ of the Sun. Figure 20 presents an example of one of these time histories for the 2011 March 7 event, in which the LPGRE lasted 14 h. GBM and RHESSI observed impulsive hard X-rays up to only $100-300 \mathrm{keV}$ with no evidence for nuclear-line emission. The $>100 \mathrm{MeV}$ fluxes plotted in the inset reveal that the LPGRE started within minutes of the hard X-ray peak. It is clear from the rising LPGRE flux that it is due to a distinct particle acceleration phase and is not just the tail of emission from the impulsive phase of the flare.

In all the events studied, Share et al. (2018) found that the LPGRE is temporally and spectrally distinct from the impulsive phase emission. The spectra are consistent with the decay of pions produced by $>300 \mathrm{MeV}$ protons and are not consistent with primary electron bremsstrahlung with synchrotron losses. All but two of the LPGRE events were accompanied by a fast and broad CME. The LPGRE start times range from CME onset to $2 \mathrm{hr}$ later whereas their durations range from $\sim 0.1$ to 20 $\mathrm{hr}$ and appear to be correlated with the durations of the accompanying $>100 \mathrm{MeV}$ SEP proton events. Comparison showed that the number of $>500 \mathrm{MeV}$ protons producing the LPGRE is at least a factor of 10 larger than the number producing the impulsive phase $>100 \mathrm{MeV}$ $\gamma$-ray emission during the associated flare. The number of $>500 \mathrm{MeV}$ protons needed to produce the LPGRE ranges in nine events from $0.1 \%$ to $50 \%$ of the number of protons observed in the accompanying SEP event in interplanetary (IP) space (based on the observations by the GOES/HEPAD experiment and neutron monitors). There are significant systematic uncertainties in the SEP estimates, however.

One of the features of the LPGRE events Share et al. (2018) found is their association with fast CMEs and SEP production, which points to acceleration of particles by the shock produced by a fast CME as a clear candidate for the energy source for the $>300 \mathrm{MeV}$ protons that produces LPGRE. Furthermore, (Gopalswamy et al. 2018e) presented strong quantitative evidence that

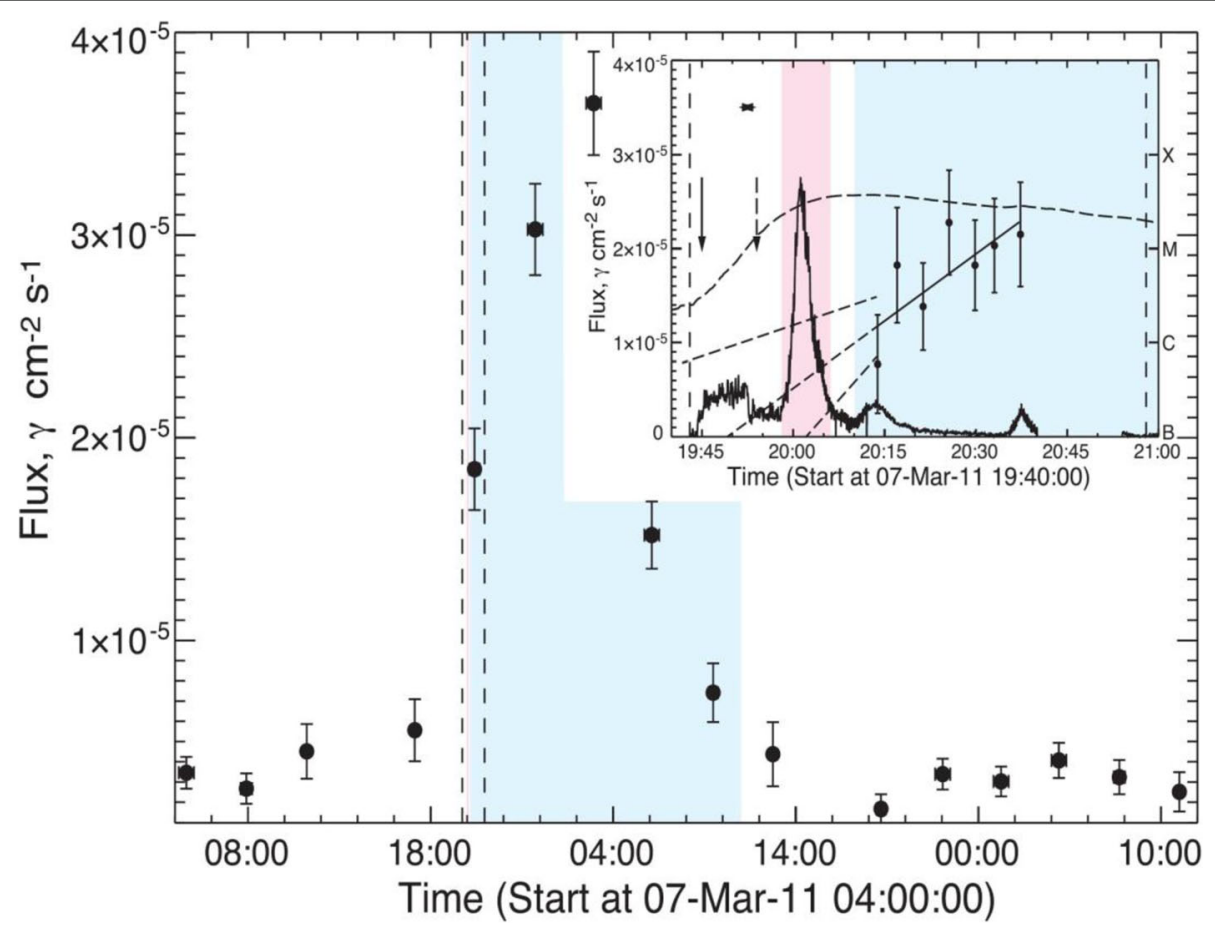

Fig. 20 Main plot: Time history of the $>100 \mathrm{MeV}$ light-bucket fluxes from $<10^{\circ}$ of the Sun, revealing LPGRE from the 2011 March 7 solar eruptive event. Vertical dashed lines show the GOES 1-8 ^ start and end times. Inset: 4 min accumulation light-bucket fluxes as detected by Fermi/LAT are shown. The dashed curve shows the GOES time history. The blue shaded region denotes the estimated duration of the LPGRE. The pink shaded region depicts where estimates of the flux of $>100 \mathrm{MeV}$ impulsive-flare $\gamma$-ray emission were made (see text; after Share et al. 2018) 
interplanetary type II radio bursts and such sustained gamma-ray emission (SGRE) events from the Sun are closely related. Out of the 30 SGRE events reported by (Share et al. 2018), they considered 13 events that had a duration exceeding $\sim 5 \mathrm{hr}$, thus excluding any flareimpulsive phase gamma-rays. These authors found that the SGRE duration has a linear relation with the ending frequency of the bursts (Fig. 21). The synchronism also found between the ending times of SGRE and the type II emission (Fig. 21) strongly supports the idea that the same shock accelerates electrons to produce type II bursts and protons $(>300 \mathrm{MeV}$ ) that propagate from the shock to the solar surface to produce SGRE via pion decay. A CME-shock origin could also explain the wide range of delays observed in LPGRE onset times: short LPGRE onset delays represent shock acceleration low in the corona (Gopalswamy et al. 2018c), while long LPGRE onset delays indicate that the CME had to expand over several solar radii before accelerating $>300$ $\mathrm{MeV}$ protons that could return to the Sun. The smooth time histories of the long duration LPGRE events can be explained by the precipitation of particles that are magnetically trapped in a reservoir (Reames 2013) behind the expanding CME.

Quantitative estimates, however, have indicated that only a very small fraction of accelerated protons can return to interact in the chromosphere (Hudson 2018; Klein et al. 2018) due to the transport of the protons back to the Sun against the magnetic mirror force, well after the flare when the CME is many solar radii above the surface with magnetic field strengths much lower than at the solar surface. Share et al. (2018) go around this difficulty by assuming that there is significant MHD turbulence on the field lines connecting the CME to the Sun, such as required in the model of (Ryan and Lee 1991). Furthermore, Kocharov et al. (2015) presented a shock-wave model and their estimated ratio of the number of protons that return to the Sun and interact to the number that escape into IP space depending on the amount of turbulence is consistent with the range estimated by Share et al. (2018) in their comparison of the number of $>500 \mathrm{MeV}$ protons producing LPGRE and those detected as SEPs in space. A variant on the magnetic trap model was proposed by Hudson (2018) and called the 'lasso' model in which the SEP particle accelerator crossed both open (SEP) and closed (LPGRE) field lines, leaving energetic particles on both.

de Nolfo et al. (2019) compared the total number of > $500 \mathrm{MeV}$ protons at $1 \mathrm{AU}$ by combining Payload for Matter-Antimatter Exploration and Light Nuclei Astrophysics (PAMELA) and STEREO spacecraft data with the number of high-energy protons at the Sun as deduced from Fermi/LAT (Share et al. 2018). Their analysis showed that the two proton numbers are uncorrelated such that their ratio spans more than 5 orders of magnitude, suggesting that the back precipitation of particles accelerated at CME-driven shocks is unlikely to be the source of the LPGRE emission. They discussed an alternative explanation for LPGREs based on continuous particle acceleration and trapping within large coronal structures that are not causally connected to the CME shock, within the context of new remote observations of these loops available.

\subsubsection{SEP acceleration}

A lively debate has continued in recent years on the question of the principal source of high-energy protons in large SEP events. Some studies have provided new support for a significant contributory or dominant role for flare acceleration of high-energy protons in gradual SEP events, contrary to the generally accepted scenario favoring shock acceleration. This new evidence is mainly
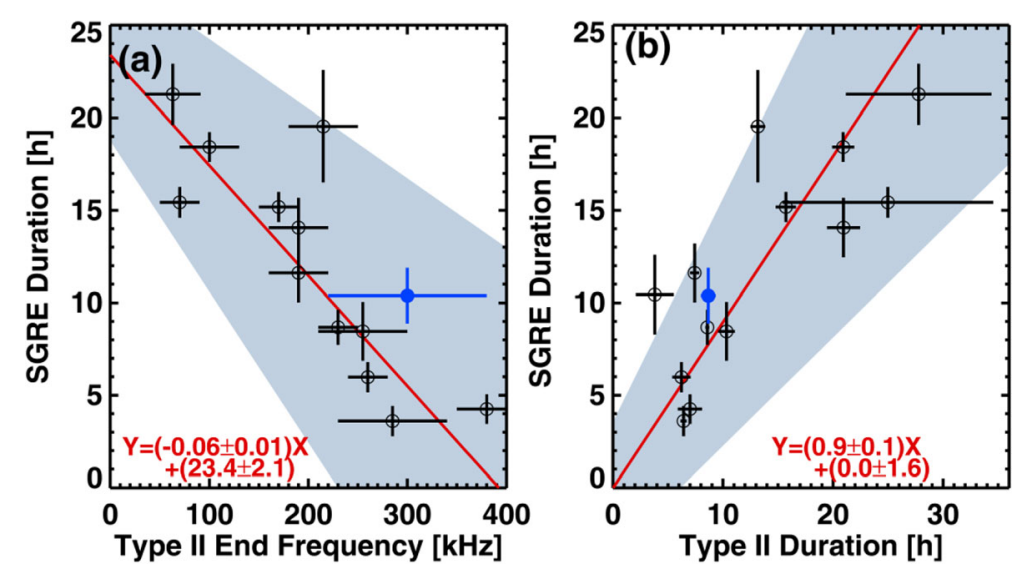

Fig. 21 Scatter plots of SGRE duration with type II ending frequency (a) and type II duration (b). The best-fit lines (red) are obtained using the Orthogonal Distance Regression method, which considers errors in both X and $Y$ variables. The shaded area represents $95 \%$ confidence interval of the fit (after Gopalswamy et al. (2018c)) 
based on correlations between the sizes of X-ray and/or microwave bursts and the SEP fluence at different energies, e.g., for 15-40 MeV protons (Trottet et al. 2015), > $30 \mathrm{MeV}$ protons (Le and Zhang 2017), > $50 \mathrm{MeV}$ protons (Dierckxsens et al. 2015), and > $100 \mathrm{MeV}$ protons (Grechnev et al. 2015; Le et al. 2017). In order to assess the above correlations, the technique of partial correlation coefficients (see Trottet et al. (2015) for a detailed description) has been used along with the classical Pearson correlation coefficient, to remove the correlation effects between the solar parameters themselves. In particular, Grechnev et al. (2015) addressed the relation between the $>100 \mathrm{MeV}$ proton fluences measured by the Geostationary operational Environmental Satellite (GOES) monitors and the associated flare microwave fluences at $35 \mathrm{GHz}$, recorded by the Nobeyama Radio Polarimeters, over the 1996-2014 time interval. Grechnev et al. (2015) found a partial correlation coefficient of 0.67 , versus 0.001 for a corresponding comparison of the $>100 \mathrm{MeV}$ proton fluence and the CME speed, concluding that these SEP events originated in the associated flares. These results were criticized by Cliver (2016), according to which the exclusion in Grechnev et al. (2015) of four outlying "abundant proton" events (black squares in the orange rectangle in Fig. 22) is not justified if one considers the associated CME speeds and widths, and electron-to-proton ratios which are comparable to those in the main sequence (black circles in Fig. 22). On the contrary, inclusion of such events in the analysis reverses the conclusion in favor of shock acceleration for the > $100 \mathrm{MeV}$ protons. Nevertheless, we point out these results are based on the assumptions that non-DH -associated SEPs are flare generated and those behind the limb are shock generated, which although reasonable are not ultimately proven.

Several other recent studies support the prevailing shock picture for gradual SEP events, such as the observation of a prompt SEP event at widespread locations in conjunction with the longitudinal propagation of a white-light shock (Lario et al. 2016), the SEP source temperatures (Reames 2015) and the hierarchical relationship found between the fluence spectra of gradual SEP events and the kinematics of the CMEs (Gopalswamy et al. 2017a). Gopalswamy et al. (2017c) analyzed the SEP fluence spectra of three classes of SEP events (Filament eruption (FE) SEP events, well-connected regular ones, and SEP/GLEs) over cycles 23 and 24. They found that: FE SEP events have the softest spectra and lowest initial acceleration; SEP/GLE events have the hardest spectra and the largest initial acceleration; the regular SEP events have intermediate spectral indices and acceleration. It has to be noted that the computed spectral indices by considering a simple power law, without taking into account possible rollovers and breaks,

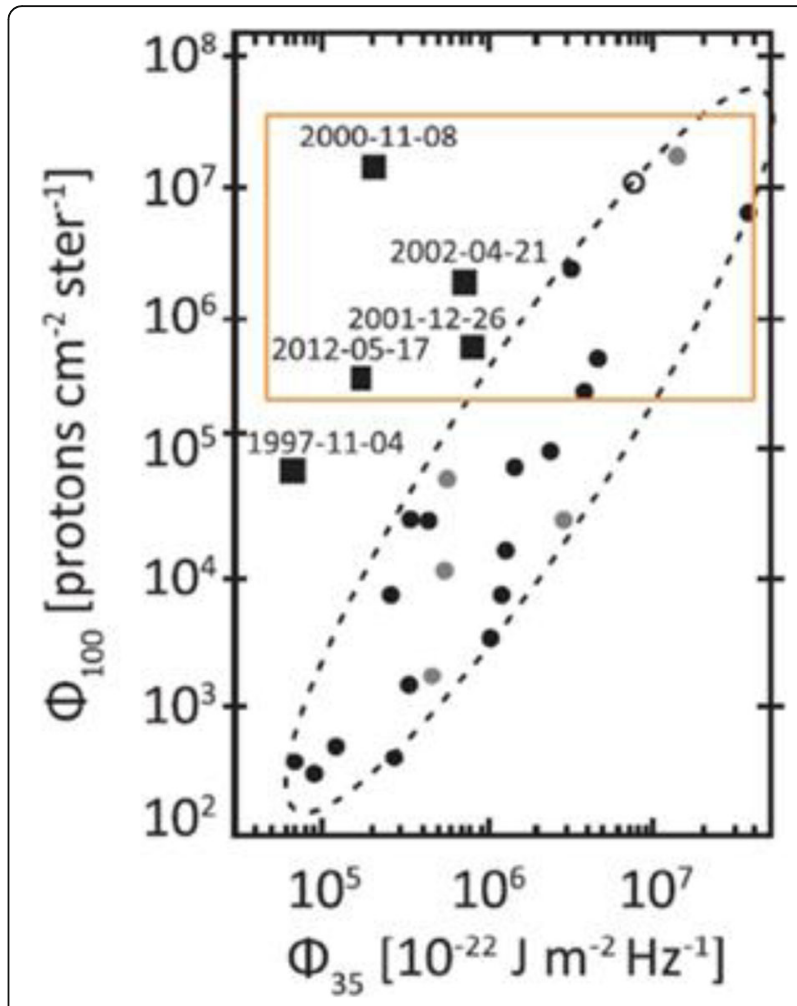

Fig. 22 Scatterplot of longitude-corrected > $100 \mathrm{MeV}$ proton fluence (\$100) vs. $35 \mathrm{GHz}$ fluence (\$35) for solar proton events from 1996 to 2014; black circles and squares (W21-W90); gray circles (E30-W20); open circles (< E30). The orange rectangle isolates events with $\Phi 100>2 \times 10^{5}$ pfu (from Cliver 2016)

thus mainly representing the spectrum behavior at lower energies. The hierarchical relationship was shown to be present (Gopalswamy et al. 2017a) also in terms of the average starting frequencies of the associated type II bursts and the shock formation height (as obtained by matching the onset time of the type II bursts with the CME leading edge height-time history). Such behavior could be explained by considering that the rapid acceleration of CMEs leads to very high initial speeds and hence a shock formation close to the Sun, where the ambient magnetic field and density are high for an efficient particle acceleration, resulting in harder spectra for GLE events.

Understanding the origin of SEP/GLE events is especially challenging, as particles are accelerated up to $\mathrm{GeV}$ energies and can reach the Earth's atmosphere in about $10 \mathrm{~min}$, while the various acceleration mechanisms may be expected to exhibit different characteristic timescales. The comparison between the solar particle release (SPR) time and the onset time of the SEP event associated solar phenomena is generally used to pose temporal constraints that any putative acceleration process must meet. Nevertheless, the different methodologies used to perform such studies and underlying assumptions, e.g., 
the constant SPR at all energies and the particle's scatter-free propagation in the interplanetary space, e.g., questioned by Wang and Qin (2015), can lead to contrasting results about the principal source of particle acceleration. The most recent SEP/GLE event, which occurred on 2017 September 10, has been attributed to both flare and shock acceleration, e.g., by Zhao et al. (2018) and Gopalswamy et al. (2018c), respectively, due to their different evaluations of the SPR and the Type II bursts onset time. In particular Gopalswamy et al. (2018c), confirmed the parabolic relationship between the eruption longitude and the CME height at SPR, which is considered to be the key to the understanding of particle acceleration by shocks and the magnetic connectivity to the observer. Those authors also showed that the 2017 September 10 GLE did not have an unusually hard spectrum (Schwadron et al. 2018), but a softerthan-average spectrum for a GLE event, having the 10$100 \mathrm{MeV}$ fluence spectral index of 3.17 with respect to the average one of 2.68 for SEP/GLEs (Gopalswamy et al. 2016). They suggested this to be due to the poor longitudinal and latitudinal connectivity of the shock nose with the Earth, possibly compounded by the weak ambient magnetic field reducing the shock acceleration efficiency. Similarly, it is suggested that some gradual SEP events with high initial speeds did not produce a GLE, as only the shock flanks were magnetically connected with the Earth (Gopalswamy et al. 2018c). Nevertheless, this has to be reconciled with the hypothesis that highenergy protons are accelerated preferentially by quasiperpendicular shocks which could be located at the shock flanks (Schwadron et al. 2015; Kong et al. 2019), as will be discussed in sub-section 6.3.2.

\subsubsection{SEP transport}

The unprecedented orbits of the two-spacecraft STER EO mission provided well-separated observations at 1 AU and allowed to study the longitudinal distribution of SEPs and especially events with extraordinarily wide particle spreads in great detail (Lario et al. 2014, 2016; Gómez-Herrero et al. 2015; Nina Dresing et al. 2018). Based on Gaussian functions applied to multi-spacecraft events, Lario et al. (2013) and Richardson et al. (2014) determined their mean widths to be between $36^{\circ}$ (27-37 $\mathrm{MeV}$ protons) and $49^{\circ}$ (71-112 keV electrons). However, a large event to event variation was observed which also limited the determination of the average displacement of the Gaussian center with respect to the longitude of the associated flare site. However, the displacements towards the west of the flare site found in the above studies may be caused by the associated CME-driven shocks that would shift the Gaussian distributions towards the central meridian as viewed from the spacecraft.
In a similar manner, Cohen et al. (2017) have systematically investigated the energy and $\mathrm{Q} / \mathrm{M}$ dependence of the longitudinal distributions for large ion events using STEREO and close-to-Earth spacecraft reporting comparable values for widths and mean Gaussian-center displacements. While the widths were found to show an energy dependence with distributions narrowing with increasing energy, no $\mathrm{Q} / \mathrm{M}$ dependence to the widths of Gaussian centers were found for the 41 ion event distributions studied (see Fig. 23). This suggested that lower energy ions might experience more field line co-rotation, or are accelerated over a larger portion of the CMEdriven shock or for longer times as the shock expands. Rigidity-related processes seemed, however, not to be important in terms of longitudinal spreading of the particles.

The STEREO mission has also enabled the identification and study of some extreme cases of widespread events with distributions up to $360^{\circ}$ around the Sun (Dresing et al. 2012; Dresing et al. 2014; Lario et al. 2014, 2016; Gómez-Herrero et al. 2015). However, even when the application of interplanetary transport models (see Section 6.3.2) suggests the presence of strong perpendicular transport (Dröge et al. 2014; Dröge et al. 2016; Strauss et al. 2017), it seems that all widespread events need at least a somewhat extended injection region of e.g., $25^{\circ}$ width (Strauss et al. 2017). The nature of this extended injection region may be an extended shock which accelerates and injects the particles over a wide longitudinal range. Modern reconstruction techniques of the coronal and interplanetary shock using EUV and white-light data (see section 2.2) have shown a good agreement between the inferred SEP injection times and the times of the expanding shock intersecting the magnetic footpoint of the spacecraft (Kouloumvakos et al. 2016; Lario et al. 2016). For well-connected observers, similar correlations were found also for the time when the associated EUV wave intersects the spacecraft magnetic footpoint in the low corona and the role of these waves in terms of particle acceleration and coronal transport were discussed controversially (Park et al. 2013; Lario et al. 2014; Miteva et al. 2014). In the case of the farthest separated spacecraft in extreme widespread events, however, the event can usually not solely be explained by the established magnetic connection to the shock or an EUV wave but transport effects are likely to play a role in spreading the SEPs to the farthest observers (Lario et al. 2014; A Kouloumvakos et al. 2016). While shocks are accepted to be the main source of large and gradual solar ion events, their role for efficient electron acceleration is still under debate (Dresing et al. 2020 and references therein). Alternative scenarios providing wide injection regions involved in widespread electron events (Dresing et al. 2014) are the presence of 

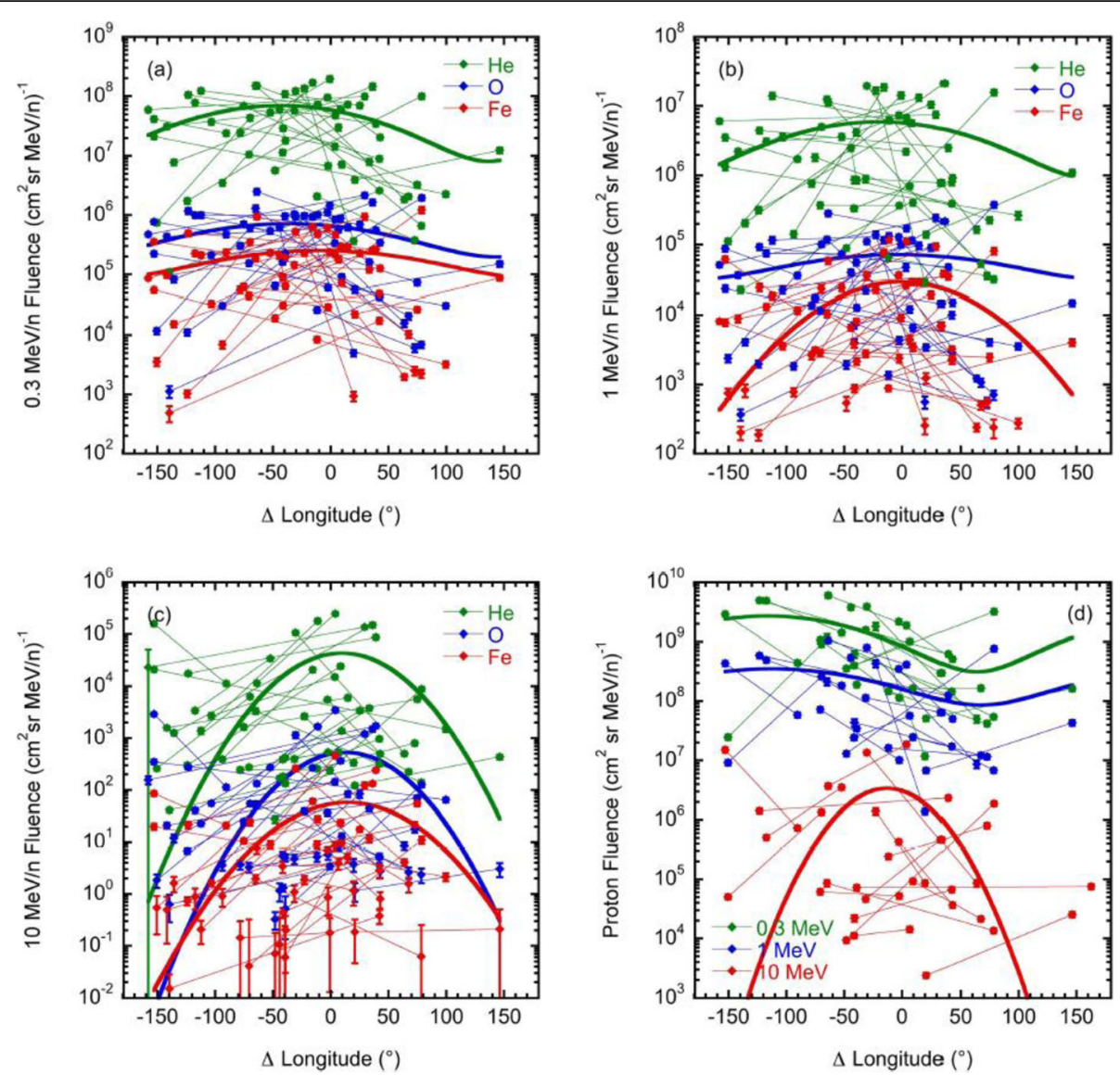

Fig. 23 Peak intensities of He, $\mathrm{O}$, and Fe as a function of flare-spacecraft footpoint separations ( $\Delta$ Longitude) of two-spacecraft events (Cohen et al. 2017). Negative values of $\Delta$ Longitude correspond to footpoints west of the flare location, and positive values correspond to locations east of the flare. The different panels show different energies with (a) 0.3 , (b) 1 , and (c) $10 \mathrm{MeV} / \mathrm{n}$. Panel (d) shows protons at all three energies.

Individual events are connected by lines, the thick curves show periodic Gaussian fits

fan-shaped magnetic field lines (Klein et al. 2008), but non-uniform or non-radial spatial injections (Klassen et al. 2016, 2018) may play a role as well.

The 26 Dec 2013 widespread electron event (Dresing et al. 2018) suggested to be caused by a shock, at a first glance, because of its wide SEP spread and very longlasting proton and electron anisotropies pointing to a time-extended injection different to a flare. However, other features of the event, like an additional highenergy SEP component, arriving four hours later than the first one, could not be explained solely by the presence of a shock and these authors suggested a trapping scenario to be involved in forming the characteristics of the event. Additionally, to accelerating SEPs, CMEs can also play an important role in SEP transport when they have propagated into interplanetary space. If a previous CME, which is still magnetically anchored at the Sun, is convected over the observer just at the time when another eruptive event occurs at the Sun, new SEPs may be injected into this ICME and propagate through it.
The magnetic connection of an observer inside this structure to the Sun is dramatically changed and one loop leg may even provide otherwise unlikely magnetic connections to eastern longitudes so that SEPs from these source regions can be observed even if the source extent is small (Richardson et al. 1991). First arriving SEPs may then also arrive in anti-sunward pointing telescopes (Gómez-Herrero et al. 2017).

Measuring an SEP event inside an ICME confirms not only that the structure is still magnetically anchored at the Sun (Malandraki et al. 2002, 2005) but also that solar energetic electron observations constitute a tool to probe the magnetic structure, e.g., the winding of the magnetic field inside the ICME (Kahler et al. 2011; Tan et al. 2012). If combined with reconstruction of the early CME based on coronagraph observations, one can then determine the dimension of the large-scale structure, such as its loop length, at $1 \mathrm{AU}$, i.e., for times when it propagated far out of the fields of view of coronagraphs (Dresing et al. 2016). 


\subsubsection{SEP compositional results}

Measurements of relative abundances of heavy elements, their isotopic and ionic charge state composition in SEP events have been used in a wide variety of ways to infer critical information about the origins of the seed populations and the physical conditions under which these populations are produced, and the manner in which these seed particles are accelerated by CME shocks or in solar jets. In addition, we can infer the conditions that affect their transport through the solar corona, the interplanetary medium, and out into the heliosphere (Reames 2016; Desai et al. 2016a). For instance, it is well established that elemental abundances in SEPs exhibit the so-called first ionization potential or FIP effect when compared with corresponding photospheric abundances, as shown in Fig. 24. Based on well-documented differences between the average SEP abundances of elements such as $C, S$, and $P$ that have intermediate FIP values (Desai et al. 2006; Reames 2018) and those measured in the slow solar wind (Bochsler 2008), Reames (2018) suggested that these differences could be due to the action of ponderomotive forces of Alfvén waves on $\mathrm{C}, \mathrm{S}$, and $\mathrm{P}$ ions on open field lines and their corresponding absence on neutral $\mathrm{C}, \mathrm{S}$, and $\mathrm{P}$ atoms on closed field lines in the chromosphere (Laming 2015). According to this hypothesis, coronal material accelerated in SEPs differs from that found in the solar wind (see Fig. 25), because the chromospheric plasma that later becomes SEPs enters the corona on closed field lines in active regions, while the corresponding plasma that later becomes the solar wind appears on open field lines, thus resulting in the observed differences between the $\mathrm{C}, \mathrm{S}$, and $\mathrm{P}$ abundances in SEPs and the SW (Reames 2018, 2020).

Numerous SEP studies have also shown that the differential energy spectra of $\mathrm{H}$-Fe nuclei in large SEP events exhibit a distinct form of a broken (i.e., double) powerlaw (hereafter PL) with a characteristic break-energy (hereafter $\boldsymbol{E}_{\boldsymbol{o}}$ ). Desai et al. 2016a, b surveyed the heavy ion spectra in 46 isolated, large gradual SEP events observed in solar cycles 23 and 24, and found that the $\mathrm{Fe}$ spectra had lower $\boldsymbol{E}_{\boldsymbol{o}}$ owing to the lower Q/M ratio or higher rigidity of Fe when compared with $\mathrm{O}$. Figure 26, taken from (Desai et al., b), shows an example of this relation. This systematic $\mathrm{Q} / \mathrm{M}$ dependence occurs because the energy spectra roll-over or break at the same value of the diffusion coefficient for different species, which depends on ion rigidity or the $\mathrm{Q} / \mathrm{M}$ ratio (Tylka et al. 2000; Cohen et al. 2005; Mewaldt et al. 2005; Desai et al.
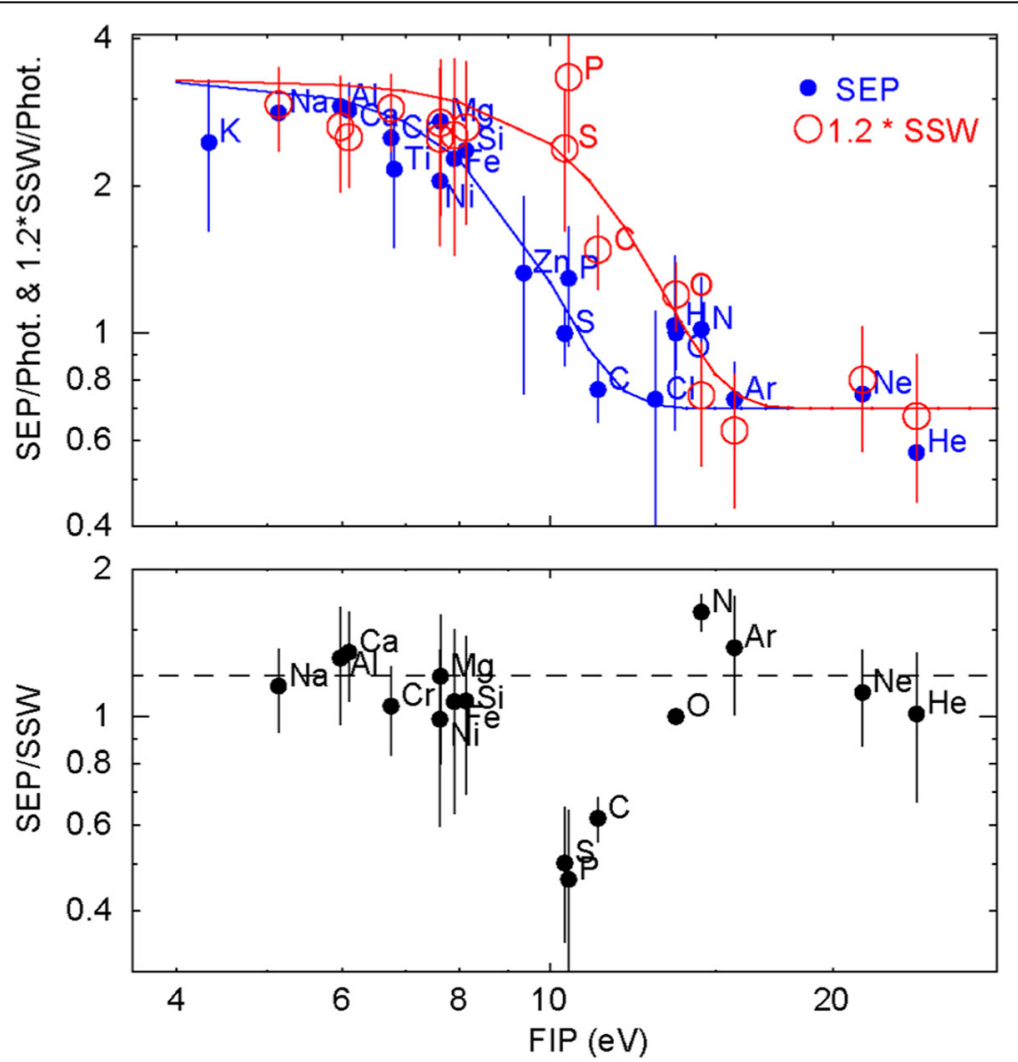

Fig. 24 The upper panel shows the SEP/photospheric and 1.2xslow solar wind (SSW)/photospheric abundance ratios as a function of FIP. Curves help show the trends of each data set. The lower panel shows the ratio of the "coronal" abundances from SEPs to those of the slow SW (Bochsler 2008), as a function of FIP. The dashed line suggests the preferred normalization factor of 1.2. Adopted from Reames (2018) 


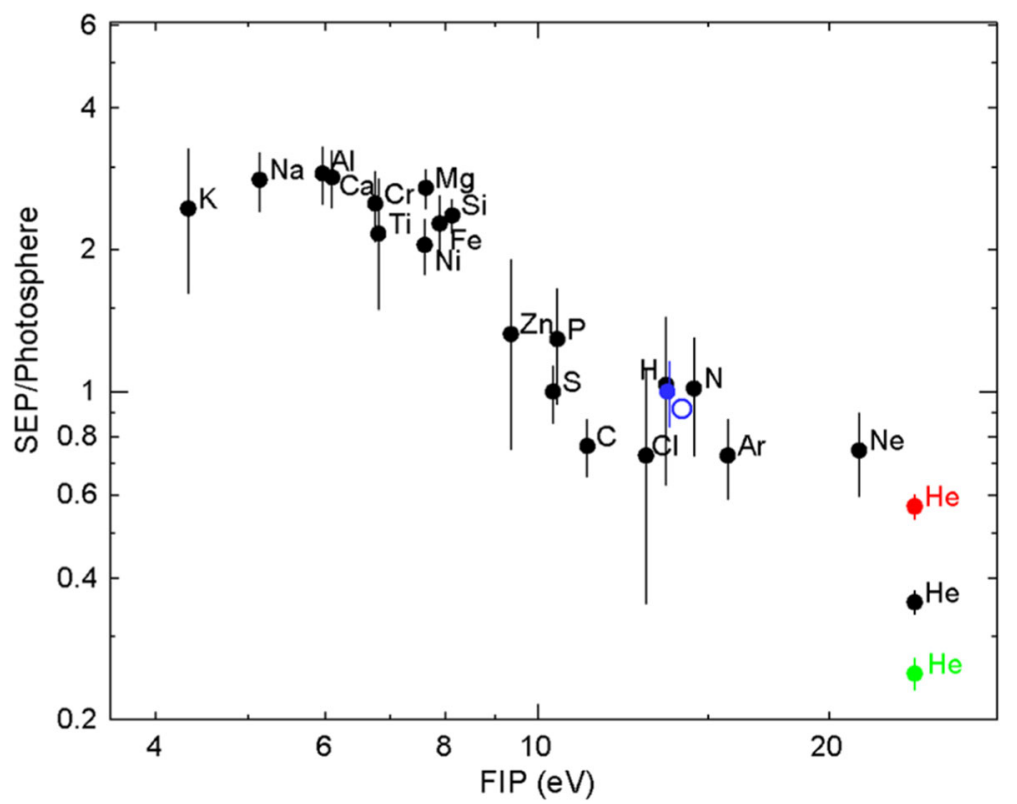

Fig. 25 SEP abundances (from Reames 2014) divided by photospheric abundances (from Reames 2014) divided by photospheric abundances (from Caffau et al. 2011, Lodders et al. 2009), normalized at O (blue). Abundances of He in three SEP events are also shown (taken from Reames 2018)

$2016 \mathrm{a}, \mathrm{b})$. The authors also found that $\alpha$ varies between $\sim 0.2$ and 3, where extreme SEP events associated with Ground Level Enhancements (GLE) often exceeded the upper limit of 2 (see Fig. 26), as expected from theoretical predictions ( $\mathrm{Li}$ et al. 2009a).

Later, Zhao et al. 2016a, b performed a comprehensive numerical simulation study and found that the single power law spectrum near the Sun transitions into a double power law near $1 \mathrm{AU}$. The authors found that the spectral indices above and below $\boldsymbol{E}_{\boldsymbol{o}}$, along with the value of $\boldsymbol{E}_{\boldsymbol{o}}$, are related to the Kolmogorov-like interplanetary magnetic turbulence spectrum. Zhao et al. 2016a, b also studied the proton energy spectra in the decay phase (reservoir) of selected SEP events, where transport effects are expected to be minimal. They found that some events (see Fig. 27) in which the event-integrated spectra were described by double power-laws transitioned into a single power-law during the reservoir or decay phase. This behavior was interpreted in terms of scattering, streaming, and diffusion effects for lower particles below $\boldsymbol{E}_{\boldsymbol{o}}$

Finally, Zelina et al. (2017) and Doran et al. (2019) suggested that the observed temporal evolution of heavy
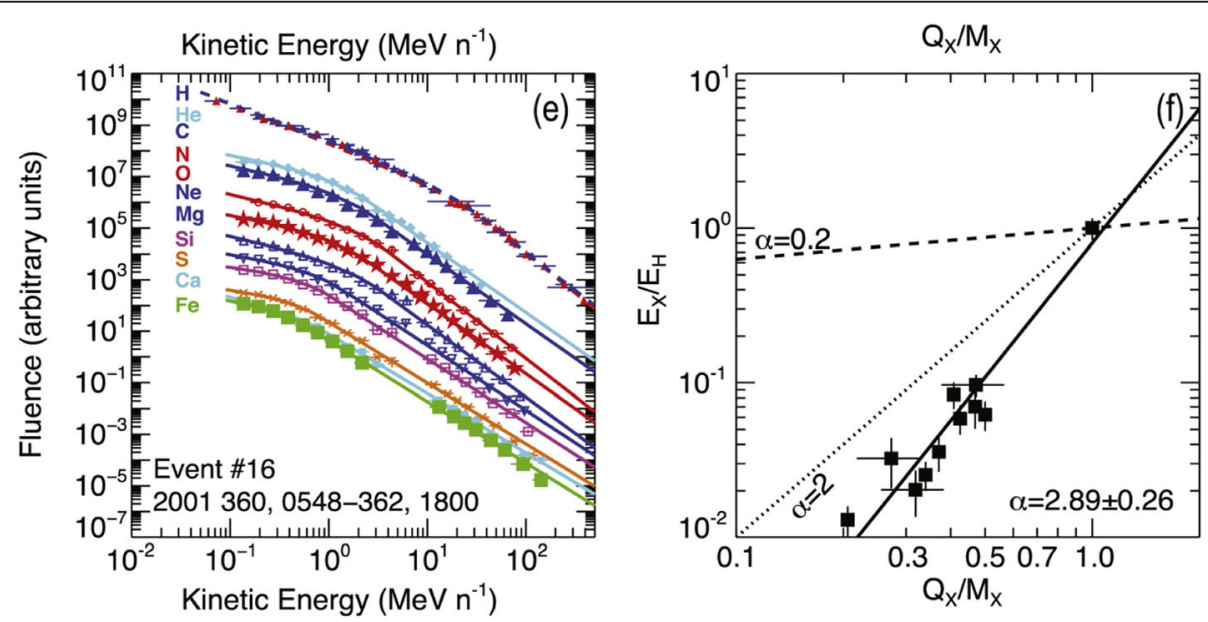

Fig. 26 a Event-integrated differential fluences vs. energy of $\sim 0.1-500 \mathrm{MeV} /$ nucleon for H-Fe nuclei during a large SEP event, taken from Desai et al. (2016b). The energy spectra for different species are offset for clarity. Solid lines are Band function fits to the spectra. $\mathbf{b}$ Spectral break energy $E_{X}$ of species $X$ normalized to $E_{H}$-break energy of $H$ vs. the ion's charge-to-mass $(Q / M)$ ratio. Solid lines are fits to the data $E_{X} / E_{H}=n o$ $(\mathrm{Qx} / \mathrm{Mx})^{a}$; dashed line: same equation with $a=2$; dotted line: same equation with $a=0.2$ 


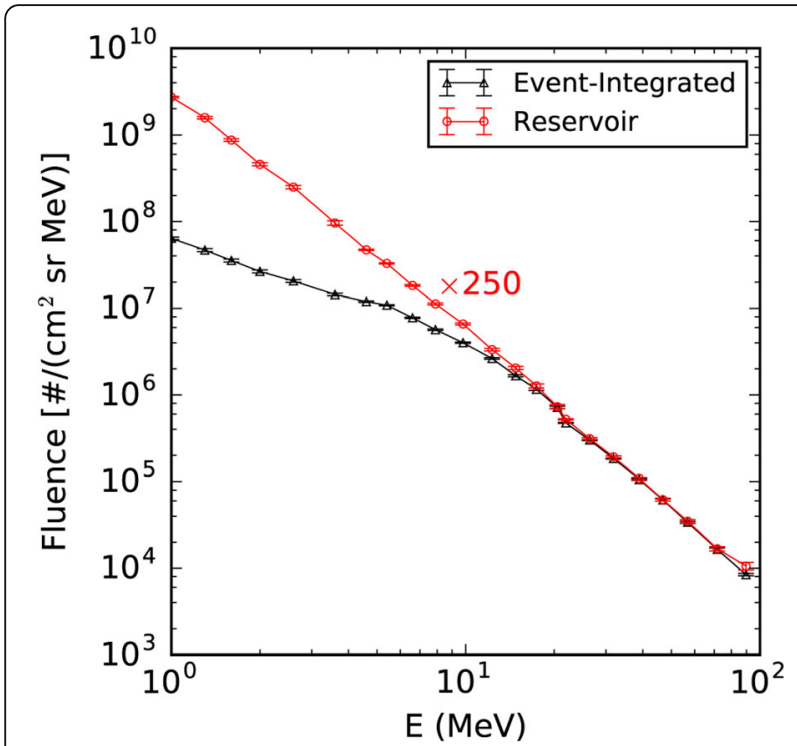

Fig. 27 Event integrated and reservoir spectra during the 11/22/ 1977 SEP event. The spectrum shows a double power-law feature, while the reservoir spectrum exhibits a single power law (from Zhao et al. 2017). This effect was interpreted in terms of particle streaming and rigidity-dependent scattering effects that modify the spectrum at lower energies

ion elemental abundances and energy spectra during some large SEP events observed at ACE and STEREO could be accounted either by rigidity-dependent scattering (Parker 1965; Mason et al. 2006; Mason et al. 2012; Tylka et al. 2013) (or via drift-associated Q/Mdependent deceleration during transport through the interplanetary medium (Kota 1979; Dalla et al. 2015).

To reconcile the puzzling SEP observations from Wind, ACE, and STEREO with the two-class paradigm of the mid-1990's, Reames (2020) has recently proposed that the SEPs observed at 1 AU (see Fig. 28) can be grouped into four sub-classes based on where and how they are accelerated, namely (1) Pure impulsive SEP events are produced when the $\sim 3 \mathrm{MK}$ coronal plasma is accelerated by magnetic reconnection islands in solar jets around $\sim 1.5$ Rs. These events have large M/Qdependent enhancements for $\mathrm{H}$ through ultra-heavy ions as well as the ${ }^{3} \mathrm{He} /{ }^{4} \mathrm{He}$ ratio; (2) Impulsive SEP events are produced when CMEs from the same jets are fast enough to drive shocks. These shocks re-accelerate the $\sim 3 \mathrm{MK}$ impulsive suprathermal material mixed with ambient plasma mainly comprising protons; (3) Weak gradual SEP events occur when wide, moderately fast (> $500 \mathrm{~km} / \mathrm{s}$ ) CME-driven shock waves accelerate the ambient coronal plasma, including protons, but also preferentially accelerate the faster impulsive $\sim 3 \mathrm{MK}$ suprathermal heavy ion material left over in the corona from many small jets; and (4) Strong gradual SEP events are produced when wide, fast $(>1000 \mathrm{~km} / \mathrm{s}$ )
CME-driven shock waves predominantly accelerate material from the ambient $\sim 1-2 \mathrm{MK}$ coronal plasma at $\sim 2-3 R_{\mathrm{S}}$. These events have negligible amounts of impulsive suprathermal ions.

\subsection{Progress in SEP theory and modeling during the VarSITI era (2015-2019) \\ 6.4.1 SEP acceleration}

Valuable insight on particle acceleration at coronal shock waves has been recently obtained by studying the evolution of CME and shocks low in the corona, as well as their interaction with underlying magnetic fields and coronal plasma, both through data-driven or analytical modeling, as well as MHD simulations and combinations of different approaches. New information has been gathered about the relevant parameters for efficient shock acceleration (such as the Mach number, compression ratios, geometry of shock waves), the primary acceleration regions along the shock, the role of coronal magnetic field configuration and how these factors are related to the particle spectra observed in space.

Forward modeling techniques (Rouillard et al. 2016; Salas-Matamoros et al. 2016; Kouloumvakos et al. 2019) have been used to perform the geometrical fitting of shock waves or CMEs with different geometrical models (i.e., the spheroid or the graduated cylindrical model), based on multipoint imaging. Rouillard et al. 2016 performed a triangulation of the three-dimensional (3D) expansion of high-pressure fronts using three simultaneous viewpoints from SOHO, SDO, and STEREO observations for the 17 May 2012 GLE event. In conjunction, they inverted remote-sensing observations to derive the background coronal conditions through which the pressure front propagates and modeled the topology of the background magnetic field. To this end, they employed both the PFSS model, based on the line of sight component measured by HMI, and the MHD MAST (Magnetohydrodynamic Around a Sphere Thermodynamic) model with improved thermodynamics including realistic energy equations with thermal conduction parallel to the magnetic field, radiative losses, and coronal heating (Lionello et al. 2008). They derived the normal speed and the Mach number $\left(M_{\mathrm{fm}}\right)$ over the entire surface of the CME front, as well as the shock geometry and the magnetic connectivity of the near-Earth environment with the shock. A band of high $M_{\text {fm }}$ values was found to be co-located with the region of quasi-perpendicular geometry, which evolved within $10 \mathrm{~min}$ into a quasiparallel geometry, reaching its highest values near the nose of the CME. In addition, a super-critical shock $\left(M_{\mathrm{fm}}\right.$ values in excess of (3) had formed at the release time of high-energy particles, suggesting that delayed release times of $\mathrm{GeV}$ protons could be related with the time needed for the shock to become super-critical. 


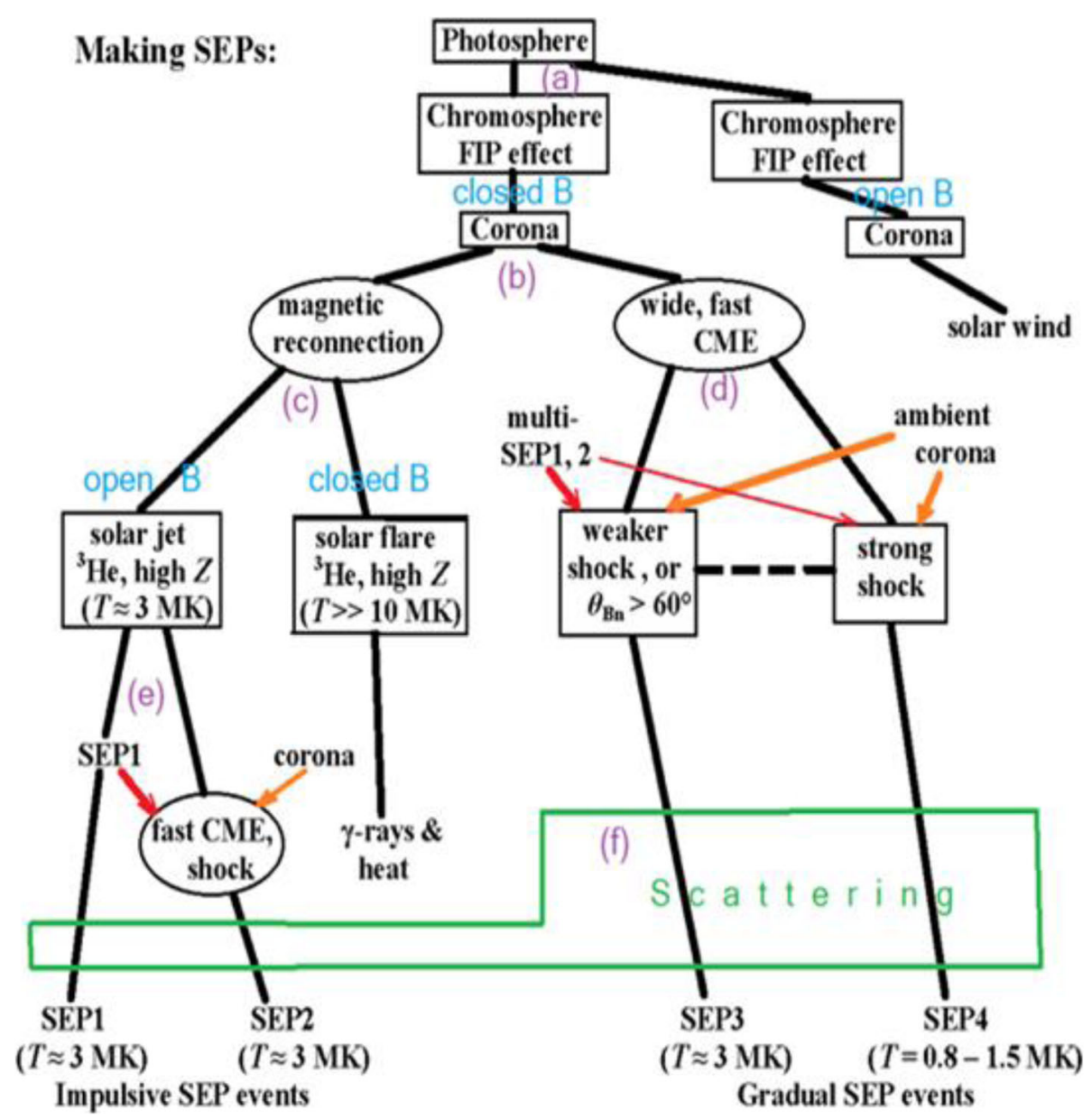

Fig. 28 Possible reconciliation between the two-class paradigm of the early 1900s and the puzzling SEP observations from Wind, ACE, and STER EO. The SEPs are grouped into four sub-classes according to their acceleration locations and the contributions of relevant physical processes (taken from Reames 2020). a Elements experience different FIP processing on open and closed field lines. b SEPs are accelerated in magnetic reconnection regions or by CME shocks. c SEPs enriched in ${ }^{3} \mathrm{He}$ and heavy ions escape along open field lines associated with solar jets, and not from closed field lines associated with solar flares. $\mathbf{d}$ Weaker CME-driven shocks preferentially accelerate residual suprathermal material left over from multiple jets, while the stronger CME shocks accelerate ambient coronal material. e Fast CMEs associated with jets also accelerate suprathermal jet-accelerated material. f Rigidity-dependent trapping near CME shocks or scattering in the corona and the IP medium modifies heavy ion abundances and energy spectra in large gradual SEP events. In some large events, self-generated waves can enhance particle trapping and increase the acceleration efficiencies of the CME shocks

Moreover, the presence of very high $M_{\mathrm{fm}}$ values along open field lines crossing the shock in the vicinity of the neutral line, corresponding to the heliospheric plasma sheet, suggests that the neutral line could be a favorable region for particle acceleration, although spatially limited. Thus, it is necessary a good connectivity between the shock regions crossing the vicinity of the tip of streamers and the associated neutral line, e.g., through a large-scale magnetic flux rope or any complex magnetic field structure.

By using the same approach, Plotnikov et al. (2017) reconstructed the evolving shock front and its properties for three far-side CME events (2013 Oct. 11, 2014, Jan. 06 and Sep. 01) which were associated with LPGREs, as observed by the Fermi/LAT (Large Area Telescope) at energy > $100 \mathrm{MeV}-300 \mathrm{GeV}$, and SEP events measured in situ at $1 \mathrm{AU}$ (by SOHO and STEREO A, B). They obtained that for all the three events, the shock became super-magnetosonic $\left(M_{\mathrm{fm}}>1\right)$ and magnetically connected to the visible solar surface within 10-15 min of the start of the flare and just before the onset of the $>100 \mathrm{MeV}$-ray emission, showing a quasi-perpendicular geometry at the flanks during the bulk of the gamma-ray emission. Moreover, by comparing the SEP electron and gamma-ray onset times and the computed electron to proton ratios at the Sun and in space (within a factor 5), they concluded that the same shock processes are responsible for both LPGREs and the production of SEPs. Nevertheless, they found no clear correlation between the shock Mach number levels and the intensity of the gamma-ray 
flux measured by LAT, suggesting that a more complicated physics might be at work, which requires further investigation.

Furthermore, Kouloumvakos et al. (2019) derived the shock parameters from 3D modeling of the coronal pressure waves and compared them with properties of SEP events over an extended dataset. They analyzed a number of 33 SEP events with energy > $50 \mathrm{MeV}$ clearly observed at least at two interplanetary locations by $\mathrm{SOHO}$ and STEREO, and computed the correlation between the peak intensity during the prompt phase and several shock parameters at the shock regions magnetically well-connected with the observers. Correlations with shock speed, compression ratios, and Mach numbers were found to be significant for well-connected field lines, having Pearson's correlation coefficients of $65 \%$, $56 \%$, and $72 \%$, respectively, supporting previous results (Rouillard et al. 2016; Plotnikov et al. 2017; Afanasiev et al. 2018). On the other hand, no significant correlation was found between the SEP peak intensity and the shock angle as well as no energy dependence on any performed correlation. Finally, shock waves were found to become super-critical at a median distance of 3 RS, while solar particles to be released $\sim 15-20 \mathrm{~min}$ after the shock waves become super-critical and have connected magnetically with the SEP observing point.

Kozarev et al. (2015) analyzed the initial phase of a CME on 2011 May 11 by combining observations from the SDO/AIA images in the time interval 02:12-02:31 UT and the following models: 1) the Coronal Shock Geometric Surface (CSGS) model which produces a three-dimensional spherical dome surface, propagating through the corona with speed and radius based on the observed time-dependent position of the EUV coronal bright front (describing the shock front) in the radial direction; 2) the Potential Field Source Surface (PFSS) magnetic field model, providing a global coronal vector magnetic field solution for a $3 \mathrm{D}$ grid of polar coordinates. They estimated the time-dependent orientation of the wave/shock with respect to the coronal magnetic fields between 1.1 and $1.5 \mathrm{Rs}$, the part of the coronal surface which shock-accelerated particles during the initial stages as well as heliospheric connectivity during the shock passage in the low corona. They found that the fieldshock angle changes significantly throughout the evolution of the shock surface, with higher values preferentially near the flanks, although they reached almost $90^{\circ}$ near the shock nose as well in the last phase of the event. Moreover, the open field lines crossed by the shock in the second half of the event were likely both related to high angle values and magnetically connected to the Earth. Thus, the authors concluded that the shock acceleration efficiency and particle release is considerably higher in the second part of the shock evolution in the AIA FOV.
Recent MHD simulations of the CME expansion have shown the formation of shocks or strong compression regions at low coronal heights $(<2$ solar radii). As a result of the CME's rapid acceleration, shocks and strong compressions appear on the flank of the CME, showing a large negative velocity divergence and creating the conditions that lead to rapid particle acceleration. By analytically solving the Parker equation in the presence of size-limited acceleration regions as obtained by MAS (Magnetohydrodynamic Algorithm outside a Sphere) simulations, Schwadron et al. 2015) showed that broken power laws can be naturally obtained due to pronounced effects of particle diffusion and particle escape. As a matter of fact, the finite size of the shock or compression limits the maximum energy gain of the particles, because the magnetic field line (or flux bundle), near which these particles are accelerated, moves off of the accelerator, i.e., the shock or compression, leading to the formation of a broken power-law in the particle distribution. Moreover, the break energy and the spectral index of the second power law increase with the shock angle, the size of the shock, the CME driver speed, and with reductions in the rigidity dependence of the scattering mean free path.

The effect of large-scale streamer-like magnetic configuration on particle acceleration at coronal shocks has been investigated by considering a CME-driven shock propagating through a streamer-like magnetic field (Kong et al. 2017) or from its flank (Kong et al. 2019), i.e., when the streamer is rotated with respect to the CME propagation direction. By numerically solving the Parker transport equation with both parallel and perpendicular diffusion in such configuration, Kong et al. (2019) found that the primary sources for particle acceleration are located at different regions and vary significantly as the shock propagates and expands, depending on the particle energy and on time. In particular, the acceleration of particles to more than $100 \mathrm{MeV}$ mainly occurs in the shock-streamer interaction region close to the shock flank, where the shock is quasi-perpendicular and closed magnetic fields are present, favoring particle trapping upstream of the shock, both conditions increasing the acceleration efficiency. They also obtained broken power laws for the particle spectra up to 100 $\mathrm{MeV}$ integrated over the simulation domain. Thus, the streamer-like magnetic field can play a critical role in producing gradual solar energetic particle events and they may be a mixture of two distinct populations accelerated in the streamer and open field regions, having different acceleration rates. Nevertheless, these results might be affected by the two-dimensional treatment, leading to non-zero divergence of the magnetic field at the shock front and possibly to mismatch parallel diffusion and perpendicular diffusion. 
Some previous models attribute the generation of double power-laws and Q/M-dependent spectral breaks to the trapping and subsequent rigidity-dependent escape of ions from the shock during the SEP acceleration processes via DSA at CME-driven shocks in the solar corona (Li et al. 2009a; Tylka and Lee 2006). In the Schwadron et al. (2015) lower coronal SEP acceleration model, the finite size of the ICME shock and stronger $\mathrm{Q} / \mathrm{M}$-dependence of the diffusion coefficient facilitates particle escape from the acceleration region, which reduces the $\boldsymbol{E}_{\boldsymbol{o}}$ and steepens or softens the higher energy spectral slope. Conversely, in the Schwadron et al. (2015) model, the weaker Q/M dependence inhibits particle escape, which increases the break energy and flattens the higher energy spectral slope. Indeed, Desai et al. (2016b) were able to infer key properties such as the strength, obliquity, and turbulence conditions associated with the corresponding near-Sun CME shocks using the spectral properties of those 1 AU SEP events where the observed Q/M-dependent spectral breaks were interpreted to be consistent with DSA shock acceleration rather than transport-dominated processes. Recently, Tan et al. (2017) also found that the high-energy $\mathrm{Ne} / \mathrm{O}$ ratio is well correlated with the source plasma temperature of SEPs, and used the variability of $\mathrm{Ne} / \mathrm{O}$ and $\mathrm{Fe} / \mathrm{O}$ ratios to investigate the accelerating shock properties.

In contrast, Li and Lee (2015), Zhao et al. (2016a, b), and Zhao et al. (2016b) argue that, in some large SEP events, single power-law spectra injected by near-Sun ICME shocks can exhibit spectral breaks at $1 \mathrm{AU}$ due to $\mathrm{Q} / \mathrm{M}$-dependent scatter-dominated transport in the IP medium. Specifically, Li and Lee (2015) showed that particle scattering and diffusion from the Sun en route to 1 AU could alter a single proton power law into three distinct power laws by the time the shock arrives at 1 $\mathrm{AU}$, suggesting that scatter-induced particle propagation in the IP medium can also result in spectral breaks at 1 AU even if the ICME shock-accelerated spectra are pure power laws; in this case, the $\mathrm{Q} / \mathrm{M}$ dependence ( $\alpha$ parameter) has an upper limit of $\sim 1.4$. Thus, in cases where the spectral breaks are interpreted to be caused primarily by interplanetary transport effects, the observed temporal evolution of the heavy ion abundances and spectral breaks can provide insights into the interplanetary turbulence conditions encountered by the SEPs during their transit to $1 \mathrm{AU}$ ( $\mathrm{Li}$ and Lee 2015; Zhao et al. 2016a; Zhao et al. 2016b). On the other hand, another functional form was also found to reproduce the proton energy spectrum during SEP events (Laurenza et al. 2013, 2015), being the Weibull distribution the best fit for the event-integrated spectrum and separately, during the prompt and energetic storm particle phases. A theoretical derivation of the Weibull spectrum was provided (Laurenza et al. 2016; Pallocchia et al. 2017) in the framework of the acceleration by "killed" stochastic processes exhibiting power-law growth in time of the velocity expectation, such as the classical Fermi process, or alternatively, by the shock-surfing acceleration. Thus, those authors suggested that a scenario in which different mechanisms could account for particle acceleration at shocks in different energy ranges, the stochastic or shock surfing acceleration contributing significantly to the acceleration of high energetic particles, in addition to the DSA at lower energies.

\subsubsection{SEP transport}

The well-separated SEP observations with the STEREO mission have also lent themselves to study the transport of SEPs in more detail. The comparison of multispacecraft observations with results of $2 \mathrm{D}$ or $3 \mathrm{D}$ models solving the focused transport equation allowed to study not only transport along the mean magnetic field but also perpendicular to it. It was found that the role of efficient transport perpendicular to the mean magnetic field can eventually be much stronger than expected (Dresing et al. 2012) and might play an important role, among extended injection and acceleration regions, in the longitudinal spreading of SEPs (Dröge et al. 2014; Dröge et al. 2016) but also in creating asymmetries in their longitudinal distribution at $1 \mathrm{AU}$ ( $\mathrm{He}$ and Wan 2015; Strauss et al. 2017).

The main theories attempting to describe perpendicular transport are diffusion, i.e., by particle scattering at magnetic field irregularities resonant with the particles' gyro radius (Zhang et al. 2009; Giacalone and Jokipii 2012; Dröge et al. 2010; Dröge et al. 2014; Dröge et al. 2016) and field line meandering. Laitinen et al. (2016) and references therein propose a combined scenario with particles remaining on turbulently meandering field lines early in the event which turns over into diffusive transport at a later phase, both leading to particles spreading perpendicular to the mean field. While a solely diffusive approximation is not able to explain efficient perpendicular transport at the same time like the presence of sharp SEP drop out events (Mazur et al. 2000), the model of field line meandering is. Furthermore, it is able to explain too early SEP onsets caused by a propagation path length shorter than the nominal Parker spiral length (Laitinen and Dalla 2019). However, both diffusion and field line meandering approaches are struggling to explain the extreme widespread events observed with the STEREO and close-to-Earth spacecraft. This suggests that such events are not only caused by efficient perpendicular transport but by a combination of transport and an extended injection and/or acceleration region. The limited number of well-separated observers during the STEREO era was not fully sufficient to constrain the injection size and the transport 
conditions at the same time which is expected to improve significantly during the next solar cycle with the presence of new missions like Parker Solar Probe and Solar Orbiter.

An important step taken in SEP transport modeling is the inclusion of a realistic solar wind background. Wijsen et al. (2019a) use the data-driven EUropean Heliospheric FORecasting Information Asset (EUHFORIA, Pomoell and Poedts 2018) to generate a background solar wind for their SEP transport code. This allows, on the one hand, the study of the effect of solar wind streams on the SEP propagation and also on adiabatic energy changes (Wijsen et al. 2019b). On the other hand, case studies with a realistic background field will be possible accounting for the effects of transient structures leading to non-Parker field configurations (Leske et al. 2012)

Pacheco et al. (2019) have re-visited 15 relativistic electron events observed with the Helios spacecraft in the 1970s and 1980s using the 1D transport code by Agueda et al. (2008), which allows to infer the solar release time profiles and the values of the radial mean free path. Short injection duration $(<30 \mathrm{~min})$ events were only found in $30 \%$ of the cases compared to longduration events ( $>30 \mathrm{~min}$ ) otherwise. The radial mean free paths, observed at spacecraft locations between 0.31 and $0.94 \mathrm{AU}$, vary between 0.02 and $0.27 \mathrm{AU}$. Agueda and Lario (2016) found indications that the strength of the interplanetary scattering varies with the size of the solar parent event suggesting that the energetic particle population itself generates waves. However, Pacheco et al. (2019) did not find such a dependency in their sample. While self-generated waves are an accepted phenomenon for ions, which plays also an important role in proton acceleration at shocks (Bell 1978) (see also section 6.3.1), their presence in relation to solar energetic electrons remains elusive. The four consecutive Helios events studied by Agueda and Lario et al. (2016) are also a famous example used to illustrate the radial effect of SEP transport: the same SEP events were observed by the close-to-Earth spacecraft IMP-8, but the four distinct impulsive increases seemed to have merged into only one gradual event. However, as discussed and modeled by Agueda and Lario et al. (2016) and Strauss et al. (2017), not only radial scattering alone but most likely the contribution of perpendicular diffusion is responsible for the loss of the detailed SEP event structure at $1 \mathrm{AU}$.

\subsection{Conclusion}

In conclusion, for a full understanding of the SEP acceleration, injection, and transport processes which altogether determine the variable particle observations discussed so far, additional systematic, multi-spacecraft studies are needed. SEP observations provided by the
Parker Solar Probe (McComas et al. 2016) and Solar Orbiter (Rodríguez-Pacheco et al. 2020) missions in the inner heliosphere in conjunction with modeling efforts will be the basis of upcoming advancements in our understanding.

\section{Stream interaction regions/co-rotating interaction regions}

\subsection{Introduction}

The solar corona is structured by open and closed magnetic field regions that transition at a certain distance as open field into interplanetary space (see Fig. 29). The solar atmosphere is permanently in a state of dynamic energy release and renewal, e.g., structures constantly interact with each other. Flares and CMEs are mostly related to active regions and filament eruptions. Other solar features that can cause strong geomagnetic events are stream interaction regions (SIRs) which are related to coronal holes, known as areas on the Sun with predominantly open magnetic field. In that respect, the knowledge about the $360^{\circ}$ structuring of interplanetary space is a key input parameter for Space Weather forecasting. As CMEs are affected by the MHD drag which is in quadratic dependence on the ambient solar wind speed, fast streams may strongly influence the predictions of arrival times and impact speeds of CMEs. The structured interplanetary space also actively affects SEP propagation and causes by itself geomagnetic storms. During the solar minimum phase, the energy input into Earth's magnetosphere by CIRs is similar to that of CMEs (Richardson et al. 2001; Tsurutani et al. 2006).

SIRs are related to the high-speed streams originating from regions of open magnetic fields along which plasma is accelerated and may easily escape from the Sun. Primarily open magnetic fields (and flux) are observed within coronal holes, dark regions of lower temperature and density as observed in EUV and SXR. Harvey and Recely (2002) describe the evolution of CHs based on observations during solar cycles 22 and 23. Isolated high-latitude $\mathrm{CHs}$ evolve into polar $\mathrm{CHs}$, where they appear as stable and rigidly rotating objects, which may exist for several years. Approaching the descending phase of a solar activity cycle, polar $\mathrm{CHs}$ extends to lower latitudes that consequently change the solar wind in the ecliptic (i.e., encompassing the planets) quite dramatically. This evolutionary process is revealed in EUV data from the clear change of morphology (area, shape) and location of CHs. Wang and Sheeley Jr 1990 describe the magnetic structure of $\mathrm{CHs}$ and find that there is a close relation between the flux tube expansion and the underlying photospheric magnetic field. Recent results show that the evolution of the $\mathrm{CH}$ area is not correlated to the evolution of the underlying magnetic field, indicating that the magnetic field inside a $\mathrm{CH}$ does not drive 


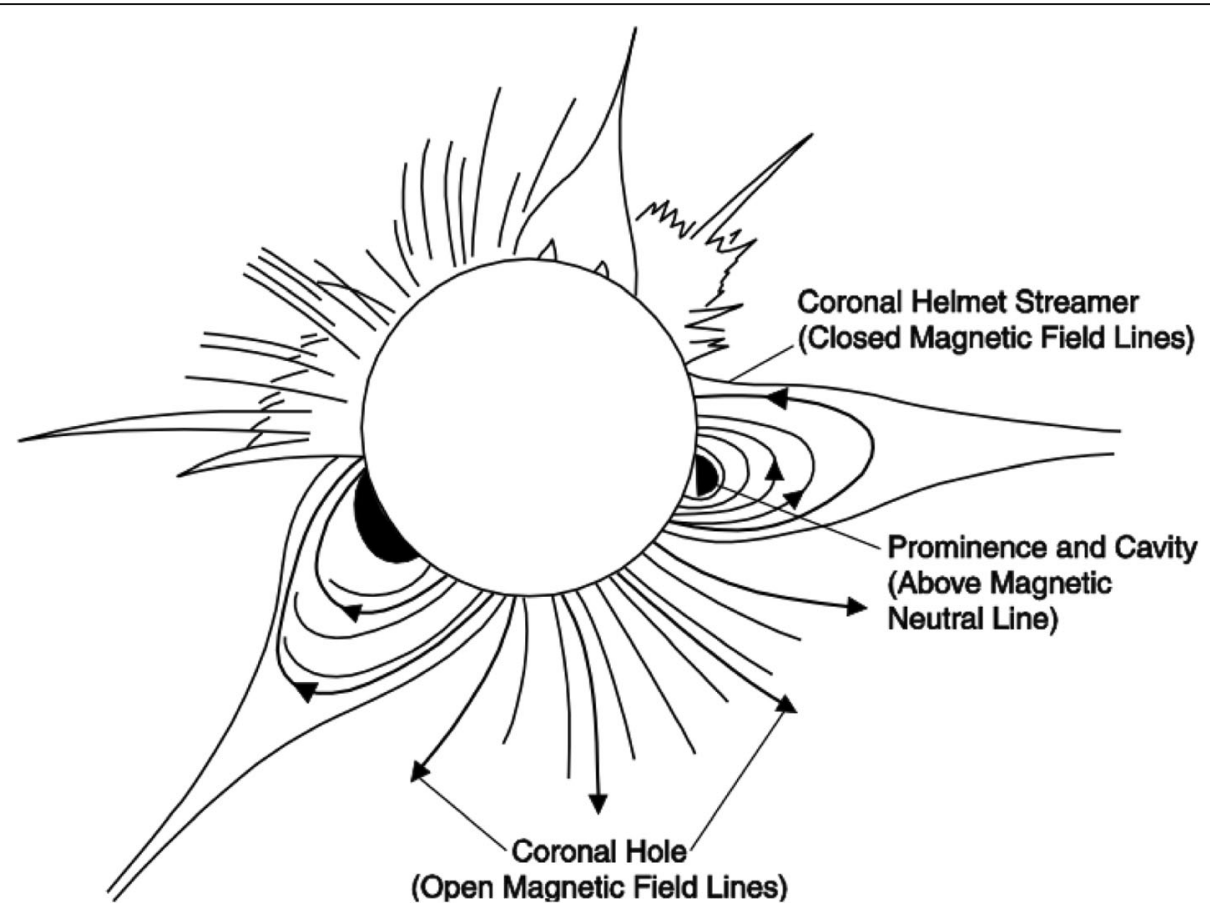

Fig. 29 Sketch of the open-closed magnetic field structures on the Sun. Adapted from Fig. 4.8 of the book "Introduction to Space Physics" edited by Kivelson and Russell et al. (1995)

its evolution (Heinemann et al. 2020). SIRs have been observed throughout the heliosphere by a large variety of spacecraft, such as Helios in the inner heliosphere, by ACE, Wind, DSCOVR near Earth at $1 \mathrm{au}$, by Voyager and Pioneer in the outer heliosphere, and also out of the ecliptic by Ulysses.

Due to the quasi-stationary location of low-latitude $\mathrm{CHs}$, the interaction of high- and slow-speed solar wind streams results in compression of plasma and magnetic fields. As the plasma is of enhanced speed compared to neighboring regions, interactions between fast and slow solar wind streams occur at certain distances from the Sun (cf. Fig. 30). Here the leading edge of the interaction region forms a forward pressure wave that propagates into the slower plasma ahead. Likewise, the trailing edge is a reverse pressure wave (see review by Gosling and Pizzo 1999). Since $\mathrm{CHs}$ are slowly evolving but longlived structures, these interaction regions recur with every solar rotation and are then called co-rotating interaction regions (CIRs). On the large scale, it is assumed that their interplanetary dynamics can be described by ideal MHD equations.

One of the characteristics of SIRs, measured at 1AU distance, is the so-called stream interface, characterized by an abrupt drop in density, simultaneous rise in proton temperature and gradual increase in speed, and an eastwest flow deflection, i.e., the region separating the originally slow, dense plasma from originally fast thin plasma back at the Sun (e.g., Wimmer-Schweingruber et al. 1997). Furthermore, the stream interface is usually preceded by a density increase due to the compression (compression region) and is often associated with a sudden change in the magnetic polarity (sector boundary), and a gradually rising solar wind speed profile (Jian et al. 2009). The change in the magnetic polarity is related to the alternation of magnetic sectors, referring to the neutral line (heliospheric current sheet). The number of magnetic sectors changes with the solar cycle, typically there are 4 sectors, but it can get more complex during solar maximum. Shocks on both sides of the interaction region develop more strongly further out in the heliosphere at 2-3 AU (forward and reverse shock).

Because SIRs/CIRs rotate in the direction of planetary motion, the high-speed solar wind streams emanating from a centrally located coronal hole arrives at Earth about 4 days later (see Fig. 31). Typical HSS intervals following CIRs, may drive prolonged geomagnetic activity and cause strong high energy particle enhancements in the Earth's radiation belts (Reeves et al. 2003; Miyoshi et al. 2013; Kilpua et al. 2015). In detail, the strength and impact of geomagnetic storms depends, beside the impact speed, most importantly on the north-south component, $B_{\mathrm{z}}$, of the magnetic field of the solar wind stream (see also Krauss et al. 2015). In the case of CIRs, the cause of the disturbances is often Alfvenic waves in the HSS (Tsurutani et al. 2018). 


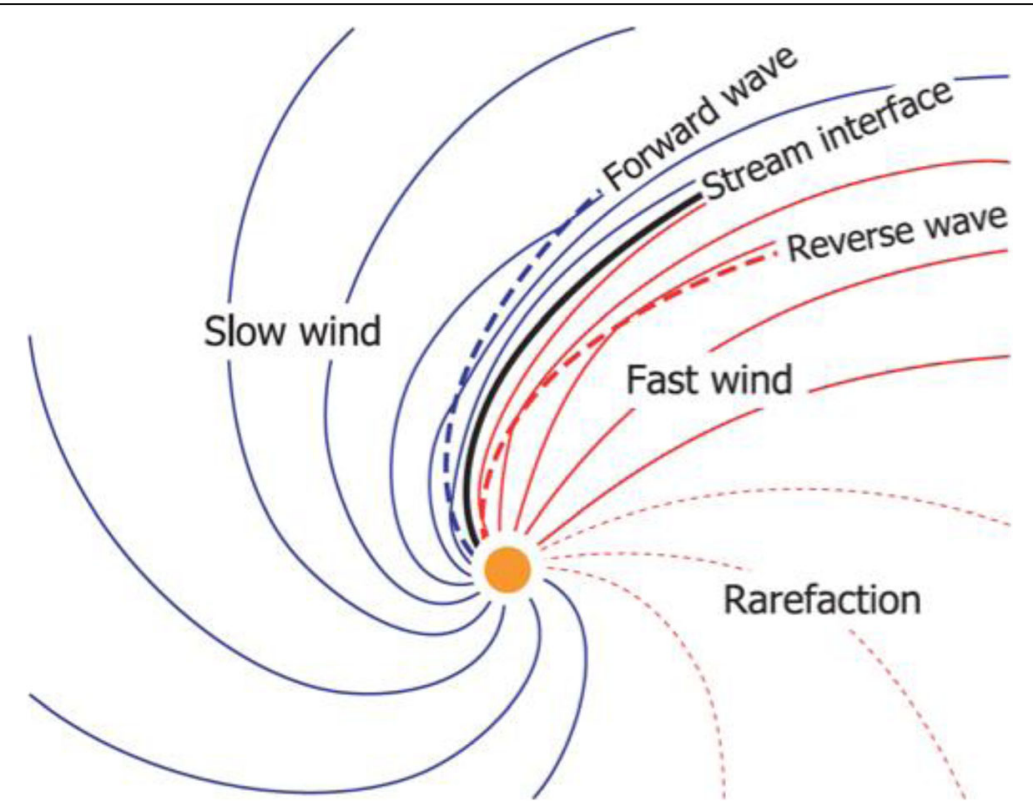

Fig. 30 Sketch of an SIR as seen in the ecliptic plane showing the fast (slow) solar wind in red (blue). The frozen-in magnetic field lines become aligned with the stream interface by the reverse (forward) wave. Taken from Owens and Forsyth (2013) adapted from Gosling et al. (1981)

\subsection{Progress in observations}

The evolution of long-lived coronal holes, closely related to SIRs/CIRs, can be studied in detail using multiple views on the Sun from combined STEREO and Earth imagery. Using spacecraft separated by $120^{\circ}$, studies of changes in the large-scale EUV structures over time and their relation to the in situ measured solar wind can be performed. It was found that coronal holes undergo evolutionary patterns revealing a growing and declining phase where area increases and decreases again over several solar rotations (Heinemann et al. 2018a; Heinemann et al. 2018b; Heinemann et al. 2020). The slow evolution of coronal hole areas and the steady-state of the related solar wind streams are found to be well related to the solar wind speed measured in situ at 1 AU (Nolte et al. 1976; Veronig 2007a; Abramenko et al. 2009; Karachik and Pevtsov 2011; Rotter et al. 2012; Tokumaru et al. 2017). With this well-known area-speed relation, empirical forecasting tools for the "pure" background solar wind on the basis of coronal hole area measurements are performed on a regular basis as depicted in Fig. 32 (Vršnak et al. 2007a; Rotter et al. 2012; Reiss et al. 2016). By understanding the photospheric and coronal evolutionary characteristics of $\mathrm{CH}$, one can aim to gain a better understanding and in turn improve the forecast of CIRs (see Heinemann et al. 2018a, b). Temmer et al. (2017) showed in a case study that the evolutionary trend visible in the $\mathrm{CH}$ area is matched by the trend of the peak velocity of the associated HSS over the lifetime of the $\mathrm{CH}$. This is an important indicator that the highspeed peak velocity to $\mathrm{CH}$ area relation also persists over a CH's evolution. They also found that the total perpendicular pressure at the stream interface and the in situ magnetic field at the $\mathrm{B}$ - peak and the $\mathrm{v}$ - peak do not show the same evolutionary profile. The forecasts are found to be most successful for periods of low solar activity, as during increased solar activity transient events, such as CMEs, strongly disturb the rather stable solar wind outflow for several days (up to 5 days; see Temmer et al. 2017); cf. also Janvier et al. (2019).

As the high-speed solar wind streams emanate from $\mathrm{CHs}$ which are low dynamic structures, CIRs can be forecasted with long lead times. Based on that a variety of persistence models were developed. Under the assumption of persistence, in situ measurements of the solar wind plasma flow from L1 and varying STEREO vantage points provide a forecast for Earth position with lead times of up to 27 days (depending on the exact STEREO spacecraft position). The closer the measuring spacecraft is ahead or behind Earth, the less the effect of the temporal evolution of the solar wind profile (Opitz et al. 2009). Implementing the actual changes of $\mathrm{CH}$ areas (from EUV data) into such simple forecasting tools can improve the forecast quality (Temmer et al. 2018). Lead times with about 4.5 days could be achieved when using data from an instrument permanently located at the Lagrangian point L5 $\left(60^{\circ}\right.$ behind Earth; ESA preparation for the future L5 mission "Lagrange"). However, it is pointed out that the latitudinal offset between the measuring spacecraft is limiting the accuracy of persistence modeling (Owens et al. 2019) as the streams flow speed profile is rather depending on the 


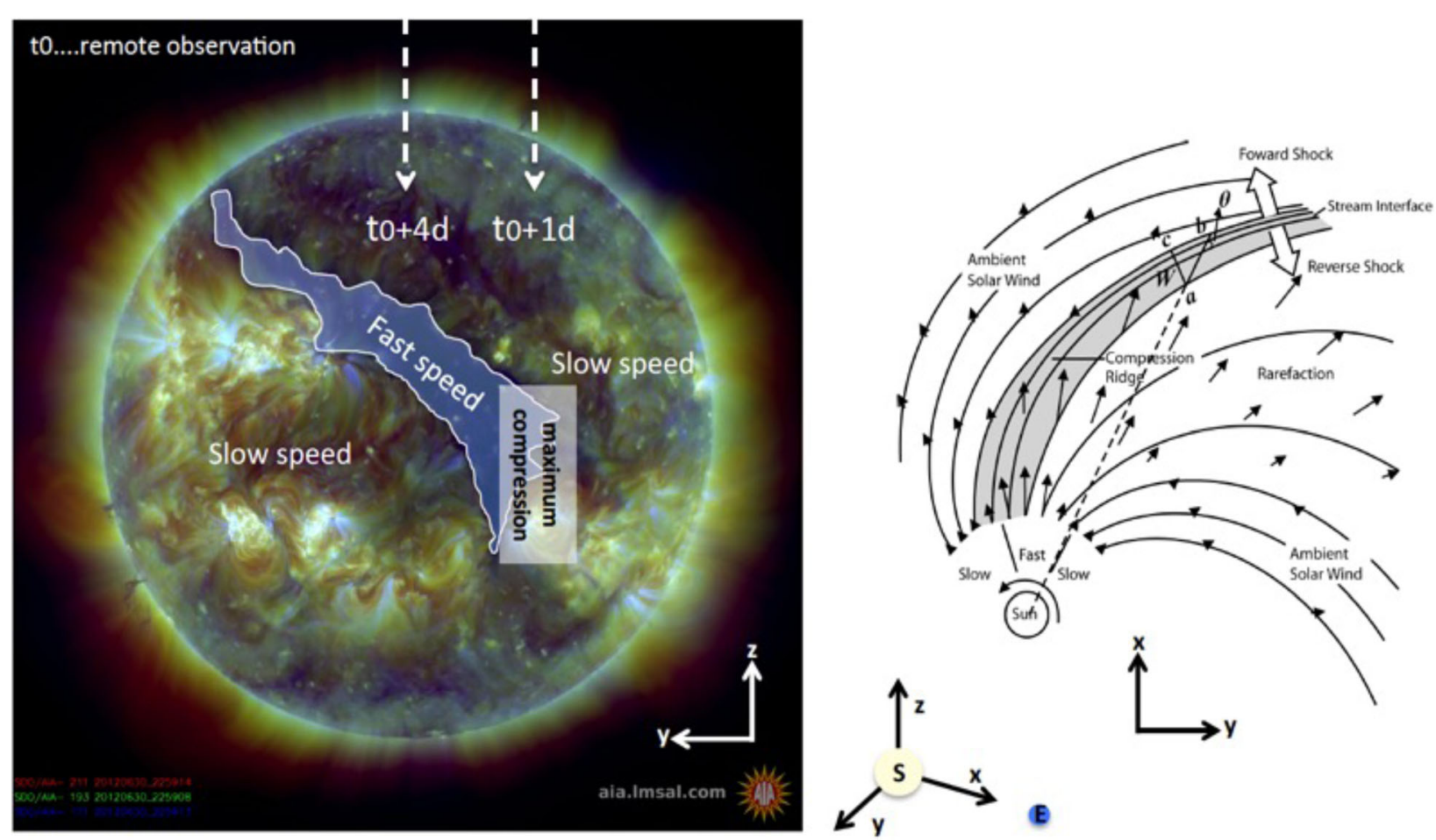

Fig. 31 Left: SDO/AIA composite image of the wavelength channels 211-193-171A from June 30, 2012, showing the reduced density region of a coronal hole (shaded area). At the time $t_{0}$, the central position of the coronal hole is extracted from remote data. At about $t_{0}+1$ day, the maximum in the density/magnetic field and at about t0 $+4 \mathrm{~d}$ the maximum in the speed/temperature is observed from in situ data at $1 \mathrm{AU}$. Right: Cartoon by Pizzo (1978) to illustrate the fundamental processes involved in the 3D dynamics of stream evolution

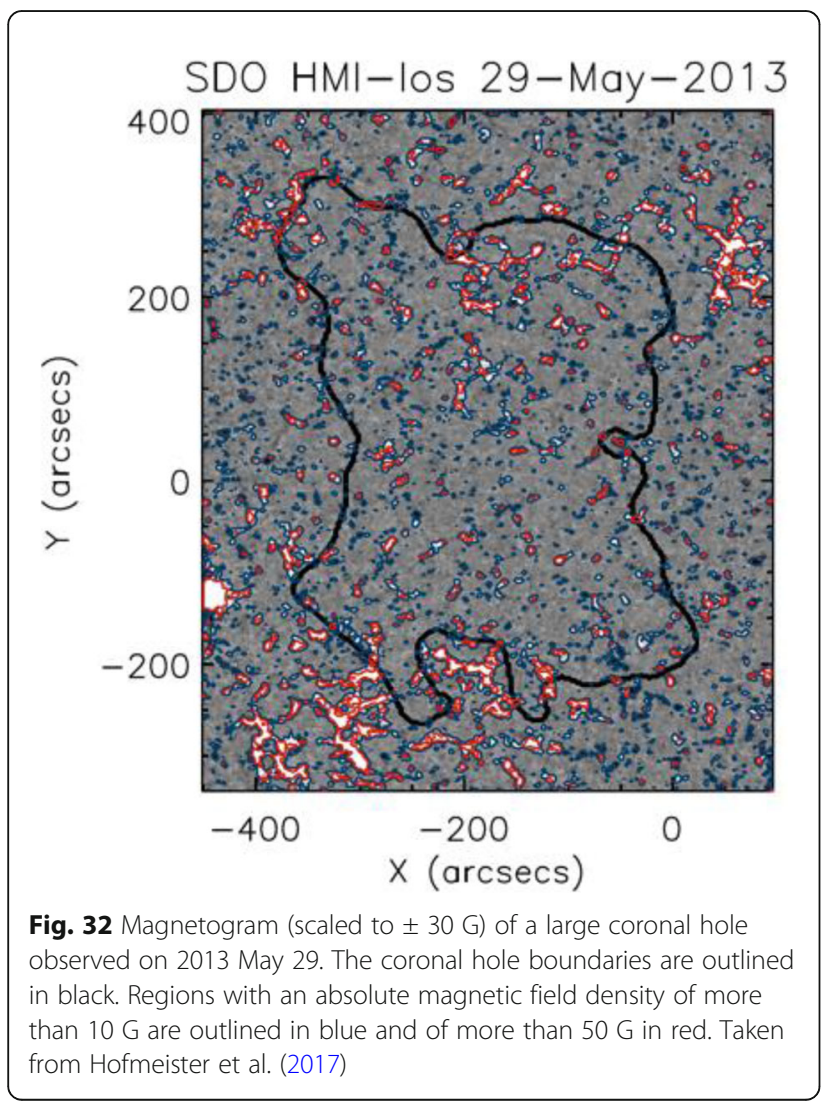

latitudinal range of the center of mass (Hofmeister et al. 2018). Recent studies also found latitudinal differences in the geoeffectiveness of events which are caused by variations in the interplanetary magnetic field due to closed flux ropes (CME magnetic structure) and compression regions - CME shock-sheath or SIR (Huttunen et al. 2008; Yermolaev et al. 2017). Together with the warping of the heliospheric current sheet, the forecasting of high-speed solar wind structures is rather complex, but considering the uncertainties, nevertheless, a good estimation of the flow approaching Earth can be given.

Forecasting solar wind structures in interplanetary space serves also as important information for analytical CME propagation models and Space Weather models. However, SIR/CIRs may not only influence the propagation of CMEs but also the evolution of its internal magnetic structure, as the compression region may represent an obstacle which can hamper the CME expansion (see e.g., Dumbović et al. 2019). Therefore, under the Space Situational Awareness Program of the European Space Agency forecasting services using empirical, and numerical models for the solar wind are available (see http:// swe.ssa.esa.int). In that respect, a new four-plasma categorization scheme for the solar wind is given by $\mathrm{Xu}$ 
and Borovsky (2015) that can be used for the automatized detection scheme for solar wind and CMEs (private communication with S. Vennerstroem, 2015). As the forecasting/nowcasting quality is still not sufficient for producing reliable Space Weather alerts, we need to better understand and closely monitor SIRs/CIRs. Main aims for the near future are to (1) verify and evaluate background solar wind models and with that improve the input for CME propagation models (as discussed in Section 4.1 and 4.2) and more accurately predict periodic and recurrent geomagnetic effects from CIRs. In general, single events are easier to forecast compared to multiple events featuring CME-CME interactions, or interactions of CMEs with CIRs. In order to better understand the fast flow plasma in interplanetary space, research on their sources, $\mathrm{CHs}$, is of utmost importance (Wilcox and Howard 1968). By better understanding the physics behind $\mathrm{CH}$ evolution we may improve their forecasting capability. With that, we will also gain more insight into the ejection and acceleration processes that define high-speed streams. This is of timeliness as we can exploit data from the NASA mission Parker Solar Probe (PSP) (Fox et al. 2016) measuring the near-Sun space and with that regions where the solar wind actually gets accelerated.

Hofmeister et al. (2017) in a statistical study of 288 low-latitude coronal holes during the time range of January 01, 2011-December 31, 2013, and Hofmeister et al. (2019) in a statistical study of 98 coronal holes shed light on the magnetic fine structure in photosphere underlying the projected $\mathrm{CH}$ boundary. Using SDO/ HMI line-of-sight magnetograms, they showed that the magnetic field is made up of a very weak slightly asymmetrically skewed background field ( $\left|\mathrm{B}_{\mathrm{BG}}\right|=0.2-1.2 \mathrm{G}$ ) and small unipolar magnetic elements. These small unipolar magnetic elements contain most of the signed magnetic flux that arises from coronal holes. It was found that the area that these unipolar, usually longliving (lifetimes $>40 \mathrm{~h}$ ), magnetic elements cover determines the total signed flux of a coronal hole (see Fig. 32). These magnetic elements are important in the context of solar wind acceleration, propagation and forecasting as they are suspected to be the footpoints of flux tubes or magnetic funnels. Flux tubes, or clusters of magnetic fibers open towards interplanetary space, have been found to be the small-scale source regions of the plasma outflows within CHs (Hassler et al. 1999; Tu et al. 2005). Wiegelmann et al. (2014) showed, using SUMER data and magnetic field extrapolations, that the regions showing high outflow velocities correspond to strong unipolar flux concentrations within $\mathrm{CHs}$ (see Figure 36 in Section 7.3 ("Progress in theory and simulations")). The substructure of these flux tubes, e.g., a bundle of magnetic fibers, is represented by the abundance of magnetic bright points (MBPs) within magnetic elements as observed in high-resolution HINODE/SOT G-band filtergrams (Hofmeister et al. 2019). This shows the highly structured magnetic field configuration of $\mathrm{CHs}$ in comparison to the previously often assumed rather uniform configuration. This structuring also carries out into the solar wind structure in interplanetary space as shown by the first PSP observations of HSS that were found to show a strongly structured and perturbed speed profile (Bale et al. 2019).

Though harder to evaluate and forecast, interaction events between SIR/CIR and CMEs are of special interest. Interaction events can lead to significant increase in geomagnetic effects when compared to individual events of similar strength ( $\mathrm{He}$ et al. 2018; Dumbović et al. 2019). Heinemann et al. (2019a) showed in a case study of a CME interacting and propagating within a HSS on June 22, 2011, that the dynamic pressure of the SIR/CIR followed by the CME shock signature within the HSS induces wave-like flaring-motion into the Earth's magnetopause and causes, due to enhanced magnetopause currents, a much stronger Sym-H value than would have been expected. Due to the rather small and weak CME the effects were still only moderate. Also, enhanced substorm activity was recorded.

In a recent paper by Jian et al. (2019), physical properties of a large sample of slow-to-fast SIRs were investigated using STEREO-A and -B data. They identified 518 pristine SIRs, of which more than $50 \%$ are associated with crossings of the heliospheric current sheet (HCS) and are of slow speeds but higher densities, and of increased dynamic and total pressure compared to those without HCS (see Fig. 33). In that respect, HCS related SIRs can be classified as more effective in terms of Space Weather.

The detection and extraction of reliable $\mathrm{CH}$ areas from operational solar observations is extremely important, not only for solar wind forecasting using the area-speed relation, but also for investigating the magnetic open flux on the Sun. At the present, most extraction methods focus on EUV observation taken by SDO/AIA, SOHO/EIT, GOES/SUVI and/or STEREO/EUVI. Due to the optimal filter sensitivity and high contrast, wavelengths of highly ionized iron (e.g., Fe XII: 193/195 Å) are often used. Methodologically, intensity-based methods are the go-to choice, with some form of intensity thresholding being the preferred choice. Intensity threshold methods include the CHARM algorithm (L. Krista PhD Thesis, Krista 2012), which uses local intensity histograms to determine a fitting threshold; a fixed threshold based on the median solar disk intensity (Rotter et al. 2012, 2015; Reiss et al. 2015; Boucheron et al. 2016; Hofmeister et al. 2017; Heinemann et al. 2018a); a dual-threshold growing algorithm (Caplan et al. 2016) and a supervised intensity threshold approach modulated by the intensity gradient 

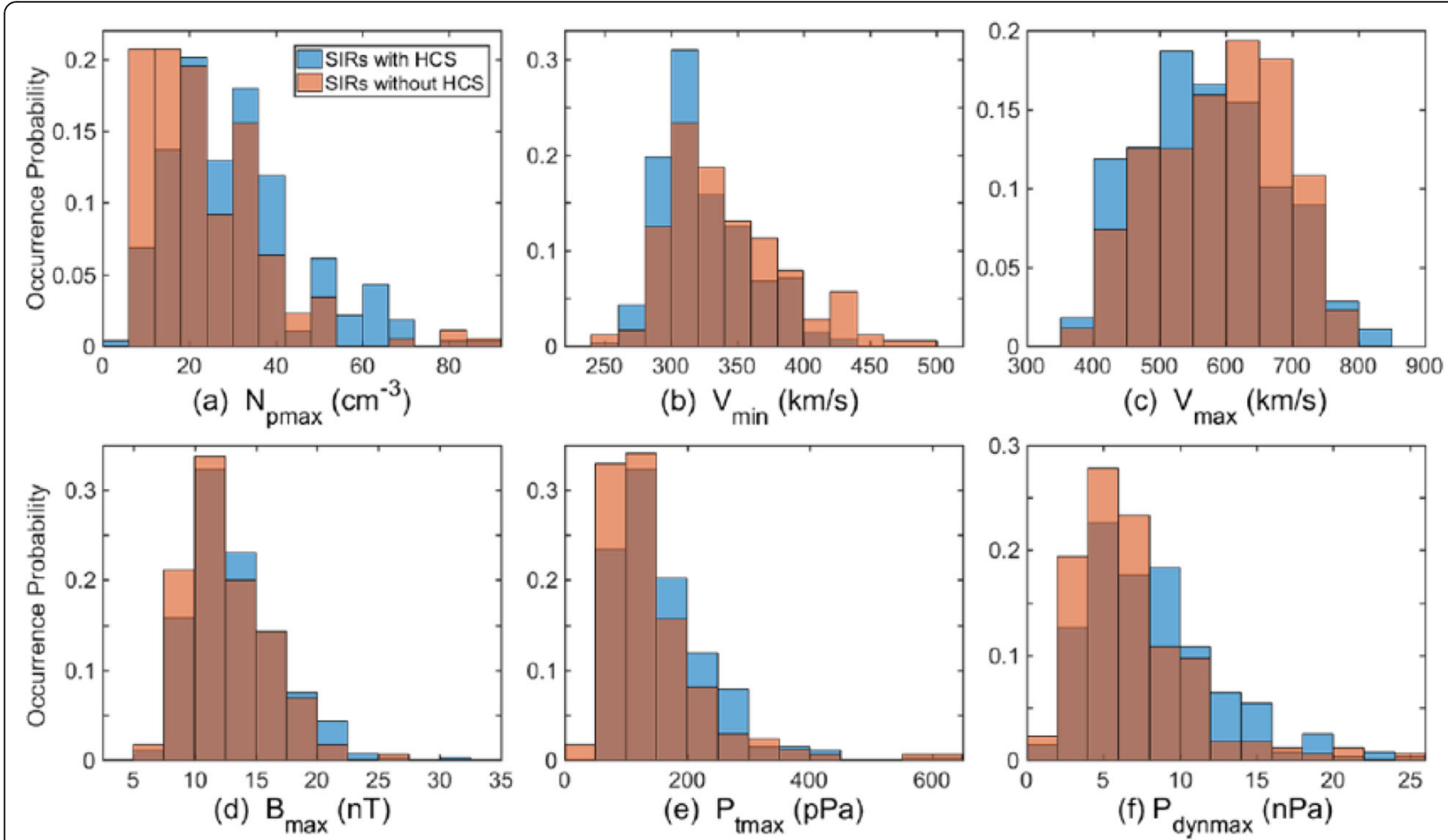

Fig. 33 The comparison of occurrence probabilities between SIRs with an HCS crossing (blue bars) and without any HCS crossing (orange bars) for the following parameters: a maximum $N_{\mathrm{p}}$, b minimum speed, $\mathbf{c}$ maximum speed, $\mathbf{d}$ maximum $B$, e peak $P_{\mathrm{t}}$, and $\mathbf{f}$ peak dynamic pressure. The brown shaded regions are the overlapping regions between SIRs with an HCS and the SIRs without any HCS crossing. Taken from Jian et al. (2019)

perpendicular to the $\mathrm{CH}$ boundary $(\mathrm{CATCH}$; Heinemann et al. 2019a, b). A multi-wavelength approach was developed by Garton et al. (2018) in the form of the multi-thermal emission recognition algorithm CHIMERA. A spatial possibilistic clustering approach was taken by Verbeeck et al. (2014) which is available as the SPOCA algorithm. Recently, with the dawn of machine learning, new methods, utilizing the increased computational performance have also emerged to provide an additional tool to identify and extract coronal holes (e.g., Illarionov and Tlatov 2018).

Using various techniques, several $\mathrm{CH}$ datasets were gathered (especially for $\mathrm{CH}$ areas) that are freely available. Automatically created SPOCA boundaries of $\mathrm{CHs}$ are available via the Heliophysics Events Knowledgebase (HEK: https://www.lmsal.com/hek/index.html), the automated coronal hole detection and extraction using three SDO/AIA wavelengths $(171,193,211 \mathrm{~A})$ CHIMERA is available via SolarMonitor (https://www.solarmonitor. org/) and an extensive, manually checked, $\mathrm{CH}$ catalog covering the SDO-era (2010-2019) created using CATC $\mathrm{H}$ is available via the VizieR catalog service (http:// cdsarc.u-strasbg.fr/viz-bin/cat/J/other/SoPh/294.144).

A recent chain of studies used data from HINODE, SOHO, and TRACE to study small-scale changes in the
$\mathrm{CH}$ boundary. In a first step, Madjarska and Wiegelmann (2009) showed that although CHs maintain their overall shape over short timescales, small loops that are abundant along the boundaries continuously reconnect, changing the small-scale magnetic structure (in the order of 1-40"). Using XRT observations of coronal bright points within a $\mathrm{CH}$, Subramanian et al. (2010) showed that these small loops could be a source of slow outflowing solar wind. These loops may erupt as X-ray jets ejecting plasma along open field lines into interplanetary space. Madjarska et al. (2012) confirmed these findings using spectrograph data from SUMER/SOHO and EIS/HINDOE. In the last paper of the series, Huang et al. (2012) demonstrated that magnetic flux in $\mathrm{CHs}$ undergoes constant reconnection processes. It is suggested that these constant restructuring processes of the small-scale magnetic field within $\mathrm{CHs}$ might be largely involved with the overall magnetic flux formation within $\mathrm{CHs}$. The connection between these phenomena and their cause remains an open question.

\subsection{Progress in theory and simulations}

The lack of understanding in solar wind acceleration and solar wind structures is closely related to the problem of coronal heating. In that respect, the properties of 
the solar corona and its connection to the solar wind are not well understood. As described above, recent studies use high-resolution observations of coronal structures and underlying magnetic field in order to gain a deeper insight into the mechanisms of $\mathrm{CH}$ evolution and morphology. Remote observations are coupled with in situ measurements for investigating the impact of the solar wind structures at larger distances from the Sun. However, due to the scarce in situ measurements in interplanetary space, we need to rely on improved modeling to make more conclusive interpretations of the physical processes underlying SIRs/CIRs and their Space Weather effects.

As mentioned before, recent studies found that unipolar flux tubes, presumably the main outflow regions of the fast solar wind, cover only a small percentage of the entire area of the $\mathrm{CH}$ (see Hofmeister et al. 2017). The open flux problem in that respect is a topic on its own that is tackled by combined observational and modeling efforts.

To improve solar wind models and to ensure accurate space weather forecasting the solar wind models have to be tested and validated. The validation of solar wind models is done by comparing the simulation results to in situ measurements.

The performance assessment of the EUHFORIA solarwind model was analyzed by Hinterreiter et al. (2019). Within a thorough statistical investigation, a comparison between modeled and in situ measured solar wind highspeed streams was made to identify possible caveats of the model results. The solar wind was modeled rather well for times of solar minimum (see Fig. 34). However, during increased solar activity, complex solar-surface situations could be identified that stem from the interplay between evolving and dissipating magnetic field.

In a study by Lee et al. (2009) the heliospheric models ENLIL/MAS and ENLIL/WSA were compared with in situ measurements from ACE and Wind (time range: 2003-2006). They found that the model results give lower densities for faster solar wind fully agreeing with the solar wind momentum flux conservation. They also derived a general good agreement between the solar wind models and the in situ measurements for largescale structures and for timescales of several days. The results are in agreement with findings from Gressl et al. (2014). Jian et al. (2015) performed a comparison of several models installed at CCMC (ENLIL , MAS, WSA, SWMF) with solar wind in situ measurements and revealed strengths and weaknesses of each model. Common to all studies is the fact that different magnetogram inputs have a huge impact on the model performance. This result should be taken as basis to improve datadriven models or at least to add more observational parameters in order to better constrain the models and to identify input magnetograms that match better depending on the specific solar cycle condition.

Interesting recent studies for a better understanding of solar wind evolution are given by Jian et al. (2016) who compared ENLIL model results with observations at ACE and at Ulysses for times when ACE and Ulysses were in latitudinal alignment. The alignment made it possible to compare the model results for the same latitude due to the different radial distances (1 AU and 5.4 $\mathrm{AU})$ the evolution of the solar wind could be well observed and interpreted.

For simulating the solar wind magnetic field close to the Sun, EUHFORIA uses an adaption of the semiempirical Wang-Sheeley-Arge (WSA) model from Arge \& Pizzo (2000). WSA is composed of the Potential Field Source Surface (PFSS) (Altschuler and Newkirk 1969; Schatten et al. 1969) and the Schatten Current Sheet (SCS) (Schatten 1971). The PFSS outer boundary, called "source surface," divides the corona into an inner and outer sector. At the transition from PFSS to SCS modeling domains, artificial kinks appear in the magnetic field lines, which can be improved by putting the inner boundary of the SCS model at a distance below the source surface (McGregor et al. 2008). Presently, no exact heights for those boundaries exist, and actually varying them leads to different results in the computed open magnetic flux (Asvestari et al. 2019) (cf. Fig. 35). Moreover, as height variations of those boundaries affect the modeled magnetic field topology, e.g., the bending (spatial gradients) of the magnetic field lines, this has further effects on the computation of the expected propagation and extension of high-speed solar wind streams, and how they interact with Earth.

The magnetic topology is thought to be key, not only for the shape and morphology of $\mathrm{CHs}$ but also for the process of solar wind acceleration. In modeling, the flux tube expansion factor plays an important role in empirically determining the outflowing plasma velocities in CHs. Wiegelmann and Solanki (2004) showed that the magnetic configuration of $\mathrm{CHs}$ does not only exist of open field but is dominated by small-scale low-lying loops and few high and long. The small loops that seem to confine the expansion of the flux tubes in the transition region and lower corona were found to be on average flatter than their equivalent in the quiet sun. In that respect, the modeling of flux tubes within a $\mathrm{CH}$ is a challenge due to the much weaker magnetic field, but can be achieved as given in Fig. 36 .

\subsection{Impact at Earth}

The solar wind couples the interplanetary space with the Earth's magnetosphere. Hence, the upper atmosphere reacts to the energy input into the system depending on the speed and magnetic field of the solar wind stream. 


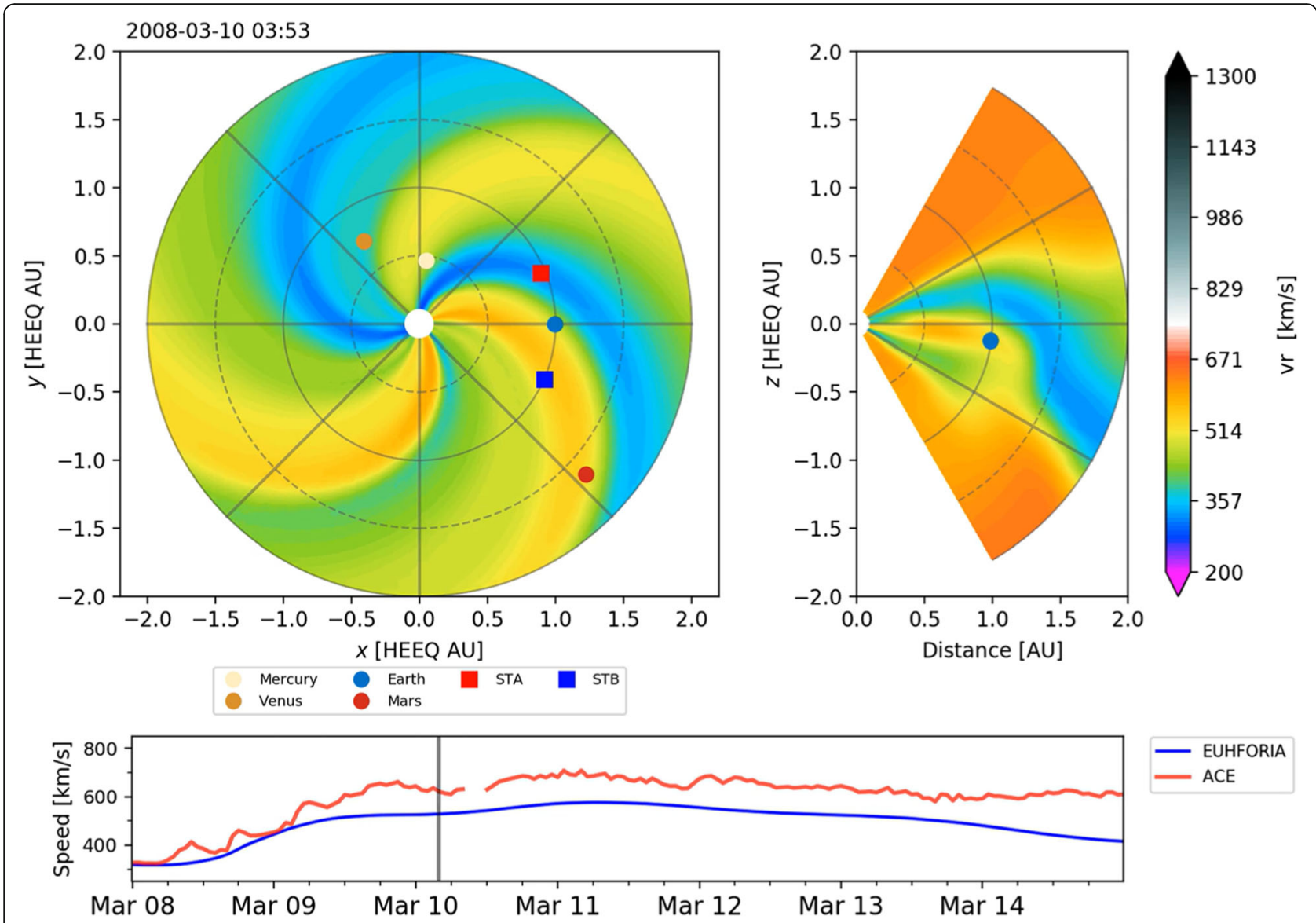

Fig. 34 Snapshot of the background solar-wind radial speed modeled by EUHFORIA. The top-left panel shows the MHD solution in the heliographic equatorial plane, and the right panel shows the meridional plane cut that includes the Earth (blue circle). The lower panel shows comparison of the modeled and observed solar wind by EUHFORIA and ACE, respectively. Taken from Hinterreiter et al. 2019

As such, especially the stream interaction regions and their compressed plasma (sometimes associated to shocks) put energy into the magnetosphere that has consequences for the upper atmosphere (coupled through Poynting flux). With the arrival of CIRs there is a persistent evolution from slow to fast solar wind and the Earth's plasmasphere significantly changes accordingly (Denton and Borovsky 2017). Spatial and temporal variations in the magnetic field are found to be most relevant for the amount of energy input into the system. Moreover, speed and density give the ram-pressure, which is well correlated to the amplitude of sudden storm commencements caused by the rapid compression of the Earth's magnetic field (Gonzalez et al. 1989). In that respect, the faster and stronger the compression, the larger the Space Weather effects. Further, the preconditioning of the magnetosphere plays an important role as pointed out in a well-cited study by Borovsky and Denton (2006). They further concluded that CIR-related storms are more hazardous to space-based assets, particularly at geosynchronous orbit compared to CMEs. The reason is that CIRs are of longer duration and have hotter plasma sheets causing a stronger spacecraft charging. Denton et al. (2016) gave an overview on unsolved problems of the magnetosphere. The atmospheric layers of the Earth all react on CIRs, and effects are measurable down to the neutral atmosphere. Variations in the thermosphere density occur in relation to the arrival of CIRs and CMEs. In that respect, CIRs and CME sheath regions have a similar impact on the amount of density increase whereas most strong variations come from the magnetic structure of the CME (e.g., Krauss et al. 2015; Krauss et al. 2018).

\subsection{Conclusions}

Studying solar wind streams and their solar sources, $\mathrm{CHs}$, is of utmost importance. The streams highly structure interplanetary space and as such interact with disturbances propagating in the flow.

Consequences for CMEs are strong changes in their propagation behavior (speed, direction) which is the primary cause of large uncertainties in the CME forecast. 

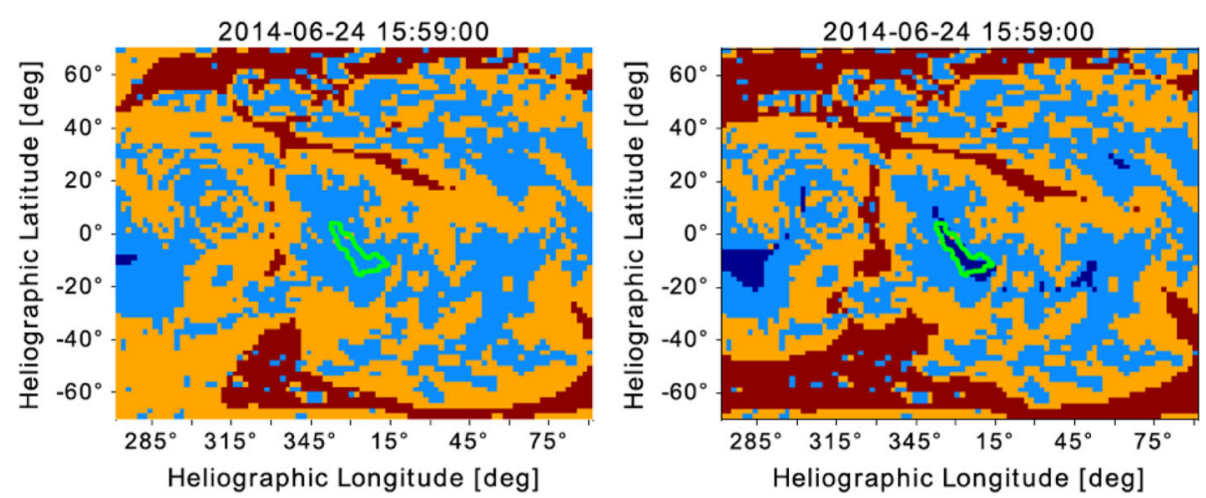

Fig. 35 Maps of open-closed flux generated for the same $\mathrm{CH}$ by two different model runs. The one on the left resulted by EUHFORIA running for the default pair of heights $[2.3,2.6]$ Rs, while the one on the right for the pair $[1.3,1.8]$ Rs. It is clear that the $\mathrm{CH}$ is not present in the model result on the left but is present and well captured by the run setup based on lower heights. This example highlights the impact the heights of the source surface and the inner boundary of the SCS model have in the modeling result. Taken from Asvestari et al. 2019

In view of this, a reliable interpretation of observed changes in the kinematical profiles of CMEs is only possible when we properly understand the variation of the ambient solar wind flow. Besides influencing near-Earth space, high-speed solar wind streams themselves are sources of geomagnetic effects and especially during times of low solar activity put with their recurrent characteristics a comparable amount of energy into the Earth atmosphere as CMEs do over short timescales.

The different atmospheric layers of the Earth are coupled through dynamical, electromagnetic, and photochemical processes. With that, geomagnetic effects due to SIRs/CIRs and CMEs may cause a cascade of impacts down to ground-level enhancements and induced currents. Long and short-term effects were studied during CAWSES that led to a significant improvement in our understanding of the solar influence on our Earth system (see special issue devoted to CAWSES-II (http:// progearthplanetsci.org/collection/001.html). SCOSTEP endorsed the continuation of the CAWSES program as CAWSES II during 2009-2013. With the success of the VarSITI program (2014-2018), the path of interdisciplinary studies will be continued in the recently approved SCOSTEP program PRESTO (Predictability of the variable solar-terrestrial coupling) that will act during 2020-2024.

\section{Forecasting CMEs}

Having discussed the observational properties, theory, and modeling of CMEs in Section 2, 3, and 4, respectively, in this section we review the efforts addressing forecasting CME occurrence (Section 8.1), time-ofarrival at $1 \mathrm{AU}$ (section 8.2), coronal and heliospheric modulations (Section 8.3) and magnetic fields (section 8.4). Recent reviews on these topics can be found in Vourlidas et al. (2019) and Kilpua et al. (2019). We will hereby mainly focus on empirical or semi-empirical

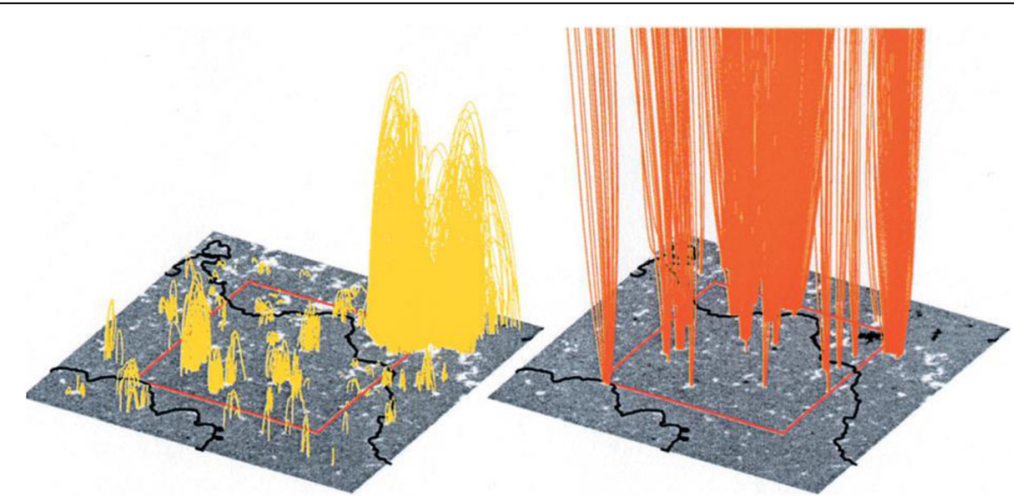

Fig. 36 Magnetic structures in a CH. The gray-coding shows the field strength in the photosphere. The black line gives roughly the boundary of the $\mathrm{CH}$. The field of view for SUMER is marked as a red rectangle in both panels. The magnetic field was constructed from a MDI magnetogram. Left figure: mostly closed loops at various scales. Only closed magnetic field lines with B $\geq 30 \mathrm{G}$ are shown. Right figure: only open fields with large photospheric values, $B \geq 100 \mathrm{G}$. The open flux is bundled in narrow uniform filaments and originates in stronger fields concentrated at small-scale footpoints. The flux tubes expand as they extend into the corona. Taken from Wiegelmann, Xia and Marsch (2005) 
physics-based approaches, which are more amenable to operational purposes in the current stage.

\subsection{Predicting CME occurrence}

The lack of critical observations (e.g., no routine observations of the magnetic field in the corona), and limitations in theory and models (e.g., idealized initial and boundary conditions), are currently not allowing to predict when a CME would occur. However, thanks to advances in our observational capacity (e.g., new observations from STEREO, Hinode, SDO, IRIS), and in modeling (e.g., increase of realism in models, dataconstrained and data-driven models) and in analysis and forecasting techniques (e.g., use of advanced statistical tools and machine learning methods) significant progress in our understanding and eventual prediction of CMEs has been achieved over the last decade.

Identifying and understanding the physical mechanism(s) behind CME onsets would be a key element in developing the capability to predict them on a regular basis. While there exists no doubt about the magnetic origin of CMEs (Forbes 2000; Vourlidas et al. 2000), there is currently no consensus regarding the specifics of the eruption process (Chen 2011; Schmieder et al. 2015; Cheng et al. 2017; Green et al. 2018; Georgoulis et al. 2019; Patsourakos et al. 2020) and sections 2 and 3.

A first approach to the prediction of the most powerful CMEs, which are in general the most geoeffective, is to use the observational finding of a rapidly increasing probability of eruption with associated flare magnitude, with flares above $\mathrm{X}$ of the GOES classification approaching 100 \% (Andrews 2003; Yashiro et al. 2005; Wang and Zhang 2007). Therefore, by assessing conditions/forecasts for major flares, one could also infer whether major CMEs could occur (Anastasiadis et al. 2017). However, the flare magnitude-CME occurrence relationship is statistical, and therefore, exceptions should be anticipated (e.g., the super-active AR 12192 which hosted 6 confined X-class (e.g., Thalmann et al. 2019). In addition, this approach excludes CMEs associated with weaker flares as well as CMEs originating from quiet Sun regions.

Eruption predictors based purely on imaging observations include SXR and EUV sigmoids (Canfield et al. 1999; Green and Kliem 2009), EUV and WL cavities (Gibson et al. 2006), EUV hot channels (Zhang et al. 2012; Patsourakos et al. 2013; Nindos et al. 2015; Cheng et al. 2017). For instance, the statistical analysis of Canfield et al. (1999) found that sigmoidal ARs are more likely to erupt than non-sigmoidal ARs. Recently, the statistical study of Nindos et al. (2020) showed that a significant fraction (i.e., two-thirds) of the erupting hot flux ropes in their sample was formed well before the actual eruptions (from $51 \mathrm{~min}$ to more than $8 \mathrm{~h}$ ) during confined flares.
These are discussed in the recent reviews by Green et al. (2018), Cheng et al. (2017), and Patsourakos et al. (2020).

Given the magnetic nature of CMEs, several magnetic metrics have been considered in the literature as eruption diagnostics. The definition of these metrics is motivated by various proposed eruption models/mechanisms and/or by physical intuition. Pertinent studies calculate a given metric for a set of eruptive and noneruptive ARs and then search whether there exist specific thresholds, or region of values of the considered metrics, segregating eruptive and non-eruptive cases. Note here, that since a small fraction of ARs gives rise to eruptive flares, this large disparity between eruptive and non-eruptive cases should be properly accounted for in the corresponding analysis.

A group of CME predictors refers to properties calculated within or around polarity inversion lines (PILs), i.e., regions in the photosphere where the vertical magnetic field changes sign. This is motivated by the fact that CMEs, and large-scale eruptive phenomena in general, originate from intense and complex PILs (Webb et al. 1997; Schrijver 2007). The corresponding studies employ line-of-sight or vector photospheric magnetograms. Various metrics are then calculated for each traced PIL and we hereby discuss a sample thereof (see also Fig. 37). Falconer et al. $(2006,2008)$ introduced several PILrelated non-potentiality measures related to for example the length of the strong shear PIL(s), the integral of the shear angle along the PIL(s), the magnetic gradientweighted integral length of the PIL(s). Considering pairs of these measures for a set of ARs, and designating suitable thresholds for each measure, supplied success rates of above $75 \%$ for an AR to give rise to a CME in the next few days from the corresponding measurements. Schrijver (2007) calculated the $\mathrm{R}$ metric, i.e., the total unsigned magnetic flux within $15 \mathrm{Mm}$ of strong-field and high-gradient PILs. He found that when an $R>2 \times$ $10^{21} \mathrm{Mx}$ is recorded, there is a high probability of a major (> M class) flare, and hence of a CME as well, within $24 \mathrm{~h}$ from the measurement. Georgoulis and Rust (2007) calculated the $B_{\text {eff }}$ metric, which essentially calculates the total magnetic field of connected magnetic partitions in strong PILs. They found that the conditional probability for the occurrence of $\mathrm{M}$ - and X-class flares within a $12 \mathrm{~h}$ window of the measurement exceeds 0.95 for $B_{\text {eff }}$ above $1600 \mathrm{G}$ and $2100 \mathrm{G}$, respectively.

Recently, Kusano et al. (2020) proposed a new physicsbased scheme (k-scheme) to predict large imminent solar flares. This scheme is based on the calculation from HMI magnetic field data and NLFF extrapolations of the critical length scale (rc), for the region of the trigger reconnection along the PIL to become unstable to the double-arc instability (Ishiguro and Kusano 2017) as 


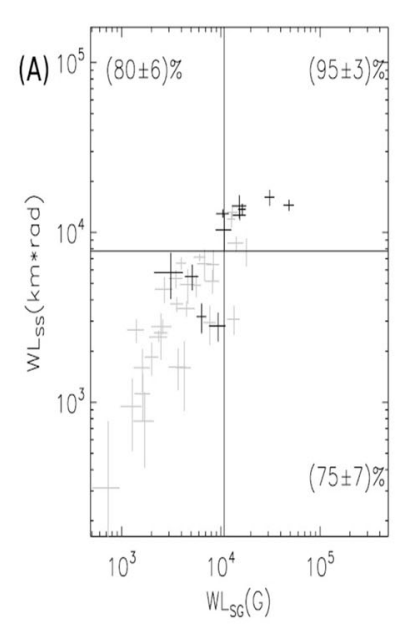

(B)
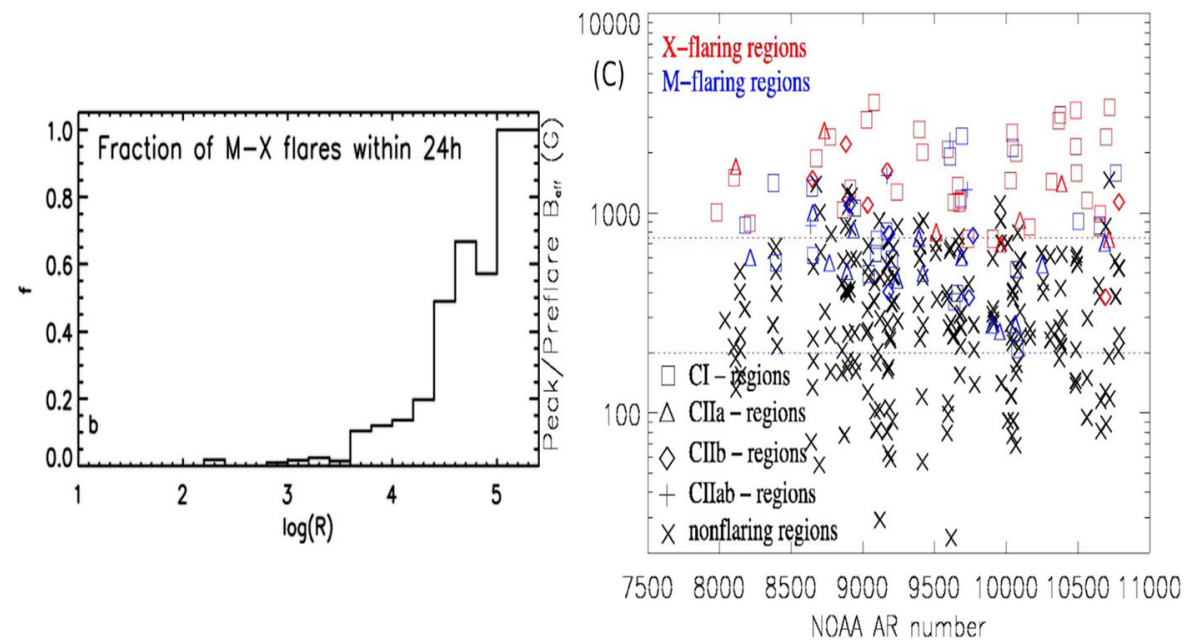

Fig. 37 Examples of magnetic metrics applied to major flares/CMEs. Panel (a): the integral of the shear angle along the PILs versus the gradientweighted integral length of the PILs for 56 bipolar and multipolar ARs. The darker crosses correspond to ARs which gave rise to a CME within the $72 \mathrm{hr}$ search window from the corresponding measurement whereas the lighter crosses correspond to those ARs without a CME with the same search window. From Falconer et al. (2008). Panel (b): fraction of ARs with an M- or X-class flare as a function of R, within a 24-hour window from the R recording. 2500 ARs were employed. From Schrijver (2007). Panel (c): ARs giving rise to M- or X-class flares (blue and red symbols) and nonflaring ARs (black symbols) versus their peak $B_{\text {eff }}$ in a 24-hour search window. 298 ARs were employed. From Georgoulis and Rust (2007)

well as the magnetic field energy (Er) of the field overlying the double-arc loop. Kusano et al. (2020) showed for solar cycle 24 that in 6 out 7 ARs giving rise to major eruptive flares (X2 class and above) had $\mathrm{rc}<1 \mathrm{Mm}$ and Er $>4 \times 10^{31}$ ergs along their PILs and these conditions were satisfied for periods ranging from several hours up to one day before the onset and indeed k-scheme represent a showcase of how MHD theory could be utilized in the prediction of eruptive flares. Kontogiannis et al. (2019) used a number of properties of eruptive ARs and found that the total amount of their non-neutralized electric currents and the length of their main polarityinversion lines have the strongest correlation with the associated CME properties, and therefore these two parameters better reflect the potential of an AR to produce eruptions.

Magnetic helicity (e.g., review by Pevtsov et al. 2014), a measure of the twist and linkage of magnetic field lines, is an extensively employed quantity given also its conservative nature (e.g., Berger and Field 1984) which allows to draw links between CMEs in the Sun and in the interplanetary medium (Kumar and Rust 1996; Green et al. 2002; Démoulin et al. 2002). In addition, it plays a central role in models of filament channel formation such as the helicity condensation model (Antiochos 2013). There exist several methods to calculate the magnetic helicity in the corona or its injection rate in the photosphere. Statistical surveys supplied important clues on the importance of magnetic helicity in CMEs. Park et al. (2008) found that the accumulated magnetic helicity prior to a set of $11 \mathrm{X}$-class flares over intervals half a day up to a few days was significant and in the range $1.8-32 \times 10^{42} \mathrm{Mx}^{2}$. Nindos and Andrews (2004) studied the pre-flare helicity of active regions which gave rise to big flares, and showed, that statistically speaking active regions with eruptive flares are associated with larger magnetic helicity compared to active regions with confined (i.e., non-eruptive) flares LaBonte et al. 2007. found that active regions giving rise to $\mathrm{X}$-class flares, hence with a high probability of being eruptive, exhibit peak helicity flux injection rates above $6 \times 10^{36} \mathrm{Mx}^{2} / \mathrm{s}$ in 24hour windows prior to the considered flares. Tziotziou et al. (2012 found that helicity and magnetic free energy thresholds of $\sim 2 \times 10^{42} \mathrm{Mx}^{2}$ and $\sim 4 \times 10^{31}$ ergs separate eruptive with non-eruptive ARs. Using MHD simulations, Pariat et al. (2017) suggested that the ratio between the current-carrying part of magnetic helicity to the total (volume-integrated) helicity could be used as a discriminator between eruptive and non-eruptive cases. This has been recently tested in a handful of cases (Moraitis et al. 2019; Thalmann et al. 2019), where it was found that this ratio increases significantly prior to eruptive flares while it does not significantly change prior to confined flares. The recent study of Pagano et al. (2019) showed that a metric based on the calculation of the Lorentz force from data-driven nonlinear forcefree field models could help in discriminating between eruptive and non-eruptive active regions.

Another important physical parameter pertinent to eruptivity is the decay index $(n)$ of the overlying horizontal magnetic field. This essentially measures how fast the strapping magnetic field declines with height above 
the erupting flux. Its value assumes a key role in certain models such as the torus instability (Kliem and Török 2006). Depending on the properties of the magnetic setup (e.g., bipolar, multipolar, aspect ratio and shape of flux rope, etc.), $n$ should be at least as steep as $~[0.5,2]$ for an eruption to take place (Kliem and Török 2006; Fan and Gibson 2007; Démoulin and Aulanier 2010; Olmedo and Zhang 2010; Zuccarello et al. 2016; Syntelis et al. 2017). Note that stability against the torus instability could become more involved for complex systems like multiple flux ropes (Inoue et al. 2016). Magnetic field extrapolations are normally employed in the calculation of the coronal magnetic field in regions that erupted (or not). In some cases, the starting height of the eruption, as inferred from height-time single or multi-viewpoint measurements, is used as the bottom boundary for the decay-index analysis.

The statistical study by Liu (2008) showed that the difference of $n$ between eruptive (>1.74) and non-eruptive cases $(<1.71)$ is statistically significant. Analysis of $n$, for a set of confined and eruptive flares which took place in the same active region, showed that the eruptive flares were associated with steeper $n$ in the low corona compared to the confined ones (Cheng et al. 2011). The statistical studies of Wang et al. (2017) and Baumgartner et al. (2018) showed that the critical $n$ (i.e., 1.5), as per Kliem and Török (2006), is achieved at somehow larger heights above active regions which gave rise to confined flares as compared to cases associated with eruptive flares. Surveys of eruptive prominences by McCauley et al. (2015) and Vasantharaju et al. (2019) found $n$ in the range $[0.8,1.3]$ above the starting heights of the eruptions. Duan et al. (2019) calculated $n$ along inclined paths, reflecting non-radial CME propagation rather along the local vertical, and found that all eruptive flares they considered had $n>1.3$. Recently, Cheng et al. (2020) found that the average $n$ at the onset heights of the main acceleration was close to the torus-instability threshold for the 12 CMEs they analyzed. The segregation between eruptive and non-eruptive cases in terms of $n$ is also reported in several case studies as discussed in the review of Cheng et al. (2017). However, taking into account the temporal evolution of $n$ seems that a steep decay index of the overlying magnetic field is a necessary but not a sufficient condition for eruptions to take place (Nindos et al. 2012; Suzuki et al. 2012; Chintzoglou et al. 2015). In addition, cases of high-lying QS filaments with overlying magnetic fields with $n>1.5$ associated with confined eruptions could be also found (Zhou et al. 2019). Strong overlying fields seem to prevent CMEs as vividly illustrated in the case of superactive AR 12192 which exhibited a multitude of confined flares, including X-class (Chen et al. 2015; Sun et al. 2015).
The twist number (Tw) of magnetic field lines is another parameter that is extensively used in CME onset studies (e.g., Inoue et al. 2011). The magnetic twist is an integral part of magnetic helicity discussed above, and a comparison of its properties/distribution in the preeruptive/eruptive configurations in the solar atmosphere and at 1 AU supplies important physical clues about CMEs (Wang et al. 2016d). In addition, magnetic twist plays a key role in the triggering of the helical kink instability, and related Tw thresholds in the range $\sim[1.25$, 2.0], depending on the specifics of the implementation (e.g., twist profile, employed geometry, etc.), were derived (Hood and Priest 1981; Fan and Gibson 2003; Török and Kliem 2003; Török et al. 2004; Hassanin and Kliem 2016). Jing et al. (2018) calculated the spatial average of $\mathrm{Tw}$ for a sample of 38 eruptive and confined flares. They found that Tw was not playing a role in separating between confined and eruptive flares, by indeed all eruptive cases having Tw smaller than the lower bound of the Tw kink instability threshold (i.e., 1.25) discussed above. On the other hand, a similar survey of 45 eruptive and confined flares by Duan et al. (2019), calculating this time the maximum $\mathrm{Tw}$ per flux rope, showed that $\mathrm{Tw}$ above (below) 2, i.e., close to the upper bound of the kink-instability thresholds discussed above, were relevant to the majority of the considered eruptive (confined) flares, therefore allowing to segregate between confined and eruptive cases. The differences between these two studies could be possibly attributed to the different approaches used to calculate the coronal magnetic field and Tw, i.e., NLFFF extrapolations and average Tw in Jing et al. (2018) and magnetic relaxation and maximum Tw in Duan et al. (2019). Recently, Lin et al. (2020) proposed a new parameter (rm) that could be used in eruptive flare prediction. It is based on the ratio of the magnetic flux of twist higher than a threshold over the overlying magnetic flux and discriminant function analysis showed that $\mathrm{rm}$ is moderately able to tell ARs which are capable of producing eruptive flares. Irrespectively of whether kink instability assumes the main role in setting CMEs, it may still, in cases of confined eruptions triggered by this instability, lift flux ropes to heights where the torus instability could take over.

The existence of coronal null points (i.e., points of vanishing magnetic field) has a central role in CME models such as the breakout model (Antiochos et al. 1999). Therefore, magnetic field extrapolations are used to investigate whether coronal null points exist above erupting ARs, e.g., Aulanier et al. (2000) for the first such application. Searches of coronal null points and their association with CMEs have been extended to larger statistical samples by Ugarte-Urra et al. (2007) and Barnes (2007). These studies found that a significant fraction of the analyzed ARs with 
coronal null points were eruptive, $73 \%$ and $26 \%$, respectively. Both studies reported that for the majority of the considered eruptive cases ( 75\%), no preeruptive coronal null points were found.

Syntelis et al. (2016) found a rise in the emission measure for hot plasmas $(T>6 \mathrm{MK})$ before two eruptive flares and this was extended to 16 ARs by Gontikakis et al. (2020) who showed that the positive temporal derivative of the total emission measure could conceivably be used as a credible precursor, or short-term predictor, of an imminent major flare.

As discussed in the introduction of this section, the problem of predicting major CMEs could be mitigated by predicting major flares. Flare forecasting is a field that has really boomed over the last decade because of the quantum increase of vector magnetic field data, thanks to HMI on SDO, and the advent of machine-learning (ML) in heliophysics. ML schemes allow to digest in autonomous or semi-autonomous means large volumes of data, explore multi-dimensional parameter spaces, and are particularly suited for identification and classification tasks (e.g., review of Camporeale (2019) of ML applications in heliophysics. Recent reviews on flare forecasting, including ML schemes, could be found in Leka et al. (2019a), Leka et al. (2019b), Park et al. (2020)). Major conclusions from their extensive benchmarking of a large number of methods currently used in flare forecasting are that such numerous methods do better than climatology, no method clearly outperforms the others, and consideration of prior flare history improves the corresponding skill scores.

ML has been used directly in CME predictions. Bobra and Ilonidis (2016) applied a Support Vector Machine classification scheme to 18 parameters derived from HMI vector magnetograms for more than 3000 ARs and found that only a handful (i.e., 6) among these parameters is sufficient to separate erupting and non-erupting ARs within 24 hours from the corresponding measurements. These parameters (e.g., mean gradient of the horizontal magnetic field, mean current helicity, mean twist parameter) are intensive (i.e., do not depend on the AR size but are spatial averages) and not extensive (i.e., depend on the AR size and correspond to spatial sums). Interestingly, extensive measures seem more appropriate for the prediction of any flare, irrespectively of its eruptivity (e.g., Bobra and Couvidat 2015).

MHD simulations are invaluable in evaluating existing metrics as well as for supplying physical insight into new metrics. For example, Guennou et al. (2017) analyzed a set of eruptive and non-eruptive MHD simulations and found, in agreement with the ML work discussed in the previous paragraph, that intensive parameters are more relevant to eruptivity. Another example is the study of Moraitis et al. (2014) who validated the existence of particular magnetic helicity-free magnetic energy regimes pertinent to eruptivity as reported in the observational study of Tziotziou et al. (2012). Routine observations of the AR vector magnetic field at several layers above the photosphere may be instrumental in predicting CMEs (e.g., Patsourakos et al. 2020).

\subsection{Predicting CME time of arrival}

The essential questions regarding the CME forecast are if and when it will hit Earth. Therefore, various models and methods have been proposed in the past decades to give (a reliable) answer to these questions. Recently, Vourlidas et al. (2019) gave an extensive overview of the time-of-arrival (ToA) forecast models, whereas earlier reviews include that of Zhao and Dryer (2014) and Siscoe and Schwenn (2006). In this review, ToA models are noted and described in Sections 3 (analytical and semiempirical models) and Section 4 (numerical models), whereas in this section, we will primarily focus on their performance.

Vourlidas et al. (2019) made a comprehensive summary of the mean absolute error (MAE) reported by numerous studies and found that the unweighted mean of all MAE is $9.8 \pm 2 \mathrm{~h}$, representing the value for the current state of accuracy of ToA studies. However, as noted by Vourlidas et al. (2019) and Verbeke et al. (2019), most of the studies on the ToA prediction does not report their performance validation consistently, and moreover, a comparison of the ToA performance between different methods/models is generally missing. Not only should different methods be compared on the same sample and under the same conditions, but also community-agreed metrics and validation methods should be used in order to assess the current state of CME modeling capabilities unbiasedly. An effort in that direction was recently made by Riley et al. (2018b), who compared the performance of different methods and models that performed predictions on the CME scoreboard (https://kauai.ccmc.gsfc.nasa.gov/CMEscoreboard/ ). The CME scoreboard, facilitated by the Community Coordinated Modeling Center (CCMC), is one aspect of the efforts of the CME Arrival Time and Impact Working Team that started in 2017 in the scope of the community-wide International Forum for Space Weather Capabilities Assessment. Riley et al. (2018b) explored the accuracy and precision of the predictions made by 32 teams 2013-2017 and found that the models on average predict arrival times to within \pm 10 hours with the precision around the average of \pm 20 hours. In addition, they found that the "Average of all Methods" forecasts generally perform as well as, or outperforms, the other models, thus acting as a simple superensemble approach. It should be noted that the ensemble approach was implemented in several ToA models/ 
methods in the past years (Mays et al., 2015a; Dumbović et al., 2018a, b; Amerstorfer et al. 2018; Kay and Gopalswamy 2018; Napoletano et al. 2018), as it can provide a probabilistic forecast of CME arrival time as well as an estimation of arrival time uncertainty from the spread.

The current state of the accuracy of ToA prediction, regardless of the method used, seems to revolve around 10 hours. This resemblance in the performance of very different propagation models indicates that the major drawback lies in the lack of reliable observation-based input. This includes the CME input parameters as well as the input of the heliospheric background in which it propagates, which on their own contain errors. Namely, to obtain CME input parameters, such as the CME velocity and angular width, different methods and models have to be used to go past the problems related to the projection effects (for observational properties of CMEs see Section 2). Different methods can present a rather wide spread in the obtained CME parameters on a case by case basis (Mierla et al. 2010). Therefore, it is important to keep track of the CME parameter measurement metadata and to test the CME input errors. On the other hand, the model performance also depends on the input of the heliospheric background, which hugely relies on our current capabilities of solar wind modeling (see Section 4). Recently, Kay et al. (2020a) performed an analysis of the ToA sensitivity of arrival times to various input parameters for drag-based models. They found that the ToA tends to be more sensitive to CME parameters than solar wind parameters and that different precisions on the input parameters are needed for different "strength" CMEs. We note that improvements in the accuracy of CME ToA prediction may be achieved using novel approaches, e.g., assimilation of interplanetary scintillation observations demonstrated by Iwai et al. (2021). We expect more such studies will be performed in the near future and especially in the scope of the new SCOSTEP program PRESTO.

\subsection{Predicting the magnetic field of CMEs}

Predicting the magnetic field distribution within CMEs/ ICMEs represents a holy grail in heliophysics, given the fact that extended intervals of intense southward magnetic fields, typically associated with ICMEs, spawn the stronger geomagnetic storms (e.g., Gonzalez et al. 1999). A thorough account of the state-of-the-art in this important problem was recently given in (Vourlidas et al. 2019). Currently, we are able to routinely observe the magnetic field of CMEs only at the "end of the road" to geospace, via in situ observations at 1 AU by the WIND, ACE, etc. spacecraft (e.g., Chi et al. 2016; Nieves-Chinchilla et al. 2019 and Section 3). Occasionally we can observe it also in the inner heliosphere beyond $\sim 0.3 \mathrm{AU}$, with HELIOS (e.g., Bothmer and Schwenn 1998), and more recently with Messenger and VEX (Miho Janvier et al. 2019; Good et al. 2019). Much closer to the Sun, in the corona, we have only a few reported cases of direct observations of the magnetic field of CMEs in the radio domain. These observations exploit gyrosynchrotron emissions from mildly relativistic electrons spiraling in CMEs (Bastian et al. 2001; Tun and Vourlidas 2013; Carley et al. 2017; Mondal et al. 2020) and Faraday rotation of the electromagnetic radiation of either natural (e.g., pulsars) or artificial sources going through the CME body (Jensen and Russell 2008; Howard et al. 2016; Kooi et al. 2017, 2021). The lack of continuous monitoring of the Sun with solar-dedicated instruments and the low sensitivity of the existing instrumentation is behind the scarcity of radio diagnostics of CME magnetic fields.

Therefore, we have to mainly rely on modeling, either MHD or empirical/semi-empirical, to remedy this critical deficiency. MHD simulations of CMEs covering the domain spanning the lower solar atmosphere, the outer corona, and the inner heliosphere out to $1 \mathrm{AU}$, could in principle deal with the problem in a self-consistent manner, since they simultaneously treat CME initiation, evolution, and propagation in a realistic background corona and solar wind (e.g., Jin et al. 2017b; Török et al. 2018 and review by Manchester et al. 2017). However, such simulations, given the huge resources they require, are currently used almost exclusively for research purposes and not for forecasting.

A more tractable approach and recently developed capability is to use heliospheric models of magnetized CMEs (see Section 4.2 for details). These models represent a major step over heliospheric CME models which treat CMEs as purely hydrodynamic disturbances such as the widely-used ENLIL model (Odstrcil et al. 2004), which is indeed the standard operational space weather model (Mays et al. 2015a). Heliospheric CME models launch CMEs in their inner boundary, typically in the range 10-20 Rs, and follow their evolution in the inner heliosphere (e.g., Odstrcil et al. 2004; Shen et al. 2011a, b, c; Shiota and Kataoka 2016; Verbeke et al. 2019a; Scolini et al. 2019b; see also Section 4). The prescribed CMEs are empirically constrained by STEREO observations supplying their speed, size, and orientation. Magnetized CME models require also inputs for the CME magnetic field in the inner boundary of their computational domain such as the axial magnetic field. The less computing resources that these models require allows to run them in almost real time, with ensemble studies testing the influence of uncertainties of the input parameters on the ICME properties upon impact at $1 \mathrm{AU}$.

To deal with the lack of direct routine observations of the near-Sun magnetic field of CMEs, an ever-growing number of empirical or semi-empirical models to infer this vital parameter has appeared over the last decade 
(Vourlidas et al. 2000; Kunkel and Chen 2010; Savani et al. 2015, 2017; Isavnin 2016; Patsourakos et al. 2016; Patsourakos and Georgoulis 2017; Möstl et al. 2018; Sarkar et al. 2020). These models can be used to supply inputs to the heliospheric magnetized CMEs' models, and to predict on their own CME magnetic profiles at $1 \mathrm{AU}$. Various empirical inputs (e.g., CME positional, orientation, and size information, reconnected magnetic flux and SXR light-curve of the associated flare, eruptionrelated magnetic helicity, height-time measurements of the CME, CME energetics, etc.) and underlying assumptions (conservation of energy, magnetic flux, and magnetic helicity, self-similar expansion, force-free/nonforce-free magnetic fields, etc.) are used. The handedness of the near-Sun CME flux ropes is derived from empirical schemes based on, for example, the CME source region hemisphere, the sense of winding of observed features, etc. (Bothmer and Schwenn 1998; Palmerio et al. 2017). Given that the inputs to these models could be retrieved from easy-to-obtain observations from magnetographs, imagers, and coronagraphs, and their analytical or semi-analytical nature, they could be used to routinely infer the near-Sun magnetic field of CMEs and to predict it upon impact at 1 AU. In addition, ensemble studies to account for uncertainties in the inputs are feasible at a minimal computational cost. Meaningful and rigorous comparisons between forecasts and observations could benefit from tools/concepts developed for terrestrial weather (Austin and Savani 2018). A major conclusion was that while existing models supply encouraging results, they nevertheless require further development and validation. For instance, there exist models that lack the ability to derive beforehand the near-Sun CME magnetic field and have to rely on the in situ observations upon the corresponding ICME arrival at $1 \mathrm{AU}$ in order to properly scale the predicted CME magnetic field vectors at $1 \mathrm{AU}$. In addition, there is no benchmarking of these models. A zero-order comparison of the near-Sun CME magnetic fields from two of these models seems encouraging (see Fig. 38).

Another major limiting factor in our ability to forecast CME magnetic fields upon impact at $1 \mathrm{AU}$ is related to the uncertainties of the input parameters of the employed models. This includes uncertainty related to the deflection and rotation that CMEs undergo, in the determination of the initial (i.e., source region) and coronal CME location and orientation. In addition, models also rely upon the properties of the background corona and solar wind, which are derived from different models/approaches, which constitutes another source of uncertainty. A common outcome of these studies, whether based on Sun-to-Earth MHD simulations (e.g., Török et al. 2018), heliospheric MHD models (e.g., Verbeke et al. 2019a; Scolini et al. 2019c), or semi-analytical physics-based models (e.g., Kay et al. 2017, 2020b) is that rather small changes in the CME positional and orientation parameters in the range $2-20^{\circ}$, could have a significant impact on the predicted CME magnetic field profiles at $1 \mathrm{AU}$, and particularly on the field components (e.g., Fig. 39). Pattern recognition applied to in situ observations of incoming ICMEs at 1 AU could be also used to predict CME magnetic fields with however much shorter lead times of a few hours only (Chen et al. 1997b; Riley et al. 2017; Salman et al. 2018; Camporeale 2019).

Very recently, important results regarding the nature of young CMEs started to emerge from the first observations of the recently launched PSP mission (NievesChinchilla et al. 2020; Hess et al. 2020). As more CMEs are observed by PSP, as well as by the SoLO mission, it should be possible to validate and to eventually increase the physical realism of models of CME magnetic field forecasting. In situ monitoring of ICMEs at Venus orbit tied with either empirical scaling laws and/or propagation models could supply predictions of Earth-bound CMEs with a lead-in of $\sim 1$ day prior to impact (Kubicka et al. 2016). Recent or upcoming facilities observing in radio like LoFAR, MWA, and SKA will finally supply more systematic observations of CME magnetic fields in the corona (e.g., Nindos et al. 2019).

\section{Minimax24 project}

The ISEST/MiniMax24 non-flare target is an email alert service about non-flare-related, but possibly geoeffective, phenomena provided daily by the observer on duty. The observation overview is shown in Fig. 40. The service was first established in the scope of the SCOSTEP/CAWSES "MiniMax24 Campaign" in 2013, which was declared as the year of "MiniMax24" to note that, even though the Sun is going through activity maximum conditions, the activity is rather low. The goal of the action was to understand and explain the current behavior of the Sun and its potential impact on human society and Earth's space environment through yearlong scientific and outreach activities. The campaign team counted 37 institutions from 17 countries focused on the solarterrestrial observations of solar eruptive events through the MaxMillenium program of solar flare research (http://solar.physics.montana.edu/max_millennium/) as well as $\mathrm{CHs}$, filaments, and CIRs (i.e., non-flare related phenomena) through the newly established email alert service. By the end of the year of "MiniMax24," MiniMax24 email list reached more than 140 participants from more than 30 countries. As the MiniMax24 has shown to be a very useful and successful hub for the scientific community, the action transcended from its original 1-year-campaign scheme and was included in the 


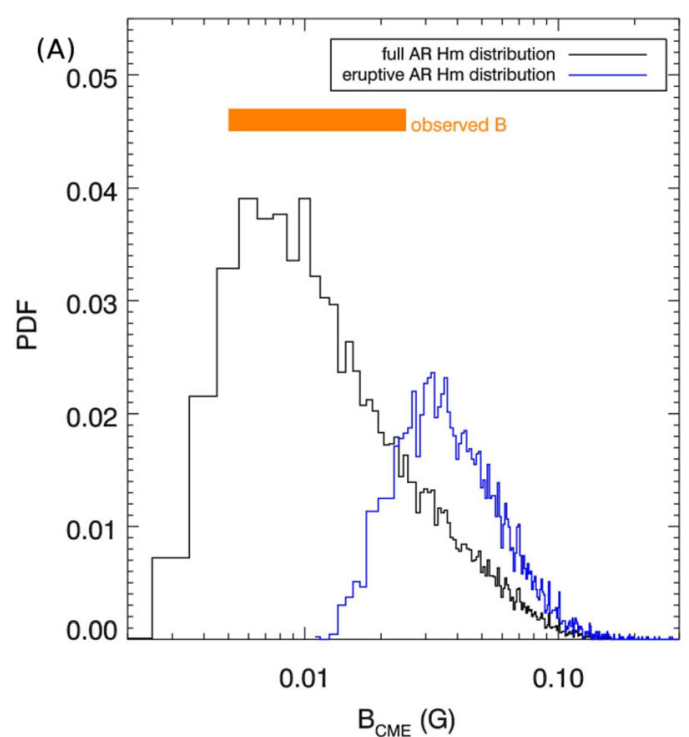

(B)

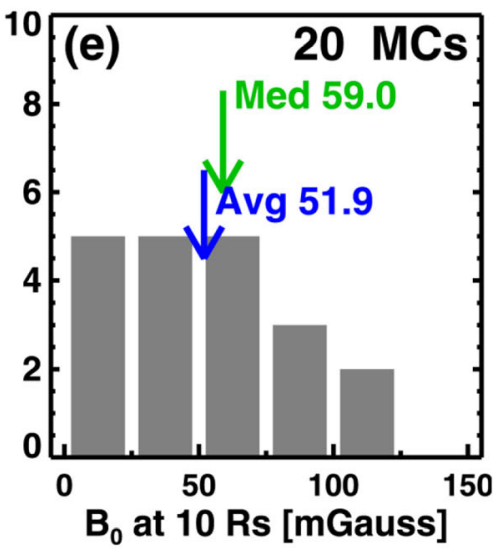

Fig. 38 Histograms of near-Sun CME axial magnetic fields at 10 Rs from two different methods. Panel A. From Patsourakos \& Georgoulis (2017) (blue histogram corresponds to eruptive cases). Panel B. From Gopalswamy et al. (2018c) (results from CMEs corresponding to magnetic clouds upon impact at $1 \mathrm{AU})$

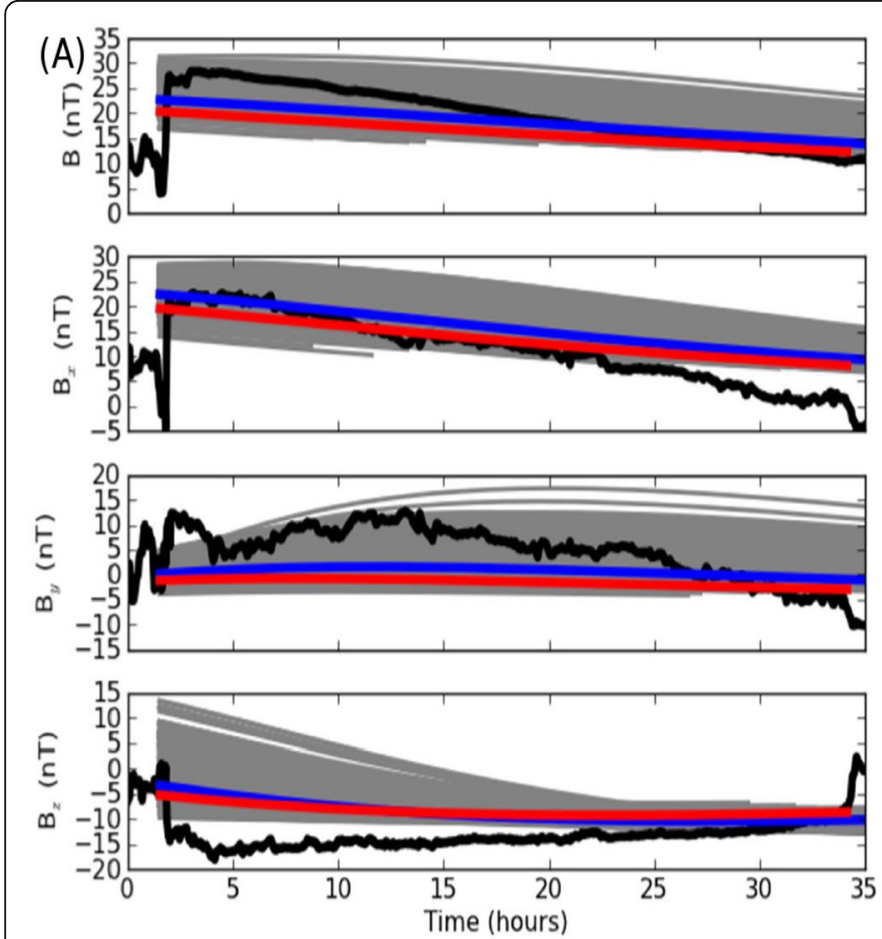

(B)
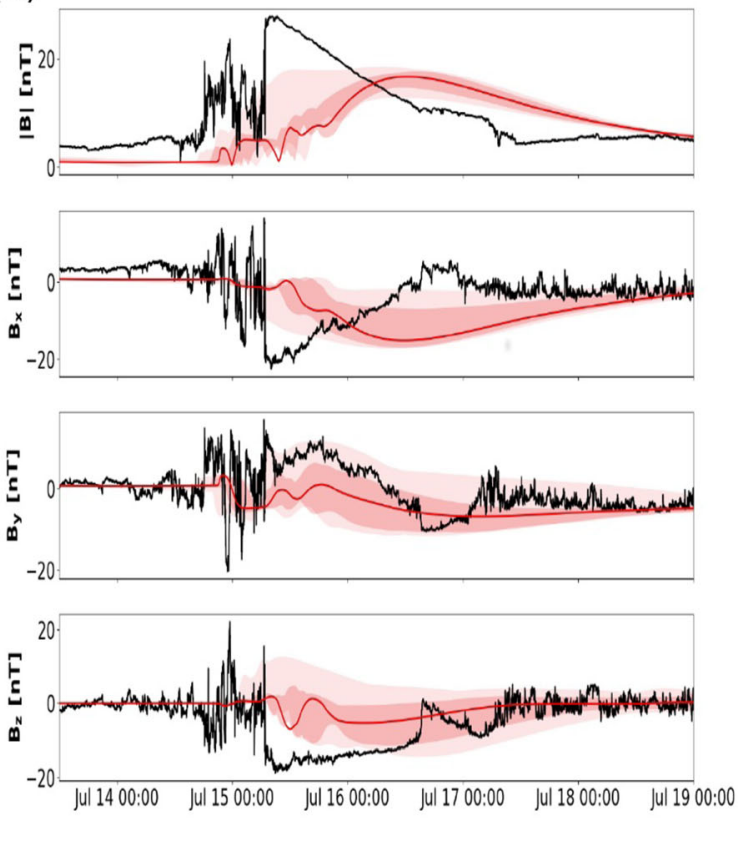

Fig. 39 Predicted (non-black curves and shaded areas) and observed (black curves) CME magnetic field profiles at 1 AU from two different models. Panel (a): from Kay and Gopalswamy 2018; panel (b): from Scolini et al. 2019b. The predicted profiles consider uncertainties in input parameters. In both panels from top to bottom the magnetic field magnitude, and its Bx, By, and Bz components in the GSE system are plotted 


\section{OBSERVATION OVERVIEW}

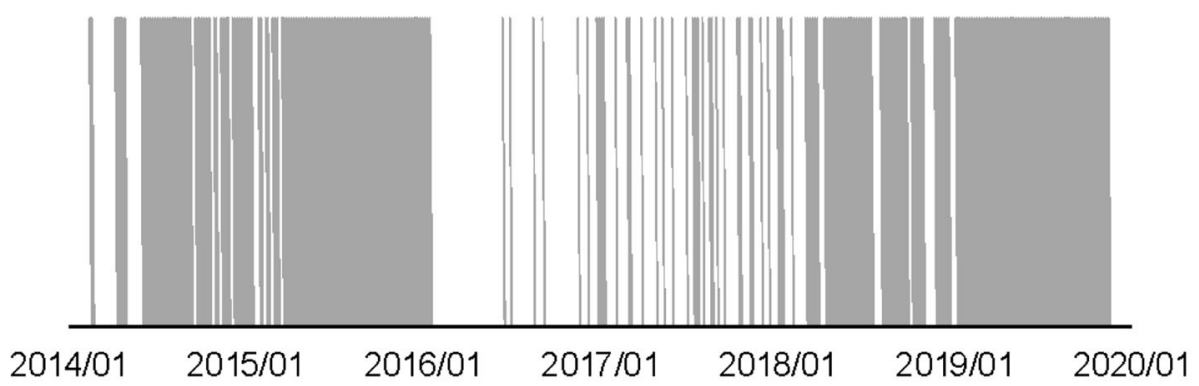

OBSERVING TIME

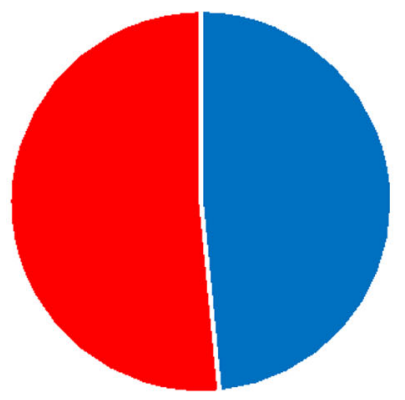

- non-observation days

- observation days

\section{ACTIVITY IN OBSERVED DAYS}

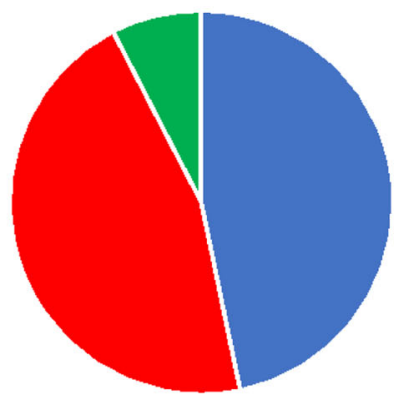

- no activity observed

- filam ent observed

- $\mathrm{CH}$ observed

Fig. 40 On the top: archived non-flare target alerts 2014-2020; Bottom left: number of archived alerts (observation days) vs number of nonarchived alerts (observation days); bottom right: number of quiet days, filament, and $\mathrm{CH}$ observations in the archived alerts

new SCOSTEP program VarSITI, as one of the working groups of the ISEST project.

The aim of the ISEST/MiniMax24 non-flare target is to monitor and warn against potentially geoeffective phenomena that are not related to solar flares. This includes CMEs that are not accompanied by solar flares, but rather eruptive filaments, as well as stream interaction regions associated with coronal holes.

\subsection{The scientific outline}

CMEs may either originate from strong active regions, large-scale relatively weak magnetic fields, or filaments (Green et al. 2018). The production of CMEs is highly likely when associated with eruptions of filaments Schmieder et al. 2013 and eruptive filaments are often used as on-disk signatures of the non-flare related CMEs. These do not show significantly different properties from the flare-related CMEs (Vršnak et al. 2005; Chmielewska et al. 2016), unlike, e.g., "stealth CMEs," i.e., CMEs without obvious on-disk signatures, which are slower and therefore potentially less geoeffective
(Robbrecht, Patsourakos and Vourlidas 2009; Kilpua et al. 2014; Nitta and Mulligan 2017). Therefore, eruptive filaments can be regarded as potential sources of significant geoeffective events. Since filaments are regarded as cool plasma suspended in the magnetic dips of the flux rope (Gibson 2018), the scale of the filament is directly related to the scale of the flux rope, and therefore, by that logic, large and dark filaments are indicative of large flux ropes, i.e., more massive/energetic CMEs, which are then more likely to be significantly geoeffective (Gopalswamy et al. 2007). Thus, the ISEST/MiniMax24 focuses on detecting and monitoring only large and dark filaments. Since the CMEs are largely propagating radially, those originating from sources close to the center of the solar disc are more likely to arrive at Earth and, therefore, more likely to be geoeffective (Srivastava and Venkatakrishnan 2004; Gopalswamy et al. 2007; Zhang et al. 2007). Therefore, the ISEST/MiniMax24 focuses on filaments located close around the central meridian. It should be noted though, that not all filaments erupt and that filament eruptions are not necessarily related to CMEs and might 
be triggered by, e.g., magnetic flux emergence or local and large-scale photospheric motions (Parenti 2014). From that perspective, ISEST/MiniMax24 only alerts on the possibly geoeffective filament targets.

Another significant source of non-flare-related geoeffectiveness, as pointed out in Section 7, are SIRs formed by the interaction of the high-speed solar wind originating from a $\mathrm{CH}$ with the preceding slower solar wind (Richardson 2018). CHs are the darkest and least active regions of the Sun, associated with rapidly expanding open magnetic fields and acceleration of the solar wind (Cranmer 2009) and can be easily outlined automatically in EUV images using the threshold technique (Rotter et al. 2012). Moreover, the area of the $\mathrm{CH}$ was found to be highly correlated with the speed of the corresponding HSS at Earth (Nolte et al. 1976; Tokumaru et al. 2017) and was found to typically need about 4 days to arrive at Earth (Vršnak et al. 2007a, b; Temmer et al. 2007). Since generally geoeffectiveness is related to the dawn-to-dusk electric field and therefore solar wind flow speed (Richardson and Cane 2011), the ISEST/MiniMax24 relies on the premise that the potentially geoeffective HSS emanate from large $\mathrm{CHs}$ close to the central meridian. Empirical relations between coronal holes and HSS have been utilized to produce a tool for automatic detection of the $\mathrm{CH}$ area in a meridional slice around the center of the solar disc to predict solar wind speed near Earth 4 days in advance (Vršnak et al. 2007a, b; Rotter et al. 2012; Reiss et al. 2016). This HSS forecast algorithm is called "Empirical Solar Wind Forecasting" (ESWF) tool and it operates automatically using near real-time SDO/AIA $193 \AA$ images to forecast solar wind speed 4 days in advance (http://cesar.kso.ac.at/programme/minimax.php).

\subsection{Non-flare target alert description}

The non-flare target alerts are sent on a daily basis, with a "NO non-flare targets" descriptor for quiet times and brief notification and description of the activity for nonquiet times. The non-quiet times are defined based on the following criteria:

Criterion \#1. There is a $\mathrm{CH}$ located within $+/-7.5^{\circ}$ in longitude exceeding a ratio area of 0.2 , or predicted SW speed at Earth exceeding $500 \mathrm{~km} / \mathrm{s}$ as observed/forecasted by the ESWF tool.

Criterion \#2. There is a prominent (i.e., dark and wide) filament located in longitude within $+/-30^{\circ}$ around the central meridian as detected in $\mathrm{H}$-alpha images provided by Kanzelhöhe Observatory or GONG $\mathrm{H}$-alpha network (http://halpha.nso.edu). If one or more $\mathrm{CHs}$ are identified as non-flare targets based on criterion 1 , the observer notifies in the alert the total ratio area (calculated cumulatively for all $\mathrm{CHs}$ across the whole meridional slice), approximate position of each $\mathrm{CH}$, and the arrival time and speed of the corresponding HSS. If one or more filaments are identified as non-flare targets based on criterion 2, the observer notifies in the alert the position, E-W and N-S spread of each filament. In both cases, the reference to the observing image is provided. The email alerts were not systematically stored until 2018, although some of the observers archived a significant part of the alerts. Since the beginning of 2018, the alerts are systematically archived and together with previously stored alerts compiled into a single nonflare target catalog maintained by the ISEST/MiniMax 24 team and available upon request (contact email: mateja.dumbovic@geof.unizg.hr).

\subsection{Summary}

The ISEST/MiniMax24 non-flare Target activity started as a 1-year SCOSTEP/CAWSES activity and continued throughout the SCOSTEP/VarSITI program in the scope of the ISEST project. It has been shown that it is a useful scientific community service that provides a daily overview of the non-flare solar activity. Moreover, as an added value we highlight that this activity has significantly helped to improve the visibility of the young scientists acting as the daily observers. Therefore, the MiniMax24 activity is planned to continue within the new SCOSTEP program PRESTO, in the scope of which it is in addition planned to archive and compile all the alerts into a non-flare target catalog to be used for future statistical analysis.

\section{Conclusion and outlook}

The aim of the ISEST project is to understand the origin, propagation, and evolution of solar transients through the space between the Sun and the Earth and develop the prediction capability for space weather with particular emphasis on the weak solar activity prevailing in solar cycle 24 . The ISEST project dealt with shortterm solar variability in the form of flares, CMEs, and SIRs and the associated phenomena such as interplanetary shocks and SEP events by forming several working groups to focus on specific problems. The MiniMax24 program, which started during the previous SCOSTEP program, has proven to be extremely useful. This program will be continued and become a permanent feature. The program has developed an extensive database on Earth-affecting transients that is available to the scientific community (Fig. 40). The ISEST program also brought together hundreds of scientists from all over the world to focus on specific problems that resulted in rapid progress. In the following, we discuss the implications of the current status and suggestions for future directions.

CMEs are the most recently (1971) discovered phenomena compared to other disturbances. The discovery of solar wind and interplanetary shocks preceded the 
CME discovery by a decade, while the discovery of SEPs was even earlier (1940s). Observationally, our understanding of CMEs has progressed significantly over the next four decades: a well-defined magnetic structure ejected along with the coronal plasma. While the vast majority of CMEs near the Sun do not drive a shock, the small number of shock-driving CMEs have the most intense consequences in the heliosphere. Although we treated SEP events as a transient, they are closely related to CMEs via their shocks. SEPs not related to shocks are generally the weak and short-lived impulsive events thought to be accelerated in the flare reconnection region. Once SEPs are released from the shock or flare site, their further propagation and evolution depends on the wave/plasma/magnetic properties of the background solar wind through which they propagate before reaching the observer. Shocks are also readily inferred from type II radio bursts, providing information on shocks from their origin close to the surface to $1 \mathrm{AU}$ and beyond. Interplanetary scintillation is another technique that can be used to track the turbulent sheath region of interplanetary shocks. SIRs/CIRs also drive shocks and accelerate particles, but generally at large distances from the Sun. Only about a fifth of IP shocks detected at Earth are due to CIRs. Particle acceleration in CIRs is significant beyond $1 \mathrm{AU}$. Geospace phenomena such as energetic storm particle events, ultra relativistic electron events, storm sudden commencement, and the onset of geomagnetically induced currents. The magnetic structure of the shock sheath and the driving CME determine the onset of a geomagnetic storm following shock arrival. The primary requirement for a storm is the presence of intense and prolonged southward pointing magnetic field in the sheath and/or the CME. This is the motivation behind the attempts to assess the internal magnetic field of CMEs when they are still near the Sun. For example, if the magnetic structure of the CME can be determined near the Sun, it should be possible to predict the structure in the heliosphere taking into account the environmental conditions. Both CIRs and shock sheaths are compressed heliospheric plasmas and hence have a similar impact on the magnetosphere in causing geomagnetic storms.

A wealth of observational information on CMEs and SIRs has accumulated over the past two decades, thanks to the fleet of space missions observing the Sun-Earth system. These data have contributed greatly to the current understanding of CMEs and SIRs. SOHO has provided extensive data over two solar cycles (23 and $24)$. In cycle 24, STEREO has provided multiview observations, enabling the determination of three-dimensional morphology of CMEs. The extended STEREO field of view allowed CMEs to be tracked from the coronal base to beyond Earth orbit, significantly enhancing observational knowledge of CME propagation. SOHO observations have shown that a magnetograph to measure the photospheric magnetic field, EUV imager to observe eruption signatures, white-light coronagraph to image CMEs, heliospheric imager for tracking CMEs to $1 \mathrm{AU}$, and a low-frequency radio telescope to detect shock signatures from close to the Sun to 1 AU. In addition to these, we also have instruments for in situ measurements of plasma, magnetic field, and energetic particles to complete the data set needed for investigating earth-affecting solar transients. The STEREO mission's twin spacecraft transited through the Sun-Earth Lagrange points L4 and L5 and demonstrated that these are ideal locations for placing these instruments to better observe Earth-directed CMEs. STEREO did not have a magnetograph to observe the photospheric magnetic field; $\mathrm{SOHO}$ did not have a magnetometer for detect CMEs and CIRs in the solar wind. Multiview magnetograms are important not only to track potentially eruptive active regions from behind the east limb before they rotate onto the disk but also to build global magnetic field distribution used as input to background solar wind models. While both L4 and L5 observations help characterize CMEs near the Sun, L5 vantage is useful in identifying active regions before they rotate into Earth view. On the other hand, L4 can observe Earth-directed CMEs without being affected by a "snowstorm" of secondary particles created by SEPs hitting the spacecraft. Earth-directed energetic CMEs are magnetically wellconnected to L5, so coronagraph images are vulnerable to such "snowstorms." Another advantage of the L5 location is that CIRs arrive at L5 a few days before they arrive at Earth, so one can predict the nature of CIRs arriving at Earth. Placing similar instruments at L4 and L5 will be ideal for a better characterization of CMEs near the Sun. Ideally, one should have multiple spacecraft at various locations in Earth orbit to provide space weather-relevant information on transients as well as the global magnetic field. Future efforts should also be directed toward using other techniques such as Faraday rotation to measure the magnetic content of CME flux ropes.

There has also been a rapid development of several MHD models with sophisticated simulation techniques to describe most or all stages of CMEs, i.e., pre-eruptive stage, destabilization and eruption, and propagation. In particular, models involving flux rope are growing in number so that the currently used hydrodynamic pulse representing a CME can be eventually replaced by a flux rope, which is more realistic and consistent with in situ observations. Such a transition would have the potential to predict the magnetic field vectors in the heliosphere rather than predicting just the CME arrival time. MHD models have also started considering the simulation 
boundaries closer to the Sun to account for forces that significantly affect the propagation of CMEs. The ultimate goal is to predict the magnetic field vectors at any point in the inner heliosphere, soon after the eruption at the Sun. Recent work on deriving the magnetic properties of CMEs near the Sun using source properties and eruption data will help test and improve global MHD modeling of CME propagation. However, we still have a long way to go in understanding when a magnetic region on the Sun hosts an eruption, but significant progress over the recent years in both terms of our observational and modeling capabilities is seamlessly contributing towards an eventual resolution of this cornerstone challenge. We still do not have a reliable set of active region parameters that would indicate an eruption. This is a common problem to both flares and CMEs because they are manifestations of a common energy release in the source magnetic region. Observations of the magnetic field at several layers above the photosphere and all around the Sun tied with advances in our capability to model magnetic fields from emergence to eruption are lending reasonable optimism.

The VarSITI program was launched at the peak phase of solar cycle 24, which turned out to be only half as strong as cycle 23. VarSITI investigations dealt with Earth-affecting solar transients in the background of the diminished solar activity and the related changes in the heliosphere into which solar disturbances propagated. The space weather consequences of CMEs and CIRs have proven to be mild in solar cycle 24 . This is the combined effect of the diminished number of energetic CMEs and the weakened heliospheric state. This was the smallest cycle in the space age, so we are able to expand our knowledge of extended parameter space of Earthaffecting phenomena. Current predictions of the strength of solar cycle 25 point to a weak cycle as well, and one can expect another cycle with mild space weather. The extended and uniform data set from $\mathrm{SOHO}$ have helped us characterize the solar cycles from the point of view of CME evolution and particle acceleration. The Parker Solar Probe and the Solar Orbiter are sampling the weak heliosphere and we expect to learn a lot on the behavior of Earth-affecting transients in the heliosphere.

\footnotetext{
Abbreviations

ACE: Advance Composition Explorer; ADAPT: Air Force Data Assimilate Photospheric Flux Transport; AWSoM: Alfvén wave solar model; CAWS ES: Climate And Weather of the Sun-Earth System; CME: Coronal mass ejection; CIR: Corotating interaction region; CORHEL: Coronal-heliosphere; $\mathrm{CH}$ : Coronal hole; EVE: EUV Variability Experiment; DSA: Diffusive shock acceleration; EEGGL: Eruptive Event Generator Gibson-Low; EUHFORIA: European Heliospheric Forecasting Information Asset; ESP: Energetic storm particle; FIP: First ionization potential; ICME: Interplanetary coronal mass ejection; IP: Interplanetary; LASCO: Large Angle and Spectrometric Coronagraph Experiment; LAT: Large area telescope; LDGRF: Long-duration gamma-ray flare; PFSS: Potential field
}

source surface; PSP: Parker Solar Probe; ISEST: International Study of Earthaffecting Solar Transients; LFM: Lyon-Fedder-Mobarry;

MAS: Magnetohydrodynamic Algorithm Outside a Sphere; MESSENGE R: Mercury Surface, Space Environment, Geochemistry and Ranging; MHD: Magnetohydrodnamics; PCA: Polar cap absorption; SCOSTEP: Scientific Committee on Solar-Terrestrial Physics; SDO: Solar Dynamic Observatory; SECCHI: Sun Earth Connection Coronal and Heliospheric Investigation; SEP: Solar energetic particle; SIR: Stream interaction region; SO: Solar Orbiter; SOHO: Solar and Heliospheric Observatory; SPR: Solar particle release; SSN: Sunspot number; STEREO: Solar Terrestrial Relations Observatory; STER EO/A: STEREO Ahead; STEREO/B: STEREO Behind; SWMF: Space Weather Modeling Framework; VarSITI: Variability of the Sun and Its Terrestrial Impact

\section{Acknowledgements}

$J Z, N G, S F$, and MD gratefully acknowledge the travel grant from Nagoya University to attend the workshop held in Nagoya, Japan, in November 2019, for initiating this review work. JZ thanks Prof. Kazuo Shiokawa for his assistance in guiding the process of this review work. SP thanks Dr. M. K. Georgoulis for useful discussions.

\section{Authors' contributions}

JZ provided the overall management of writing this review paper, which was a group effort of 19 individual authors. JZ, NG, and MT conceived the structure of the paper. JZ (leading author) contributed to S1 (Section 1). JZ (leading author), NG, and KD contributed to S2. BV (leading author), YW (leading author), BZ, and MD contributed to S3. FS (leading author), NL, and XF contributed to S4. DW (leading author) and NVN (leading author) contributed to S5. OM (leading author), NG, MID, ND, and ML contributed to S6. MT (leading author), KD, MD, and SGH contributed to S7. SP (leading author), MD, and JZ contributed to S8. MT (leading author), MD, and KD contributed to S9. NG (leading author) contributed to S10. The author(s) read and approved the final manuscript.

\section{Funding}

The ISEST project, which results in this review, is supported by the SCOSTEP/ VARSITI program. JZ was funded by NASA grants NNH17ZDA001N-HSWO2R, 80NSSC20K1274, and 80NSSC19K0082. SF is supported by the Strategic Priority Research Program of the Chinese Academy of Sciences Grant No. XDB 41000000, the National Natural Science Foundation of China (41774184 and 41974202), and the Specialized Research Fund for State Key Laboratories. NVN's work was supported by NASA contract NNX17AB73G. MID acknowledges the support of NASA grants 80NSSC18K0520, 80NSSC19K0079, 80NSSC20K1255, and 80NSSC18K1366. NL was supported by the NASA grant $80 \mathrm{NSSC} 20 \mathrm{K0700}$. XF is supported by the Natural Science Foundation of China under grants 41531073,41861164026, 42030204 .

\section{Availability of data and materials}

Data sharing not applicable to this article as no datasets were generated or analyzed during the current study.

\section{Declarations}

\section{Competing interests}

The authors declare that they have no competing interests.

\section{Author details}

'Department of Physics and Astronomy, George Mason University, 4400 University Dr., MSN 3F3, Fairfax, VA 22030, USA. ${ }^{2}$ Institute of Physics, University of Graz, Graz, Austria. ${ }^{3}$ Goddard Space Flight Center, Greenbelt, MD, USA. ${ }^{4}$ National Observatory of Athens, Institute for Astronomy, Astrophysics, Space Applications and Remote Sensing, Penteli, Athens, Greece. ${ }^{5}$ Lockheed Martin Solar and Astrophysics Laboratory, Palo Alto, CA, USA. ${ }^{6}$ Department of Physics, University of loannina, 45110 loannina, Greece. ${ }^{7}$ SIGMA Weather Group, State Key Laboratory of Space Weather, National Space Science Center, Chinese Academy of Sciences, Beijing 100190, China. ${ }^{8} \mathrm{Hvar}$ Observatory, Faculty of Geodesy, University of Zagreb, Kaciceva 26, HR-10000 Zagreb, Croatia. ${ }^{9}$ CAS Key Laboratory of Geospace Environment, Department of Geophysics and Planetary Sciences, University of Science and Technology of China, Hefei, Anhui 230026, PR China. ${ }^{10}$ ISR, Boston College, 140 Commonwealth Ave., Chestnut Hill, MA 02467, USA. ${ }^{11}$ Southwest Research Institute, 6220 Culebra Road, San Antonia, TX 78023, USA. 
${ }^{12}$ Department of Physics and Astronomy, University of Texas at San Antonio, San Antonio, TX 78249, USA. ${ }^{13}$ NorthWest Research Association, Boulder, CO, USA. ${ }^{14}$ Institut fuer Experimentelle und Angewandte Physik, University of Kiel, Kiel, Germany. ${ }^{15}$ Department of Physics and Astronomy, University of Turku, Turku, Finland. ${ }^{16}$ Max-Planck-Institut für Sonnensystemforschung, Justus-von-Liebig-Weg 3, 37077 Göttingen, Germany. ${ }^{17}$ INAF-Istituto di Astrofisica e Planetologia Spaziali, Via del Fosso del Cavaliere, 100, I-00133 Rome, Italy. ${ }^{18}$ Space Science Center and Department of Physics, University of New Hampshire, Durham, NH, USA.

\section{Received: 19 November 2020 Accepted: 26 April 2021} Published online: 04 October 2021

\section{References}

Abramenko V, Yurchyshyn V, Watanabe H (2009) Parameters of the magnetic flux inside coronal holes. Sol Phys 260(1):43-57. https://doi.org/10.1007/s11207009-9433-7

Afanasiev A, Vainio R, Rouillard AP, Battarbee M, Aran A, Zucca P (2018) Modelling of proton acceleration in application to a ground level enhancement. Astron Astrophys 614:A4

Agueda N, Lario D (2016) Release History and transport parameters of relativistic solar electrons inferred from near-the-sun in situ observations. Astrophys J 829:131. https://doi.org/10.3847/0004-637X/829/2/131

Agueda N, Vainio R, Lario D, Sanahuja B (2008) Injection and interplanetary transport of near-relativistic electrons: modeling the impulsive event on 2000 May 1. Astrophys J 675(2):1601. https://doi.org/10.1086/527527

Ajello M, Albert A, Allafort A, Baldini L, Guido B, Bastieri D, Bellazzini R et al (2014) Impulsive and long duration high-energy gamma-ray emission from the very bright 2012 March 7 solar flares. Astrophys J 789(1):20

Al-Haddad N, Nieves-Chinchilla T, Savani NP, Möstl C, Marubashi K, Hidalgo MA, Roussev II, Poedts S, Farrugia CJ (2013) Magnetic field configuration models and reconstruction methods for interplanetary coronal mass ejections. Sol Phys 284(1):129-149. https://doi.org/10.1007/s11207-013-0244-5

Al-Haddad N, Poedts S, Roussev I, Farrugia CJ, Yu W, Lugaz N (2019) The magnetic morphology of magnetic clouds: multi-spacecraft investigation of twisted and writhed coronal mass ejections. Astrophys J 870(2):100. https:// doi.org/10.3847/1538-4357/aaf38d

Al-Haddad N, Roussev II, Möstl C, Jacobs C, Lugaz N, Poedts S, Farrugia CJ (2011) On the internal structure of the magnetic field in magnetic clouds and interplanetary coronal mass ejections: writhe versus twist. Astrophys J 738(2): L18. https://doi.org/10.1088/2041-8205/738/2/L18

Allegrini F, Desai Ml, Mason GM, Kucharek H, Möbius E (2008) Evidence for massper-charge-dependent acceleration of a multiple-component seed population by CME-driven interplanetary shocks near 1 AU. Astrophys J 682(1):690

Altschuler MD, Newkirk G (1969) Magnetic fields and the structure of the solar corona. I: Methods of calculating coronal fields. Sol Phys 9:131-149. https:// doi.org/10.1007/BF00145734

Alzate N, Morgan H (2017) Identification of low coronal sources of 'stealth' coronal mass ejections using new image processing techniques. Astrophys J 840:103. https://doi.org/10.3847/1538-4357/aa6caa

Amerstorfer T, Möstl C, Hess P, Temmer M, Mays ML, Reiss MA, Lowrance P, Bourdin P-A (2018) Ensemble prediction of a halo coronal mass ejection using heliospheric imagers. Space Weather 16:784-801. https://doi.org/10.102 9/2017SW001786

An J, Magara T, Hayashi K, Moon Y-J (2019) Parametric study of ICME properties related to space weather disturbances via a series of three-dimensional MHD simulations. Sol Phys 294:143. https://doi.org/10.1007/s11207-019-1531-6

Anastasiadis A, Papaioannou A, Sandberg I, Georgoulis M, Tziotziou K, Kouloumvakos A, Jiggens P (2017) Predicting Flares and Solar Energetic Particle Events: The FORSPEF Tool. Sol Physics 292:134. https://doi.org/10.1 007/s11207-017-1163-7

Andrews MD (2003) A Search for CMEs Associated with Big Flares. Sol Physics 218:261-279. https://doi.org/10.1023/B:SOLA.0000013039.69550.bf

Antiochos SK (2013) Helicity condensation as the origin of coronal and solar wind structure. Astrophys J 772:72. https://doi.org/10.1088/0004-637X/772/1/ 72

Antiochos SK, DeVore CR, Klimchuk JA (1999) A model for solar coronal mass ejections. Astrophys J 510:485-493. https://doi.org/10.1086/306563

Aparna V, Martens PC (2020) Solar filaments and interplanetary magnetic field Bz. Astrophys J 897:68. https://doi.org/10.3847/1538-4357/ab908b
Arge CN, Pizzo VJ (2000) Improvement in the prediction of solar wind conditions using near-real time solar magnetic field updates. J Geophys Res 105:1046510480. https://doi.org/10.1029/1999JA000262

Aschwanden MJ (2012) GeV Particle acceleration in solar flares and ground level enhancement (GLE) events. Space Sci Rev 171:3-21

Aschwanden MJ, Nitta NV, Wuelser J-P, Lemen JR, Sandman A, Vourlidas A, Colaninno RC (2009) First measurements of the mass of coronal mass ejections from the EUV dimming observed with STEREO EUVI A+B spacecraft. Astrophys J 706:376-392. https://doi.org/10.1088/0004-637X/706/1/376

Asvestari E, Heinemann SG, Temmer M, Pomoell J, Kilpua E, Magdalenic J, Poedts S (2019) Reconstructing coronal hole areas with EUHFORIA and adapted WSA model: optimizing the model parameters. I Geophys Res (Space Physics) 124:8280-8297. https://doi.org/10.1029/2019JA027173

Attrill G, Nakwacki MS, Harra LK, van Driel-Gesztelyi L, Mandrini CH, Dasso S, Wang J (2006) Using the Evolution of coronal dimming regions to probe the global magnetic field topology. Sol Phys 238:117-139. https://doi.org/10.1 007/s11207-006-0167-5

Aulanier G (2013) The physical mechanisms that initiate and drive solar eruptions. Proc Int Astronomical Union 8(S300):184-196. https://doi.org/10.1017/S1743 921313010958

Aulanier G, DeLuca EE, Antiochos SK, McMullen RA, Golub L (2000) The topology and evolution of the Bastille Day Flare. Astrophys J 540:1126-1142. https:// doi.org/10.1086/309376

Aulanier G, Janvier M, Schmieder B (2012) The standard flare model in three dimensions. I. Strong-to-weak shear transition in post-flare loops. Astron Astrophys 543:A110. https://doi.org/10.1051/0004-6361/201219311

Aulanier G, Török T, Démoulin P, DeLuca EE (2010) Formation of torus-unstable flux ropes and electric currents in erupting sigmoids. Astrophys J 708:314333. https://doi.org/10.1088/0004-637X/708/1/314

Austin HJ, Savani NP (2018) Skills for forecasting space weather: skills for forecasting space weather. Weather 73(11):362-366. https://doi.org/10.1002/ wea.3076

Bain HM, Mays ML, Luhmann JG, Li Y, Jian LK, Odstrcil D (2016) Shock connectivity in the 2010 August and 2012 July solar energetic particle events inferred from observations and ENLIL modeling. Astrophys J 825(1):1. https:// doi.org/10.3847/0004-637X/825/1/1

Baker DN, Erickson PJ, Fennell JF, Foster JC, Jaynes AN, Verronen PT (2018) Space Weather Effects Earths Radiation Belts. 214(1):17. https://doi.org/10.1007/ s11214-017-0452-7

Baker DN, Li X, Pulkkinen A, Ngwira CM, Mays ML, Galvin AB, Simunac KDC (2013) A major solar eruptive event in July 2012: Defining extreme space weather scenarios. Space Weather 11(10):585-591. https://doi.org/10.1002/swe.20097

Bale SD, Badman ST, Wygant JR (2019) Highly structured slow solar wind emerging from an equatorial coronal hole. Nat 576:237-242. https://doi.org/1 $0.1038 / 541586-019-1818-7$

Balmaceda LA, Vourlidas A, Stenborg G, Cyr OCS (2020) On the expansion speed of coronal mass ejections: implications for self-similar evolution. Sol Phys 295: 107. https://doi.org/10.1007/s11207-020-01672-6

Balmaceda LA, Vourlidas A, Stenborg G, Lago AD (2018) How reliable are the properties of coronal mass ejections measured from a single viewpoint? Astrophys J 863:57. https://doi.org/10.3847/1538-4357/aacff8

Barczynski K, Aulanier G, Janvier M, Schmieder B, Masson S (2020) Electric current evolution at the footpoints of solar eruptions. Astrophys J 895:18. https://doi. org/10.3847/1538-4357/ab893d

Barczynski K, Aulanier G, Masson S, Wheatland MS (2019) Flare reconnectiondriven magnetic field and Lorentz force variations at the Sun's surface. Astrophys J 877:67. https://doi.org/10.3847/1538-4357/ab1b3d

Barnes D, Davies JA, Harrison RA, Byrne JP, Perry CH, Bothmer V, Eastwood JP et al (2019) CMEs in the heliosphere: II. A Statistical analysis of the kinematic properties derived from single-spacecraft geometrical modelling techniques applied to CMEs detected in the heliosphere from 2007 to 2017 by STEREO/ HI-1. Sol Phys 294:57. https://doi.org/10.1007/s11207-019-1444-4

Barnes, D., J. A. Davies, R. A. Harrison, J. P. Byrne, C. H. Perry, V. Bothmer, J. P. Eastwood, et al. (2020) "CMEs in the Heliosphere: III. A Statistical analysis of the kinematic properties derived from stereoscopic geometrical modelling techniques applied to CMEs detected in the heliosphere from 2008 to 2014 by STEREO/HI-1." ArXiv:2006.14879 [Astro-Ph, Physics:Physics], June. http://a rxiv.org/abs/2006.14879.

Barnes G (2007) On the relationship between coronal magnetic null points and solar eruptive events. Astrophys J 670(1):L53-L56. https://doi.org/10.1086/ 524107 
Bastian TS, Pick M, Kerdraon A, Maia D, Vourlidas A (2001) The coronal mass ejection of 1998 April 20: direct imaging at radio wavelengths. Astrophys J 558(1):L65-L69. https://doi.org/10.1086/323421

Baumgartner C, Thalmann JK, Veronig AM (2018) On the factors determining the eruptive character of solar flares. Astrophys J 853:105. https://doi.org/10.384 7/1538-4357/aaa243

Bein BM, Berkebile-Stoiser S, Veronig AM, Temmer M, Muhr N, Kienreich I, Utz D Vršnak B (2011) Impulsive acceleration of coronal mass ejections. I. Statistics and coronal mass ejection source region characteristics. Astrophys J 738:191. https://doi.org/10.1088/0004-637X/738/2/191

Bein BM, Berkebile-Stoiser S, Veronig AM, Temmer M, Vršnak B (2012) Impulsive acceleration of coronal mass ejections. II. Relation to soft $\mathrm{x}$-ray flares and filament eruptions. Astrophys J 755(1):44. https://doi.org/10.1088/0004-637X/ 755/1/44

Bell AR (1978) The Acceleration of Cosmic Rays in Shock Fronts - I. Mon Notices R Astronomical Soc 182(2):147-156. https://doi.org/10.1093/mnras/182.2.147

Bemporad A, Mancuso S (2010) First complete determination of plasma physical parameters across a coronal mass ejection-driven shock. Astrophys J 720(1): 130-143. https://doi.org/10.1088/0004-637X/720/1/130

Bemporad A, Zuccarello FP, Jacobs C, Mierla M, Poedts S (2012) Study of multiple coronal mass ejections at solar minimum conditions. Sol Phys. https://doi. org/10.1007/s11207-012-9999-3

Berger MA, Field GB (1984) The topological properties of magnetic helicity. J Fluid Mech 147:133-148.\&nbsp. https://doi.org/10.1017/S0022112084002019

Biesecker DA, Myers DC, Thompson BJ, Hammer DM, Vourlidas A (2002) Solar phenomena associated with "EIT Waves". Astrophys J 569:1009-1015. https:// doi.org/10.1086/339402

Bobra MG, Couvidat S (2015) Solar flare prediction using SDO/HMI vector magnetic field data with a machine-learning algorithm. Astrophys J 798(2): 135. https://doi.org/10.1088/0004-637X/798/2/135

Bobra MG, llonidis S (2016) Predicting coronal mass ejections using machine learning methods. Astrophys J 821(2):127. https://doi.org/10.3847/0004-637X/ $821 / 2 / 127$

Bocchialini K, Grison B, Menvielle M, Chambodut A, Cornilleau-Wehrlin N, Fontaine D, Marchaudon A et al (2018) Statistical analysis of solar events associated with storm sudden commencements over one year of solar maximum during cycle 23: propagation from the Sun to the Earth and effects. Sol Phys 293:75. https://doi.org/10.1007/s11207-018-1278-5

Bochsler P (2008) Composition of matter in the heliosphere. Proc Int Astronomical Union 4(S257):17-28

Borovsky JE, Denton MH (2006) Differences between CME-driven storms and CIRdriven storms. J Geophys Res (Space Physics) 111:A07S08. https://doi.org/10.1 029/2005JA011447

Bosman E, Bothmer V, Nisticò G, Vourlidas A, Howard RA, Davies JA (2012) Threedimensional properties of coronal mass ejections from STEREO/SECCH observations. Sol Phys 281:167-185. https://doi.org/10.1007/s11207-012-0123-5

Bothmer V, Schwenn R (1998) The structure and origin of magnetic clouds in the solar wind. Ann Geophysicae 16:1-24. https://doi.org/10.1007/s00585-9970001-x

Boucheron LE, Valluri M, McAteer RTJ (2016) Segmentation of coronal holes using active contours without edges. Sol Phys 291:2353-2372. https://doi.org/10.1 007/s11207-016-0985-Z

Boursier Y, Llebaria A, Goudail F, Lamy P, Robelus S (2005) Automatic detection of coronal mass ejections on LASCO-C2 synoptic maps. 5901:13-24. https:// doi.org/10.1117/12.616011

Burkepile JT, Hundhausen AJ, Stanger AL, Cyr OCS, Seiden JA (2004) Role of projection effects on solar coronal mass ejection properties: 1. A study of CMEs associated with limb activity. J Geophys Res Space Phys 109(A3). https://doi.org/10.1029/2003JA010149

Burlaga L, Berdichevsky D, Gopalswamy N, Lepping R, Zurbuchen T (2003) Merged interaction regions at 1 AU. J Geophys Res 108:1425. https://doi. org/10.1029/2003JA010088

Burlaga L, Sittler E, Mariani F, Schwenn R (1981) Magnetic loop behind an interplanetary shock - Voyager, Helios, and IMP 8 Observations. J Geophys Res 86:6673-6684. https://doi.org/10.1029/JA086iA08p06673

Burlaga LF (1988) Magnetic clouds and force-free fields with constant alpha. J Geophys Res 93(A7):7217. https://doi.org/10.1029/JA093iA07p07217

Byrne JP, Maloney SA, James McAteer RT, Refojo JM, Gallagher PT (2010) Propagation of an Earth-directed coronal mass ejection in three dimensions. Nat Commun 1:74. https://doi.org/10.1038/ncomms1077
Byrne JP, Morgan H, Habbal SR, Gallagher PT (2012) Automatic detection and tracking of coronal mass ejections. II. Multiscale filtering of coronagraph images. Astrophys J 752:145. https://doi.org/10.1088/0004-637X/752/2/145

Caffau E, Ludwig HG, Steffen M, Freytag B, Bonifacio P (2011) Solar Chemical Abundances Determined with a CO5BOLD 3D Model Atmosphere. 268(2): 255-269. https://doi.org/10.1007/s11207-010-9541-4

Camporeale $E$ (2019) The challenge of machine learning in space weather: nowcasting and forecasting. Space Weather 17(8):1166-1207. https://doi. org/10.1029/2018SW002061

Cane HV, McGuire REV, Von Rosenvinge TT (1986) Two classes of solar energetic particle events associated with impulsive and long-duration soft $\mathrm{x}$-ray flares. Astrophys J 301:448-459

Cane HV, Mewaldt RA, Cohen CMS, Von Rosenvinge TT (2006) Role of flares and shocks in determining solar energetic particle abundances. J Geophys Res Space Phys 111(A6)

Cane HV, Reames DV, Von Rosenvinge TT (1988) The role of interplanetary shocks in the longitude distribution of solar energetic particles. J Geophys Res Space Phys 93(A9):9555-9567

Cane HV, Richardson IG (2003) Interplanetary coronal mass ejections in the nearEarth solar wind during 1996-2002. J Geophys Res (Space Physics) 108:1156. https://doi.org/10.1029/2002JA009817

Canfield RC, Hudson HS, McKenzie DE (1999) Sigmoidal morphology and eruptive solar activity. Geophys Res Lett 26:627-630. https://doi.org/10.1029/1 999GL900105

Caplan RM, Downs C, Linker JA (2016) Synchronic coronal hole mapping using multi-instrument EUV images: data preparation and detection method. Astrophys J 823:53. https://doi.org/10.3847/0004-637X/823/1/53

Cargill PJ (2004) On the aerodynamic drag force acting on interplanetary coronal mass ejections. Sol Phys 221:135-149. https://doi.org/10.1023/B:SOLA. 0000033366.10725.a2

Carley EP, Vilmer N, Simões PJA, Fearraigh BÓ (2017) Estimation of a coronal mass ejection magnetic field strength using radio observations of gyrosynchrotron radiation. Astron Astrophys 608:A137. https://doi.org/10.1 051/0004-6361/201731368

Carrington RC (1859) Description of a singular appearance seen in the Sun on September 1, 1859. Mon Notices R Astronomical Soc 20:13-15. https://doi. org/10.1093/mnras/20.1.13

Chané E, Jacobs C, Van der Holst B, Poedts S, Kimpe D (2005) On the effect of the initial magnetic polarity and of the background wind on the evolution of CME shocks. Astron Astrophys 432(1):331-339. https://doi.org/10.1051/00046361:20042005

Chané E, Van der Holst B, Jacobs C, Poedts S, Kimpe D (2006) Inverse and normal coronal mass ejections: evolution up to 1 AU. Astron Astrophys 447(2):727733. https://doi.org/10.1051/0004-6361:20053802

Chen C, Liu YD, Wang R, Zhao X, Hu H, Zhu B (2019) Characteristics of a gradual filament eruption and subsequent $\mathrm{cme}$ propagation in relation to a strong geomagnetic storm. Astrophys J 884:90. https://doi.org/10.3847/1538-4357/a b3f36

Chen H, Zhang J, Ma S, Yang S, Li L, Huang X, Xiao J (2015) Confined flares in solar active region 12192 from 2014 October 18 to 29. Astrophys J Lett 808 : L24. https://doi.org/10.1088/2041-8205/808/1/L24

Chen J (1989) Effects of toroidal forces in current loops embedded in a background plasma. Astrophys J 338:453. https://doi.org/10.1086/167211

Chen J (2017) Physics of erupting solar flux ropes: coronal mass ejections (CMEs) - recent advances in theory and observation. Phys Plasmas 24:090501. https://doi.org/10.1063/1.4993929

Chen J, Cargill PJ, Palmadesso PJ (1997b) Predicting solar wind structures and their geoeffectiveness. J Geophys Res Space Phys 102(A7):14701-14720. https://doi.org/10.1029/97JA00936

Chen J, Howard RA, Brueckner GE, Santoro R, Krall J, Paswaters SE, Cyr OCS, Schwenn R, Lamy P, Simnett GM (1997a) Evidence of an erupting magnetic flux rope: LASCO coronal mass ejection of 1997 April 13. Astrophys J Lett 490:L191-L194. https://doi.org/10.1086/311029

Chen PF (2011) Coronal mass ejections: models and their observational basis. Living Rev Sol Phys 8. https://doi.org/10.12942//rsp-2011-1

Cheng X, Ding MD, Zhang J, Sun XD, Guo Y, Wang YM, Kliem B, Deng YY (2014) Formation of a double-decker magnetic flux rope in the sigmoidal solar active region 11520. Astrophys J 789:93. https://doi.org/10.1088/0004-637X/789/2/93

Cheng X, Yang G, Ding MD (2017) Origin and structures of solar eruptions I: Magnetic flux rope. Sci China Earth Sci 60:1383-1407. https://doi.org/10.1007/ s11430-017-9074-6 
Cheng X, Zhang J, Ding MD, Guo Y, Su JT (2011) A comparative study of confined and eruptive flares in NOAA AR 10720. Astrophys J 732:87. https:// doi.org/10.1088/0004-637X/732/2/87

Cheng X, Zhang J, Kliem B, Török T, Xing C, Zhou ZJ, Inhester B, Ding MD (2020) Initiation and early kinematic evolution of solar eruptions. Astrophys J 894(2): 85. https://doi.org/10.3847/1538-4357/ab886a

Cheng X, Zhang J, Olmedo O, Vourlidas A, Ding MD, Liu Y (2012) Investigation of the formation and separation of an extreme-ultraviolet wave from the expansion of a coronal mass ejection. Astrophys J Lett 745:L5. https://doi. org/10.1088/2041-8205/745/1/L5

Chi Y, Shen C, Luo B, Wang Y, Mengjiao X (2018a) Geoeffectiveness of stream interaction regions from 1995 to 2016. Space Weather 16:1960-1971. https:// doi.org/10.1029/2018SW001894

Chi Y, Shen C, Wang Y, Xu M, Ye P, Wang S (2016) Statistical study of the interplanetary coronal mass ejections from 1995 to 2015. Sol Phys 291(8): 2419-2439. https://doi.org/10.1007/s11207-016-0971-5

Chi Y, Zhang J, Shen C, Hess P, Liu L, Mishra W, Wang Y (2018b) Observational study of an Earth-affecting problematic ICME from STEREO. Astrophys J 863 108. https://doi.org/10.3847/1538-4357/aacf44

Chintzoglou G, Patsourakos S, Vourlidas A (2015) Formation of magnetic flux ropes during a confined flaring well before the onset of a pair of major coronal mass ejections. Astrophys J 809:34. https://doi.org/10.1088/0004-63 $7 X / 809 / 1 / 34$

Chmielewska E, Tomczak M, Kołomański S, Mrozek T (2016) Plasma diagnostics in two kinematic classes of CMEs observed by the atmospheric imaging assembly onboard the Solar Dynamic Observatory. Astronomische Nachr 337: 1016. https://doi.org/10.1002/asna.201612426

Cho K-S, Marubashi K, Kim R-S, Park S-H, Lim E-K, Kim S-J, Kumar P, Yurchyshyn V, Moon Y-J, Lee J-O (2017) Impact of the Icme-Earth geometry on the strength of the associated geomagnetic storm: the September 2014 and March 2015 events. J Korean Astronomical Soc 50:29-39. https://doi.org/10.5303/JKAS.201 7.50.2.29

Cho K-S, Park S-H, Marubashi K, Gopalswamy N, Akiyama S, Yashiro S, Kim R-S, Lim E-K (2013) Comparison of helicity signs in interplanetary CMEs and their solar source regions. Sol Phys 284:105-127. https://doi.org/10.1007/s11207013-0224-9

Cid C, Hidalgo MA, Nieves-Chinchilla T, Sequeiros J, Viñas AF (2002) Plasma and magnetic field inside magnetic clouds: a global study. Sol Phys 207(1):187198. https://doi.org/10.1023/A:1015542108356

Cliver EW (2000) Solar energetic particles: acceleration and transport. In: AIP Conference Proceedings, vol 516. American Institute of Physics, pp 103-119

Cliver EW (2016) Flare versus shock acceleration of high-energy protons in solar energetic particle events. Astrophys J 832(2):128

Cliver EW, Laurenza M, Storini M, Thompson BJ (2005) On the origins of solar EIT waves. Astrophys J 631:604-611. https://doi.org/10.1086/432250

Cohen CMS, Mason GM, Mewaldt RA (2017) Characteristics of solar energetic ions as a function of longitude. Astrophys J 843(2):132

Cohen CMS, Mewaldt RA, Cummings AC, Leske RA, Stone EC, Von Rosenvinge TT, Wiedenbeck ME (2003) Variability of spectra in large solar energetic particle events. Adv Space Res 32(12):2649-2654

Cohen CMS, Stone EC, Mewaldt RA, Leske RA, Cummings AC, Mason GM, Desai Ml, von Rosenvinge TT, Wiedenbeck ME (2005) Heavy ion abundances and spectra from the large solar energetic particle events of October-November 2003. J Geophys Res Space Phys 110(A9)

Colaninno RC, Vourlidas A (2015) Using multiple-viewpoint observations to determine the interaction of three coronal mass ejections observed on 2012 March 5. Astrophys J 815(1):70. https://doi.org/10.1088/0004-637X/815/1/70

Colaninno RC, Vourlidas A, Wu CC (2013) Quantitative comparison of methods for predicting the arrival of coronal mass ejections at earth based on multiview imaging. J Geophys Res (Space Physics) 118:6866-6879. https://doi. org/10.1002/2013JA019205

Corona-Romero P, Gonzalez-Esparza JA, Perez-Alanis CA, Aguilar-Rodriguez E, Dela-Luz V, Mejia-Ambriz JC (2017) Calculating travel times and arrival speeds of CMEs to Earth: an analytic tool for space weather forecasting. Space Weather 15:464-483. https://doi.org/10.1002/2016SW001489

Cranmer SR (2009) Coronal holes. Living Rev Sol Phys 6:3. https://doi.org/10.12 942/Irsp-2009-3

Cranmer SR, Gibson SE, Riley P (2017) Origins of the ambient solar wind: implications for space weather. SSr 212(3-4):1345-1384. https://doi.org/10.1 007/s11214-017-0416-y
Cranmer SR, Winebarger AR (2019) The properties of the solar corona and its connection to the solar wind. Araa 57:157-187. https://doi.org/10.1146/a nnurev-astro-091918-104416

Cremades H, Bothmer V (2004) On the three-dimensional configuration of coronal mass ejections. Astron Astrophys 422:307-322. https://doi.org/10.1 051/0004-6361:20035776

Cremades H, Bothmer V, Tripathi D (2006) Properties of structured coronal mass ejections in solar cycle 23. Adv Space Res 38(3):461-465. https://doi.org/10.1 016/j.asr.2005.01.095

Cremades H, Iglesias FA, Merenda LA (2020) Asymmetric expansion of coronal mass ejections in the low corona. Astron Astrophys 635:A100. https://doi. org/10.1051/0004-6361/201936664

D'Huys E, Seaton DB, De Groof A, Berghmans D, Poedts S (2017) Solar signatures and eruption mechanism of the August 14, 2010 coronal mass ejection (CME). J Space Weather Space Climate 7:A7. https://doi.org/10.1051/swsc/201 7006

D'Huys E, Seaton DB, Poedts S, Berghmans D (2014) Observational characteristics of coronal mass ejections without low-coronal signatures. Astrophys J 795:49. https://doi.org/10.1088/0004-637X/795/1/49

Dalla S, Marsh MS, Laitinen T (2015) Drift-induced deceleration of solar energetic particles. Astrophys J 808(1):62

Dasso S, Mandrini CH, Démoulin P, Luoni ML (2006) A new model-independent method to compute magnetic helicity in magnetic clouds. Astron Astrophys 455:349-359. https://doi.org/10.1051/0004-6361:20064806

Dasso S, Mandrini CH, Schmieder B, Cremades H, Cid C, Cerrato Y, Saiz E et al (2009) Linking two consecutive nonmerging magnetic clouds with their solar sources: tracking two consecutive magnetic clouds. J Geophys Res Space Phys 114(A2):n/a. https://doi.org/10.1029/2008JA013102

Davies JA, Harrison RA, Perry CH, Möstl C, Lugaz N, Rollett T, Davis CJ et al (2012) A self-similar expansion model for use in solar wind transient propagation studies. Astrophys J 750:23. https://doi.org/10.1088/0004-637X/750/1/23

Davies JA, Perry CH, Trines RMGM, Harrison RA, Lugaz N, Möstl C, Liu YD, Steed K (2013) Establishing a stereoscopic technique for determining the kinematic properties of solar wind transients based on a generalized self-similarly expanding circular geometry. Astrophys J 777:167. https://doi.org/10.1088/ 0004-637X/777/2/167

Dayeh MA, Desai MI, Dwyer JR, Rassoul HK, Mason GM, Mazur JE (2009) Composition and spectral properties of the 1 AU quiet-time suprathermal ion population during solar cycle 23. Astrophys J 693(2):1588

de Nolfo GA, Bruno A, Ryan JM, Dalla S, Giacalone J, Richardson IG, Christian ER et al (2019) Comparing long-duration gamma-ray flares and high-energy solar energetic particles. Astrophys J 879(2):90

Decker RB (1981) The modulation of low-energy proton distributions by propagating interplanetary shock waves: a numerical simulation. J Geophys Res Space Phys 86(A6):4537-4554

Démoulin P, Aulanier G (2010) Criteria for flux rope eruption: non-equilibrium versus torus instability. Astrophys J 718:1388-1399. https://doi.org/10.1088/ 0004-637X/718/2/1388

Démoulin P, Dasso S (2009) Causes and consequences of magnetic cloud expansion. Astron Astrophys 498(2):551-566. https://doi.org/10.1051/0004-63 $61 / 200810971$

Démoulin P, Dasso S, Janvier M (2013) Does spacecraft trajectory strongly affect detection of magnetic clouds? Astron Astrophys 550:A3. https://doi.org/10.1 051/0004-6361/201220535

Démoulin P, Mandrini CH, Van Driel-Gesztelyi L, Lopez Fuentes MC, Aulanier G (2002) The magnetic helicity injected by shearing motions. Sol Phys 207:87110. https://doi.org/10.1023/A:1015531804337

Denton MH, Borovsky JE (2017) The response of the inner magnetosphere to the trailing edges of high-speed solar-wind streams. J Geophys Res (Space Physics) 122:501-516. https://doi.org/10.1002/2016JA023592

Denton MH, Borovsky JE, Stepanova M, Valdivia JA (2016) Preface: Unsolved problems of magnetospheric physics. J Geophys Res (Space Physics) 121:10. https://doi.org/10.1002/2016JA023362

Desai M, Giacalone J (2016) Large gradual solar energetic particle events. Living Rev Sol Phys 13(1):3

Desai MI, Mason GM, Dayeh MA, Ebert RW, Mccomas DJ, Li G, Cohen CMS, Mewaldt RA, Schwadron NA, Smith CW (2016a) Spectral properties of large gradual solar energetic particle events. I. Fe, $\mathrm{O}$, and seed material. Astrophys J 816:68. https://doi.org/10.3847/0004-637X/816/2/68

Desai MI, Mason GM, Dayeh MA, Ebert RW, McComas DJ, Li G, Cohen CMS, Mewaldt RA, Schwadron NA, Smith CW (2016b) Spectral properties of large 
gradual solar energetic particle events. II. Systematic Q/M dependence of heavy ion spectral breaks. Astrophys J 828:106. https://doi.org/10.3847/0004$637 X / 828 / 2 / 106$

Desai MI, Mason GM, Dwyer JR, Mazur JE, Gold RE, Krimigis SM, Smith CW, Skoug RM (2003) Evidence for a suprathermal seed population of heavy ions accelerated by interplanetary shocks near 1 AU. Astrophys J 588(2):1149

Desai MI, Mason GM, Gold RE, Krimigis SM, Cohen CMS, Mewaldt RA, Mazur JE, Dwyer JR (2006) Heavy-ion elemental abundances in large solar energetic particle events and their implications for the seed population. Astrophys J 649(1):470

Desai MI, Mason GM, Wiedenbeck ME, Cohen CMS, Mazur JE, Dwyer JR, Gold RE et al (2004) Spectral properties of heavy ions associated with the passage of interplanetary shocks at 1 AU. Astrophys J 611(2):1156

Dewey RM, Baker DN, Anderson BJ, Benna M, Johnson CL, Korth H, Daniel J. Gershman, et al. (2015) Improving solar wind modeling at mercury: incorporating transient solar phenomena into the WSA-ENLIL model with the cone extension: improving solar wind modeling at Mercury. J Geophys Res Space Phys 120(7):5667-5685. https://doi.org/10.1002/2015JA021194

Dierckxsens M, Tziotziou K, Silvia D, Patsou I, Marsh MS, Crosby NB, Malandraki O, Tsiropoula G (2015) Relationship between Solar Energetic Particles and Properties of Flares and CMEs: Statistical Analysis of Solar Cycle 23 Events. Sol Phys 290(3):841-874

Dissauer K, Veronig AM, Temmer M, Podladchikova T (2019) Statistics of Corona Dimmings associated with coronal mass ejections. II. Relationship between coronal dimmings and their associated CMEs. Astrophys J 874:123. https:// doi.org/10.3847/1538-4357/ab0962

Dissauer K, Veronig AM, Temmer M, Podladchikova T, Vanninathan K (2018) Statistics of coronal dimmings associated with coronal mass ejections. I. Characteristic dimming properties and flare association. Astrophys J 863:169 https://doi.org/10.3847/1538-4357/aad3c6

Domingo V, Fleck B, Poland Al (1995) The SOHO mission: an overview. Sol Phys 162:1-37. https://doi.org/10.1007/BF00733425

Doran DJ, Dalla S, Zelina P (2019) Temporal evolution of heavy-ion spectra in solar energetic particle events. Sol Phys 294(5):55

Dresing N, Effenberger F, Gómez-Herrero R, Heber B, Klassen A, Kollhoff A, Richardson I, Theesen S (2020) Statistical results for solar energetic electron spectra observed over 12 Yr with STEREO/SEPT. Astrophys J 889(2):143

Dresing N, Gómez-Herrero R, Heber B, Hidalgo MA, Klassen A, Temmer M, Veronig A (2016) Injection of solar energetic particles into both loop legs of a magnetic cloud. Astron Astrophys 586:A55

Dresing N, Gómez-Herrero R, Heber B, Klassen A, Malandraki O, Dröge W, Kartavykh Y (2014) Statistical survey of widely spread out solar electron events observed with STEREO and ACE with special attention to anisotropies. Astron Astrophys 567:A27

Dresing N, Gómez-Herrero R, Heber B, Klassen A, Temmer M, Veronig A (2018) Long-lasting injection of solar energetic electrons into the heliosphere. Astron Astrophys 613:A21

Dresing N, Gómez-Herrero R, Klassen A, Heber B, Kartavykh Y, Dröge W (2012) The large longitudinal spread of solar energetic particles during the 17 January 2010 solar event. Solar Phy 281(1):281-300

Dröge W, Kartavykh YY, Dresing N, Heber B, Klassen A (2014) Wide longitudinal distribution of interplanetary electrons following the 7 February 2010 solar event: observations and transport modeling. J Geophys Res Space Phys 119(8):6074-6094

Dröge W, Kartavykh YY, Dresing N, Klassen A (2016) Multi-spacecraft observations and transport modeling of energetic electrons for a series of solar particle events in August 2010. Astrophys J 826(2):134

Dröge W, Kartavykh YY, Klecker B, Kovaltsov GA (2010) Anisotropic threedimensional focused transport of solar energetic particles in the inner heliosphere. Astrophys J 709(2):912-919. https://doi.org/10.1088/0004-637X/ 709/2/912

Duan A, Jiang C, He W, Feng X, Zou P, Cui J (2019) A study of pre-flare solar coronal magnetic fields: magnetic flux ropes. Astrophys J 884:73. https://doi. org/10.3847/1538-4357/ab3e33

Dumbović M, Čalogović J, Vršnak B, Manuela T, Leila Mays M, Veronig A, Piantschitsch I (2018a) The drag-based ensemble model (DBEM) for coronal mass ejection propagation. Astrophys J 854:180. https://doi.org/10.3847/153 8-4357/aaaa66

Dumbović M, Guo J, Temmer M, Mays ML, Veronig A, Heinemann SG, Dissauer K et al (2019) Unusual plasma and particle signatures at Mars and STEREO-A related to CME-CME interaction. Apj 880(1):18. https://doi.org/10.3847/153 8-4357/ab27ca

Dumbović M, Heber B, Vršnak B, Temmer M, Kirin A (2018b) An analytical diffusion-expansion model for forbush decreases caused by flux ropes. Astrophys J 860:71. https://doi.org/10.3847/1538-4357/aac2de

Eastwood JP, Biffis E, Hapgood MA, Green L, Bisi MM, Bentley RD, Wicks R, McKinnell LA, Gibbs M, Burnett C (2017) The economic impact of space weather: where do we stand? Risk Anal 37(2):206-218. https://doi.org/1 0.1111/risa.12765

Evans RM, Opher M, Oran R, van der Holst B, Sokolov IV, Frazin R, Gombosi TI, Vásquez A (2012) Coronal heating by surface alfvén wave damping: implementation in a global magnetohydrodynamics model of the solar wind. Astrophys J 756(2):155. https://doi.org/10.1088/0004-637X/756/2/155

Eyles CJ, Harrison RA, Davis CJ, Waltham NR, Shaughnessy BM, Mapson-Menard HCA, Bewsher D et al (2009) The heliospheric imagers onboard the STEREO mission. Sol Phys 254:387-445. https://doi.org/10.1007/s11207-008-9299-0

Falconer DA, Moore RL, Gary GA (2006) Magnetic causes of solar coronal mass ejections: dominance of the free magnetic energy over the magnetic twist alone. Astrophys J 644:1258-1272. https://doi.org/10.1086/503699

Falconer DA, Moore RL, Gary GA (2008) Magnetogram measures of total nonpotentiality for prediction of solar coronal mass ejections from active regions of any degree of magnetic complexity. Astrophys J 689:1433-1442. https://doi.org/10.1086/591045

Fan Y, Gibson SE (2003) The emergence of a twisted magnetic flux tube into a preexisting coronal arcade. Astrophys J 589(2):L105-L108. https://doi.org/10.1 086/375834

Fan Y, Gibson SE (2007) Onset of coronal mass ejections due to loss of confinement of coronal flux ropes. Astrophys J 668:1232-1245. https://doi. org/10.1086/521335

Farrugia CJ, Burlaga LF, Osherovich VA, Richardson IG, Freeman MP, Lepping RP, Lazarus AJ (1993) A study of an expanding interplanetary magnetic cloud and its interaction with the Earth's magnetosphere: the interplanetary aspect. J Geophys Res Space Phys 98(A5):7621-7632. https://doi.org/10.1029/92JA 02349

Farrugia CJ, Janoo LA, Torbert RB, Quinn JM, Ogilvie KW, Lepping RP, Fitzenreiter $R J$ et al (1999) A uniform-twist magnetic flux rope in the solar wind. In: AIP Conference Proceedings. AIP, Nantucket, pp 745-748. https://doi.org/10.1 $063 / 1.58724$

Feng X (2020) Magnetohydrodynamic modeling of the solar corona and heliosphere. Atmosphere, Earth, Ocean \& Space. Springer Singapore, Singapore. https://doi.org/10.1007/978-981-13-9081-4

Feng X, Ma X, Xiang C (2015) Data-Driven Modeling of the Solar wind from $1 R_{\text {s }}$ to $1 \mathrm{AU}$ : data-driven solar wind modeling. J Geophys Res Space Phys 120(12):10,159-10,174. https://doi.org/10.1002/2015JA021911

Feng X, Yang L, Changqing X, Wu ST, Zhou Y, Zhong DK (2010) Threedimensional solar wind modeling from the Sun to Earth by a SIP-CESE MHD model with a six-component grid. Astrophys J 723(1):300-319. https://doi. org/10.1088/0004-637X/723/1/300

Feng X, Yang L, Xiang C, Jiang C, Ma X, Wu ST, Zhong DK, Zhou Y (2012) Validation of the 3D AMR SIP-CESE solar wind model for four Carrington rotations. Sol Phys 279(1):207-229. https://doi.org/10.1007/s11207-012-9969-9

Fisher RR, Munro RH (1984) Coronal transient geometry. I - The flare-associated event of 1981 March 25. Astrophys J 280:428-439. https://doi.org/10.1086/1 62009

Fletcher L, Dennis BR, Hudson HS, Krucker S, Phillips K, Veronig A, Battaglia M et al (2011) An observational overview of solar flares. Space Sci Rev 159(1):19. https://doi.org/10.1007/s11214-010-9701-8

Fokker AD (1963) Type IV solar radio emission. Space Sci Rev 2(1):70-90. https:// doi.org/10.1007/BF00174028

Forbes TG (2000) A review on the genesis of coronal mass ejections. J Geophys Res Space Phys 105(A10):23153-23165. https://doi.org/10.1029/2000JA000005

Forbush SE (1946) Three unusual cosmic-ray increases possibly due to charged particles from the Sun. Phys Rev 70(9-10):771-772. https://doi.org/10.1103/ PhysRev.70.771

Fox NJ, Velli MC, Bale SD, Decker R, Driesman A, Howard RA, Kasper JC et al (2016) The Solar Probe Plus Mission: humanity's first visit to our star. Space Sci Rev 204:7-48. https://doi.org/10.1007/s11214-015-0211-6

Garton TM, Gallagher PT, Murray SA (2018) Automated coronal hole identification via multi-thermal intensity segmentation. J Space Weather Space Climate 8: A02. https://doi.org/10.1051/swsc/2017039 
Georgoulis MK, Nindos A, Zhang H (2019) The source and engine of coronal mass ejections. Philos Trans R Soc London Ser A 377:20180094. https://doi, org/10.1098/rsta.2018.0094

Georgoulis MK, Rust DM (2007) Quantitative forecasting of major solar flares. Astrophys J Lett 661:L109-L112. https://doi.org/10.1086/518718

Giacalone J, Jokipii JR (2012) The longitudinal transport of energetic ions from impulsive solar flares in interplanetary space. Astrophys J 751(2):L33. https://doi.org/10.1088/2041-8205/751/2/L33

Gibson SE (2018) Solar prominences: theory and models. fleshing out the magnetic skeleton. Living Rev Sol Phys 15:7. https://doi.org/10.1007/s41116018-0016-2

Gibson SE, Foster D, Burkepile J, de Toma G, Stanger A (2006) The calm before the storm: the link between quiescent cavities and coronal mass ejections. Astrophys J 641:590-605. https://doi.org/10.1086/500446

Gibson SE, Low BC (1998) A time-dependent three-dimensional magnetohydrodynamic model of the coronal mass ejection. Astrophys J 493(1):460-473. https://doi.org/10.1086/305107

Gloeckler G (2003) Ubiquitous suprathermal tails on the solar wind and pickup ion distributions. In: AIP Conference Proceedings, vol 679. American Institute of Physics, pp 583-588

Gold T (1962) Magnetic storms. Space Sci Rev 1(1):100-114. https://doi.org/10.1 007/BF00174637

Gold T, Hoyle F (1960) On the origin of solar flares. Mon Not R Astron Soc 120:89. https://doi.org/10.1093/mnras/120.2.89

Goldstein H (1983) On the field configuration in magnetic clouds. In: NASA Conference Publication https://ui.adsabs.harvard.edu/abs/1983NASCP.2280. $731 \mathrm{G} / \mathrm{abstract}$

Gombosi Tl, van der Holst B, Manchester WB, Sokolov IV (2018) Extended MHD modeling of the steady solar corona and the solar wind. Living Rev Sol Phys 15(1):4. https://doi.org/10.1007/s41116-018-0014-4

Gómez-Herrero R, Dresing N, Klassen A, Bernd H, Lario D, Agueda N, Malandraki OE, Blanco JJ, Rodríguez-Pacheco J, Banjac S (2015) Circumsolar Energetic particle distribution on 2011 November 3. Astrophys J 799(1):55

Gómez-Herrero R, Dresing N, Klassen A, Heber B, Temmer M, Veronig A, Bučík R et al (2017) Sunward-propagating solar energetic electrons inside multiple interplanetary flux ropes. Astrophys J 840(2):85

Gontikakis C, Kontogiannis I, Georgoulis MK, Guennou C, Syntelis P, Park SH, Buchlin E (2020) Differential emission measure evolution as a precursor of solar flares. ArXiv E-Prints 2011:arXiv:2011.06433 http://adsabs.harvard.edu/a bs/2020arXiv201106433G

Gonzalez WD, Tsurutani BT, Clúa de Gonzalez AL (1999) Interplanetary origin of geomagnetic storms. Space Sci Rev 88:529-562. https://doi.org/10.1023/A:1 005160129098

Gonzalez WD, Tsurutani BT, Gonzalez ALC, Smith EJ, Tang F, Akasofu S-I (1989) Solar wind-magnetosphere coupling during intense magnetic storms (1978-1979). J Geophys Res 94:8835-8851. https://doi.org/10.1029/JA094iA 07 p08835

Good SW, Kilpua EKJ, LaMoury AT, Forsyth RJ, Eastwood JP, Möstl C (2019) Selfsimilarity of ICME flux ropes: observations by radially aligned spacecraft in the inner heliosphere. J Geophys Res Space Phys 124(7):4960-4982. https:// doi.org/10.1029/2019JA026475

Gopalswamy N (2016) History and development of coronal mass ejections as a key player in solar terrestrial relationship. Geosci Lett 3(1):8. https://doi.org/1 0.1186/s40562-016-0039-2

Gopalswamy N, Akiyama S, Yashiro S (2020b) The state of the heliosphere revealed by limb-halo coronal mass ejections in solar cycles 23 and 24. Astrophys J Lett 897:L1. https://doi.org/10.3847/2041-8213/ab9b7b

Gopalswamy N, Akiyama S, Yashiro S, Michalek G, Lepping RP (2008) Solar sources and geospace consequences of interplanetary magnetic clouds observed during solar cycle 23. J Atmos Sol Terr Phys 70:245-253. https://doi. org/10.1016/j.jastp.2007.08.070

Gopalswamy N, Akiyama S, Yashiro S, Michalek G, Xie H, Mäkelä P (2000a) Effect of the weakened heliosphere in solar cycle 24 on the properties of coronal mass ejections. ArXiv E-Prints:arXiv:2007.08291 http://adsabs.harvard.edu/a bs/2020arXiv200708291G

Gopalswamy N, Akiyama S, Yashiro S, Xie H (2017c) A new technique to provide realistic input to CME forecasting models. Proc Int Astron Union 13(S335): 258-262. https://doi.org/10.1017/\$1743921317011048

Gopalswamy N, Akiyama S, Yashiro S, Xie H (2018a) Coronal flux ropes and their interplanetary counterparts. J Atmos Sol Terr Phys 180:35-45. https://doi. org/10.1016/j.jastp.2017.06.004
Gopalswamy N, Akiyama S, Yashiro S, Xie H (2018d) A new technique to provide realistic input to CME forecasting models. In: Foullon C, Malandraki OE (eds) Space Weather of the Heliosphere: Processes and Forecasts, vol 335. IAU Symposium, pp 258-262. https://doi.org/10.1017/S1743921317011048

Gopalswamy N, Akiyama S, Yashiro S, Xie H, Mäkelä P, Michalek G (2014a) Anomalous expansion of coronal mass ejections during solar cycle 24 and its space weather implications. Geophys Res Lett 41:2673-2680. https://doi.org/1 0.1002/2014GL059858

Gopalswamy N, Lara A, Lepping RP, Kaiser ML, Berdichevsky D, Cyr OCS (2000) Interplanetary acceleration of coronal mass ejections. Geophys Res Lett 27(2): 145-148. https://doi.org/10.1029/1999GL003639

Gopalswamy N, Lara A, Yashiro S, Kaiser ML, Howard RA (2001b) Predicting the 1AU arrival times of coronal mass ejections. J Geophys Res Space Phys 106(A12):29207-29217. https://doi.org/10.1029/2001JA000177

Gopalswamy N, Mäkelä P, Akiyama S, Yashiro S, Xie H, Thakur N (2018b) Sun-toEarth propagation of the 2015 June 21 coronal mass ejection revealed by optical, EUV, and radio observations. J Atmos Sol Terr Phys 179:225-238. https://doi.org/10.1016/j.jastp.2018.07.013

Gopalswamy N, Mäkelä P, Xie H, Akiyama S, Yashiro S (2009a) CME interactions with coronal holes and their interplanetary consequences. J Geophys Res 114:A00A22. https://doi.org/10.1029/2008JA013686

Gopalswamy N, Mäkelä P, Xie H, Yashiro S (2013a) Testing the empirical shock arrival model using quadrature observations. Space Weather 11:661-669. https://doi.org/10.1002/2013SW000945

Gopalswamy N, Mäkelä P, Yashiro S (2019) A catalog of type II radio bursts observed by Wind/WAVES and their statistical properties. Sun Geosphere 14: 111-121. https://doi.org/10.31401/SunGeo.2019.02.03

Gopalswamy N, Mäkelä P, Yashiro S, Lara A, Xie H, Akiyama S, MacDowall RJ (2018e) Interplanetary type II radio bursts from Wind/WAVES and sustained gamma-ray emission from Fermi/LAT: evidence for shock source. Astrophys J Lett 868:L19. https://doi.org/10.3847/2041-8213/aaef36

Gopalswamy N, Mäkelä P, Yashiro S, Thakur N, Akiyama S, Xie H (2017a) A hierarchical relationship between the fluence spectra and CME kinematics in large solar energetic particle events: a radio perspective. J Phys Conf Ser 900: $1-11$

Gopalswamy N, Shimojo M, Lu W, Yashiro S, Shibasaki K, Howard RA (2003) Prominence eruptions and coronal mass ejection: a statistical study using microwave observations. Astrophys J 586(1):562-578. https://doi.org/10.1 086/367614

Gopalswamy N, Thompson WT, Davila JM, Kaiser ML, Yashiro S, Mäkelä P, Michalek G, Bougeret J-L, Howard RA (2009b) Relation between type II bursts and CMEs inferred from STEREO observations. Sol Phys 259:227-254. https:// doi.org/10.1007/s11207-009-9382-1

Gopalswamy N, Tsurutani B, Yan Y (2015c) Short-term variability of the Sun-Earth system: an overview of progress made during the CAWSES-II period. Prog Earth Planetary Sci 2:13. https://doi.org/10.1186/s40645-015-0043-8

Gopalswamy N, Xie H, Akiyama S, Mäkelä P, Yashiro S, Michalek G (2015a) The peculiar behavior of halo coronal mass ejections in solar cycle 24 . Astrophys J Lett 804:L23. https://doi.org/10.1088/2041-8205/804/1/L23

Gopalswamy N, Xie H, Akiyama S, Mäkelä PA, Yashiro S (2014b) Major solar eruptions and high-energy particle events during solar cycle 24. Earth Planets Space 66:104. https://doi.org/10.1186/1880-5981-66-104

Gopalswamy N, Xie H, Mäkelä P, Yashiro S, Akiyama S, Uddin W, Srivastava AK et al (2013b) Height of shock formation in the solar corona inferred from observations of type $\|$ radio bursts and coronal mass ejections. Adv Space Res 51(11):1981-1989

Gopalswamy N, Yashiro S (2011) The strength and radial profile of the coronal magnetic field from the standoff distance of a coronal mass ejection-driven shock. Astrophys J Lett 736:L17. https://doi.org/10.1088/2041-8205/736/1/L17

Gopalswamy N, Yashiro S, Akiyama S (2007) Geoeffectiveness of halo coronal mass ejections. J Geophys Res (Space Physics) 112:A06112. https://doi.org/1 0.1029/2006JA012149

Gopalswamy N, Yashiro S, Akiyama S, Xie H (2017b) Estimation of reconnection flux using post-eruption arcades and its relevance to magnetic clouds at 1 AU. Sol Phys 292:65. https://doi.org/10.1007/s11207-017-1080-9

Gopalswamy N, Yashiro S, Kaiser ML, Howard RA, Bougeret J-L (2001a) Radio Signatures of coronal mass ejection interaction: coronal mass ejection cannibalism? Astrophys J 548(1):L91-L94. https://doi.org/10.1086/318939

Gopalswamy N, Yashiro S, Kaiser ML, Thompson BJ, Plunkett S (1999) Multiwavelength signatures of coronal mass ejection, pp 207-210 http://adsabs.ha rvard.edu/abs/1999spro.proc..207G 
Gopalswamy N, Yashiro S, Krucker S, Stenborg G, Howard RA (2004) Intensity variation of large solar energetic particle events associated with coronal mass ejections. J Geophys Res Space Phys 109(A12)

Gopalswamy N, Yashiro S, Mäkelä P, Xie H, Akiyama S, Monstein C (2018c) Extreme kinematics of the 2017 September 10 solar eruption and the spectral characteristics of the associated energetic particles. Astrophys I Lett 863:L39. https://doi.org/10.3847/2041-8213/aad86c

Gopalswamy N, Yashiro S, Thakur N, Mäkelä P, Xie H, Akiyama S (2016) The 2012 July 23 backside eruption: an extreme energetic particle event? Astrophys $J$ 833(2):216

Gopalswamy N, Yashiro S, Xie H, Akiyama S, Mäkelä P (2015b) Properties and geoeffectiveness of magnetic clouds during solar cycles 23 and 24. J Geophys Res (Space Physics) 120:9221-9245. https://doi.org/10.1002/2015JA 021446

Gosling JT (1990) Coronal mass ejections and magnetic flux ropes in interplanetary space. In: Russell CT, Priest ER, Lee LC (eds) Geophysical Monograph Series, vol 58. American Geophysical Union, Washington, D. C, pp 343-364. https://doi.org/10.1029/GM058p0343

Gosling JT (1993) The solar flare myth. J Geophys Res 98 http://adsabs.harvard. edu/abs/1993JGR....98.....G

Gosling JT (2012) Magnetic reconnection in the solar wind. Space Sci Rev 172(14):187-200. https://doi.org/10.1007/s11214-011-9747-2

Gosling JT, Borrini G, Asbridge JR, Bame SJ, Feldman WC, Hansen RT (1981) Coronal streamers in the solar wind at $1 \mathrm{AU}$. Jgr 86(A7):5438-5448. https:// doi.org/10.1029/JA086iA07p05438

Gosling JT, Pizzo VJ (1999) Formation and evolution of corotating interaction regions and their three dimensional structure. SSr 89:21-52. https://doi.org/1 0.1023/A:1005291711900

Gou T, Veronig AM, Liu R, Zhuang B, Dumbović M, Podladchikova T, Reid HAS et al (2020) Solar flare-CME coupling throughout two acceleration phases of a fast CME. Astrophys J Lett 897:L36. https://doi.org/10.3847/2041-8213/ab9ec5

Grandin M, Aikio AT, Kozlovsky A (2019) Properties and Geoeffectiveness of solar wind high-speed streams and stream interaction regions during solar cycles 23 and 24. J Geophys Res (Space Physics) 124:3871-3892. https://doi.org/10.1 029/2018JA026396

Grechnev W, Kiselev VI, Meshalkina NS, Chertok IM (2015) Relations between microwave bursts and near-Earth high-energy proton enhancements and their origin. Sol Phys 290(10):2827-2855

Green LM, Fuentes MCL, Mandrini CH, Démoulin P, Van Driel-Gesztelyi L, Culhane JL (2002) The magnetic helicity budget of a CME-prolific active region. Sol Phys 208:43-68. https://doi.org/10.1023/A:1019658520033

Green LM, Kliem B (2009) Flux rope formation preceding coronal mass ejection onset. Astrophys J Lett 700:L83-L87. https://doi.org/10.1088/0004-637X/ 700/2/L83

Green LM, Kliem B, Török T, van Driel-Gesztelyi L, Attrill GDR (2007) Transient coronal sigmoids and rotating erupting flux ropes. Sol Phys 246:365-391. https://doi.org/10.1007/s11207-007-9061-z

Green LM, Török T, Vršnak B, Manchester W, Veronig A (2018) The origin, early evolution and predictability of solar eruptions. Space Sci Rev 214(1):46. https://doi.org/10.1007/s11214-017-0462-5

Gressl C, Veronig AM, Temmer M, Odstrčil D, Linker JA, Mikić Z, Riley P (2014) Comparative study of MHD modeling of the background solar wind. Sol Phys 289:1783-1801. https://doi.org/10.1007/s11207-013-0421-6

Groth CPT, De Zeeuw DL, Gombosi Tl, Powell KG (2000) Global ThreeDimensional MHD Simulation of a space weather event: CME formation, interplanetary propagation, and interaction with the magnetosphere. J Geophys Res 105:25053-25078. https://doi.org/10.1029/2000JA900093

Guennou C, Pariat E, Leake JE, Vilmer N (2017) Testing predictors of eruptivity using parametric flux emergence simulations. I Space Weather Space Climate 7:A17. https://doi.org/10.1051/swsc/2017015

Gui B, Shen C, Wang Y, Ye P, Liu J, Wang S, Zhao X (2011) Quantitative analysis of CME deflections in the corona. Sol Phys 271(1-2):111-139. https://doi.org/1 $0.1007 /$ s11207-011-9791-9

Guo Y, Cheng X, Ding MD (2017) Origin and structures of solar eruptions II: Magnetic modeling. Sci China Earth Sci 60(8):1408-1439. https://doi.org/10.1 007/s11430-017-9081-x

Guo Y, Xia C, Rony K, Ding MD, Chen PF (2019) Solar magnetic flux rope eruption simulated by a data-driven magnetohydrodynamic model. Astrophys $\rfloor$ 870(2):L21. https://doi.org/10.3847/2041-8213/aafabf

Harra LK (2009) On-disk signatures of eruptive activity from the Hinode mission. Adv Space Res 44(4):446-450. https://doi.org/10.1016/j.asr.2009.04.020
Harra LK, Sterling AC (2001) Material outflows from coronal intensity "dimming regions" during coronal mass ejection onset. Astrophys J Lett 561:L215-L218. https://doi.org/10.1086/324767

Harrison RA (1995) The nature of solar flares associated with coronal mass ejection. Astron Astrophys 304:585 http://adsabs.harvard.edu/abs/1995A\%2 6A...304..585H

Harrison RA, Davies JA, Barnes D, Byrne JP, Perry CH, Bothmer V, Eastwood JP et al (2018) CMEs in the Heliosphere: I. A Statistical Analysis of the observational properties of CMEs detected in the heliosphere from 2007 to 2017 by STEREO/HI-1. Sol Phys 293:77. https://doi.org/10.1007/s11207-018-1297-2

Harrison RA, Davies JA, Möstl C, Liu Y, Temmer M, Bisi MM, Eastwood JP et al (2012) An analysis of the origin and propagation of the multiple coronal mass ejections of 2010 August 1. Astrophys J 750(1):45. https://doi.org/10.1 088/0004-637X/750/1/45

Harrison RA, Lyons M (2000) A spectroscopic study of coronal dimming associated with a coronal mass ejection. Astron Astrophys 358:1097-1108 http://adsabs.harvard.edu/abs/2000A\%26A...358.1097H

Harvey KL, Recely F (2002) Polar coronal holes during cycles 22 and 23. Sol Phys 211(1):31-52. https://doi.org/10.1023/A:1022469023581

Hassanin A, Kliem B (2016) Helical kink instability in a confined solar eruption. Astrophys J 832:106. https://doi.org/10.3847/0004-637X/832/2/106

Hassler DM, Dammasch IE, Lemaire P, Brekke P, Curdt W, Mason HE, Vial J-C, Wilhelm K (1999) Solar wind outflow and the chromospheric magnetic network. Science 283:810. https://doi.org/10.1126/science.283.5403.810

Hayashi K (2012) An MHD Simulation model of time-dependent co-rotating solar wind: MHD Simulation Of Solar Wind. J Geophys Res Space Phys 117(A8):n/a. https://doi.org/10.1029/2011JA017490

Hayashi K, Feng X, Xiong M, Jiang C (2018) An MHD simulation of solar active region 11158 driven with a time-dependent electric field determined from HMI vector magnetic field measurement data. Astrophys J 855(1):11. https:// doi.org/10.3847/1538-4357/aaacd8

Hayashi K, Feng X, Xiong M, Jiang C (2019) Magnetohydrodynamic simulations for solar active regions using time-series data of surface plasma flow and electric field inferred from helioseismic magnetic imager vector magnetic field measurements. Astrophys J Lett 871:L28. https://doi.org/10.3847/20418213/aaffcf

Hayashi K, Hoeksema JT, Liu Y, Bobra MG, Sun XD, Norton AA (2015) The Helioseismic and Magnetic Imager (HMI) vector magnetic field pipeline: magnetohydrodynamics simulation module for the global solar corona. Sol Phys 290(5):1507-1529. https://doi.org/10.1007/s11207-015-0686-z

He H-Q, Wan W (2015) Numerical study of the longitudinally asymmetric distribution of solar energetic particles in the heliosphere. Astrophys J Suppl Ser 218(2):17. https://doi.org/10.1088/0067-0049/218/2/17

He W, Liu YD, Hu H, Wang R, Zhao X (2018) A stealth CME bracketed between slow and fast wind producing unexpected geoeffectiveness. Astrophys J 860: 78. https://doi.org/10.3847/1538-4357/aac381

Heinemann SG, Hofmeister SJ, Veronig AM, Temmer M (2018b) Three-phase evolution of a coronal hole. II. The magnetic field. Astrophys J 863:29. https:// doi.org/10.3847/1538-4357/aad095

Heinemann SG, Jerčić V, Temmer M, Hofmeister SJ, Dumbović M, Vennerstrom S, Verbanac G, Veronig AM (2020) A statistical study of the long-term evolution of coronal hole properties as observed by SDO. Astron Astrophys 638:A68. https://doi.org/10.1051/0004-6361/202037613

Heinemann SG, Temmer M, Farrugia CJ, Dissauer K, Kay C, Wiegelmann T, Dumbović M et al (2019a) CME-HSS interaction and characteristics tracked from Sun to Earth. Sol Phys 294(9):121. https:/doi.org/10.1007/s11207-019-1515-6

Heinemann SG, Temmer M, Heinemann N, Dissauer K, Samara E, Jerčić V, Hofmeister SJ, Veronig AM (2019b) Statistical analysis and catalog of nonpolar coronal holes covering the SDO-Era using CATCH. Sol Phys 294(10):144. https://doi.org/10.1007/s11207-019-1539-y

Heinemann SG, Temmer M, Hofmeister SJ, Veronig AM, Vennerstrøm S (2018a) Three-phase evolution of a coronal hole. I. $360^{\circ}$ remote sensing and in situ observations. Apj 861(2):151. https://doi.org/10.3847/1538-4357/aac897

Hess P, Colaninno RC (2017) Comparing automatic CME detections in multiple LASCO and SECCHI catalogs. Astrophys J 836(1):134

Hess P, Rouillard AP, Kouloumvakos A, Liewer PC, Zhang J, Dhakal S, Stenborg G, Colaninno RC, Howard RA (2020) WISPR imaging of a pristine CME. Astrophys J Suppl Ser 246:25. https://doi.org/10.3847/1538-4365/ab4ffo

Hess P, Zhang J (2014) Stereoscopic study of the kinematic evolution of a coronal mass ejection and its driven shock from the Sun to the Earth and the Prediction of their arrival times. Astrophys J 792(1):49 
Hess P, Zhang J (2017) A Study of the Earth-affecting CMEs of solar cycle 24. Sol Phys 292(6):80

Hickmann KS, Godinez HC, Henney CJ, Nick Arge C (2015) Data assimilation in the ADAPT photospheric flux transport model. Sol Phys 290(4):1105-1118. https://doi.org/10.1007/s11207-015-0666-3

Hidalgo MA (2002) Elliptical Cross-section model for the magnetic topology of magnetic clouds. Geophys Res Lett 29(13):1637. https://doi.org/10.1029/2001 GL013875

Hidalgo MA (2003) A study of the expansion and distortion of the cross section of magnetic clouds in the interplanetary medium. J Geophys Res 108(A8): 1320. https://doi.org/10.1029/2002JA009818

Hidalgo MA (2011) A study of the electric field induced by magnetic clouds. J Geophys Res (Space Physics) 116(A2):A02101. https://doi.org/10.1029/2010JA 016048

Hidalgo MA (2013) A global magnetic topology model for magnetic clouds. II. Astrophys J 766(2):125. https://doi.org/10.1088/0004-637X/766/2/125

Hidalgo MA (2016) A global magnetic topology model for magnetic clouds. IV. Astrophys J 823(1):3. https://doi.org/10.3847/0004-637X/823/1/3

Hidalgo MA, Cid C, Medina J, Viñas AF (2000) A New model for the topology of magnetic clouds in the solar wind. Sol Phys 194(1):165-174. https://doi.org/1 0.1023/A:1005206107017

Hidalgo MA, Cid C, Vinas AF, Sequeiros J (2002) A non-force-free approach to the topology of magnetic clouds in the solar wind. J Geophys Res 107(A1):1002. https://doi.org/10.1029/2001JA900100

Hidalgo MA, Nieves-Chinchilla T (2012) A global magnetic topology model for magnetic clouds. I. Astrophys J 748(2):109. https://doi.org/10.1088/0004-63 $7 X / 748 / 2 / 109$

Hinterreiter J, Magdalenic J, Temmer M, Verbeke C, Jebaraj IC, Samara E, Asvestari E et al (2019) Assessing the performance of EUHFORIA modeling the background solar wind. Sol Phys 294:170. https://doi.org/10.1007/s11207-01 9-1558-8

Hodgson R (1859) On a curious appearance seen in the Sun. Mon Notices R Astron Soc 20:15-16. https://doi.org/10.1093/mnras/20.1.15

Hofmeister SJ, Utz D, Heinemann SG, Veronig A, Temmer M (2019) Photospheric magnetic structure of coronal holes. Aap 629:A22. https://doi.org/10.1051/ 0004-6361/201935918

Hofmeister SJ, Veronig A, Reiss MA, Temmer M, Vennerstrom S, Vršnak B, Heber B (2017) Characteristics of low-latitude coronal holes near the maximum of solar cycle 24. Apj 835(2):268. https://doi.org/10.3847/1538-4357/835/2/268

Hofmeister SJ, Veronig A, Temmer M, Vennerstrom S, Heber B, Vršnak B (2018) The dependence of the peak velocity of high-speed solar wind streams as measured in the ecliptic by ACE and the STEREO satellites on the area and co-latitude of their solar source coronal holes. J Geophys Res (Space Physics) 123(3):1738-1753. https://doi.org/10.1002/2017JA024586

Hood AW, Priest ER (1981) Critical conditions for magnetic instabilities in forcefree coronal loops. Geophys Astrophys Fluid Dyn 17(1):297-318. https://doi. org/10.1080/03091928108243687

Hori K, Culhane JL (2002) Trajectories of microwave prominence eruptions. Astron Astrophys 382(2):666-677. https:/doi.org/10.1051/0004-6361:20011658

Hou YJ, Zhang J, Li T, Yang SH, Li XH (2018) Eruption of a Multi-flux-rope system in solar active region 12673 leading to the two largest flares in solar cycle 24. Astron Astrophys 619:A100. https://doi.org/10.1051/0004-6361/201732530

Howard RA, Moses JD, Vourlidas A, Newmark JS, Socker DG, Plunkett SP, Korendyke CM et al (2008) Sun Earth Connection Coronal and Heliospheric Investigation (SECCHI). Space Sci Rev 136:67-115. https://doi.org/10.1007/ s11214-008-9341-4

Howard TA, DeForest CE, Schneck UG, Alden CR (2017) Challenging some contemporary views of coronal mass ejections. II. The case for absent filaments. Astrophys J 834:86. https://doi.org/10.3847/1538-4357/834/1/86

Howard TA, Harrison RA (2013) Stealth coronal mass ejections: a perspective. Sol Phys 285(1):269-280. https://doi.org/10.1007/s11207-012-0217-0

Howard TA, Stovall K, Dowell J, Taylor GB, White SM (2016) Measuring the magnetic field of coronal mass ejections near the sun using pulsarS. Astrophys J 831 (2):208. https://doi.org/10.3847/0004-637X/831/2/208

Howard TA, Tappin SJ (2009) Interplanetary coronal mass ejections observed in the heliosphere: 1. Review of theory. Space Sci Rev 147(1-2):31-54

Hu H, Liu YD, Wang R, Möstl C, Yang Z (2016) Sun-to-Earth characteristics of the 2012 July 12 Coronal mass ejection and associated geo-effectiveness. Astrophys J 829:97. https://doi.org/10.3847/0004-637X/829/2/97
Hu Q (2017) The Grad-Shafranov reconstruction of toroidal magnetic flux ropes: method development and benchmark studies. Sol Phys 292(9):116. https:// doi.org/10.1007/s11207-017-1134-z

Hu Q, Farrugia CJ, Osherovich VA, Möstl C, Szabo A, Ogilvie KW, Lepping RP (2013) Effect of electron pressure on the Grad-Shafranov reconstruction of interplanetary coronal mass ejections. Sol Phys 284(1):275-291. https://doi. org/10.1007/s11207-013-0259-y

Hu Q, Jiong Q, Dasgupta B, Khare A, Webb GM (2014) Structures of interplanetary magnetic flux ropes and comparison with their solar sources. Astrophys J 793:53. https://doi.org/10.1088/0004-637X/793/1/53

Hu Q, Sonnerup BUÖ (2001) Reconstruction of Magnetic flux ropes in the solar wind. Geophys Res Lett 28(3):467-470. https://doi.org/10.1029/2000GL012232

Hu Q, Linton MG, Wood BE, Riley P, Nieves-Chinchilla T (2017) The GradShafranov reconstruction of toroidal magnetic flux ropes: first applications. Sol Phys 292(11):171. https://doi.org/10.1007/s11207-017-1195-z

Hu Q, Sonnerup BUÖ (2002) Reconstruction of magnetic clouds in the solar wind: orientations and configurations. J Geophys Res 107(A7):1142. https:// doi.org/10.1029/2001JA000293

Huang Z, Madjarska MS, Doyle JG, Lamb DA (2012) Coronal hole boundaries at small scales. IV. SOT View. Magnetic field properties of small-scale transient brightenings in coronal holes. Astron Astrophys 548:A62. https://doi.org/10.1 051/0004-6361/201220079

Hudson HS (2011) Global properties of solar flares. Space Sci Rev 158:5-41. https://doi.org/10.1007/s11214-010-9721-4

Hudson HS (2018) The relationship between long-duration gamma-ray flares and solar cosmic rays. 335:49-53. https://doi.org/10.1017/S1743921317009681

Hudson HS, Acton LW, Alexander D, Freeland SL, Lemen JR, Harvey KL (1996) Yohkoh/SXT soft $\mathrm{x}$-ray observations of sudden mass loss from the solar corona. 382:88-91. https://doi.org/10.1063/1.51457

Hudson HS, Cliver EW (2001) Observing coronal mass ejections without coronagraphs. J Geophys Res Space Phys 106(A11):25199-25213. https://doi. org/10.1029/2000JA904026

Huttunen K, Emilia J, Koskinen HEJ, Schwenn R (2002) Variability of magnetospheric storms driven by different solar wind perturbations. J Geophys Res (Space Physics) 107:1121. https://doi.org/10.1029/2001JA900171

Huttunen KEJ, Kilpua SP, Pulkkinen A, Viljanen A, Tanskanen E (2008) Solar wind drivers of large geomagnetically induced currents during the solar cycle 23. Space Weather 6(10):S10002. https://doi.org/10.1029/2007SW000374

Illarionov EA, Tlatov AG (2018) Segmentation of Coronal holes in solar disc images with a convolutional neural network. Mnras 481(4):5014-5021. https://doi.org/10.1093/mnras/sty2628

Illing RME, Hundhausen AJT (1986) Disruption of a Coronal streamer by an eruptive prominence and coronal mass ejection. J Geophys Res Space Phys 91(A10):10951-10960

Inoue S, Hayashi K, Kusano K (2016) Structure and stability of magnetic fields in solar active region 12192 based on the nonlinear force-free field modeling. Astrophys J 818:168. https://doi.org/10.3847/0004-637X/818/2/168

Inoue S, Kusano K, Magara T, Shiota D, Yamamoto TT (2011) Twist and connectivity of magnetic field lines in the solar active region NOAA 10930. Astrophys J 738:161. https://doi.org/10.1088/0004-637X/738/2/161

Inoue S, Shiota D, Bamba Y, Park S-H (2018) Magnetohydrodynamic modeling of a solar eruption associated with an X9.3 flare observed in the active region 12673. Astrophys J 867(1):83. https://doi.org/10.3847/1538-4357/aae079

Isavnin A (2016) FRiED: A novel three-dimensional model of coronal mass ejections. Astrophys J 833(2):267. https://doi.org/10.3847/1538-4357/833/2/267

Isavnin A, Vourlidas A, Kilpua EKJ (2013) Three-dimensional evolution of erupted flux ropes from the Sun $(2-20 \mathrm{R} \odot)$ to 1 AU. Sol Phys 284(1):203-215. https://doi.org/10.1007/s11207-012-0214-3

Isavnin A, Vourlidas A, Kilpua EKJ (2014) Three-dimensional evolution of flux-rope CMEs and its relation to the local orientation of the heliospheric current sheet. Sol Phys 289(6):2141-2156. https://doi.org/10.1007/s11207-013-0468-4

Ishiguro N, Kusano K (2017) Double arc instability in the solar corona. Astrophys J 843:101. https://doi.org/10.3847/1538-4357/aa799b

Iwai K, Shiota D, Tokumaru M, K'i F, Den M, Kubo Y (2021) Validation of coronal mass ejection arrival-time forecasts by magnetohydrodynamic simulations based on interplanetary scintillation observations. Earth Planets Space 73:9. https://doi.org/10.1186/s40623-020-01345-5

Jacobs C, Poedts S (2011) Models for coronal mass ejections. J Atmos Sol Terr Phys 73(10):1 148-1155. https://doi.org/10.1016/j.jastp.2010.12.002 
Jacobs C, Poedts S (2012) A numerical study of the response of the coronal magnetic field to flux emergence. Sol Phys 280(2):389-405. https://doi.org/1 0.1007/s11207-012-9941-8

James AW, Green LM, Palmerio E, Valori G, Reid HAS, Baker D, Brooks DH, van Driel-Gesztelyi L, Kilpua EKJ (2017) On-Disc observations of flux rope formation prior to its eruption. Sol Phys 292:71. https://doi.org/10.1007/s112 07-017-1093-4

Janvier M, Aulanier G, Bommier V, Schmieder B, Démoulin P, Pariat E (2014) Electric currents in flare ribbons: observations and three-dimensional standard model. Astrophys J 788:60. https://doi.org/10.1088/0004-637X/ $788 / 1 / 60$

Janvier M, Aulanier G, Pariat E, Démoulin P (2013a) The standard flare model in three dimensions. III. Slip-running reconnection properties. Astron Astrophys 555:A77. https://doi.org/10.1051/0004-6361/201321164

Janvier M, Démoulin P, Dasso S (2013b) Global axis shape of magnetic clouds deduced from the distribution of their local axis orientation. Astron Astrophys 556:A50. https://doi.org/10.1051/0004-6361/201321442

Janvier M, Winslow RM, Good S, Bonhomme E, Démoulin P, Dasso S, Möstl C et al (2019) Generic magnetic field intensity profiles of interplanetary coronal mass ejections at mercury, venus, and earth from superposed epoch analyses. J Geophys Res (Space Physics) 124(2):812-836. https://doi.org/10.1 029/2018JA025949

Jensen EA, Russell CT (2008) Faraday rotation observations of CMEs. Geophys Res Lett 35(2):L02103. https://doi.org/10.1029/2007GL031038

Ji H, Wang H, Schmahl EJ, Moon Y-J, Jiang Y (2003) Observations of the failed eruption of a filament. Astrophys J Lett 595:L135-L138. https://doi.org/10.1 086/378178

Jian LK, Luhmann JG, Russell CT, Galvin AB (2019) Solar Terrestrial Relations Observatory (STEREO) observations of stream interaction regions in 2007 2016: relationship with heliospheric current sheets, solar cycle variations, and dual observations. Sol Phys 294:31. https://doi.org/10.1007/s11207-019-1416-8

Jian LK, MacNeice P, Taktakishvili A, Odstrcil D, Jackson B, Yu H-S, Riley P, Sokolov I (2015) How reliable is the prediction of solar wind background? 1:200.01 http://adsabs.harvard.edu/abs/2015TESS....120001」

Jian LK, MacNeice PJ, Mays ML, Taktakishvili A, Odstrcil D, Jackson B, Yu H-S, Riley P, Sokolov IV (2016) Validation for global solar wind prediction using Ulysses comparison: multiple coronal and heliospheric models installed at the community coordinated modeling center. Space Weather 14:592-611. https://doi.org/10.1002/2016SW001435

Jian LK, Russell CT, Luhmann JG, Galvin AB, MacNeice PJ (2009) Multi-spacecraft observations: stream interactions and associated structures. Sol Phys 259(12):345-360. https://doi.org/10.1007/s11207-009-9445-3

Jiang C, Feng X (2012) A new implementation of the magnetohydrodynamicsrelaxation method for nonlinear force-free field extrapolation in the solar corona. Astrophys J 749(2):135. https://doi.org/10.1088/0004-637X/749/2/135

Jiang C, Feng X (2014) Preprocessing the photospheric vector magnetograms for an NLFFF extrapolation using a potential-field model and an optimization method. Sol Phys 289(1):63-77. https://doi.org/10.1007/s11207-013-0346-0

Jiang C, Feng X, Fan Y, Xiang C (2011) Reconstruction of the coronal magnetic field using the cese-mhd method. Astrophys J 727(2):101. https://doi.org/10.1 088/0004-637X/727/2/101

Jiang C, Wu ST, Feng X, Hu Q (2013b) Formation and eruption of an active region sigmoid. I. A study by nonlinear force-free field modeling. Astrophys $J$ 780(1):55. https://doi.org/10.1088/0004-637X/780/1/55

Jiang C, Wu ST, Feng X, Qiang H (2014) Nonlinear force-free field extrapolation of a coronal magnetic flux rope supporting a large-scale solar filament from a photospheric vector magnetogram. Astrophys J 786(2):L16. https://doi.org/1 0.1088/2041-8205/786/2/L16

Jiang C, Wu ST, Feng X, Qiang H (2016) Data-Driven Magnetohydrodynamic modelling of a flux-emerging active region leading to solar eruption. Nat Commun 7(1):11522. https://doi.org/10.1038/ncomms11522

Jiang C, Xueshang F, Wu ST, Qiang H (2012) Study of the three-dimensiona coronal magnetic field of active region 11117 around the time of a confined flare using a data-driven CESE-MHD model. Astrophys J 759(2):85. https://doi. org/10.1088/0004-637X/759/2/85

Jiang C, Xueshang F, Wu ST, Qiang H (2013a) Magnetohydrodynamic simulation of a sigmoid eruption of active region 11283. Astrophys J 771(2):L30. https:// doi.org/10.1088/2041-8205/771/2/L30

Jiang C, Yan X, Feng X, Duan A, Hu Q, Zuo P, Wang Y (2017) Reconstruction of a large-scale pre-flare coronal current sheet associated with a homologous $\mathrm{X}$ shaped flare. Astrophys J 850(1):8. https://doi.org/10.3847/1538-4357/aa917a
Jiang C, Zou P, Feng X, Hu Q, Rui L, Vemareddy P, Duan A, Zuo P, Wang Y, Wei F (2018) Magnetohydrodynamic Simulation of the X9.3 Flare on 2017 September 6: Evolving magnetic topology. Astrophys J 869(1):13. https://doi. org/10.3847/1538-4357/aaeacc

Jiggens P, Chavy-Macdonald M-A, Santin G, Menicucci A, Evans H, Hilgers A (2014) The magnitude and effects of extreme solar particle events. J Space Weather Space Climate 4:A20. https://doi.org/10.1051/swsc/2014017

Jin M, Manchester WB, van der Holst B, Oran R, Sokolov I, Toth G, Liu Y, Sun XD, Gombosi TI (2013) Numerical simulations of coronal mass ejection on 2011 MARCH 7: one-temperature and two-temperature model comparison. Astrophys J 773(1):50. https://doi.org/10.1088/0004-637X/773/1/50

Jin M, Manchester WB, van der Holst B, Sokolov I, Tóth G, Mullinix RE, Taktakishvili A, Chulaki A, Gombosi TI (2017a) Data-constrained coronal mass ejections in a global magnetohydrodynamics modeL. Astrophys J 834(2):173. https://doi. org/10.3847/1538-4357/834/2/173

Jin M, Manchester WB, van der Holst B, Sokolov I, Tóth G, Vourlidas A, de Koning CA, Gombosi TI (2017b) Chromosphere to 1 Au simulation of the 2011 March 7th event: a comprehensive study of coronal mass ejection propagation. Astrophys J 834(2):172. https://doi.org/10.3847/1538-4357/ 834/2/172

Jin M, Schrijver CJ, Cheung MCM, DeRosa ML, Nitta NV, Title AM (2016) A numerical study of long-range magnetic impacts during coronal mass ejections. Astrophys J 820(1):16. https://doi.org/10.3847/0004-637X/820/1/16

Jing J, Liu C, Lee J, Ji H, Liu N, Xu Y, Wang H (2018) Statistical analysis of torus and kink instabilities in solar eruptions. Astrophys J 864(2):138. https://doi. org/10.3847/1538-4357/aad6e4

Kahler S (1977) The morphological and statistical properties of solar x-ray events with long decay times. Astrophys J 214:891-897. https://doi.org/10.1086/1 55319

Kahler SW (2001) The correlation between solar energetic particle peak intensities and speeds of coronal mass ejections: effects of ambient particle intensities and energy spectra. J Geophys Res Space Phys 106(A10):20947-20955

Kahler SW, Haggerty DK, Richardson IG (2011) Magnetic field-line lengths in interplanetary coronal mass ejections inferred from energetic electron events. Astrophys J 736(2):106

Kahler SW, Tylka AJ, Reames DV (2009) A comparison of elemental abundance ratios in SEP events in fast and slow solar wind regions. Astrophys J 701(1):561

Kahler SW, Vourlidas A (2013) A comparison of the intensities and energies of gradual solar energetic particle events with the dynamical properties of associated coronal mass ejections. Astrophys J 769(2):143

Kaiser ML, Kucera TA, Davila JM, Cyr OCS, Guhathakurta M, Christian E (2008) The STEREO mission: an introduction. Space Sci Rev 136:5-16. https://doi.org/10.1 007/s11214-007-9277-0

Karachik NV, Pevtsov AA (2011) Solar wind and coronal bright points inside coronal holes. Apj 735(1):47. https://doi.org/10.1088/0004-637X/735/1/47

Kataoka R, Ebisuzaki T, Kusano K, Shiota D, Inoue S, Yamamoto TT, Tokumaru M (2009) Three-dimensional MHD modeling of the solar wind structures associated with 13 December 2006 coronal mass ejection: MHD modeling of an interplanetary CME. J Geophys Res Space Phys 114(A10):-n/a. https://doi. org/10.1029/2009JA014167

Kay C, Gopalswamy N (2018) The effects of uncertainty in initial CME input parameters on deflection, rotation, $\mathrm{B}_{\mathrm{z}}$ and arrival time predictions. J Geophys Res 123(9):7220-7240. https://doi.org/10.1029/2018JA025780

Kay C, Gopalswamy N, Reinard A, Opher M (2017) Predicting the magnetic field of Earth-impacting CMEs. Astrophys J 835(2):117. https://doi.org/10.3847/153 8-4357/835/2/117

Kay C, Mays ML, Verbeke C (2020a) Identifying critical input parameters for improving drag-based CME arrival time predictions. Space Weather 18: e02382. https://doi.org/10.1029/2019SW002382

Kay C, Nieves-Chinchilla T, Jian LK (2020b) FIDO-SIT: the first forward model for the in situ magnetic field of CME-driven sheaths. J Geophys Res (Space Physics) 125:e27423. https://doi.org/10.1029/2019JA027423

Kay C, Opher M (2015) The heliocentric distance where the deflections and rotations of solar coronal mass ejections occur. Astrophys J 811(2):L36. https://doi.org/10.1088/2041-8205/811/2/L36

Kay C, Opher M, Evans RM (2013) Forecasting a coronal mass ejection's altered trajectory: ForeCAT. Astrophys J 775:5. https://doi.org/10.1088/0004-637X/ $775 / 1 / 5$

Kazachenko MD, Lynch BJ, Welsch BT, Sun X (2017) A database of flare ribbon properties from the solar dynamics observatory. I. Reconnection flux. Astrophys J 845:49. https://doi.org/10.3847/1538-4357/aa7ed6 
Keppens R, Meliani Z, van Marle AJ, Delmont P, Vlasis A, van der Holst B (2012) Parallel, grid-adaptive approaches for relativistic hydro and magnetohydrodynamics. J Comput Phys 231(3):718-744. https://doi.org/10.1 016/j.jcp.2011.01.020

Kilpua E, Koskinen HEJ, Pulkkinen TI (2017a) Coronal Mass ejections and their sheath regions in interplanetary space. Living Rev Sol Phys 14:5. https://doi. org/10.1007/s41116-017-0009-6

Kilpua EKJ, Balogh A, von Steiger R, Liu YD (2017) Geoeffective properties of solar transients and stream interaction regions. Space Sci Rev 212:1271-1314. https://doi.org/10.1007/s11214-017-0411-3

Kilpua EKJ, Lugaz N, Mays ML, Temmer M (2019) Forecasting the structure and orientation of Earthbound coronal mass ejections. Space Weather 17:498526. https://doi.org/10.1029/2018SW001944

Kilpua EKJ, Lumme E, Andreeova K, Isavnin A, Koskinen HEJ (2015) Properties and drivers of fast interplanetary shocks near the orbit of the Earth (1995-2013). J Geophys Res (Space Physics) 120(6):4112-4125. https://doi.org/10.1002/201 5JA021138

Kilpua EK, Mierla M, Rodriguez L, Zhukov AN, Srivastava N, West MJ (2012) Estimating travel times of coronal mass ejections to $1 \mathrm{AU}$ using multispacecraft coronagraph data. Sol Phys 279:477-496. https://doi.org/10.1007/ s11207-012-0005-x

Kilpua EKJ, Mierla M, Zhukov AN, Rodriguez L, Vourlidas A, Wood B (2014) Solar sources of interplanetary coronal mass ejections during the solar cycle 23/24 minimum. Sol Phys 289:3773-3797. https://doi.org/10.1007/s11207-0140552-4

Kilpua EKJ, Pomoell J, Vourlidas A, Vainio R, Luhmann J, Li Y, Schroeder P, Galvin $A B$, Simunac K (2009) STEREO observations of interplanetary coronal mass ejections and prominence deflection during solar minimum period. Ann Geophys 27:4491-4503. https://doi.org/10.5194/angeo-27-4491-2009

Klassen A, Aurass H, Mann G, Thompson BJ (2000) Catalogue of the 1997 SOHOEIT coronal transient waves and associated type II radio burst spectra. Astron Astrophys Suppl Ser 141(3):357-369. https://doi.org/10.1051/aas:2000125

Klassen A, Dresing N, Gómez-Herrero R, Heber B, Müller-Mellin R (2016) Unexpected spatial intensity distributions and onset timing of solar electron events observed by closely spaced STEREO spacecraft. Astron Astrophys 593:A31

Klassen A, Dresing N, Gómez-Herrero R, Heber B, Veronig A (2018) Strong nonradial propagation of energetic electrons in solar corona. Astron Astrophys 614:A61

Klecker B, Möbius E, Popecki MA (2007) lonic charge states of solar energetic particles: a clue to the source. Space Sci Rev 130(1-4):273-282

Klein K-L, Dalla S (2017) Acceleration and propagation of solar energetic particles. Space Sci Rev 212(3):1107-1136. https://doi.org/10.1007/s11214-017-0382-4

Klein K-L, Krucker S, Lointier G, Kerdraon A (2008) Open magnetic flux tubes in the corona and the transport of solar energetic particles. Astron Astrophys 486(2):589-596

Klein K-L, Posner A (2005) The onset of solar energetic particle events: prompt release of Deka-MeV protons and associated coronal activity. Astron Astrophys 438(3):1029-1042

Klein K-L, Tziotziou K, Zucca P, Valtonen E, Vilmer N, Malandraki OE, Hamadache C, Heber B, Kiener J (2018) X-Ray, radio and SEP observations of relativistic gamma-ray events. In: Solar Particle Radiation Storms Forecasting and Analysis. Springer, Cham, pp 133-155

Kliem B, Lin J, Forbes TG, Priest ER, Török T (2014) Catastrophe versus instability for the eruption of a toroidal solar magnetic flux rope. Astrophys J 789(1):46. https://doi.org/10.1088/0004-637X/789/1/46

Kliem B, Török T (2006) Torus instability. Phys Rev Lett 96(25):255002. https://doi org/10.1103/PhysRevLett.96.255002

Klimchuk JA (2001) Theory of coronal mass ejections. Washington DC Am Geophys Union GeophysMonogr Ser 125. https://doi.org/10.1029/GM12 5 p0143

Knipp DJ, Ramsay AC, Beard ED, Boright AL, Cade WB, Hewins IM, McFadden RH et al (2016) The May 1967 great storm and radio disruption event: extreme space weather and extraordinary responses. Space Weather 14(9):614-633. https://doi.org/10.1002/2016SW001423

Kocharov L, Laitinen T, Vainio R, Afanasiev A, Mursula K, Ryan JM (2015) Solar interacting protons versus interplanetary protons in the core plus halo model of diffusive shock acceleration and stochastic re-acceleration. Astrophys J 806(1):80

Kong X, Guo F, Chen Y, Giacalone J (2019) The Acceleration of energetic particles at coronal shocks and emergence of a double power-law feature in particle energy spectra. Astrophys J 883(1):49
Kong X, Guo F, Giacalone J, Li H, Chen Y (2017) The acceleration of high-energy protons at coronal shocks: the effect of large-scale streamer-like magnetic field structures. Astrophys J 851(1):38

Kontogiannis I, Georgoulis MK, Guerra JA, Park S-H, Shaun Bloomfield D (2019) Which photospheric characteristics are most relevant to active-region coronal mass ejections? Sol Phys 294:130. https://doi.org/10.1007/s11207-019-1523-6

Kooi JE, Ascione ML, Reyes-Rosa LV, Rier SK, Ashas M (2021) VLA measurements of faraday rotation through a coronal mass ejection using multiple lines of sight. Sol Phys 296:11. https://doi.org/10.1007/s11207-020-01755-4

Kooi JE, Fischer PD, Buffo JJ, Spangler SR (2017) VLA Measurements of Faraday rotation through coronal mass ejections. Sol Phys 292(4):56. https://doi.org/1 0.1007/s11207-017-1074-7

Kopp RA, Pneuman GW (1976) Magnetic reconnection in the corona and the loop prominence phenomenon. Sol Phys 50:85-98. https://doi.org/10.1007/ BF00206193

Kosugi T, Matsuzaki K, Sakao T, Shimizu T, Sone Y, Tachikawa S, Hashimoto T et al (2007) The Hinode (Solar-B) mission: an overview. Sol Phys 243:3-17. https:// doi.org/10.1007/s11207-007-9014-6

Kota J (1979) Some implications of the higher harmonics of galactic anisotropy. Kozponti Fizikai Kutato Intezet

Kouloumvakos A, Patsourakos S, Nindos A, Vourlidas A, Anastasiadis A, Hillaris A, Sandberg I (2016) Multi-viewpoint observations of a widely distributed solar energetic particle event: the role of Euv waves and white-light shock signatures. Astrophys J 821(1):31

Kouloumvakos A, Rouillard AP, Wu Y, Vainio R, Vourlidas A, Plotnikov I, Afanasiev A, Önel H (2019) Connecting the properties of coronal shock waves with those of solar energetic particles. Astrophys J 876(1):80

Kozarev KA, Raymond JC, Lobzin W, Hammer M (2015) Properties of a coronal shock wave as a driver of early SEP acceleration. Astrophys J 799(2):167

Krauss S, Temmer M, Vennerstrom S (2018) Multiple satellite analysis of the Earth's thermosphere and interplanetary magnetic field variations due to ICME/CIR events during 2003-2015. J Geophys Res (Space Physics) 123(10): 8884-8894. https://doi.org/10.1029/2018JA025778

Krauss S, Temmer M, Veronig A, Baur O, Lammer H (2015) Thermospheric and geomagnetic responses to interplanetary coronal mass ejections observed by ACE and GRACE: statistical results. J Geophys Res (Space Physics) 120(10): 8848-8860. https://doi.org/10.1002/2015JA021702

Krista LD (2012) The evolution and space weather effects of solar coronal holes. PhD Thesis

Krivova NA, Solanki SK (2008) Models of solar irradiance variations: current status. J Astrophys Astron 29:151-158. https://doi.org/10.1007/s12036-008-0018-x

Kubicka M, Möstl C, Amerstorfer T, Boakes PD, Feng L, Eastwood JP, Törmänen O (2016) Prediction of geomagnetic storm strength from inner heliospheric in situ observations. Astrophys J 833(2):255. https://doi.org/10.3847/1538-4357/ $833 / 2 / 255$

Kumar A, Rust DM (1996) Interplanetary magnetic clouds, helicity conservation, and current-core flux-ropes. J Geophys Res 101:15667-15684. https://doi. org/10.1029/96JA00544

Kunkel V, Chen J (2010) Evolution of a coronal mass ejection and its magnetic field in interplanetary space. Astrophys J 715(2):L80-L83. https://doi.org/10.1 088/2041-8205/715/2/L80

Kusano K, lju T, Bamba Y, Inoue S (2020) A physics-based method that can predict imminent large solar flares. Science 369:587-591. https://doi.org/1 $0.1126 /$ science.aaz2511

Kwon R-Y, Zhang J, Olmedo O (2014) New insights into the physical nature of coronal mass ejections and associated shock waves within the framework of the three-dimensional structure. Astrophys J 794(2):148

Kwon R-Y, Zhang J, Vourlidas A (2015) Are halo-like solar coronal mass ejections merely a matter of geometric projection effects? Astrophys J Lett 799:L29. https://doi.org/10.1088/2041-8205/799/2/L29

LaBonte BJ, Georgoulis MK, Rust DM (2007) Survey of magnetic helicity injection in regions producing X-class flares. Astrophys J 671:955-963. https://doi.org/1 $0.1086 / 522682$

Laitinen T, Dalla S (2019) From Sun to interplanetary space: what is the pathlength of solar energetic particles? Astrophys J 887(2):222. https://doi. org/10.3847/1538-4357/ab54c7

Laitinen T, Kopp A, Effenberger F, Dalla S, Marsh MS (2016) Solar energetic particle access to distant longitudes through turbulent field-line meandering. Astron Astrophys 591:A18. https://doi.org/10.1051/0004-6361/201527801

Laming JM (2015) The FIP and inverse FIP effects in solar and stellar coronae. Living Rev Sol Phys 12(1):2 
Lamy PL, Floyd O, Boclet B, Wojak J, Gilardy H, Barlyaeva T (2019) Coronal mass ejections over solar cycles 23 and 24. Space Sci Rev 215:39. https://doi.org/1 0.1007/s11214-019-0605-y

Landi E, Gruesbeck JR, Lepri ST, Zurbuchen TH, Fisk LA (2012) Charge state evolution in the solar wind. II. Plasma charge state composition in the inner corona and accelerating fast solar wind. Astrophys J 761(1):48. https://doi. org/10.1088/0004-637X/761/1/48

Lanzerotti LJ (2017) Space weather: historical and contemporary perspectives. Space Sci Rev 212:1253-1270. https://doi.org/10.1007/s11214-017-0408-y

Lario D, Aran A, Gómez-Herrero R, Dresing N, Heber B, Ho GC, Decker RB, Roelof EC (2013) Longitudinal and radial dependence of solar energetic particle peak intensities: STEREO, ACE, SOHO, GOES, and MESSENGER observations. Astrophys J 767(1):41

Lario D, Hu Q, Ho GC, Decker RB, Roelof EC, Smith CW (2005) Statistical properties of fast forward transient interplanetary shocks and associated energetic particle events: ACE observations. In: Solar Wind 11/SOHO 16, Connecting Sun and Heliosphere, vol 592, p 81

Lario D, Kwon R-Y, Vourlidas A, Raouafi NE, Haggerty DK, Ho GC, Anderson BJ et al (2016) Longitudinal properties of a widespread solar energetic particle event on 2014 February 25: evolution of the associated CME shock. Astrophys J 819(1):72

Lario D, Raouafi NE, Kwon R-Y, Zhang J, Gómez-Herrero R, Dresing N, Riley P (2014) The solar energetic particle event on 2013 April 11: an investigation of its solar origin and longitudinal spread. Astrophys J 797:8. https://doi.org/10.1 088/0004-637X/797/1/8

Larson DE, Lin RP, McTiernan JM, McFadden JP, Ergun RE, McCarthy M, Rème H et al (1997) Tracing the topology of the October 18-20, 1995, magnetic cloud with $\sim 0.1-10^{2} \mathrm{KeV}$ electrons. Geophys Res Lett 24(15):1911-1914. https://doi. org/10.1029/97GL0e1878

Laurenza M, Consolini G, Storini M, Damiani A (2013) On the spectral shape of SEP events: an extreme value statistics approach. In: AIP Conference Proceedings, vol 1539. American Institute of Physics, pp 219-222

Laurenza M, Consolini G, Storini M, Damiani A (2015) The Weibull functional form for SEP event spectra. In: Journal of Physics: Conference Series, vol 632. IOP Publishing, p 012066

Laurenza M, Consolini G, Storini M, Pallocchia G, Damiani A (2016) The Weibull functional form for the energetic particle spectrum at interplanetary shock waves. In: Journal Of Physics: Conference Series (СМ. В КНИГАХ) vol 767. Institute of Physics and IOP Publishing Limited, p 012015

Lavraud B, Ruffenach A, Rouillard AP, Kajdic P, Manchester WB, Lugaz N (2014) Geo-effectiveness and radial dependence of magnetic cloud erosion by magnetic reconnection: magnetic cloud erosion geo-effectiveness. J Geophys Res Space Phys 119(1):26-35. https:/doi.org/10.1002/2013JA019154

Le G-M, Li C, Zhang X-F (2017) Dependence of E $\geq 100 \mathrm{MeV}$ protons on the associated flares and CMEs. Res Astron Astrophys 17(7):073

Le G-M, Zhang X-F (2017) Dependence of large SEP Events with different energies on the associated flares and CMEs. Res Astron Astrophys 17(12):123

Lean J (1991) Variations in the Sun's radiative output. Rev Geophys 29(4):505-535. https://doi.org/10.1029/91RG01895

Lee CO, Luhmann JG, Odstrcil D, MacNeice PJ, de Pater I, Riley P, Arge CN (2009) The solar wind at $1 \mathrm{AU}$ during the declining phase of solar cycle 23: comparison of 3D numerical model results with observations. Sol Phys 254: 155-183. https://doi.org/10.1007/s11207-008-9280-y

Lee MA (1983) Coupled hydromagnetic wave excitation and ion acceleration at interplanetary traveling shocks. J Geophys Res Space Phys 88(A8):6109-6119

Lee MA (2005) Coupled hydromagnetic wave excitation and ion acceleration at an evolving coronal/interplanetary shock. Astrophys J Suppl Ser 158(1):38

Leka KD, Park S-H, Kusano K, Andries J, Barnes G, Bingham S, Bloomfield DS et al (2019a) A comparison of flare forecasting methods. II. Benchmarks, metrics, and performance results for operational solar flare forecasting systems. Astrophys J Suppl Ser 243(2):36. https://doi.org/10.3847/1538-4365/ab2e12

Leka KD, Park S-H, Kusano K, Andries J, Barnes G, Bingham S, Bloomfield DS et al (2019b) A comparison of flare forecasting methods. III. Systematic behaviors of operational solar flare forecasting systems. Astrophys J 881:101. https://doi. org/10.3847/1538-4357/ab2e11

Lepping RP (2003) Estimated errors in magnetic cloud model fit parameters with force-free cylindrically symmetric assumptions. J Geophys Res 108(A10):1356. https://doi.org/10.1029/2002JA009657

Lepping RP, Berdichevsky DB, Wu C-C (2017) Average magnetic field magnitude profiles of wind magnetic clouds as a function of closest approach to the clouds' axes and comparison to model. Sol Phys 292(2):27. https://doi.org/1 0.1007/s11207-016-1040-9

Lepping RP, Burlaga LF, Jones JA (1990) Magnetic field structure of interplanetary magnetic clouds at 1 AU. J Geophys Res 95:11957-11965. https://doi.org/10.1 029/JA095iA08p11957

Lepping RP, Wu C-C (2007) On the variation of interplanetary magnetic cloud type through solar cycle 23: wind events. J Geophys Res (Space Physics) 112: A10103. https://doi.org/10.1029/2006JA012140

Lepping RP, Wu C-C, Berdichevsky DB, Kay C (2018) Magnetic field magnitude modification for a force-free magnetic cloud model. Sol Phys 293(12):162. https://doi.org/10.1007/s11207-018-1383-5

Leske RA, Cohen CMS, Mewaldt RA, Christian ER, Cummings AC, Labrador AW, Stone EC, Wiedenbeck ME, von Rosenvinge TT (2012) Large proton anisotropies in the 18 August 2010 solar particle event. Sol Phys 281(1):301-318

Li G, Lee MA (2015) Scatter-dominated interplanetary transport of solar energetic particles in large gradual events and the formation of double power-law differential fluence spectra of ground-level events during solar cycle 23. Astrophys J 810(1):82

Li G, Moore R, Mewaldt RA, Zhao L, Labrador AW (2012) A twin-CME scenario for ground level enhancement events. Space Sci Rev 171(1-4):141-160

Li G, Zank GP, Olga V, Mewaldt RA, Cohen CMS, Mason GM, Desai MI (2009a) Shock geometry and spectral breaks in large SEP events. Astrophys J 702(2): 998

Li H, Feng X (2018) CESE-HLL magnetic field-driven modeling of the background solar wind during year 2008. J Geophys Res Space Phys 123(6):4488-4509. https://doi.org/10.1029/2017JA025125

Li HJ, Feng XS, Zuo PB, Xie YQ (2009b) Inferring Interplanetary flux rope orientation with the minimum residue method. J Geophys Res (Space Physics) 114(A3):A03102. https://doi.org/10.1029/2008JA013331

Li Y, Luhmann JG, Lynch BJ (2018) Magnetic clouds: solar cycle dependence, sources, and geomagnetic impacts. Textbackslashsolphys 293(10):135

Liewer P, Panasenco O, Vourlidas A, Colaninno R (2015) Observations and analysis of the non-radial propagation of coronal mass ejections near the Sun. Sol Phys 290:3343-3364. https://doi.org/10.1007/s11207-015-0794-9

Lin J, Soon W, Baliunas SL (2003) Theories of solar eruptions: a review. New Astron Rev 47:53-84. https://doi.org/10.1016/S1387-6473(02)00271-3

Lin PH, Kusano K, Shiota D, Satoshi I, Leka KD, Mizuno Y (2020) A new parameter of the photospheric magnetic field to distinguish eruptive-flare producing solar active regions. Astrophys J 894:20. https://doi.org/10.3847/1538-4357/a b822c

Linker JA, Caplan RM, Downs C, Lionello R, Riley P, Mikic Z, Henney CJ, Arge CN, Kim T, Pogorelov N (2016) An empirically driven time-dependent model of the solar wind. J Phys Conf Ser 719:012012. https://doi.org/10.1088/17426596/719/1/012012

Lionello R, Downs C, Linker JA, Török T, Riley P, Mikić Z (2013) Magnetohydrodynamic simulations of interplanetary coronal mass ejections. Astrophys J 777(1):76. https://doi.org/10.1088/0004-637X/777/1/76

Lionello R, Linker JA, Mikić Z (2008) Multispectral emission of the Sun during the first whole Sun month: magnetohydrodynamic simulations. Astrophys J 690(1):902

Liou K, Wu C-C, Dryer M, Wu S-T, Rich N, Plunkett S, Simpson L, Fry CD, Schenk K (2014) Global simulation of extremely fast coronal mass ejection on 23 July 2012. J Atmos Sol Terr Phys 121:32-41. https://doi.org/10.1016/j.jastp.2014.09.013

Liu J, Ye Y, Shen C, Wang Y, Erdélyi R (2018a) A new tool for CME arrival time prediction using machine learning algorithms: CAT-PUMA. Astrophys J 855(2):109. https://doi.org/10.3847/1538-4357/aaae69

Liu L, Cheng X, Wang Y, Zhou Z, Yang G, Cui J (2018b) Rapid buildup of a magnetic flux rope during a confined X2.2 class flare in NOAA AR 12673 Astrophys J 867(1):L5. https://doi.org/10.3847/2041-8213/aae826

Liu M, Liu YD, Yang Z, Wilson LB, Huidong H (2018c) Kinetic properties of an interplanetary shock propagating inside a coronal mass ejection. Astrophys J 859(1):L4. https://doi.org/10.3847/2041-8213/aac269

Liu Y, Richardson JD, Belcher JW, Wang C, Hu Q, Kasper JC (2006) Constraints on the global structure of magnetic clouds: transverse size and curvature. J Geophys Res 111(A10):A12S03. https://doi.org/10.1029/2006JA011890

Liu Y (2008) Magnetic field overlying solar eruption regions and kink and torus instabilities. Astrophys J Lett 679:L151. https://doi.org/10.1086/589282

Liu Y, Davies JA, Luhmann JG, Vourlidas A, Bale SD, Lin RP (2010) Geometric triangulation of imaging observations to track coronal mass ejections continuously out to $1 \mathrm{AU}$. Astrophys J Lett 710:L82-L87. https://doi.org/10.1 088/2041-8205/710/1/L82 
Liu Y, Shen F, Yang Y (2019b) Numerical Simulation on the propagation and deflection of fast coronal mass ejections (CMEs) Interacting with a corotating interaction region in interplanetary space. Astrophys J 887(2):150. https://doi. org/10.3847/1538-4357/ab543e

Liu YA, Liu YD, Hu H, Wang R, Zhao X (2018d) Multi-spacecraft observations of the rotation and nonradial motion of a CME flux rope causing an intense geomagnetic storm. Astrophys J 854(2):126. https://doi.org/10.3847/1538-43 57/aaa959

Liu YD, Hu H, Wang C, Luhmann JG, Richardson JD, Yang Z, Wang R (2016) On Sun-to-Earth propagation of coronal mass ejections: II. Slow events and comparison with others. Astrophys J Suppl Ser 222:23. https://doi.org/10.384 7/0067-0049/222/2/23

Liu YD, Hu H, Wang R, Yang Z, Zhu B, Liu YA, Luhmann JG, Richardson JD (2015) Plasma and magnetic field characteristics of solar coronal mass ejections in relation to geomagnetic storm intensity and variability. Astrophys J Lett 809: L34. https://doi.org/10.1088/2041-8205/809/2/L34

Liu YD, Hu H, Zhu B, Luhmann JG, Vourlidas A (2017a) Structure, propagation, and expansion of a CME-driven shock in the heliosphere: a revisit of the 2012 July 23 extreme storm. Astrophys J 834(2):158. https://doi.org/10.3847/1 538-4357/834/2/158

Liu YD, Luhmann JG, Kajdič P, Kilpua EKJ, Lugaz N, Nitta NV, Möstl C et al (2014) Observations of an extreme storm in interplanetary space caused by successive coronal mass ejections. Nat Commun 5:3481. https://doi.org/10.1 038/ncomms4481

Liu YD, Luhmann JG, Lugaz N, Möstl C, Davies JA, Bale SD, Lin RP (2013) On Sunto-Earth propagation of coronal mass ejections. Astrophys J 769:45. https:// doi.org/10.1088/0004-637X/769/1/45

Liu YD, Luhmann JG, Möstl C, Martinez-Oliveros JC, Bale SD, Lin RP, Harrison RA, Temmer M, Webb DF, Odstrcil D (2012) Interactions between coronal mass ejections viewed in coordinated imaging and in situ observations. Astrophys J 746(2):L15. https://doi.org/10.1088/2041-8205/746/2/L15

Liu YD, Zhao X, Zhu B (2017b) Propagation and interaction properties of successive coronal mass ejections in relation to a complex type II radio burst. Astrophys J 849(2):112. https://doi.org/10.3847/1538-4357/aa9075

Liu YD, Zhu B, Zhao X (2019a) Geometry, kinematics, and heliospheric impact of a large CME-driven shock in 2017 September. Astrophys J 871(1):8. https:// doi.org/10.3847/1538-4357/aaf425

Lodders K, Palme H, Gail HP (2009) Abundances of the Elements in the Solar System. Landolt Blörnstein 4B:712. https://doi.org/10.1007/978-3-540-88055-4\_34

Long DM, Bloomfield DS, Chen PF, Downs C, Gallagher PT, Kwon R-Y, Vanninathan K et al (2016) Understanding the physical nature of coronal 'EIT Waves. Sol Phys 292(1):7. https://doi.org/10.1007/s11207-016-1030-y

López FM, Cremades H, Balmaceda LA, Nuevo FA, Vásquez AM (2019) Estimating the mass of CMEs from the analysis of EUV dimmings. Astron Astrophys 627: A8. https://doi.org/10.1051/0004-6361/201834163

Lugaz N (2010) Accuracy and limitations of fitting and stereoscopic methods to determine the direction of coronal mass ejections from heliospheric imagers observations. Sol Phys 267:411-429. https://doi.org/10.1007/s11207-010-9654-9

Lugaz N, Farrugia CJ (2014) A new class of complex ejecta resulting from the interaction of two CMEs and its expected geoeffectiveness. Geophys Res Lett 41(3):769-776. https://doi.org/10.1002/2013GL058789

Lugaz N, Farrugia CJ, Davies JA, Möstl C, Davis CJ, Roussev II, Temmer M (2012) The deflection of the two interacting coronal mass ejections of 2010 May 2324 as revealed by combined in situ measurements and heliospheric imaging. Astrophys J 759(1):68. https://doi.org/10.1088/0004-637X/759/1/68

Lugaz N, Farrugia CJ, Huang C-L, Spence HE (2015a) Extreme geomagnetic disturbances due to shocks within CMEs: geomagnetic effects of shocks inside CME. Geophys Res Lett 42(12):4694-4701. https://doi.org/10.1002/201 $5 G L 064530$

Lugaz N, Farrugia CJ, Manchester WB IV, Schwadron N (2013) The interaction of two coronal mass ejections: influence of relative orientation. Astrophys J 778(1):20. https://doi.org/10.1088/0004-637X/778/1/20

Lugaz N, Farrugia CJ, Smith CW, Paulson K (2015b) Shocks inside CMEs: a survey of properties from 1997 to 2006. J Geophys Res Space Phys 120(4):2409_ 2427. https://doi.org/10.1002/2014JA020848

Lugaz N, Farrugia CJ, Winslow RM, Al-Haddad N, Kilpua EKJ, Riley P (2016) Factors affecting the geoeffectiveness of shocks and sheaths at $1 \mathrm{AU}$. J Geophys Res (Space Physics) 121:10. https://doi.org/10.1002/2016JA023100

Lugaz N, Hernandez-Charpak JN, Roussev II, Davis CJ, Vourlidas A, Davies JA (2010a) Determining the azimuthal properties of coronal mass ejections from multi-spacecraft remote-sensing observations with STEREO SECCHI. Astrophys J 715:493-499. https://doi.org/10.1088/0004-637X/715/1/493

Lugaz N, Manchester WB IV, Gombosi TI (2005) Numerical simulation of the interaction of two coronal mass ejections from Sun to Earth. Astrophys J 634(1):651-662. https://doi.org/10.1086/491782

Lugaz N, Roussev II (2011) Numerical Modeling of interplanetary coronal mass ejections and comparison with heliospheric images. J Atmos Sol Terr Phys 73(10):1187-1200. https://doi.org/10.1016/j.jastp.2010.08.016

Lugaz N, Roussev II, Sokolov IV, Jacobs C, Maksimovic M, Issautier K, Meyer-Vernet N, Moncuquet M, Pantellini F (2010b) Solar-Terrestrial Simulations of CMEs with a realistic initiation mechanism: case study for active region. 10069:440441. Saint-Malo, (France). https://doi.org/10.1063/1.3395898

Lugaz N, Temmer M, Wang Y, Farrugia CJ (2017) The interaction of successive coronal mass ejections: a review. Sol Phys 292(4):64. https://doi.org/10.1007/ s11207-017-1091-6

Lugaz N, Vourlidas A, Roussev II (2009) Deriving the radial distances of wide coronal mass ejections from elongation measurements in the heliosphere application to CME-CME interaction. Ann Geophys 27(9):3479-3488. https:// doi.org/10.5194/angeo-27-3479-2009

Luhmann JG, Gopalswamy N, Jian LK, Lugaz N (2020) ICME evolution in the inner heliosphere. Sol Phys 295:61. https://doi.org/10.1007/s11207-020-01624-0

Lundquist S (1951) On the Stability of Magneto-Hydrostatic Fields. Phys Rev 83: 307-311. https://doi.org/10.1103/PhysRev.83.307

Lynch BJ, Antiochos SK, Li Y, Luhmann JG, DeVore CR (2009) Rotation of coronal mass ejections during eruption. Astrophys J 697(2):1918-1927. https://doi. org/10.1088/0004-637X/697/2/1918

Lynch BJ, Masson S, Li Y, DeVore CR, Luhmann JG, Antiochos SK, Fisher GH (2016) A model for stealth coronal mass ejections. J Geophys Res (Space Physics) 121:10. https://doi.org/10.1002/2016JA023432

Ma S, Attrill GDR, Golub L, Lin J (2010) Statistical study of coronal mass ejections with and without distinct low coronal signatures. Astrophys J 722(1):289-301. https://doi.org/10.1088/0004-637X/722/1/289

MacQueen RM, Hundhausen AJ, Conover CW (1986) The propagation of coronal mass ejection transients. J Geophys Res 91:31-38. https://doi.org/10.1029/JA $091 \mathrm{iA01p00031}$

Madjarska MS, Huang Z, Doyle JG, Subramanian S (2012) Coronal hole boundaries evolution at small scales. III. EIS and SUMER views. Astron Astrophys 545:A67. https://doi.org/10.1051/0004-6361/201219516

Madjarska MS, Wiegelmann T (2009) Coronal hole boundaries evolution at small scales. I. EIT $195 \AA$ and TRACE $171 \AA$ view. Astron Astrophys 503:991-997. https://doi.org/10.1051/0004-6361/200912066

Malandraki OE, Crosby NB (2018b) The HESPERIA HORIZON 2020 project and book on solar particle radiation storms forecasting and analysis. Space Weather 16:591-592. https://doi.org/10.1029/2018SW001950

Malandraki OE, Lario D, Lanzerotti LJ, Sarris ET, Geranios A, Tsiropoula G (2005) Violent Sun-Earth connection events of October-November 2003-A09S06October/November 2003 interplanetary coronal mass ejections: ACE/EPAM solar energetic particle observations. J Geophys Res Part A Space Phys 110(9)

Malandraki OE, Sarris ET, Lanzerotti LJ, Trochoutsos P, Tsiropoula G, Pick M (2002) Solar energetic particles inside a coronal mass ejection event observed with the ACE spacecraft. J Atmos Sol Terr Phys 64(5-6):517-525

Malandraki OE, Crosby NB (2018a) Solar energetic particles and space weather: science and applications. In: Malandraki OE, Crosby NB (eds) Solar particle radiation storms forecasting and analysis: the HESPERIA HORIZON 2020 project and beyond. Springer International Publishing, Cham, pp 1-26. Astrophysics and Space Science Library. https://doi.org/10.1007/978-3-319$60051-2 \_1$

Manchester W, Kilpua EKJ, Liu YD, Lugaz N, Riley P, Török T, Vršnak B (2017) The physical processes of CME/ICME evolution. Space Sci Rev 212(3-4):11591219. https://doi.org/10.1007/s11214-017-0394-0

Manchester WB, Gombosi TI, Roussev I, Ridley A, de Zeeuw DL, Sokolov IV, Powell KG, Tóth GB (2004) Modeling a space weather event from the Sun to the Earth: CME generation and interplanetary propagation. J Geophys Res(Space Physics) 109:A02107. https://doi.org/10.1029/2003JA010150

Manchester WB, Kozyra JU, Lepri ST, Lavraud B (2014) Simulation of magnetic cloud erosion during propagation: Manchester et al. J Geophys Res Space Phys 119(7):5449-5464. https://doi.org/10.1002/2014JA019882

Mandrini CH, Pohjolainen S, Dasso S, Green LM, Démoulin P, van Driel-Gesztelyi L, Copperwheat C, Foley C (2005) Interplanetary flux rope ejected from an xray bright point. The smallest magnetic cloud source-region ever observed. Astron Astrophys 434:725-740. https://doi.org/10.1051/0004-6361:20041079 
Manoharan PK, Maia D, Johri A, Induja MS (2016) Interplanetary consequences of coronal mass ejection events occurred during 18-25 June 2015. 504:59 http://adsabs.harvard.edu/abs/2016ASPC..504...59M

Maričić D, Vršnak B, Stanger AL, Veronig A (2004) Coronal Mass Ejection of 15 May 2001: I. Evolution of morphological features of the eruption. Sol Phys 225(2):337-353. https://doi.org/10.1007/s11207-004-3748-1

Maričić D, Vršnak B, Stanger AL, Veronig AM, Temmer M, Roša D (2007) Acceleration phase of coronal mass ejections: II. Synchronization of the energy release in the associated flare. Sol Phys 241(1):99-112. https://doi. org/10.1007/s11207-007-0291-X

Marubashi K, Akiyama S, Yashiro S, Gopalswamy N, Cho K-S, Park Y-D (2015) Geometrical relationship between interplanetary flux ropes and their solar sources. Textbackslashsolphys 290(5):1371-1397. https://doi.org/10.1007/s112 07-015-0681-4

Marubashi K, Cho K-S, Ishibashi H (2017) Interplanetary Magnetic flux ropes as agents connecting solar eruptions and geomagnetic activities. Sol Phys 292: 189. https://doi.org/10.1007/s11207-017-1204-2

Marubashi K, Cho K-S, Kim R-S, Kim S, Park S-H, Ishibashi H (2016) The 17 March 2015 storm: the associated magnetic flux rope structure and the storm development. Earth Planets Space 68:173. https://doi.org/10.1186/s40623-01 6-0551-9

Marubashi K, Lepping RP (2007) Long-duration magnetic clouds: a comparison of analyses using torus- and cylinder-shaped flux rope models. Ann Geophys 25(11):2453-2477. https://doi.org/10.5194/angeo-25-2453-2007

Mason GM, Desai MI, Cohen CMS, Mewaldt RA, Stone EC, Dwyer JR (2006) The role of interplanetary scattering in western hemisphere large solar energetic particle events. Astrophys J Lett 647(1):L65

Mason GM, Desai MI, Mazur JE, Dwyer JR (2005) Energetic particles accelerated by shocks in the heliosphere: what is the source material? In: AIP Conference Proceedings, vol 781. American Institute of Physics, pp 219-226

Mason GM, Li G, Cohen CMS, Desai MI, Haggerty DK, Leske RA, Mewaldt RA, Zank GP (2012) Interplanetary propagation of solar energetic particle heavy ions observed at $1 \mathrm{AU}$ and the role of energy scaling. Astrophys J 761(2):104

Mason GM, Mazur JE, Dwyer JR (1999) 3He enhancements in large solar energetic particle events. Astrophys J Lett 525(2):L133

Mason GM, Mazur JE, Dwyer JR, Jokipii JR, Gold RE, Krimigis SM (2004) Abundances of heavy and ultraheavy ions in 3He-rich solar flares. Astrophys 606(1):555

Mason JP, Woods TN, Webb DF, Thompson BJ, Colaninno RC, Vourlidas A (2016) Relationship of EUV Irradiance coronal dimming slope and depth to coronal mass ejection speed and mass. Astrophys J 830:20. https://doi.org/10.3847/ 0004-637X/830/1/20

Mays ML, Taktakishvili A, Pulkkinen A, MacNeice PJ, Rastätter L, Odstrcil D, Jian LK et al (2015a) Ensemble modeling of CMEs using the WSA-ENLIL+Cone model. Sol Phys 290(6):1775-1814. https://doi.org/10.1007/s11207-015-0692-1

Mays ML, Thompson BJ, Jian LK, Colaninno RC, Odstrcil D, Möstl C, Temmer M et al (2015) Propagation of the 7 January 2014 CME and Resulting geomagnetic non-event. Astrophys J 812:145. https://doi.org/10.1088/0004-637X/812/2/145

Mazur JE, Mason GM, Dwyer JR, Giacalone J, Jokipii JR, Stone EC (2000) The mixing of interplanetary magnetic field lines: a significant transport effect in studies of the energy spectra of impulsive flares. AIP Conf Proc 528(1):47-54. https://doi.org/10.1063/1.1324280

Mazur JE, Mason GM, Looper MD, Leske RA, Mewaldt RA (1999) Charge states of solar energetic particles using the geomagnetic cutoff technique: SAMPEX measurements in the 6 November 1997 solar particle event. Geophys Res Lett 26(2):173-176

McAllister $\mathrm{AH}$, Hundhausen AJ (1996) The relation of $\mathrm{YOHKOH}$ coronal arcades events to coronal streamers and CMEs, vol 95, p 171 http://adsabs.harvard. edu/abs/1996ASPC...95..171M

McCauley PI, Su YN, Schanche N, Evans KE, Su C, McKillop S, Reeves KK (2015) Prominence and filament eruptions observed by the solar dynamics observatory: statistical properties, kinematics, and online catalog. Sol Phys 290:1703-1740. https://doi.org/10.1007/s11207-015-0699-7

McComas DJ, Alexander N, Angold N, Bale S, Beebe C, Birdwell B, Boyle M et al (2016) Integrated Science Investigation of the Sun (ISIS): Design of the energetic particle investigation. SSr 204(1-4):187-256

McGregor SL, Hughes WJ, Arge CN, Owens MJ (2008) Analysis of the magnetic field discontinuity at the potential field source surface and schatten current sheet interface in the Wang-Sheeley-Arge model. J Geophys Res (Space Physics) 113:A08112. https://doi.org/10.1029/2007JA012330
Meng X, van der Holst B, Tóth G, Gombosi Tl (2015) Alfvén wave solar model (AWSOM): proton temperature anisotropy and solar wind acceleration. Mon Notices R Astronomical Soc 454(4):3697-3709. https://doi.org/10.1093/mnras/ stv2249

Merkin VG, Kondrashov D, Ghil M, Anderson BJ (2016a) Data assimilation of lowaltitude magnetic perturbations into a global magnetosphere model: assimilation of magnetic perturbations. Space Weather 14(2):165-184. https:// doi.org/10.1002/2015SW001330

Merkin VG, Lionello R, Lyon JG, Linker J, Török T, Downs C (2016b) Coupling of coronal and heliospheric magnetohydrodynamic models: solution comparisons and verification. Astrophys J 831(1):23. https://doi.org/10.3847/ 0004-637X/831/1/23

Merkin VG, Lyon JG, McGregor SL, Pahud DM (2011) Disruption of a heliospheric current sheet fold: HCS fold. Geophys Res Lett 38(14):n/a. https://doi.org/10.1 029/2011GL047822

Mewaldt RA, Cohen CMS, Cook WR, Cummings AC, Davis AJ, Geier S, Kecman B et al (2008) The low-energy telescope (LET) and SEP central electronics for the STEREO mission. Space Sci Rev 136(1-4):285-362

Mewaldt RA, Cohen CMS, Labrador AW, Leske RA, Mason GM, Desai MI, Looper MD, Mazur JE, Selesnick RS, Haggerty DK (2005) Proton, helium, and electron spectra during the large solar particle events of October-November 2003. J Geophys Res Space Phys 110(A9)

Mewaldt RA, Cohen CMS, Leske RA, Christian ER, Cummings AC, Stone EC, von Rosenvinge TT, Wiedenbeck ME (2002) Fractionation of solar energetic particles and solar wind according to first ionization potential. Adv Space Res 30(1):79-84

Mewaldt RA, Looper MD, Cohen CMS, Haggerty DK, Labrador AW, Leske RA, Mason GM, Mazur JE, von Rosenvinge TT (2012a) Energy spectra, composition, and other properties of ground-level events during solar cycle 23. Space Sci Rev 171:97-120. https://doi.org/10.1007/s11214-012-9884-2

Mewaldt RA, Mason GM, CMS C (2012b) The dependence of solar energetic particle fluences on suprathermal seed-particle densities. 1500:128-133. https://doi.org/10.1063/1.4768755

Mewaldt RA, Mason GM, Cohen CMS, Gómez-Herrero R, Haggerty DK, Leske RA, Wiedenbeck ME (2012c) Evolution of Suprathermal seed particle and solar energetic particle abundances. 1436:206-211. https://doi.org/10.1063/1.4723 609

Michalek G, Gopalswamy N, Yashiro S (2019) On the coronal mass ejection detection rate during solar cycles 23 and 24. Astrophys J 880:51. https://doi. org/10.3847/1538-4357/ab26a7

Mierla M, Inhester B, Antunes A, Boursier Y, Byrne JP, Colaninno R, Davila J et al (2010) On the 3-D reconstruction of coronal mass ejections using coronagraph data. Ann Geophys 28:203-215. https://doi.org/10.5194/angeo-2 8-203-2010

Miklenic CH, Veronig AM, Vršnak B (2009) Temporal comparison of nonthermal flare emission and magnetic-flux change rates. Astron Astrophys 499(3):893904. https://doi.org/10.1051/0004-6361/200810947

Mishra W, Srivastava N (2014) Morphological and kinematic evolution of three interacting coronal mass ejections of 2011 February 13-15. Astrophys J 794(1):64. https://doi.org/10.1088/0004-637X/794/1/64

Mishra W, Srivastava N, Chakrabarty D (2014) Evolution and consequences of interacting CMEs of 2012 November 9-10 using STEREO/SECCHI and in situ observations. Sol Phys in press

Mishra W, Srivastava N, Singh T (2015) Kinematics of interacting CMEs of 25 and 28 September 2012: interacting CMEs. J Geophys Res Space Phys 120(12): 10,221-10,236. https://doi.org/10.1002/2015JA021415

Mishra W, Wang Y (2018) Modeling the thermodynamic evolution of coronal mass ejections using their kinematics. Astrophys J 865(1):50. https://doi.org/1 $0.3847 / 1538-4357 / a a d b 9 b$

Mishra W, Wang Y, Srivastava N (2016) On understanding the nature of collisions of coronal mass ejections observed by STEREO. Astrophys J 831(1):99. https:// doi.org/10.3847/0004-637X/831/1/99

Mishra W, Wang Y, Srivastava N, Shen C (2017) Assessing the nature of collisions of coronal mass ejections in the inner heliosphere. Astrophys J Suppl Ser 232(1):5. https://doi.org/10.3847/1538-4365/aa8139

Mishra W, Wang Y, Teriaca L, Zhang J, Chi Y (2020) Probing the thermodynamic state of a coronal mass ejection (CME) Up to 1 AU. Front Astron Space Sci 7: 1. https://doi.org/10.3389/fspas.2020.00001

Miteva R, Klein K-L, Kienreich I, Temmer M, Veronig A, Malandraki OE (2014) Solar energetic particles and associated EIT disturbances in solar cycle 23. Sol Phys 289(7):2601-2631 
Mitra PK, Joshi B, Prasad A, Veronig AM, Bhattacharyya R (2018) Successive flux rope eruptions from \$lupdelta\$-sunspots region of NOAA 12673 and associated X-class eruptive flares on 2017 September 6. Astrophys J 869(1):69. https://doi.org/10.3847/1538-4357/aaed26

Miyoshi Y, Kataoka R, Kasahara Y, Kumamoto A, Nagai T, Thomsen MF (2013) High-speed solar wind with southward interplanetary magnetic field causes relativistic electron flux enhancement of the outer radiation belt via enhanced condition of whistler waves. Grl 40(17):4520-4525. https://doi. org/10.1002/grl.50916

Möbius E, Popecki M, Klecker B, Kistler LM, Bogdanov A, Galvin AB, Heirtzler D et al (1999) Energy dependence of the ionic charge state distribution during the November 1997 solar energetic particle event. Geophys Res Lett 26(2):145-148

Mohamed AA, Gopalswamy N, Yashiro S, Akiyama S, Mäkelä P, Xie H, Jung H (2012) The relation between coronal holes and coronal mass ejections during the rise, maximum, and declining phases of solar cycle 23: $\mathrm{CME}-\mathrm{CH}$ interaction. J Geophys Res Space Phys 117(A1). https://doi.org/10.1029/2011 JA016589

Mondal S, Oberoi D, Vourlidas A (2020) Estimation of the physical parameters of a cme at high coronal heights using low-frequency radio observations. Astrophys J 893:28. https://doi.org/10.3847/1538-4357/ab7fab

Moon Y-J, Choe GS, Wang H, Park YD, Gopalswamy N, Guo Y, Yashiro S (2002) A statistical study of two classes of coronal mass ejections. Astrophys J 581(1): 694-702. https://doi.org/10.1086/344088

Moraitis K, Sun X, Pariat É, Linan L (2019) Magnetic helicity and eruptivity in active region 12673. Astron Astrophys 628:A50. https://doi.org/10.1051/0004$6361 / 201935870$

Moraitis K, Tziotziou K, Georgoulis MK, Archontis V (2014) Validation and benchmarking of a practical free magnetic energy and relative magnetic helicity budget calculation in solar magnetic structures. Sol Phys 289:44534480. https://doi.org/10.1007/s11207-014-0590-y

Moschou S-P, Pierrard V, Keppens R, Pomoell J (2017) Interfacing MHD single fluid and kinetic exospheric solar wind models and comparing their energetics. Sol Phys 292(9):139. https://doi.org/10.1007/s11207-017-1164-6

Möstl C, Amerstorfer T, Palmerio E, Isavnin A, Farrugia CJ, Lowder C, Winslow RM, Donnerer JM, Kilpua EK, Boakes PD (2018) Forward modeling of coronal mass ejection flux ropes in the inner heliosphere with 3DCORE. Space Weather 16(3):216-229. https://doi.org/10.1002/2017SW001735

Möstl C, Amla K, Hall JR, Liewer PC, De Jong EM, Colaninno RC, Veronig AM et al (2014) Connecting speeds, directions and arrival times of 22 coronal mass ejections from the Sun to $1 \mathrm{AU}$. Astrophys J 787:119. https://doi.org/10.1088/ 0004-637X/787/2/119

Möstl C, Farrugia CJ, Kilpua EKJ, Jian LK, Liu Y, Eastwood JP, Harrison RA et al (2012) Multi-point shock and flux rope analysis of multiple interplanetary coronal mass ejections around 2010 August 1 in the inner heliosphere. Astrophys J 758(1):10. https://doi.org/10.1088/0004-637X/758/1/10

Möstl C, Rollett T, Frahm RA, Liu YD, Long DM, Colaninno RC, Martin A. Reiss, et al. (2015) Strong Coronal channelling and interplanetary evolution of a solar storm up to Earth and Mars. Nat Commun 6:7135. https://doi.org/10.1038/ ncomms8135

Muhr N, Veronig AM, Kienreich IW, Vršnak B, Temmer M, Bein BM (2014) Statistical analysis of large-scale EUV waves observed by STEREO/EUVI. Sol Phys 289(12):4563-4588. https://doi.org/10.1007/s11207-014-0594-7

Müller D, Cyr OCS, Zouganelis I, Gilbert HR, Marsden R, Nieves-Chinchilla T, Antonucci E et al (2020) The Solar Orbiter mission. Science overview. Astron Astrophys 642:A1. https://doi.org/10.1051/0004-6361/202038467

Mulligan T, Russell CT (2001) Multispacecraft modeling of the flux rope structure of interplanetary coronal mass ejections: cylindrically symmetric versus nonsymmetric topologies. J Geophys Res Space Phys 106(A6):10581-10596. https://doi.org/10.1029/2000JA900170

Munro RH, Gosling JT, Hildner E, MacQueen RM, Poland Al, Ross CL (1979) The association of coronal mass ejection transients with other forms of solar activity. Sol Phys 61(1):201-215. https://doi.org/10.1007/BF00155456

Nakagawa Y, Nozawa S, Shinbori A (2019) Relationship between the low-latitude coronal hole area, solar wind velocity, and geomagnetic activity during solar cycles 23 and 24. Earth Planets Space 71:24. https://doi.org/10.1186/s40623019-1005-y

Napoletano G, Forte R, Del Moro D, Pietropaolo E, Giovannelli L, Berrilli F (2018) A probabilistic approach to the drag-based model. 335:304-306. https://doi. org/10.1017/S1743921317008122

Neupert WM, Thompson BJ, Gurman JB, Plunkett SP (2001) Eruption and acceleration of flare-associated coronal mass ejection loops in the low corona. J Geophys Res Space Phys 106(A11):25215-25225. https://doi.org/1 0.1029/2000JA004012

$\mathrm{Ng}$ CK, Reames DV, Tylka AJ (1999) Effect of proton-amplified waves on the evolution of solar energetic particle composition in gradual events. Geophys Res Lett 26(14):2145-2148

Ng CK, Reames DV, Tylka AJ (2003) Modeling shock-accelerated solar energetic particles coupled to interplanetary Alfvén waves. Astrophys J 591(1):461

Ng CK, Reames DV, Tylka AJ (2012) Solar energetic particles: shock acceleration and transport through self-amplified waves. In: AIP Conference Proceedings, vol 1436. American Institute of Physics, pp 212-218

Nieves-Chinchilla T, Jian LK, Balmaceda L, Vourlidas A, dos Santos LFG, Szabo A (2019) Unraveling the internal magnetic field structure of the Earth-directed interplanetary coronal mass ejections during 1995 - 2015. Sol Phys 294:89. https://doi.org/10.1007/s11207-019-1477-8

Nieves-Chinchilla T, Linton MG, Hidalgo MA, Vourlidas A (2018b) Ellipticcylindrical analytical flux rope model for magnetic clouds. Astrophys J 861 (2): 139. https://doi.org/10.3847/1538-4357/aac951

Nieves-Chinchilla T, Linton MG, Hidalgo MA, Vourlidas A, Savani NP, Szabo A, Farrugia C, Yu W (2016) A circular-cylindrical flux-rope analytical model for magnetic clouds. Astrophys J 823(1):27. https://doi.org/10.3847/0004-637X/ $823 / 1 / 27$

Nieves-Chinchilla T, Szabo A, Korreck KE, Alzate N, Balmaceda LA, Lavraud B, Paulson K et al (2020) Analysis of the Internal structure of the streamer blowout observed by the Parker Solar Probe during the first solar encounter. Astrophys J Suppl Ser 246:63. https://doi.org/10.3847/1538-4365/ab61f5

Nieves-Chinchilla T, Vourlidas A, Raymond JC, Linton MG, Al-haddad N, Savani NP, Szabo A, Hidalgo MA (2018a) Understanding the internal magnetic field configurations of ICMEs using more than 20 years of wind observations. Sol Phys 293:25. https://doi.org/10.1007/s11207-018-1247-z

Nindos A, Andrews MD (2004) The association of big flares and coronal mass ejections: what is the role of magnetic helicity? Astrophys J Lett 616:L175L178. https://doi.org/10.1086/426861

Nindos A, Kontar EP, Oberoi D (2019) Solar physics with the square kilometre array. Adv Space Res 63:1404-1424. https://doi.org/10.1016/j.asr.2018.10.023

Nindos A, Patsourakos S, Vourlidas A, Cheng X, Zhang J (2020) When do solar erupting hot magnetic flux ropes form? Astron Astrophys 642:A109. https:// doi.org/10.1051/0004-6361/202038832

Nindos A, Patsourakos S, Vourlidas A, Tagikas C (2015) How common are hot magnetic flux ropes in the low solar corona? A statistical study of EUV observations. Astrophys J 808:117. https://doi.org/10.1088/0004-637X/ $808 / 2 / 117$

Nindos A, Patsourakos S, Wiegelmann T (2012) On the Role of the background overlying magnetic field in solar eruptions. Astrophys J Lett 748:L6. https:// doi.org/10.1088/2041-8205/748/1/L6

Nishimura N, Marubashi K, Tokumaru M (2019) Comparison of cylindrical interplanetary flux-rope model fitting with different boundary pitch-angle treatments. Sol Phys 294(4):49. https://doi.org/10.1007/s11207-019-1435-5

Nitta NV, Liu W, Gopalswamy N, Yashiro S (2014) The relation between largescale coronal propagating fronts and type II radio bursts. Sol Phys 289:45894606. https://doi.org/10.1007/s11207-014-0602-y

Nitta NV, Mulligan T (2017) Earth-affecting coronal mass ejections without obvious low coronal signatures. Sol Phys 292(9):125. https://doi.org/10.1007/ s11207-017-1147-7

Nitta NV, Schrijver CJ, Title AM, Liu W (2013) Large-scale coronal propagating fronts in solar eruptions as observed by the atmospheric imaging assembly on board the solar dynamics observatory-an ensemble studY. Astrophys J 776(1):58. https://doi.org/10.1088/0004-637X/776/1/58

Nolte JT, Krieger AS, Timothy AF, Gold RE, Roelof EC, Vaiana G, Lazarus AJ, Sullivan JD, McIntosh PS (1976) Coronal holes as sources of solar wind. Sol Phys 46:303-322. https://doi.org/10.1007/BF00149859

Odstrčil D, Dryer M, Smith Z (1996) Propagation of an Interplanetary shock along the heliospheric plasma sheet. J Geophys Res 101:19973-19986. https://doi. org/10.1029/96JA00479

Odstrčil D, Pizzo VJ (1999a) Three-dimensional propagation of coronal mass ejections (CMEs) in a structured solar wind flow: 1. CME launched within the streamer belt. J Geophys Res Space Phys 104(A1):483-492. https://doi.org/1 0.1029/1998JA900019

Odstrčil D, Pizzo VJ (1999b) Three-dimensional propagation of coronal mass ejections (CMEs) in a structured solar wind flow: 2. CME launched adjacent to the streamer belt. J Geophys Res Space Phys 104(A1):493-503. https://doi. org/10.1029/1998JA900038 
Odstrcil D, Pizzo VJ, Arge CN (2005) Propagation of the 12 May 1997 interplanetary coronal mass ejection in evolving solar wind structures. $J$ Geophys Res 110(A2):A02106. https://doi.org/10.1029/2004JA010745

Odstrcil D, Riley P, Zhao XP (2004) Numerical simulation of the 12 May 1997 interplanetary CME event: brief report. J Geophys Res Space Phys 109(A2) https://doi.org/10.1029/2003JA010135

Oetliker M, Klecker B, Hovestadt D, Mason GM, Mazur JE, Leske RA, Mewaldt RA, Blake JB, Looper MD (1997) The ionic charge of solar energetic particles with energies of 0.3-70 MeV per nucleon. Astrophys J 477(1):495

Ogilvie KW, Chornay DJ, Fritzenreiter RJ, Hunsaker F, Keller J, Lobell J, Miller G et al (1995) SWE, A comprehensive plasma instrument for the wind spacecraft. Space Sci Rev 71:55-77. https://doi.org/10.1007/BF00751326

Oliveros M, Carlos J, Raftery CL, Bain HM, Liu Y, Krupar V, Bale S, Krucker S (2012) The 2010 August 1 type ii burst: a CME-CME interaction and its radio and white-light manifestations. Astrophys J 748(1):66. https://doi.org/10.1088/ 0004-637X/748/1/66

Olmedo O, Zhang J (2010) Partial torus instability. Astrophys J 718:433-440. https://doi.org/10.1088/0004-637X/718/1/433

Olmedo O, Zhang J, Wechsler H, Poland A, Borne K (2008) Automatic detection and tracking of coronal mass ejections in coronagraph time series. Sol Phys 248:485-499. https://doi.org/10.1007/s11207-007-9104-5

Ontiveros V, Vourlidas A (2009) Quantitative measurements of coronal mass ejection-driven shocks from LASCO observations. Astrophys J 693:267-275. https://doi.org/10.1088/0004-637X/693/1/267

Opitz A, Karrer R, Wurz P, Galvin AB, Bochsler P, Blush LM, Daoudi H et al (2009) Temporal evolution of the solar wind bulk velocity at solar minimum by correlating the STEREO A and B PLASTIC measurements. Sol Phys 256(1-2): 365-377. https://doi.org/10.1007/s11207-008-9304-7

Oran R, Landi E, van der Holst B, Lepri ST, Vásquez AM, Nuevo FA, Frazin R, Manchester W, Sokolov I, Gombosi TI (2015) A steady-state picture of solar wind acceleration and charge state composition derived from a global wavedriven MHD model. Astrophys J 806(1):55. https://doi.org/10.1088/0004-637X $806 / 1 / 55$

Owens MJ, Démoulin P, Savani NP, Lavraud B, Ruffenach A (2012) Implications of non-cylindrical flux ropes for magnetic cloud reconstruction techniques and the interpretation of double flux rope events. Sol Phys 278(2):435-446. https://doi.org/10.1007/s11207-012-9939-2

Owens MJ, Forsyth RJ (2013) The Heliospheric Magnetic Field. Living Reviews in Solar Physics 10(1):5. https://doi.org/10.12942//rsp-2013-5

Owens MJ, Riley P, Lang M, Lockwood M (2019) Near-Earth solar wind forecasting using corotation from L5: the error introduced by heliographic latitude offset. Space Weather 17(7):1 105-1113. https://doi.org/10.1029/2019SW002204

Paassilta M, Raukunen O, Vainio R, Valtonen E, Papaioannou A, Siipola R, Riihonen E et al (2017) Catalogue of 55-80 MeV solar proton events extending through solar cycles 23 and 24. J Space Weather Space Climate 7:A14. https://doi.org/10.1051/swsc/2017013

Pacheco D, Agueda N, Aran A, Heber B, Lario D (2019) Full inversion of solar relativistic electron events measured by the helios spacecraft. Astron Astrophys 624:A3. https://doi.org/10.1051/0004-6361/201834520

Pagano P, Bemporad A, Mackay DH (2015) Future capabilities of CME polarimetric 3D reconstructions with the METIS instrument: a numerical test. Astron Astrophys 582:A72. https://doi.org/10.1051/0004-6361/201425462

Pagano P, Mackay DH, Yardley SL (2019) A prospective new diagnostic technique for distinguishing eruptive and noneruptive active regions. Astrophys J 883: 112. https://doi.org/10.3847/1538-4357/ab3e42

Pal S, Nandy D, Srivastava N, Gopalswamy N, Panda S (2018) Dependence of coronal mass ejection properties on their solar source active region characteristics and associated flare reconnection flux. Astrophys J 865:4. https://doi.org/10.3847/1538-4357/aada10

Pallavicini R, Serio S, Vaiana GS (1977) A survey of soft x-ray limb flare images the relation between their structure in the corona and other physical parameters. Astrophys J 216:108-122. https://doi.org/10.1086/155452

Pallocchia G, Laurenza M, Consolini G (2017) On Weibull's spectrum of nonrelativistic energetic particles at IP shocks: observations and theoretical interpretation. Astrophys J 837(2):158

Palmerio E, Kilpua EKJ, James AW, Green LM, Pomoell J, Isavnin A, Valori G (2017) Determining the intrinsic CME Flux rope type using remote-sensing solar disk observations. Sol Phys 292:39. https://doi.org/10.1007/s11207-017-1063-x

Palmerio E, Kilpua EKJ, Möstl C, Bothmer V, James AW, Green LM, Isavnin A, Davies JA, Harrison RA (2018) Coronal magnetic structure of earthbound
CMEs and in situ comparison. Space Weather 16:442-460. https://doi.org/10.1 002/2017SW001767

Palmerio E, Scolini C, Barnes D, Magdalenić J, West MJ, Zhukov AN, Rodriguez L et al (2019) Multipoint study of successive coronal mass ejections driving moderate disturbances at $1 \mathrm{Au}$. Astrophys J 878:37. https://doi.org/10.3847/1 538-4357/ab1850

Paouris E, Mavromichalaki H (2017) Effective acceleration model for the arrival time of interplanetary shocks driven by coronal mass ejections. Sol Phys 292(12):180. https://doi.org/10.1007/s11207-017-1212-2

Parenti S (2014) Solar prominences: observations. Living Rev Sol Phys 11:1. https://doi.org/10.12942//rsp-2014-1

Pariat E, Leake JE, Valori G, Linton MG, Zuccarello FP, Dalmasse K (2017) Relative magnetic helicity as a diagnostic of solar eruptivity. Astron Astrophys 601: A125. https://doi.org/10.1051/0004-6361/201630043

Park J, Innes DE, Bucik R, Moon Y-J (2013) The source regions of solar energetic particles detected by widely separated spacecraft. Astrophys J 779(2):184

Park S-H, Lee J, Choe GS, Chae J, Jeong H, Guo Y, Jing J, Wang H (2008) The variation of relative magnetic helicity around major flares. Astrophys J 686: 1397-1403. https://doi.org/10.1086/591117

Park, Sung-Hong, K. D. Leka, Kanya Kusano, Jesse Andries, Graham Barnes, Suzy Bingham, D. Shaun Bloomfield, et al. 2020. A comparison of flare forecasting methods. IV. Evaluating consecutive-day forecasting patterns." ArXiv:2001. 02808 [Astro-Ph], January. http://arxiv.org/abs/2001.02808.

Parker EN (1965) The passage of energetic charged particles through interplanetary space. Planetary Space Sci 13(1):9-49

Patsourakos S, Georgoulis MK (2017) A helicity-based method to infer the CME magnetic field magnitude in sun and geospace: generalization and extension to Sun-like and M-dwarf stars and implications for exoplanet habitability. Sol Phys 292:89. https://doi.org/10.1007/s11207-017-1124-1

Patsourakos S, Georgoulis MK, Vourlidas A, Nindos A, Sarris T, Anagnostopoulos G, Anastasiadis A, Chintzoglou G, Daglis IA, Gontikakis C, Hatzigeorgiu N, Iliopoulos AC, Katsavrias C, Kouloumvakos A, Moraitis K, Nieves-Chinchilla T, Pavlos G, Sarafopoulos D, Syntelis P, Tsironis C, Tziotziou K, Vogiatzis II, Balasis G, Georgiou M, Karakatsanis LP, Malandraki OE, Papadimitriou C, Odstrčil D, Pavlos EG, Podlachikova O, I. Sand berg, et al. (2016) The major geoeffective solar eruptions of 2012 March 7: comprehensive Sun-to-Earth analysis. Astrophys J 817(1):14. https://doi.org/10.3847/0004-637X/817/1/14

Patsourakos S, Vourlidas A (2009) 'Extreme ultraviolet waves' are waves: first quadrature observations of an extreme ultraviolet wave from STEREO. Astrophys J Lett 700:L182-L186. https://doi.org/10.1088/0004-637X/700/2/L182

Patsourakos S, Vourlidas A, Kliem B (2010) Toward understanding the early stages of an impulsively accelerated coronal mass ejection. SECCHI observations. Astron Astrophys 522:A100. https://doi.org/10.1051/0004-6361/200913599

Patsourakos S, Vourlidas A, Stenborg G (2013) Direct evidence for a fast coronal mass ejection driven by the prior formation and subsequent destabilization of a magnetic flux rope. Astrophys J 764:125. https://doi.org/10.1088/0004-63 $7 X / 764 / 2 / 125$

Patsourakos S, Vourlidas A, Török T, Kliem B, Antiochos SK, Archontis V, Aulanier G et al (2020) Decoding the Pre-eruptive magnetic field configurations of coronal mass ejections. ArXiv E-Prints 2010:arXiv:2010.10186 http://adsabs.ha rvard.edu/abs/2020arXiv201010186P

Pesnell W, Dean B, Thompson J, Chamberlin PC (2012) The Solar Dynamics Observatory (SDO). Sol Phys 275:3-15. https://doi.org/10.1007/s11207-0119841-3

Petrie GJD (2015) On the enhanced coronal mass ejection detection rate since the solar cycle 23 polar field reversal. Astrophys J 812:74. https://doi.org/10.1 088/0004-637X/812/1/74

Petrovay K (2010) Solar cycle prediction. Living Rev Sol Phys 7:6. https://doi.org/1 0.12942/Irsp-2010-6

Pevtsov AA, Balasubramaniam KS, Rogers JW (2003) Chirality of chromospheric filaments. Astrophys J 595:500-505. https://doi.org/10.1086/377339

Pevtsov AA, Berger MA, Nindos A, Norton AA, van Driel-Gesztelyi L (2014) Magnetic helicity, tilt, and twist. Space Sci Rev 186:285-324. https://doi.org/1 0.1007/s11214-014-0082-2

Piantschitsch I, Vršnak B, HansImeier A, Lemmerer B, Veronig A, Hernandez-Perez A, Čalogović J (2018a) Numerical simulation of coronal waves interacting with coronal holes. II. Dependence on Alfvén speed inside the coronal hole. Astrophys J 857(2):130. https://doi.org/10.3847/1538-4357/aab709

Piantschitsch I, Vršnak B, Hanslmeier A, Lemmerer B, Veronig A, Hernandez-Perez A, Čalogović J (2018b) Numerical simulation of coronal waves interacting 
with coronal holes. III. Dependence on initial amplitude of the incoming wave. Astrophys J 860(1):24. https://doi.org/10.3847/1538-4357/aabe7f

Piantschitsch I, Vršnak B, HansImeier A, Lemmerer B, Veronig A, Hernandez-Perez A, Čalogović J, Žic T (2017) A numerical simulation of coronal waves interacting with coronal holes. I. Basic features. Astrophys J 850(1):88. https:// doi.org/10.3847/1538-4357/aa8cc9

Pizzo V (1978) A three-deminsional model of corotating streams in the solar wind 1. Theoretical foundations. 83(A12):5563-5572. https://doi.org/10.1029/ JA083iA12p05563.\&nbsp

Plotnikov I, Rouillard AP, Share GH (2017) The magnetic connectivity of coronal shocks from behind-the-limb flares to the visible solar surface during $y$-ray events. Astron Astrophys 608:A43

Pomoell J, Lumme E, Kilpua E (2019) Time-dependent data-driven modeling of active region evolution using energy-optimized photospheric electric fields. Sol Phys 294:41. https://doi.org/10.1007/s11207-019-1430-x

Pomoell J, Poedts S (2018) EUHFORIA: European Heliospheric Forecasting Information Asset. J Space Weather Space Climate 8:A35. https://doi.org/10.1 051/swsc/2018020

Poomvises W, Zhang J, Olmedo O (2010) Coronal mass ejection propagation and expansion in three-dimensional space in the heliosphere based on Stereo/ SECCHI observations. Astrophys J Lett 717:L159-L163. https://doi.org/10.1 088/2041-8205/717/2/L159

Price DJ, Pomoell J, Lumme E, Kilpua EKJ (2019) Time-Dependent data-driven coronal simulations of AR 12673 from emergence to eruption. Astron Astrophys 628:A114. https://doi.org/10.1051/0004-6361/201935535

Priest ER, Forbes TG (2002) The magnetic nature of solar flares. Astron Astrophys Rev 10(4):313-377. https://doi.org/10.1007/s001590100013

Pulkkinen T (2007) Space weather: terrestrial perspective. Living Rev Sol Phys 4:1. https://doi.org/10.12942//rsp-2007-1

Qiu J, Hu Q, Howard TA, Yurchyshyn VB (2007) On the magnetic flux budget in low-corona magnetic reconnection and interplanetary coronal mass ejections. Astrophys J 659:758-772. https://doi.org/10.1086/512060

Qiu J, Yurchyshyn VB (2005) Magnetic reconnection flux and coronal mass ejection velocity. Astrophys J 634(1):L121-L124. https://doi.org/10.1086/498716

Reames DV (1990) Acceleration of Energetic particles by shock waves from large solar flares. Astrophys J 358:L63-L67

Reames DV (1999) Particle acceleration at the Sun and in the heliosphere. Space Sci Rev 90(3-4):413-491

Reames DV (2013) The two sources of solar energetic particles. Space Sci Rev 175(1-4):53-92

Reames DV (2014) Element Abundances in Solar Energetic Particles and the Solar Corona. 289(3):977-993. https://doi.org/10.1007/s11207-013-0350-4

Reames DV (2015) What are the sources of solar energetic particles? Element abundances and source plasma temperatures. Space Sci Rev 194(1-4):303-327

Reames DV (2016) Temperature of the source plasma in gradual solar energetic particle events. Sol Phys 291(3):911-930

Reames DV (2018) Abundances, ionization states, temperatures, and FIP in Solar energetic particles. Space Sci Rev 214(3):61

Reames DV (2020) Four distinct pathways to the element abundances in solar energetic particles. Space Sci Rev 216(2):1-29

Reeves GD, McAdams KL, Friedel RHW, O'Brien TP (2003) Acceleration and loss of relativistic electrons during geomagnetic storms. Grl 30(10):1529. https://doi. org/10.1029/2002GL016513

Reiner MJ, Kaiser ML, Bougeret J-L (2007) Coronal and interplanetary propagation of CME/shocks from radio, in situ and white-light observations. Astrophys J 663:1369-1385. https://doi.org/10.1086/518683

Reiss MA, Hofmeister SJ, De Visscher R, Temmer M, Veronig AM, Delouille V, Mampaey B, Ahammer H (2015) Improvements on coronal hole detection in SDO/AIA images using supervised classification. J Space Weather Space Climate 5:A23. https://doi.org/10.1051/swsc/2015025

Reiss MA, Temmer M, Veronig AM, Nikolic L, Vennerstrom S, Schöngassner F, Hofmeister SJ (2016) Verification of high-speed solar wind stream forecasts using operational solar wind models. Space Weather 14(7):495-510. https:// doi.org/10.1002/2016SW001390

Richardson IG (2018) Solar wind stream interaction regions throughout the heliosphere. Living Rev Sol Phys 15:1. https://doi.org/10.1007/s41116-017$0011-z$

Richardson IG, Cane HV (2010) Near-Earth interplanetary coronal mass ejections during solar cycle 23 (1996 - 2009): Catalog and summary of properties. Sol Phys 264:189-237. https://doi.org/10.1007/s11207-010-9568-6
Richardson IG, Cane HV (2011) Geoeffectiveness (Dst and Kp) of Interplanetary coronal mass ejections during 1995-2009 and implications for storm forecasting. Space Weather 9:7005. https://doi.org/10.1029/2011SW000670

Richardson IG, Cane HV, Von Rosenvinge TT (1991) Prompt arrival of solar energetic particles from far eastern events: the role of large-scale interplanetary magnetic field structure. J Geophys Res Space Phys 96(A5): 7853-7860

Richardson IG, Cliver EW, Cane HV (2001) Sources of Geomagnetic Storms for Solar Minimum and Maximum Conditions during 1972-2000. Geophys Res Lett 28:2569-2572. https://doi.org/10.1029/2001GL013052

Richardson IG, von Rosenvinge TT, Cane HV, Christian ER, Cohen CMS, Labrador AW, Leske RA, Mewaldt RA, Wiedenbeck ME, Stone EC (2014) > $25 \mathrm{MeV}$ proton events observed by the high energy telescopes on the STEREO A and B spacecraft and/or at Earth during the first seven years of the STEREO mission. In: Coronal Magnetometry. Springer, pp 437-485

Riley P, Baker D, Liu YD, Verronen P, Singer H, Güdel M (2018a) Extreme space weather events: from cradle to grave. Space Sci Rev 214:21. https://doi.org/1 0.1007/s11214-017-0456-3

Riley P, Ben-Nun M, Linker JA, Owens MJ, Horbury TS (2017) Forecasting the properties of the solar wind using simple pattern recognition: solar wind prediction. Space Weather 15(3):526-540. https://doi.org/10.1002/2016SW001 589

Riley P, Crooker NU (2004) Kinematic Treatment of coronal mass ejection evolution in the solar wind. Astrophys J 600(2):1035-1042. https://doi.org/1 $0.1086 / 379974$

Riley P, Leila Mays M, Andries J, Amerstorfer T, Biesecker D, Delouille V, Dumbović $M$ et al (2018b) Forecasting the arrival time of coronal mass ejections: analysis of the CCMC CME scoreboard. Space Weather 16:1245-1260. https:// doi.org/10.1029/2018SW001962

Riley P, Linker JA, Lionello R, Mikić Z, Odstrcil D, Hidalgo MA, Cid C et al (2004) Fitting flux ropes to a global MHD solution: a comparison of techniques. J Atmos Sol Terr Phys 66(15-16):1321-1331. https://doi.org/10.1016/j.jastp.2004 03.019

Robbrecht E, Berghmans D (2004) Automated recognition of coronal mass ejections (CMEs) in near-real-time data. Astron Astrophys 425:1097-1106. https://doi.org/10.1051/0004-6361:20041302

Robbrecht E, Berghmans D, Van der Linden RAM (2009a) Automated LASCO CME catalog for solar cycle 23: are CMEs scale invariant? Astrophys J 691:12221234. https://doi.org/10.1088/0004-637X/691/2/1222

Robbrecht E, Patsourakos S, Vourlidas A (2009b) No trace left behind: STEREO observation of a coronal mass ejection without low coronal signatures. Astrophys J 701(1):283-291. https://doi.org/10.1088/0004-637X/701/1/283

Rodríguez-Pacheco J, Wimmer-Schweingruber RF, Mason GM, Ho GC, SánchezPrieto S, Prieto M, Martín C et al (2020) The energetic particle detector. energetic particle instrument suite for the Solar Orbiter mission. Aap 642:A7

Rollett T, Möstl C, Isavnin A, Davies JA, Kubicka M, Amerstorfer UV, Harrison RA (2016) ElEvoHI: a novel CME prediction tool for heliospheric imaging combining an elliptical front with drag-based model fitting. Astrophys J. 824: 131. https://doi.org/10.3847/0004-637X/824/2/131

Romashets E, Vandas M (2013) Modeling irregularities in solar flux ropes. Sol Phys 284(1):235-243. https://doi.org/10.1007/s11207-012-0083-9

Romashets EP, Vandas M (2003) Force-free field inside a toroidal magnetic cloud: toroidal magnetic cloud. Geophys Res Lett 30(20):n/a. https://doi.org/10.102 9/2003GL017692

Rotter T, Veronig AM, Temmer M, Vršnak B (2012) Relation between coronal hole areas on the Sun and the solar wind parameters at 1 AU. Sol Phys 281:793813. https://doi.org/10.1007/s11207-012-0101-y

Rotter T, Veronig AM, Temmer M, Vršnak B (2015) Real-time solar wind prediction based on SDO/AIA coronal hole data. Sol Phys 290(5):1355-1370. https://doi. org/10.1007/s11207-015-0680-5

Rouillard AP (2011) Relating white light and in situ observations of coronal mass ejections: a review. J Atmos Sol Terr Phys 73:1201-1213. https://doi.org/10.1 016/j.jastp.2010.08.015

Rouillard AP, Davies JA, Forsyth RJ, Rees A, Davis CJ, Harrison RA, Lockwood M et al (2008) First imaging of corotating interaction regions using the STEREO spacecraft. Geophys Res Lett 35:L10110. https://doi.org/10.1029/2008GL033 767

Rouillard AP, Plotnikov I, Pinto RF, Tirole M, Lavarra M, Zucca P, Vainio R et al (2016) Deriving the properties of coronal pressure fronts in 3D: application to the 2012 May 17 ground level enhancement. Astrophys J 833(1):45 
Roussev II, Gombosi TI, Sokolov IV, Velli M, Manchester W IV, DeZeeuw DL, Liewer P, Tth G, Luhmann J (2003) A Three-dimensional model of the solar wind incorporating solar magnetogram observations. Astrophys J 595(1):L57-L61. https://doi.org/10.1086/378878

Ruffenach A, Lavraud B, Farrugia CJ, Démoulin P, Dasso S, Owens MJ, Sauvaud J-A et al (2015) Statistical study of magnetic cloud erosion by magnetic reconnection. J Geophys Res 120:43-60. https://doi.org/10.1002/2014JA020628

Ruffenach A, Lavraud B, Owens MJ, Sauvaud J-A, Savani NP, Rouillard AP, Démoulin $P$ et al (2012) Multispacecraft observation of magnetic cloud erosion by magnetic reconnection during propagation: magnetic cloud erosion. J Geophys Res Space Phys 117(A9):n/a. https://doi.org/10.1029/2012 JA017624

Russell CT, Snare RC, Means JD, Pierce D, Dearborn D, Larson M, Barr G, Le G (1995) The GGS/POLAR magnetic fields investigation. Space Sci Rev 71(1-4): $563-582$

Ryan JM (2000) Long-duration solar gamma-ray flares. Space Sci Rev 93(3-4):581610

Ryan JM, Lee MA (1991) On the Transport and acceleration of solar flare particles in a coronal loop. Astrophys J 368:316-324

Sachdeva N, Subramanian P, Colaninno R, Vourlidas A (2015) CME propagation: where does aerodynamic drag 'take over'? Astrophys J 809:158. https://doi. org/10.1088/0004-637X/809/2/158

Sachdeva N, van der Holst B, Manchester WB, Tóth G, Chen Y, Lloveras DG, Vásquez AM et al (2019) Validation of the Alfvén wave solar atmosphere model (AWSOM) with observations from the low corona to $1 \mathrm{Au}$. Astrophys 887(1):83. https://doi.org/10.3847/1538-4357/ab4f5e

Salas-Matamoros C, Klein K-L, Rouillard AP (2016) Coronal mass ejection-related particle acceleration regions during a simple eruptive event. Astron Astrophys 590:A135

Salman TM, Lugaz N, Farrugia CJ, Winslow RM, Galvin AB, Schwadron NA (2018) Forecasting periods of strong southward magnetic field following interplanetary shocks. Space Weather 16:2004-2021. https://doi.org/10.1029/2 $0185 W 002056$

Sarkar R, Gopalswamy N, Srivastava N (2020) An observationally constrained analytical model for predicting the magnetic field vectors of interplanetary coronal mass ejections at 1 Au. Astrophys J 888(2):121. https://doi.org/10.384 7/1538-4357/ab5fd7

Savani NP, Owens MJ, Rouillard AP, Forsyth RJ, Davies JA (2010) Observational evidence of a coronal mass ejection distortion directly attributable to a structured solar wind. Astrophys J Lett 714:L128-L132. https://doi.org/10.1 088/2041-8205/714/1/L128

Savani NP, Owens MJ, Rouillard AP, Forsyth RJ, Kusano K, Shiota D, Kataoka R (2011) Evolution of coronal mass ejection morphology with increasing heliocentric distance. I. Geometrical analysis. Astrophys J 731:109. https://doi. org/10.1088/0004-637X/731/2/109

Savani NP, Vourlidas A, Richardson IG, Szabo A, Thompson BJ, Pulkkinen A, Mays ML, Nieves-Chinchilla T, Bothmer V (2017) Predicting the Magnetic vectors within coronal mass ejections arriving at Earth: 2. Geomagnetic response: $B$ \$_Itextrm Z \$ validation. Space Weather 15(2):441-461. https://doi.org/10.1 002/2016SW001458

Savani NP, Vourlidas A, Szabo A, Mays ML, Richardson IG, Thompson BJ, Pulkkinen A, Evans R, Nieves-Chinchilla T (2015) Predicting the magnetic vectors within coronal mass ejections arriving at Earth: 1. Initial architecture. Space Weather 13(6):374-385. https://doi.org/10.1002/2015SW001171

Schatten KH (1971) Current sheet magnetic model for the solar corona. Cosmic Electrodynamics 2:232-245 http://adsabs.harvard.edu/abs/1971CosEl...2..232S

Schatten KH, Wilcox JM, Ness NF (1969) A model of interplanetary and coronal magnetic fields. Sol Phys 6:442-455. https://doi.org/10.1007/BF00146478

Schmidt JM, Cargill PJ (2004) A numerical study of two interacting coronal mass ejections. Ann Geophys 22(6):2245-2254. https://doi.org/10.5194/a ngeo-22-2245-2004

Schmieder B, Aulanier G (2018) Solar active region electric currents before and during eruptive flares. 235:391-406. https://doi.org/10.1002/9781119324522. ch23

Schmieder B, Aulanier G, Vršnak B (2015) Flare-CME models: an observational perspective (invited review). Sol Phys 290(12):3457-3486. https://doi.org/10.1 007/s11207-015-0712-1

Schmieder B, Démoulin P, Aulanier G (2013) Solar filament eruptions and their physical role in triggering coronal mass ejections. Adv Space Res. 51(11): 1967-1980. https://doi.org/10.1016/j.asr.2012.12.026
Schmieder B, Kim RS, Grison B, Bocchialini K, Kwon RY, Poedts S, D'emoulin P (2020) Low Geo-Effectiveness of Fast Halo CMEs Related to the 12 X-Class Flares in 2002. J Geophys Res (Space Physics) 125(6):e27529. https://doi.org/1 0.1029/2019JA027529

Schrijver CJ (2007) A characteristic magnetic field pattern associated with all major solar flares and its use in flare forecasting. Astrophys J Lett 655:L117L120. https://doi.org/10.1086/511857

Schrijver CJ (2009) Driving major solar flares and eruptions: a review. Adv Space Res 43:739-755. https://doi.org/10.1016/j.asr.2008.11.004

Schwadron NA, Lee MA, Gorby M, Lugaz N, Spence HE, Desai M, Török T et al (2015) Particle acceleration at low coronal compression regions and shocks. Astrophys J 810(2):97

Schwadron NA, Rahmanifard F, Wilson J, Jordan AP, Spence HE, Joyce CJ, Blake JB et al (2018) Update on the worsening particle radiation environment observed by CRaTER and implications for future human deep-space exploration. Space Weather 16(3):289-303

Schwenn R (1983) Direct correlations between coronal transients and interplanetary disturbances. Space Sci Rev 34:85-99. https://doi.org/10.1007/ BF00221199

Schwenn R (2006) Space weather: the solar perspective. Living Rev Sol Phys 3:2. https://doi.org/10.12942//rsp-2006-2

Schwenn R, Lago AD, Huttunen E, Gonzalez WD (2005) The association of coronal mass ejections with their effects near the Earth. Astrophys J 23:1033-1059

Scolini C, Messerotti M, Poedts S, Rodriguez L (2018b) Halo coronal mass ejections during solar cycle 24: reconstruction of the global scenario and geoeffectiveness. J Space Weather Space Climate 8:A9. https://doi.org/10.1 051/swsc/2017046

Scolini C, Rodriguez L, Mierla M, Pomoell J, Poedts S (2019c) Observation-based modelling of magnetised coronal mass ejections with EUHFORIA. Astron Astrophys 626:A122. https://doi.org/10.1051/0004-6361/201935053

Scolini C, Verbeke C, Poedts S, Chané E, Pomoell J, Zuccarello FP (2018a) Effect of the initial shape of coronal mass ejections on 3-D MHD simulations and geoeffectiveness predictions. Space Weather 16(6):754-771. https://doi.org/1 0.1029/2018SW001806

Scolini, Camilla, Emmanuel Chané, Manuela Temmer, Emilia K. J. Kilpua, Karin Dissauer, Astrid M. Veronig, Erika Palmerio, et al. 2019a. "CME-CME interactions as sources of CME geo-effectiveness: the formation of the complex ejecta and intense geomagnetic storm in early September 2017." ArXiv:1911.10817 [Astro-Ph, Physics:Physics], November. http://arxiv.org/abs/1 911.10817.

Scolini, Camilla, Luciano Rodriguez, Manuela Temmer, Jingnan Guo, Mateja Dumbovic, Jens Pomoell, and Stefaan Poedts. 2019b. "Investigating the evolution and interactions of the September 2017 CME events with EUHFORIA," May, 1. http://adsabs.harvard.edu/abs/2019shin.confE...1S.

Seaton DB, Darnel JM (2018) Observations of an eruptive solar flare in the extended EUV solar corona. Astrophys J Lett 852:L9. https://doi.org/10.3847/2 041-8213/aaa28e

Selvakumaran R, Veenadhari B, Akiyama S, Megha P, Gopalswamy N, Yashiro S, Sandeep K, Mäkelä P, Xie H (2016) On the reduced geoeffectiveness of solar cycle 24: a moderate storm perspective. J Geophys Res (Space Physics) 121: 8188-8202. https://doi.org/10.1002/2016JA022885

Shanmugaraju A, Moon Y-J, Dryer M, Umapathy S (2003) On the kinematic evolution of flare-associated CMEs. Sol Phys 215(1):185-201. https://doi.org/1 0.1023/A:1024808819850

Shanmugaraju A, Prasanna Subramanian S, Vrsnak B, Syed Ibrahim M (2014) Interaction between two CMEs during $14-15$ February 2011 and their unusual radio signature. Sol Phys 289(12):4621-4632. https://doi.org/10.1007/ s11207-014-0591-x

Share GH, Murphy RJ, White SM, Tolbert AK, Dennis BR, Schwartz RA, Smart DF, Shea MA (2018) Characteristics of late-phase> $100 \mathrm{MeV}$ gamma-ray emission in solar eruptive events. Astrophys J 869(2):182

Sheeley NR Jr, Howard RA, Michels DJ, Koomen MJ, Schwenn R, Muehlhaeuser KH, Rosenbauer H (1985) Coronal mass ejections and interplanetary shocks. J Geophys Res 90:163-175. https://doi.org/10.1029/JA090iA01 p00163

Sheeley NR, Hakala WN, Wang Y-M (2000) Detection of coronal mass ejection associated shock waves in the outer corona. J Geophys Res 105:5081-5092. https://doi.org/10.1029/1999JA000338

Sheeley NR, Walters JH, Wang Y-M, Howard RA (1999) Continuous tracking of coronal outflows: two kinds of coronal mass ejections. J Geophys Res 104: 24739-24768. https://doi.org/10.1029/1999JA900308 
Shen C, Wang Y, Gui B, Ye P, Wang S (2011a) Kinematic evolution of a slow CME in corona viewed by STEREO-B on 8 October 2007. Sol Phys 269:389-400. https://doi.org/10.1007/s11207-011-9715-8

Shen C, Wang Y, Pan Z, Zhang M, Ye P, Wang S (2013a) Full Halo Coronal Mass ejections: do we need to correct the projection effect in terms of velocity? J Geophys Res (Space Physics) 118:6858-6865. https://doi.org/10.1002/2013JA 018872

Shen C, Wang Y, Wang S, Liu Y, Liu R, Vourlidas A, Miao B, Ye P, Liu J, Zhou Z (2012a) Super-Elastic collision of large-scale magnetized plasmoids in the heliosphere. Nat Phys 8(12):923-928. https://doi.org/10.1038/nphys2440

Shen C, Xu M, Wang Y, Chi Y, Luo B (2018a) Why the Shock-ICME complex structure is important: learning from the early 2017 September CMES. Astrophys J 861 (1):28. https://doi.org/10.3847/1538-4357/aac204

Shen F, Chenglong S, Yuming W, Xueshang F, Changqing X (2013b) Could the collision of CMEs in the heliosphere be super-elastic? Validation through three-dimensional simulations: super-elastic collision of CMEs. Geophys Res Lett 40(8):1457-1461. https://doi.org/10.1002/grl.50336

Shen F, Feng X, Wu ST, Xiang C (2007) Three-dimensional MHD simulation of CMEs in three-dimensional background solar wind with the self-consistent structure on the source surface as input: numerical simulation of the January 1997 Sun-Earth connection event: numerical study of January 1997 CME. J Geophys Res Space Phys 112(A6):n/a. https://doi.org/10.1029/2006JA012164

Shen F, Feng XS, Wang Y, Wu ST, Song WB, Guo JP, Zhou YF (2011C) Threedimensional MHD simulation of two coronal mass ejections' propagation and interaction using a successive magnetized plasma blobs model: a 3-D simulation of interaction between two CMEs. J Geophys Res Space Phys 116(A9):n/a. https://doi.org/10.1029/2011JA016584

Shen F, Feng XS, Wu ST, Xiang CQ, Song WB (2011b) Three-Dimensional MHD Simulation of the Evolution of the April 2000 CME Event and Its Induced Shocks Using a Magnetized Plasma Blob Model: A 3-D MHD SIMULATION OF APRIL 2000 CME EVENT. J Geophys Res Space Phys 116(A4):n/a. https://doi. org/10.1029/2010JA015809

Shen F, Shen C, Wang Y, Feng X, Xiang C (2013c) Could the collision of CMEs in the heliosphere be super-elastic? Validation through three-dimensional simulations: super-elastic collision of CMEs. Geophys Res Lett 40(8):1457-1461. https://doi.org/10.1002/grl.50336

Shen F, Shen C, Zhang J, Hess P, Wang Y, Feng X, Cheng H, Yang Y (2014) Evolution of the 12 July 2012 CME from the Sun to the Earth: dataconstrained three-dimensional mhd simulations: 3D simulation to 2012 July 12 CME event. J Geophys Res Space Phys 119(9):7128-7141. https://doi.org/1 0.1002/2014JA020365

Shen F, Wang Y, Shen C, Feng X (2016) Turn on the super-elastic collision nature of coronal mass ejections through low approaching speed. Sci Rep 6(1): 19576. https://doi.org/10.1038/srep19576

Shen F, Wang Y, Shen C, Feng X (2017) On the collision nature of two coronal mass ejections: a review. Sol Phys 292(8):104. https://doi.org/10.1007/s11207017-1129-9

Shen F, Wu ST, Feng X, Wu C-C (2012) Acceleration and deceleration of coronal mass ejections during propagation and interaction: acceleration and deceleration of CME. J Geophys Res Space Phys 117(A11):n/a. https://doi. org/10.1029/2012JA017776

Shen F, Yang Z, Zhang J, Wei W, Feng X (2018b) Three-dimensional MHD simulation of solar wind using a new boundary treatment: comparison with in situ data at Earth. Astrophys J 866(1):18. https://doi.org/10.3847/1538-43 57/aad806

Shibata K, Magara T (2011) Solar flares: magnetohydrodynamic processes. Living Rev Sol Phys 8:6. https://doi.org/10.12942/lrsp-2011-6

Shiokawa K, Katya G (2020) A review of the SCOSTEP's 5-year scientific program VarSITI-Variability of the Sun and Its Terrestrial Impact. Prog Earth Planet Sci 8(1):21. https://doi.org/10.1186/s40645-021-00410-1

Shiota D, Kataoka R (2016) magnetohydrodynamic simulation of interplanetary propagation of multiple coronal mass ejections with internal magnetic flux rope (SUSANOO-CME): development of SUSANOO-CME MODEL. Space Weather 14(2):56-75. https://doi.org/10.1002/2015SW001308

Shiota D, Kataoka R, Miyoshi Y, Hara T, Tao C, Masunaga K, Futaana Y, Terada N (2014) Inner heliosphere MHD modeling system applicable to space weather forecasting for the other planets. Space Weather 12(4):187-204. https://doi. org/10.1002/2013SW000989

Sindhuja G, Gopalswamy N (2020) A study of the observational properties of coronal mass ejection flux ropes near the Sun. Astrophys J 889:104. https:// doi.org/10.3847/1538-4357/ab620f
Singh T, Yalim MS, Pogorelov NV, Gopalswamy N (2020) A modified spheromak model suitable for coronal mass ejection simulations. Astrophys J 894:49. https://doi.org/10.3847/1538-4357/ab845f

Singh T, Yalim MS, Pogorelov NV, Gopalswamy N (2019) Simulating solar coronal mass ejections constrained by observations of their speed and poloidal flux. Astrophys J Lett 875:L17. https://doi.org/10.3847/2041-8213/ab14e9

Siscoe G, Schwenn R (2006) CME disturbance forecasting. Space Sci Rev 123:453470. https://doi.org/10.1007/s11214-006-9024-y

Smith CW, L'Heureux J, Ness NF, Acuña MH, Burlaga LF, Scheifele J (1998) The ACE magnetic fields experiment. Space Sci Rev 86:613-632. https://doi.org/1 0.1023/A:1005092216668

Sokolov IV, van der Holst B, Oran R, Downs C, Roussev II, Jin M, Manchester WB, Evans RM, Gombosi TI (2013) Magnetohydrodynamic waves and coronal heating: unifying empirical and mhd turbulence models. Astrophys J 764(1): 23. https://doi.org/10.1088/0004-637X/764/1/23

Song HQ, Cheng X, Chen Y, Zhang J, Wang B, Li LP, Li B, Hu Q, Li G (2017) The three-part structure of a filament-unrelated solar coronal mass ejection Astrophys J 848(1):21. https://doi.org/10.3847/1538-4357/aa8d1a

Song HQ, Zhang J, Cheng X, Li LP, Tang YZ, Wang B, Zheng RS, Chen Y (2019) On the nature of the bright core of solar coronal mass ejections. Astrophys J 883:43. https://doi.org/10.3847/1538-4357/ab304c

Song HQ, Zhou ZJ, Li LP, Cheng X, Zhang J, Chen Y, Chen CX, Ma XW, Wang B, Zheng RS (2018) The reversal of a solar prominence rotation about its ascending direction during a failed eruption. Astrophys J Lett 864:L37. https://doi.org/10.3847/2041-8213/aade49

Srivastava N, Venkatakrishnan P (2004) Solar and interplanetary sources of major geomagnetic storms during 1996-2002. J Geophys Res (Space Physics) 109: A10103. https://doi.org/10.1029/2003JA010175

Stone EC, Frandsen AM, Mewaldt RA, Christian ER, Margolies D, Ormes JF, Snow F (1998) The advanced composition explorer. Space Sci Rev 86(1-4):1-22

Strauss RDT, Dresing N, Engelbrecht NE (2017) Perpendicular diffusion of solar energetic particles: model results and implications for electrons. Astrophys J 837(1):43

Subramanian P, Arunbabu KP, Vourlidas A, Mauriya A (2014) Self-similar expansion of solar coronal mass ejections: implications for Lorentz self-force driving. Astrophys J 790:125. https://doi.org/10.1088/0004-637X/790/2/125

Subramanian S, Madjarska MS, Doyle JG (2010) Coronal hole boundaries evolution at small scales. II. XRT View. Can small-scale outflows at CHBs be a source of the slow solar wind. Astron Astrophys 516:A50. https://doi.org/10.1 051/0004-6361/200913624

Sudar D, Vršnak B, Dumbović M (2016) Predicting coronal mass ejections transit times to earth with neural network. Mon Not R Astron Soc 456:1542-1548. https://doi.org/10.1093/mnras/stv2782

Sun X, Bobra MG, Todd Hoeksema J, Yang L, Li Y, Shen C, Couvidat S, Norton AA, Fisher GH (2015) Why is the great solar active region 12192 flare-rich but CME-poor? Astrophys J Lett 804:L28. https://doi.org/10.1088/2041-8205/ 804/2/L28

Susino R, Bemporad A (2016) Determination of coronal mass ejection physical parameters from a combination of polarized visible light and UV Ly a observations. Astrophys J 830(2):58. https://doi.org/10.3847/0004-637X/830/2/58

Susino R, Bemporad A, Dolei S, Vourlidas A (2013) Study of a coronal mass ejection with SOHO/UVCS and STEREO data. Adv Space Res 52(5):957-962. https://doi.org/10.1016/j.asr.2013.05.017

Suzuki J, Welsch BT, Li Y (2012) Are decaying magnetic fields above active regions related to coronal mass ejection onset? Astrophys J 758:22. https:// doi.org/10.1088/0004-637X/758/1/22

Syntelis P, Archontis V, Tsinganos K (2017) Recurrent CME-like eruptions in emerging flux regions. I. On the mechanism of eruptions. Astrophys J 850:95. https://doi.org/10.3847/1538-4357/aa9612

Syntelis P, Gontikakis C, Patsourakos S, Tsinganos K (2016) The spectroscopic imprint of the pre-eruptive configuration resulting into two major coronal mass ejections. Astron Astrophys 588:A16. https://doi.org/10.1051/0004-63 $61 / 201526829$

Taktakishvili A, Pulkkinen A, MacNeice P, Kuznetsova M, Hesse M, Odstrcil D (2011) Modeling of coronal mass ejections that caused particularly large geomagnetic storms using ENLIL heliosphere cone model: modeling CMEs caused strong geomagnetic storms. Space Weather 9(6):n/a. https://doi.org/1 0.1029/2010SW000642

Tan LC, Malandraki OE, Reames DV, Ng CK, Wang L, Dorrian G (2012) Use of incident and reflected solar particle beams to trace the topology of magnetic clouds. Astrophys J 750(2):146 
Tan LC, Malandraki OE, Shao X (2017) Joint Ne/O and Fe/O analysis to diagnose large solar energetic particle events during solar cycle 23. Astrophys J 835(2): 192

Tan LC, Mason GM, Klecker B, Hovestadt D (1989) Seed population for about 1 $\mathrm{MeV}$ per nucleon heavy ions accelerated by interplanetary shocks. Astrophys J 345:572-583

Temmer M (2016) Kinematical properties of coronal mass ejections. Astron Nachr. 337:1010. https://doi.org/10.1002/asna.201612425

Temmer M, Hinterreiter J, Reiss MA (2018) Coronal hole evolution from multiviewpoint data as input for a STEREO solar wind speed persistence model. J Space Weather Space Climate 8:A18. https://doi.org/10.1051/swsc/2018007

Temmer M, Holzknecht L, Dumbović M, Vršnak B, Sachdeva N, Heinemann SG, Dissauer $\mathrm{K}$ et al (2021) Deriving CME density from remote sensing data and comparison to in-situ measurements. J Geophys Res Space Phys 126(1): e2020JA028380. https://doi.org/10.1029/2020JA028380

Temmer M, Reiss MA, Nikolic L, Hofmeister SJ, Veronig AM (2017) Preconditioning of interplanetary space due to transient CME disturbances. Apj 835(2):141. https://doi.org/10.3847/1538-4357/835/2/141

Temmer M, Thalmann JK, Dissauer K, Veronig AM, Tschernitz J, Hinterreiter J, Rodriguez L (2017a) On Flare-CME characteristics from Sun to Earth combining remote-sensing image data with in situ measurements supported by modeling. Sol Phys 292(7):93. https://doi.org/10.1007/s11207-017-1112-5

Temmer M, Veronig AM, Kontar EP, Krucker S, Vršnak B (2010) Combined STEREO/ RHESSI Study of Coronal Mass Ejection Acceleration and Particle Acceleration in Solar Flares. apj 712(2):1410-1420. https://doi.org/10.1088/0004-637X/ $712 / 2 / 1410$

Temmer M, Veronig AM, Peinhart V, Vršnak B (2014) Asymmetry in the CME-CME interaction process for the events from 2011 February 14-15. Astrophys J 785(2):85. https://doi.org/10.1088/0004-637X/785/2/85

Temmer M, Veronig AM, Vršnak B, Rybák J, Gömöry P, Stoiser S, Maričić D (2008) Acceleration in fast halo CMEs and synchronized flare HXR bursts. Astrophys J Lett 673:L95. https://doi.org/10.1086/527414

Temmer M, Vršnak B, Rollett T, Bein B, de Koning CA, Liu Y, Bosman E et al (2012) Characteristics of kinematics of a coronal mass ejection during the 2010 August 1 CME-CME interaction event. Astrophys J 749:57. https://doi.org/10.1 088/0004-637X/749/1/57

Temmer M, Vršnak B, Veronig AM (2007) Periodic appearance of coronal holes and the related variation of solar wind parameters. Sol Phys 241:371-383. https://doi.org/10.1007/s11207-007-0336-1

Thalmann JK, Moraitis K, Linan L, Pariat E, Valori G, Dalmasse K (2019) Magnetic helicity budget of solar active regions prolific of eruptive and confined flares. Astrophys J 887:64. https://doi.org/10.3847/1538-4357/ab4e15

Thernisien A (2011) Implementation of the graduated cylindrical shell model for the three-dimensional reconstruction of coronal mass ejections. Astrophys Suppl 194:33. https://doi.org/10.1088/0067-0049/194/2/33

Thernisien A, Vourlidas A, Howard RA (2009) Forward modeling of coronal mass ejections using STEREO/SECCHI data. Sol Phys 256(1-2):111-130. https://doi. org/10.1007/s11207-009-9346-5

Thernisien AFR, Howard RA, Vourlidas A (2006) Modeling of flux rope coronal mass ejections. Astrophys J 652(1):763

Thompson BJ, Cliver EW, Nitta N, Delannée C, Delaboudinière J-P (2000) Coronal dimmings and energetic CMEs in April-May 1998. Geophys Res Lett 27:14311434. https://doi.org/10.1029/1999GL003668

Thompson BJ, Gurman JB, Neupert WM, Newmark JS, Delaboudinière J-P, OCS C, Stezelberger S, Dere KP, Howard RA, Michels DJ (1999) [ITAL]SOHO[/ITAL]/EIT Observations of the 1997 April 7 coronal transient: possible evidence of coronal moreton waves. Astrophys J 517(2):L151-L154. https://doi.org/10.1 086/312030

Thompson BJ, Myers DC (2009) A catalog of coronal 'eit wave' transients. Astrophys J Suppl Ser 183(2):225-243. https://doi.org/10.1088/0067-0049/1 $83 / 2 / 225$

Titov VS, Demoulin P (1999) Basic topology of twisted magnetic configurations in solar flares. 351:707-720

Titov VS, Török T, Mikic Z, Linker JA (2014) A method for embedding circular force-free flux ropes in potential magnetic fields. Astrophys J 790(2):163. https://doi.org/10.1088/0004-637X/790/2/163

Tokumaru M, Satonaka D, K'i F, Hayashi K, Hakamada K (2017) Relation between coronal hole areas and solar wind speeds derived from interplanetary scintillation measurements. Sol Phys 292:41. https://doi.org/10.1007/s11207017-1066-7
Török T, Berger MA, Kliem B (2010) The writhe of helical structures in the solar corona. Astron Astrophys 516:A49. https://doi.org/10.1051/0004-6361/200913 578

Török T, Downs C, Linker JA, Lionello R, Titov VS, Mikić Z, Riley P, Caplan RM, Wijaya J (2018) Sun-to-Earth MHD simulation of the 2000 July 14 'Bastille Day' eruption. Astrophys J 856(1):75. https://doi.org/10.3847/1538-4357/aab3 $6 \mathrm{~d}$

Török T, Kliem B (2003) The evolution of twisting coronal magnetic flux tubes. Astron Astrophys 406(3):1043-1059. https://doi.org/10.1051/0004-6361:2003 0692

Török T, Kliem B (2005) Confined and ejective eruptions of kink-unstable flux ropes. Astrophys J 630(1):L97-L100. https://doi.org/10.1086/462412

Török T, Kliem B, Titov VS (2004) Ideal kink instability of a magnetic loop equilibrium. Astron Astrophys 413(3):L27-L30. https://doi.org/10.1051/0004-63 61:20031691

Török T, Temmer M, Valori G, Veronig AM, van Driel-Gesztelyi L, Vršnak B (2013) Initiation of coronal mass ejections by sunspot rotation. Sol Phys 286(2):453477. https://doi.org/10.1007/s11207-013-0269-9

Tóth G, van der Holst B, Sokolov IV, De Zeeuw DL, Gombosi TI, Fang F, Ward B. Manchester, et al. (2012) Adaptive numerical algorithms in space weather modeling. J Comput Phys 231(3):870-903. https://doi.org/10.1016/j.jcp.2011. 02.006

Tóth GB, de Zeeuw DL, Gombosi TI, Manchester WB, Ridley AJ, Sokolov IV, Roussev II (2007) Sun-to-thermosphere simulation of the 28-30 October 2003 storm with the space weather modeling framework. Space Weather 5:06003. https://doi.org/10.1029/2006SW000272

Tousey R (1973) The solar corona. In: Rycroft MJ, Runcorn SK (eds) Space Research XIII, Proceedings of Open Meetings of Working Groups on Physical Sciences of the 15th Plenary Meeting of COSPAR, Madrid, Spain, 1024 May, 1972, vol 2. Akademie-Verlag, Berlin, pp 713-730 http://adsabs.harvard.edu/a bs/1973spre.conf..713T

Tripathi D, Bothmer V, Cremades H (2004) The basic characteristics of EUV posteruptive arcades and their role as tracers of coronal mass ejection source regions. Astron Astrophy 422(1):337-349. https://doi.org/10.1051/0004-6361:2 0035815

Trottet G, Samwel S, Klein K-L, Dudok De Wit T, Miteva R (2015) Statistical evidence for contributions of flares and coronal mass ejections to major solar energetic particle events. Sol Phys 290(3):819-839

Tschernitz J, Veronig AM, Thalmann JK, Hinterreiter J, Pötzi W (2018) Reconnection fluxes in eruptive and confined flares and implications for superflares on the Sun. Astrophys J 853(1):41. https://doi.org/10.3847/1538-43 57/aaa199

Tsurutani BT, Gonzalez WD, Gonzalez ALC, Guarnieri FL, Gopalswamy N, Grande M, Kamide $Y$ et al (2006) Corotating solar wind streams and recurrent geomagnetic activity: a review. J Geophys Res (Space Physics) 111:A07S01. https://doi.org/10.1029/2005JA011273

Tsurutani BT, Gonzalez WD, Tang F, Akasofu SI, Smith EJ (1988) Origin of interplanetary southward magnetic fields responsible for major magnetic storms near solar maximum (1978-1979). J Geophys Res 93:8519-8531. https://doi.org/10.1029/JA093iA08p08519

Tsurutani BT, Lakhina GS, Sen A, Hellinger P, Glassmeier K-H, Mannucci AJ (2018) A review of Alfvénic turbulence in high-speed solar wind streams: hints from cometary plasma turbulence. J Geophys Res (Space Physics) 123:2458-2492. https://doi.org/10.1002/2017JA024203

Tsurutani BT, Lin RP (1985) Acceleration of $>47 \mathrm{KeV}$ ions and $>2 \mathrm{KeV}$ electron by interplanetary shocks at 1 AU. J Geophys Res Space Phys 90(A1):1-11

Tu C-Y, Cheng Z, Marsch E, Xia L-D, Liang Z, Wang J-X, Wilhelm K (2005) Solar wind origin in coronal funnels. Science 308(5721):519-523. https://doi.org/1 $0.1126 /$ science. 1109447

Tun SD, Vourlidas A (2013) Derivation of the magnetic field in a coronal mass ejection core via multi-frequency radio imaging. Astrophys J 766(2):130 https://doi.org/10.1088/0004-637X/766/2/130

Tylka AJ, Cohen CMS, Dietrich WF, Lee MA, Maclennan CG, Mewaldt RA, Ng CK, Reames DV (2005) Shock geometry, seed populations, and the origin of variable elemental composition at high energies in large gradual solar particle events. Astrophys J 625(1):474

Tylka AJ, Dietrich W, Atwell W (2010) Band Function representations of solar proton spectra in ground-level events. Cosp 38:4

Tylka AJ, Lee MA (2006) A model for spectral and compositional variability at high energies in large, gradual solar particle events. Astrophys J 646(2):1319 
Tylka AJ, Malandraki OE, Dorrian G, Ko Y-K, Marsden RG, Ng CK, Tranquille C (2013) Initial Fe/O enhancements in large, gradual, solar energetic particle events: observations from wind and Ulysses. Sol Phys 285(1-2):251-267

Tylka AJ, Boberg PR, McGuire RE, Ng CK, Reames DV (2000) Temporal evolution in the spectra of gradual solar energetic particle events. In: AIP Conference Proceedings, vol 528. American Institute of Physics, pp 147-152

Tylka AJ, Reames DV, Ng CK (1999) Observations of systematic temporal evolution in elemental composition during gradual solar energetic particle events. Geophys Res Lett 26(14):2141-2144

Tziotziou K, Georgoulis MK, Raouafi N-E (2012) The magnetic energy-helicity diagram of solar active regions. Astrophys J Lett 759:L4. https://doi.org/10.1 088/2041-8205/759/1/L4

Ugarte-Urra I, Warren HP, Winebarger AR (2007) The magnetic topology of coronal mass ejection sources. Astrophys J 662(2):1293-1301 https://doi. org/10.1086/514814

Usmanov AV, Matthaeus WH, Goldstein ML, Chhiber R (2018) The steady global corona and solar wind: a three-dimensional MHD simulation with turbulence transport and heating. Astrophys J 865(1):25 https://doi.org/10.3847/1538-43 57/aad687

van der Holst B, Manchester WB, Frazin RA, Vásquez AM, Tóth G, Gombosi TI (2010) A data-driven, two-temperature solar wind model with Alfvén waves. Astrophys J 725(1):1373-1383 https://doi.org/10.1088/0004-637X/725/1/1373

van der Holst B, Sokolov IV, Meng X, Jin M, Manchester WB IV, Tóth G, Gombosi TI (2014) Alfvén wave solar model (AWSoM): coronal heating. Astrophys J 782(2):81 https://doi.org/10.1088/0004-637X/782/2/81

Vandas M, Romashets E (2015) Comparative study of a constant-alpha force-free field and its approximations in an ideal toroid. Astron Astrophys 580:A123 https://doi.org/10.1051/0004-6361/201526242

Vandas M, Romashets E (2017a) Toroidal Flux ropes with elliptical cross sections and their magnetic helicity. Sol Phys 292(9):129 https://doi.org/10.1007/s112 07-017-1149-5

Vandas M, Romashets E (2017b) Magnetic cloud fit by uniform-twist toroidal flux ropes. Astron Astrophys 608:A118 https://doi.org/10.1051/0004-6361/201 731412

Vandas M, Romashets E, Geranios A (2010) How do fits of simulated magnetic clouds correspond to their real shapes in 3-D? Ann Geophys 28(8):1581-1588 https://doi.org/10.5194/angeo-28-1581-2010

Vandas M, Romashets E, Geranios A (2015) Modeling of magnetic cloud expansion. Astron Astrophys 583:A78 https://doi.org/10.1051/0004-6361/2 01425594

Vandas M, Romashets EP (2019) Interplanetary flux ropes of any twist distribution. Astron Astrophys 627:A90 https://doi.org/10.1051/0004-6361/201935216

Vandas M, Romashets EP, Watari S, Geranios A, Antoniadou E, Zacharopoulou O (2006) Comparison of force-free flux rope models with observations of magnetic clouds. Adv Space Res 38(3):441-446 https://doi.org/10.1016/j.asr.2 004.11 .026

Vanninathan K, Veronig AM, Dissauer K, Temmer M (2018) Plasma diagnostics of coronal dimming events. Astrophys J 857(1):62 https://doi.org/10.3847/153 8-4357/aab09a

Vasantharaju N, Vemareddy P, Ravindra B, Doddamani VH (2019) Finding the critical decay index in solar prominence eruptions. Astrophys J 885:89 https://doi.org/10.3847/1538-4357/ab4793

Verbeeck C, Delouille V, Mampaey B, De Visscher R (2014) The SPoCA-suite: software for extraction, characterization, and tracking of active regions and coronal holes on EUV images. Aap 561:A29 https://doi.org/10.1051/0004-63 $61 / 201321243$

Verbeke C, Mays ML, Temmer M, Bingham S, Steenburgh R, Dumbović M, Núñez $M$ et al (2019) Benchmarking CME arrival time and impact: progress on metadata, metrics, and events. Space Weather 17:6-26 https://doi.org/10.102 9/2018SW002046

Verbeke C, Pomoell J, Poedts S (2019a) The evolution of coronal mass ejections in the inner heliosphere: implementing the spheromak model with EUHFORIA. Astron Astrophys 627:A111 https://doi.org/10.1051/0004-6361/201 834702

Veronig AM, Podladchikova T, Dissauer K, Temmer M, Seaton DB, Long D, Guo J, Vršnak B, Harra L, Kliem B (2018) Genesis and impulsive evolution of the 2017 September 10 coronal mass ejection. Astrophys J 868:107 https://doi.org/10.3 847/1538-4357/aaeac5

Von Steiger R, Schwadron NA, Fisk LA, Geiss J, Gloeckler G, Hefti S, Wilken B, Wimmer-Schweingruber RR, Zurbuchen TH (2000) Composition of quasi- stationary solar wind flows from Ulysses/solar wind ion composition spectrometer. J Geophys Res Space Phys 105(A12):27217-27238

Vourlidas A, Balmaceda LA, Stenborg G, Lago AD (2017) Multi-viewpoint coronal mass ejection catalog based on STEREO COR2 observations. Astrophys J 838(2):141

Vourlidas A, Colaninno R, Nieves-Chinchilla T, Stenborg G (2011) The first observation of a rapidly rotating coronal mass ejection in the middle corona. Astrophys J Lett. 733:L23 https://doi.org/10.1088/2041-8205/733/2/L23

Vourlidas A, Howard RA, Esfandiari E, Patsourakos S, Yashiro S, Michalek G (2010) Comprehensive analysis of coronal mass ejection mass and energy properties over a full solar cycle. Astrophys J 722:1522-1538 https://doi.org/1 0.1088/0004-637X/722/2/1522

Vourlidas A, Lynch BJ, Howard RA, Li Y (2013) How many CMEs have flux ropes? deciphering the signatures of shocks, flux ropes, and prominences in coronagraph observations of CMEs. Sol Phys 284:179-201 https://doi.org/10.1 007/s11207-012-0084-8

Vourlidas A, Patsourakos S, Savani NP (2019) Predicting the geoeffective properties of coronal mass ejections: current status, open issues and path forward. Philos Trans R Soc A Math Phys Eng Sci 377(2148):20180096 https:// doi.org/10.1098/rsta.2018.0096

Vourlidas A, Subramanian P, Dere KP, Howard RA (2000) Large-angle spectrometric coronagraph measurements of the energetics of coronal mass ejections. Astrophys J 534:456-467 https://doi.org/10.1086/308747

Vršnak B (1990) Eruptive instability of cylindrical prominences. Sol Phys 129(2): 295-312 https://doi.org/10.1007/BF00159042

Vršnak B (2008) Processes and mechanisms governing the initiation and propagation of CMEs. Ann Geophysicae 26(10):3089-3101 https://doi.org/10. 5194/angeo-26-3089-2008

Vršnak B (2016) Solar eruptions: the CME-flare relationship. Astronomische Nachr 337(10):1002-1009 https://doi.org/10.1002/asna.201612424

Vršnak B (2019) Gradual pre-eruptive phase of solar coronal eruptions. Front Astron Space Sci 6:28 https://doi.org/10.3389/fspas.2019.00028

Vršnak B, Dumbović M, Čalogović J, Verbanac G, Beljan IP (2017) Geomagnetic effects of corotating interaction regions. Sol Phys 292:140 https://doi.org/10.1 007/s11207-017-1165-5

Vršnak B, Maričić D, Stanger AL, Veronig A (2004) Coronal mass ejection of 15 May 2001: II. Coupling of the Cme acceleration and the flare energy release. Sol Phys 225(2):355-378 https://doi.org/10.1007/s11207-004-4995-x

Vršnak B, Sudar D, Ruždjak D (2005) The CME-flare relationship: are there really two types of CMEs? Astron Astrophys 435:1149-1157 https://doi.org/10.1051/ 0004-6361:20042166

Vršnak B, Temmer M, Veronig AM (2007a) Coronal holes and solar wind highspeed streams: I. Forecasting the solar wind parameters. Sol Phys 240(2):315$330 \mathrm{https}: / /$ doi.org/10.1007/s11207-007-0285-8

Vršnak B, Temmer M, Veronig AM (2007b) Coronal Holes and solar wind highspeed streams: II. Forecasting the geomagnetic effects. Sol Phys 240:331-346 https://doi.org/10.1007/s11207-007-0311-x

Vršnak B, Temmer M, Žic T, Taktakishvili A, Dumbović M, Möstl C, Veronig AM, Mays ML, Odstrčil D (2014) Heliospheric Propagation of coronal mass ejections: comparison of numerical WSA-ENLIL+Cone model and analytical drag-based model. Astrophys J Suppl 213:21 https://doi.org/10.1088/0067$0049 / 213 / 2 / 21$

Vršnak B, Žic T (2007) Transit times of interplanetary coronal mass ejections and the solar wind speed. Astron Astrophys 472:937-943 https://doi.org/10.1051/ 0004-6361:20077499

Vršnak B, Žic T, Lulić S, Temmer M, Veronig AM (2016) Formation of coronal large-amplitude waves and the chromospheric response. Sol Phys 291:89115 https://doi.org/10.1007/s11207-015-0822-9

Vršnak B, Žic T, Vrbanec D, Temmer M, Rollett T, Möstl C, Veronig A et al (2013) Propagation of interplanetary coronal mass ejections: the drag-based model. Sol Phys 285:295-315 https://doi.org/10.1007/s11207-012-0035-4

Wang C, Rosen IG, Tsurutani BT, Verkhoglyadova OP, Meng X, Mannucci AJ (2016a) Statistical characterization of ionosphere anomalies and their relationship to space weather events. J Space Weather Space Climate 6:A5 https://doi.org/10.1051/swsc/2015046

Wang D, Liu R, Wang Y, Liu K, Chen J, Liu J, Zhou Z, Zhang M (2017) Critical height of the torus instability in two-ribbon solar flares. Astrophys J Lett 843 : L9 https://doi.org/10.3847/2041-8213/aa79f0

Wang Y, Qin G (2015) Estimation of the release time of solar energetic particles near the Sun. Astrophys J 799(1):111 
Wang Y, Shen C, Liu R, Liu J, Guo J, Li X, Xu M, Hu Q, Zhang T (2018) Understanding the twist distribution inside magnetic flux ropes by anatomizing an interplanetary magnetic cloud: twist distribution in an interplanetary MC. J Geophys Res Space Phys 123(5):3238-3261 https://doi. org/10.1002/2017JA024971

Wang Y, Shen C, Wang S, Ye P (2004) Deflection of coronal mass ejection in the interplanetary medium. Sol Phys 222:329-343 https://doi.org/10.1023/B:SOLA. 0000043576.21942.aa

Wang Y, Wang B, Shen C, Shen F, Lugaz N (2014) Deflected Propagation of a coronal mass ejection from the corona to interplanetary space. J Geophys Res (Space Physics) 119:5117-5132 https://doi.org/10.1002/2013JA019537

Wang Y, Xue X, Shen C, Ye P, Wang S, Zhang J (2006) Impact of major coronal mass ejections on geospace during 2005 September 7-13. Astrophys J 646: 625-633 https://doi.org/10.1086/504676

Wang Y, Zhang J (2007) A comparative study between eruptive X-class flares associated with coronal mass ejections and confined X-class flares. Astrophys J 665:1428-1438 https://doi.org/10.1086/519765

Wang Y, Zhang J, Shen C (2009) An Analytical model probing the internal state of coronal mass ejections based on observations of their expansions and propagations: CME internal state. J Geophys Res Space Phys 114(A10):n/a https://doi.org/10.1029/2009JA014360

Wang Y, Zhang Q, Liu J, Shen C, Shen F, Yang Z, Zic T et al (2016b) On the propagation of a geoeffective coronal mass ejection during 15-17 March 2015. J Geophys Res (Space Physics) 121:7423-7434 https://doi.org/10.1002/2 016JA022924

Wang Y, Zheng H, Wang S, Ye P (2005) MHD Simulation of the formation and propagation of multiple magnetic clouds in the heliosphere. Astron Astrophys 434(1):309-316 https://doi.org/10.1051/0004-6361:20041423

Wang Y, Zhou Z, Shen C, Liu R, Wang S (2015) Investigating Plasma motion of magnetic clouds at $1 \mathrm{AU}$ through a velocity-modified cylindrical force-free flux rope model. J Geophys Res (Space Physics) 120(3):1543-1565 https://doi. org/10.1002/2014JA020494

Wang Y, Zhuang B, Hu Q, Liu R, Shen C, Chi Y (2016c) On the twists of interplanetary magnetic flux ropes observed at $1 \mathrm{AU}$. J Geophys Res (Space Physics) 121(10):9316-9339 https://doi.org/10.1002/2016JA023075

Wang Y, Zhuang B, Hu Q, Liu R, Shen C, Chi Y (2016d) On the twists of interplanetary magnetic flux ropes observed at $1 \mathrm{AU}$ : twists of interplanetary MFRs. J Geophys Res Space Phys 121(10):9316-9339 https://doi.org/10.1002/2 016JA023075

Wang YM (2003) Multiple magnetic clouds: several examples during March-April 2001. J Geophys Res 108(A10):1370 https://doi.org/10.1029/2003JA009850

Wang Y-M, Colaninno R (2014) Is solar cycle 24 producing more coronal mass ejections than cycle 23? Astrophys J Lett 784:L27 https://doi.org/10.1088/2 041-8205/784/2/L27

Wang Y-M, Sheeley NR Jr (1990) Solar wind speed and coronal flux-tube expansion. Apj 355:726 https://doi.org/10.1086/168805

Wang YM, Wang S, Ye PZ (2002) Multiple magnetic clouds in interplanetary space. Sol Phys 211(1/2):333-344 https://doi.org/10.1023/A:1022404425398

Warmuth A (2015) Large-scale globally propagating coronal waves. Living Rev Sol Phys 12(1):3 https://doi.org/10.1007//rsp-2015-3

Watari S (2018) Intense geomagnetic storms associated with coronal holes under the weak solar-wind conditions of cycle 24. Sol Phys 293:23 https://doi.org/1 0.1007/s11207-018-1248-y

Webb D, Nitta N (2017) Understanding problem forecasts of ISEST campaign flare-CME events. Sol Phys 292:142 https://doi.org/10.1007/s11207-017-1166-4

Webb DF, Howard TA (2012) Coronal mass ejections: observations. Living Rev Sol Phys 9(1):3 https://doi.org/10.12942//rsp-2012-3

Webb DF, Hundhausen AJ (1987) Activity associated with the solar origin of coronal mass ejections. Sol Phys 108(2):383-401 https://doi.org/10.1007/ BF00214170

Webb DF, Kahler SW, McIntosh PS, Klimchuck JA (1997) Large-scale structures and multiple neutral lines associated with coronal mass ejections. J Geophys Res 102:24161-24174 https://doi.org/10.1029/97JA01867

Webb DF, Lepping RP, Burlaga LF, DeForest CE, Larson DE, Martin SF, Plunkett SP, Rust DM (2000) The origin and development of the May 1997 magnetic cloud. J Geophys Res Space Phys 105(A12):27251-27259 https://doi.org/10.1 029/2000JA000021

Webb DF, Möstl C, Jackson BV, Bisi MM, Howard TA, Mulligan T, Jensen EA et al (2013) Heliospheric imaging of 3D density structures during the multiple coronal mass ejections of late July to early August 2010. Sol Phys 285(1-2): 317-348 https://doi.org/10.1007/s11207-013-0260-5
Welsch BT (2018) Flux Accretion and Coronal Mass Ejection Dynamics. 293(7):113 https://doi.org/10.1007/s11207-018-1329-y

Werner ALE, Yordanova E, Dimmock AP, Temmer M (2019) Modeling the multiple CME interaction event on 6-9 September 2017 with WSA-ENLIL+Cone. Space Weather 17(2):357-369 https://doi.org/10.1029/2018SW001993

Wiegelmann T, Solanki SK (2004) Similarities and differences between coronal holes and the quiet sun: are loop statistics the key? Sol Phys 225:227-247 https://doi.org/10.1007/s11207-004-3747-2

Wiegelmann T, Thalmann JK, Solanki SK (2014) The magnetic field in the solar atmosphere. Astron Astrophys Rev 22:78 https://doi.org/10.1007/s00159-0140078-7

Wiegelmann T, Xia LD, Marsch E (2005) Links between magnetic fields and plasma flows in a coronal hole. 432(1):L1-L4 https://doi.org/10.1051/0004-63 61:200500029

Wiengarten T, Fichtner H, Kleimann J, Kissmann R (2015) Implementing turbulence transport in the cronos framework and application to the propagation of CMEs. Astrophys J 805(2):155 https://doi.org/10.1088/0004-63 7X/805/2/155

Wiengarten T, Kleimann J, Fichtner H, Cameron R, Jiang J, Kissmann R, Scherer K (2013) MHD Simulation of the inner-heliospheric magnetic field: innerheliospheric MHD simulations. J Geophys Res Space Phys 118(1):29-44 https://doi.org/10.1029/2012JA018089

Wiengarten T, Kleimann J, Fichtner H, Kühl P, Kopp A, Heber B, Kissmann R (2014) Cosmic ray transport in heliospheric magnetic structures. I. Modeling background solar wind using the cronos magnetohydrodynamic code. Astrophys J 788(1):80 https://doi.org/10.1088/0004-637X/788/1/80

Wijsen N, Aran A, Pomoell J, Poedts S (2019a) Modelling three-dimensional transport of solar energetic protons in a corotating interaction region generated with EUHFORIA. Astron Astrophys 622:A28 https://doi.org/10.1051/ 0004-6361/201833958

Wijsen N, Aran A, Pomoell J, Poedts S (2019b) Interplanetary spread of solar energetic protons near a high-speed solar wind stream. Astron Astrophys 624:A47 https://doi.org/10.1051/0004-6361/201935139

Wilcox JM, Howard R (1968) A large-scale pattern in the solar magnetic field. Sol Phys 5(4):564-574 https://doi.org/10.1007/BF00147021

Wild JP, Smerd SF, Weiss AA (1963) Solar bursts. Ann Rev Astron Astrophys 1(1): 291-366 https://doi.org/10.1146/annurev.aa.01.090163.001451

Wilson RM (1987) Geomagnetic response to magnetic clouds. Planetary Space Sci 35:329-335 https://doi.org/10.1016/0032-0633(87)90159-0

Wimmer-Schweingruber RF, Crooker NU, Balogh A, Bothmer V, Forsyth RJ, Gazis P, Gosling JT et al (2006) Understanding Interplanetary coronal mass ejection signatures: report of working group B. Space Sci Rev 123(1-3):177-216 https://doi.org/10.1007/s11214-006-9017-x

Wimmer-Schweingruber RF, von Steiger R, Paerli R (1997) Solar wind stream interfaces in corotating interaction regions: SWICS/Ulysses results. Jgr 102(A8):17407-17418 https://doi.org/10.1029/97JA00951

Wood BE, Howard RA (2009) An empirical reconstruction of the 2008 April 26 coronal mass ejection. Astrophys J. 702(2):901-910 https://doi.org/10.1088/ 0004-637X/702/2/901

Wood BE, Howard RA, Thernisien A, Plunkett SP, Socker DG (2009) Reconstructing the 3D morphology of the 17 May 2008 CME. Sol Phys 259:163-178 https:// doi.org/10.1007/s11207-009-9391-0

Wood BE, Wu C-C, Lepping RP, Nieves-Chinchilla T, Howard RA, Linton MG, Socker DG (2017) A STEREO survey of magnetic cloud coronal mass ejections observed at Earth in 2008-2012. Astrophys J Suppl 229(2):29 https://doi.org/1 $0.3847 / 1538-4365 / 229 / 2 / 29$

Wood BE, Wu C-C, Rouillard AP, Howard RA, Socker DG (2012) A coronal hole's effects on coronal mass ejection shock morphology in the inner heliosphere. Astrophys J 755(1):43 https://doi.org/10.1088/0004-637X/755/1/43

Wu C-C, Dryer M, Wu ST, Wood BE, Fry CD, Liou K, Plunkett S (2011) Global threedimensional simulation of the interplanetary evolution of the observed geoeffective coronal mass ejection during the epoch 1-4 August 2010: geoeffective CME during 1-4 August 2010. J Geophys Res Space Phys 116(A12):n/a https://doi.org/10.1029/2011JA016947

Wu C-C, Kan L, Lepping RP, Vourlidas A, Plunkett S, Socker D, Wu ST (2017) Observation of an extremely large-density heliospheric plasma sheet compressed by an interplanetary shock at 1 AU. Sol Phys 292(8):109 https:// doi.org/10.1007/s11207-017-1114-3

Wu C-C, Lepping RP (2002) Effect of solar wind velocity on magnetic cloudassociated magnetic storm intensity. J Geophys Res (Space Physics) 107:1346 https://doi.org/10.1029/2002JA009396 
Wu C-C, Liou K, Lepping RP, Hutting L, Plunkett S, Howard RA, Socker D (2016a) The first super geomagnetic storm of solar cycle 24: 'the St. Patrick's Day event (17 March 2015). Earth Planets Space 68:151 https://doi.org/10.1186/s4 0623-016-0525-y

Wu C-C, Liou K, Vourlidas A, Plunkett S, Murray D, Wu ST, Mewaldt RA (2016b) Global magnetohydrodynamic simulation of the 15 March 2013 coronal mass ejection event-interpretation of the 30-80 MeV proton flux: global simulation for 15 MARCH 2013 CME. J Geophys Res Space Phys 121(1):56-76 https://doi.org/10.1002/2015JA021051

Wu C-C, Liou K, Vourlidas A, Plunkett S, Murray D, Wu ST, Socker D, Wood BE, Hutting L, Howard RA (2016c) Numerical simulation of multiple CME-driven shocks in the month of 2011 September: CME-driven shocks in September 2011. J Geophys Res Space Phys 121(3):1839-1856 https://doi.org/10.1002/2 $015 J A 021843$

Wu ST, Zhou Y, Jiang C, Feng X, Wu C-C, Qiang H (2016d) A data-constrained three-dimensional magnetohydrodynamic simulation model for a coronal mass ejection initiation. J Geophys Res Space Phys 121(2):1009-1023 https:// doi.org/10.1002/2015JA021615

Xie H, Ofman L, Lawrence G (2004) Cone model for halo CMEs: application to space weather forecasting. J Geophys Res Space Phys 109(A3)

Xiong $\mathrm{M}$, Zheng $\mathrm{H}$, Wang S (2009) Magnetohydrodynamic simulation of the interaction between two interplanetary magnetic clouds and its consequent geoeffectiveness: 2. Oblique collision: MC-MC interaction and geoeffectiveness, 2. J Geophys Res Space Phys 114(A11):n/a https://doi.org/1 $0.1029 / 2009 J A 014079$

Xiong M, Zheng H, Wang Y, Wang S (2006) Magnetohydrodynamic simulation of the interaction between interplanetary strong shock and magnetic cloud and its consequent geoeffectiveness: 2. Oblique collision. J Geophys Res 111(A11):A11102 https://doi.org/10.1029/2006JA011901

Xu F, Borovsky JE (2015) A new four-plasma categorization scheme for the solar wind. J Geophys Res (Space Physics) 120(1):70-100 https://doi.org/10.1002/2 014JA020412

Xu M, Shen C, Wang Y, Luo B, Chi Y (2019) Importance of shock compression in enhancing ICME's geoeffectiveness. Astrophys J 884(2):L30 https://doi.org/1 $0.3847 / 2041-8213 / a b 4717$

Yalim MS, Pogorelov N, Yang L (2017) A data-driven MHD model of the global solar corona within Multi-Scale Fluid-Kinetic Simulation Suite (MS-FLUKSS). J Phys Conf Ser Webb 837:012015 https://doi.org/10.1088/1742-6596/837/1/ 012015

Yan XL, Wang JC, Pan GM, Kong DF, Xue ZK, Yang LH, Li QL, Feng XS (2018) Successive $X$-class flares and coronal mass ejections driven by shearing motion and sunspot rotation in active region NOAA 12673. Astrophys $J$ 856(1):79 https://doi.org/10.3847/1538-4357/aab153

Yang Y, Shen F (2019) Modeling the global distribution of solar wind parameters on the source surface using multiple observations and the artificial neural network technique. Sol Phys 294(8):111 https://doi.org/10.1007/s11207-01 9-1496-5

Yashiro S, Gopalswamy N (2009) Statistical relationship between solar flares and coronal mass ejections. 257:233-243 https://doi.org/10.1017/S174392130902 9342

Yashiro S, Gopalswamy N, Akiyama S, Michalek G, Howard RA (2005) Visibility of coronal mass ejections as a function of flare location and intensity. J Geophys Res (Space Physics) 110:A12S05 https://doi.org/10.1029/2005JA0111 51

Yashiro S, Gopalswamy N, Michalek G, Cyr OCS, Plunkett SP, Rich NB, Howard RA (2004) A catalog of white light coronal mass ejections observed by the SOHO spacecraft. J Geophys Res (Space Physics) 109:A07105 https://doi.org/1 0.1029/2003JA010282

Yermolaev YI, Lodkina IG, Nikolaeva NS, Yermolaev MY (2017) Dynamics of largescale solar-wind streams obtained by the double superposed epoch analysis: 2. Comparisons of CIRs vs. sheaths and MCs vs. ejecta. Sol Phys 292(12):193 https://doi.org/10.1007/s11207-017-1205-1

Yermolaev YI, Lodkina IG, Nikolaeva NS, Yermolaev MY, Riazantseva MO, Rakhmanova LS (2018) Statistic study of the geoeffectiveness of compression regions CIRs and sheaths. J Atmos Sol Terr Phys 180:52-59 https://doi.org/1 0.1016/j.jastp.2018.01.027

Yermolaev YI, Nikolaeva NS, Lodkina IG, Yermolaev MY (2009) Catalog of largescale solar wind phenomena during 1976-2000. Cosmic Res 47(2):81-94 https://doi.org/10.1134/S0010952509020014

Yiğit E, Knížová PK, Georgieva K, Ward W (2016) A Review of Vertical coupling in the atmosphere-ionosphere system: effects of waves, sudden stratospheric warmings, space weather, and of solar activity. J Atmos Sol Terr Phys SI Vertical Coupling 141:1-12 https://doi.org/10.1016/j.jastp.2016.02.011

Yurchyshyn V (2008) Relationship between EIT posteruption arcades, coronal mass ejections, the coronal neutral line, and magnetic clouds. Astrophys J Lett 675:L49 https://doi.org/10.1086/533413

Yurchyshyn V, Abramenko V, Tripathi D (2009) Rotation of white-light coronal mass ejection structures as inferred from LASCO coronagraph. Astrophys J 705:426-435 https://doi.org/10.1088/0004-637X/705/1/426

Zank GP, Rice WKM, Wu CC (2000) Particle acceleration and coronal mass ejection driven shocks: a theoretical model. J Geophys Res Space Phys 105(A11):25079-25095

Zelina P, Silvia D, Cohen CMS, Mewaldt RA (2017) Time Evolution of elemental ratios in solar energetic particle events. Astrophys J 835(1):71

Zhang J, Blanco-Cano X, Nitta N, Srivastava N, Mandrini CH (2018a) Editorial: Earth-affecting solar transients. Sol Phys 293:80 https://doi.org/10.1007/s112 07-018-1302-9

Zhang J, Blanco-Cano X, Nitta N, Srivastava N, Mandrini CH (eds) (2018b) Earthaffecting solar transients. 1st ed, 2019th edn. New York, Springer

Zhang J, Cheng X, Ding M-D (2012) Observation of an evolving magnetic flux rope before and during a solar eruption. Nat Commun 3:747 https://doi. org/10.1038/ncomms1753

Zhang J, Dere KP (2006) A Statistical study of main and residual accelerations of coronal mass ejections. Astrophys J 649:1100-1109 https://doi.org/10.1086/ 506903

Zhang J, Dere KP, Howard RA, Bothmer V (2003) Identification of solar sources of major geomagnetic storms between 1996 and 2000. Astrophys J 582:520533 https://doi.org/10.1086/344611

Zhang J, Dere KP, Howard RA, Kundu MR, White SM (2001) On the temporal relationship between coronal mass ejections and flares. Astrophys J 559(1): 452-462 https://doi.org/10.1086/322405

Zhang J, Dere KP, Howard RA, Vourlidas A (2004) A study of the kinematic evolution of coronal mass ejections. Astrophys J 604(1):420-432 https://doi. org/10.1086/381725

Zhang J, Richardson IG, Webb DF, Gopalswamy N, Huttunen E, Kasper JC, Nitta NV et al (2007) Solar and interplanetary sources of major geomagnetic storms ( Dst $\leq-100$ NT) during 1996-2005: sources of geomagnetic storms. J Geophys Res Space Phys 112(A10):n/a https://doi.org/10.1029/2007JA012321

Zhang M, Qin G, Rassoul H (2009) Propagation of solar energetic particles in three-dimensional interplanetary magnetic fields. Astrophys J 692(1):109-132 https://doi.org/10.1088/0004-637X/692/1/109

Zhao L, Zhang M, Rassoul HK (2016b) Double power laws in the event-integrated solar energetic particle spectrum. Astrophys J 821:62 https://doi.org/10.3847/ 0004-637X/821/1/62

Zhao L, Li G, Ebert RW, Dayeh MA, Desai MI, Mason GM, Wu Z, Chen Y (2016a) Modeling transport of energetic particles in corotating interaction regions: a case study. J Geophys Res (Space Physics) 121:77-92 https://doi.org/10.1 002/2015JA021762

Zhao L, Zhang M, Rassoul HK (2017) The effects of interplanetary transport in the event-integrated solar energetic particle spectra. Astrophys J 836(1):31

Zhao M-X, Le G-M, Chi Y-T (2018) Investigation of the possible source for the solar energetic particle event on 2017 September 10. Res Astron Astrophys 18(7):074

Zhao X, Dryer M (2014) Current status of CME/shock arrival time prediction. Space Weather 12:448-469 https://doi.org/10.1002/2014SW001060

Zhao XP, Plunkett SP, Liu W (2002) Determination of geometrical and kinematical properties of halo coronal mass ejections using the cone model: halo coronal mass ejections. J Geophys Res Space Phys 107(A8):SSH 13-1-SSH 139 https://doi.org/10.1029/2001JA009143

Zhou GP, Wang JX, Zhang J, Chen PF, Ji HS, Dere K (2006) Two successive coronal mass ejections driven by the kink and drainage instabilities of an eruptive prominence. Astrophys J 651:1238-1244 https://doi.org/10.1086/507977

Zhou Y, Feng X (2017) Numerical study of the propagation characteristics of coronal mass ejections in a structured ambient solar wind. J Geophys Res Space Phys 122(2):1451-1462 https://doi.org/10.1002/2016JA023053

Zhou Y, Feng X, Zhao X (2014) Using a 3-D MHD simulation to interpret propagation and evolution of a coronal mass ejection observed by multiple spacecraft: the 3 April 2010 event. J Geophys Res Space Phys 119(12):93219333 https://doi.org/10.1002/2014JA020347

Zhou YF, Feng XS (2013) MHD numerical study of the latitudinal deflection of coronal mass ejection: study of CME'S deflection propagation. J Geophys Res Space Phys 118(10):6007-6018 https://doi.org/10.1002/2013JA018976 
Zhou YF, Feng XS, Wu ST, Du D, Shen F, Xiang CQ (2012) Using a 3-D spherical plasmoid to interpret the Sun-to-Earth propagation of the 4 November 1997 coronal mass ejection event: 3-D MHD simulation of CME propagation. J Geophys Res Space Phys 117(A1) https://doi.org/10.1029/2010JA016380

Zhou Z, Cheng X, Zhang J, Wang Y, Dong W, Liu L, Zhuang B, Cui J (2019) Why do torus-unstable solar filaments experience failed eruptions? Astrophys J 877(2):L28 https://doi.org/10.3847/2041-8213/ab21cb

Zhu C, Qiu J, Liewer P, Vourlidas A, Spiegel M, Qiang H (2020) How does magnetic reconnection drive the early-stage evolution of coronal mass ejections? Astrophys J 893:141 https://doi.org/10.3847/1538-4357/ab838a

Zhuang B, Wang Y, Hu Y, Shen C, Liu R, Gou T, Zhang Q, Li X (2019) Numerical simulations on the deflection of coronal mass ejections in the interplanetary space. Astrophys J 876(1):73 https://doi.org/10.3847/1538-4357/ab139e

Zhukov AN, Auchère F (2004) On the nature of EIT waves, EUV dimmings and their link to CMEs. Astron Astrophys 427(2):705-716 https://doi.org/10.1051/ 0004-6361:20040351

Žic T, Vršnak B, Temmer M (2015) Heliospheric propagation of coronal mass ejections: drag-based model fitting. Astrophys J Suppl 218:32 https://doi. org/10.1088/0067-0049/218/2/32

Zuccarello FP, Aulanier G, Dudík J, Démoulin P, Schmieder B, Gilchrist SA (2017) Vortex and sink flows in eruptive flares as a model for coronal implosions. Astrophys J 837:115 https://doi.org/10.3847/1538-4357/aa6110

Zuccarello FP, Aulanier G, Gilchrist SA (2016) The apparent critical decay index at the onset of solar prominence eruptions. Astrophys J Lett 821:L23 https://doi. org/10.3847/2041-8205/821/2/L23

Zuccarello FP, Bemporad A, Jacobs C, Mierla M, Poedts S, Zuccarello F (2012) The role of streamers in the deflection of coronal mass ejections: comparison between STEREO three-dimensional reconstructions and numerical simulations. Astrophys J 744(1):66 https://doi.org/10.1088/0004-637X/744/1/66

Zurbuchen TH, Richardson IG (2006) In-situ solar wind and magnetic field signatures of interplanetary coronal mass ejections. Space Sci Rev 123:31-43 https://doi.org/10.1007/s11214-006-9010-4

\section{Publisher's Note}

Springer Nature remains neutral with regard to jurisdictional claims in published maps and institutional affiliations.

\section{Submit your manuscript to a SpringerOpen ${ }^{\circ}$ journal and benefit from:}

- Convenient online submission

- Rigorous peer review

- Open access: articles freely available online

High visibility within the field

- Retaining the copyright to your article

Submit your next manuscript at $\boldsymbol{\nabla}$ springeropen.com 